\title{
CONSTRUCTIBLE MOTIVIC FUNCTIONS AND MOTIVIC INTEGRATION
}

by

\author{
Raf Cluckers \& François Loeser
}

\section{Introduction}

1.1. - In this paper, intended to be the first in a series, we lay new general foundations for motivic integration and give answers to some important issues in the subject. Since its creation by Maxim Kontsevich [24], motivic integration developed quickly and has spread out in many directions. In a nutshell, in motivic integration, numbers are replaced by geometric objects, like virtual varieties, or motives. But, classicaly, not only numbers are defined using integrals, but also interesting classes of functions. The previous constructions of motivic integration were all quite geometric, and it was quite unclear how they could be generalized to handle integrals depending on parameters. The new approach we present here, based on cell decomposition, allows us to develop a very general theory of motivic integration taking parameters in account. More precisely, we define a natural class of functions - constructible motivic functions - which is stable under integration.

The basic idea underlying our approach is to construct more generally pushforward morphisms $f_{\text {! }}$ which are functorial - they satisfy $(f \circ g)_{!}=f_{!} \circ g_{!}$- so that performing motivic integration corresponds to taking the push-forward to the point. This strategy has many technical advantages. In essence, it allows to reduce the construction of $f_{\text {! }}$ to the case of closed immersions and projections, and in the latter case we can perform induction on the relative dimension, the basic case being that of relative dimension 1 , for which we can make use of the Cell Decomposition Theorem of Denef-Pas [30].

1.2. - Our main construction being inspired by analogy with integration along the Euler characteristic for constructible functions over the reals, let us first present a brief overview of this theory, for which we refer to [27], [32], and [37] for more details. We shall put some emphasis on formulation in terms of Grothendieck rings. Let us denote by $\mathrm{SA}_{\mathbb{R}}$ the category of real semialgebraic sets, that is, objects of $\mathrm{SA}_{\mathbb{R}}$ 
are semialgebraic sets and morphisms are semialgebraic maps. Since every real semialgebraic set admits a semialgebraic triangulation, the Euler characteristic of real semialgebraic sets may be defined as the unique $\mathbb{Z}$-valued additive invariant on the category of real semialgebraic sets which takes value one on closed simplexes. More precisely, let us define $K_{0}\left(\mathrm{SA}_{\mathbb{R}}\right)$, the Grothendieck ring of real semialgebraic sets, as the quotient of the free abelian group on symbols $[X]$, for $X$ real semialgebraic, by the relations $[X]=\left[X^{\prime}\right]$ if $X$ and $X^{\prime}$ are isomorphic, and $[X \cup Y]=[X]+[Y]-[X \cap Y]$, the product being induced by the cartesian product of semialgebraic sets. Then, existence of semialgebraic triangulations easily implies the following statement:

1.2.1. Proposition. - The Euler characteristic morphism $[X] \mapsto \operatorname{Eu}(X)$ induces a ring isomorphism

$$
K_{0}\left(\mathrm{SA}_{\mathbb{R}}\right) \simeq \mathbb{Z}
$$

A constructible function on a semialgebraic set $X$ is a function $\varphi: X \rightarrow \mathbb{Z}$ that can be written as a finite $\operatorname{sum} \varphi=\sum_{i \in I} m_{i} \mathbf{1}_{X_{i}}$ with $m_{i}$ in $\mathbb{Z}$,

$X_{i}$ semialgebraic subsets of $X$, and $\mathbf{1}_{X_{i}}$ the characteristic function of $X_{i}$. The set $\operatorname{Cons}(X)$ of constructible functions on $X$ is a ring. If $f: X \rightarrow Y$ is a morphism of semialgebraic sets, we have a natural pullback morphism $f^{*}: \operatorname{Cons}(Y) \rightarrow \operatorname{Cons}(X)$ given by $\varphi \mapsto \varphi \circ f$. Now let us explain how the construction of a push-forward morphism $f_{*}: \operatorname{Cons}(X) \rightarrow \operatorname{Cons}(Y)$ is related to integration with respect to Euler characteristic.

Let $\varphi=\sum_{i \in I} m_{i} \mathbf{1}_{X_{i}}$ be in $\operatorname{Cons}(X)$. One sets

$$
\int_{X} \varphi:=\sum_{i \in I} m_{i} \operatorname{Eu}\left(X_{i}\right) .
$$

It is quite easy to check that this quantity depends only on $\varphi$. Now if $f: X \rightarrow Y$ is a morphism, one checks that defining $f_{*}$ by

$$
f_{*}(\varphi)(y)=\int_{f^{-1}(y)} \varphi_{\mid f^{-1}(y)},
$$

indeed yields a morphism $f_{*}: \operatorname{Cons}(X) \rightarrow \operatorname{Cons}(Y)$, and that furthermore $(f \circ g)_{*}=$ $f_{*} \circ g_{*}$. For our purposes it will be more enlightening to express the preceding construction in terms of relative Grothendieck rings.

For $X$ a semialgebraic set, let us consider the category $\mathrm{SA}_{X}$ of semialgebraic sets over $X$. Hence objects of $\mathrm{SA}_{X}$ are morphisms $Y \rightarrow X$ in $\mathrm{SA}_{\mathbb{R}}$ and a morphism $(h: S \rightarrow X) \rightarrow\left(h^{\prime}: S^{\prime} \rightarrow X\right)$ in $\mathrm{SA}_{X}$ is a morphism $g: S \rightarrow S^{\prime}$ such that $h^{\prime} \circ g=h$. Out of $\mathrm{SA}_{X}$, one constructs a Grothendieck ring $K_{0}\left(\mathrm{SA}_{X}\right)$ similarly as before, and we have the following statement, which should be folklore, though we could not find it the literature.

1.2.2. Proposition. - Let $X$ be a semialgebraic set.

(1) The mapping $[h: S \rightarrow X] \mapsto h_{*}\left(\mathbf{1}_{S}\right)$ induces an isomorphism

$$
K_{0}\left(\mathrm{SA}_{X}\right) \simeq \operatorname{Cons}(X) .
$$


(2) Let $f: X \rightarrow Y$ be a morphism in $\mathrm{SA}_{\mathbb{R}}$. Under the above isomorphism $f_{*}: \operatorname{Cons}(X) \rightarrow \operatorname{Cons}(Y)$ corresponds to the morphism $K_{0}\left(\mathrm{SA}_{X}\right) \rightarrow K_{0}\left(\mathrm{SA}_{Y}\right)$ induced by composition with $f$.

1.3. - Let us now explain more about our framework. Fix a field $k$ of characteristic 0 . We want to integrate (functions defined on) subobjects of $k((t))^{m}$. For technical reasons it is wiser to consider more generally integration on subobjects of $k((t))^{m} \times k^{n} \times \mathbb{Z}^{r}$. This will allow considering parameters lying in the valued field, the residue field, and the value group. In fact, we shall restrict ourselves to considering a certain class of reasonably tame objects, that of definable subsets in a language $\mathcal{L}_{\mathrm{DP}}$. Typically these objects are defined by formulas involving usual symbols $0,1,+,-, \times$ for the $k((t))$ and $k$ variables, and $0,1,+,-, \leq$ for the $\mathbb{Z}$-variables, and also symbols ord for the valuation and $\overline{\mathrm{ac}}$ for the first non trivial coefficient of elements of $k((t))$, and the usual logical symbols (see 2.1 for more details). Furthermore we shall not only consider the set of points in $k((t))^{m} \times k^{n} \times \mathbb{Z}^{r}$ satisfying a given formula $\varphi$, but also look to the whole family of subsets of $K((t))^{m} \times K^{n} \times \mathbb{Z}^{r}$, for $K$ running over all fields containing $k$, of points that satisfy $\varphi$. This is what we call definable subassignments. Definable subassignments form a category and are our basic objects of study.

Let us fix such a definable subassignment $S$. Basically, constructible motivic functions on $S$ are built from

- classes $[Z]$ in a suitable Grothendieck ring of definable subassignments $Z$ of $S \times \mathbb{A}_{k}^{d}$ for some $d$;

- symbols $\mathbb{L}^{\alpha}$, where $\mathbb{L}$ stands for the class of the relative affine line over $S$ and $\alpha$ is some definable $\mathbb{Z}$-valued function on $S$;

- symbols $\alpha$ for $\alpha$ a definable $\mathbb{Z}$-valued function on $S$.

Constructible motivic functions on $S$ form a ring $\mathscr{C}(S)$. Any definable subassignment $C$ of $S$ has a characteristic function $\mathbf{1}_{C}$ in $\mathscr{C}(S)$.

1.4. - We explain now on an example how one can recover the motivic volume by considering the push-forward of constructible functions. We shall consider the points of the affine elliptic curve $x^{2}=y(y-1)(y-2)$ with nonnegative valuation, namely the definable subassignment $C$ of $\mathbb{A}_{k((t))}^{2}$ defined by the conditions

$$
x^{2}=y(y-1)(y-2), \quad \operatorname{ord}(x) \geq 0 \quad \text { and } \quad \operatorname{ord}(y) \geq 0 .
$$

Since the affine elliptic curve $E$ defined by $\xi^{2}=\eta(\eta-1)(\eta-2)$ in $\mathbb{A}_{k}^{2}$ is smooth, we know that the motivic volume $\mu(C)$ should be equal to $\frac{[E]}{\mathbb{L}}$, cf. [14]. Let us consider the projection $p: \mathbb{A}_{k(t))}^{2} \rightarrow \mathbb{A}_{k((t))}^{1}$ given by $(x, y) \mapsto y$. In our formalism $p_{!}\left(\left[\mathbf{1}_{C}\right]\right)$ is equal to a sum $A+B_{0}+B_{1}+B_{2}$ with

$$
\begin{gathered}
A=\left[\xi^{2}=\overline{\mathrm{ac}}(y)(\overline{\mathrm{ac}}(y)-1)(\overline{\mathrm{ac}}(y)-2)\right]\left[\mathbf{1}_{C(A)}\right] \\
B_{0}=\left[\xi^{2}=2 \overline{\mathrm{ac}}(y)\right]\left[\mathbf{1}_{C_{0}}\right] \mathbb{L}^{\operatorname{ord}(y) / 2} \\
B_{1}=\left[\xi^{2}=-\overline{\mathrm{ac}}(y-1)\right]\left[\mathbf{1}_{C_{1}}\right] \mathbb{L}^{\operatorname{ord}(y-1) / 2}
\end{gathered}
$$


and

$$
B_{2}=\left[\xi^{2}=2 \overline{\mathrm{ac}}(y-2)\right]\left[\mathbf{1}_{C_{2}}\right] \mathbb{L}^{\operatorname{ord}(y-2) / 2},
$$

with $C(A)=\{y \mid \operatorname{ord}(y)=\operatorname{ord}(y-1)=\operatorname{ord}(y-2)=0\}$ and $C_{i}=\{y \mid \operatorname{ord}(y-$ i) $>0$ and $\operatorname{ord}(y-i) \equiv 0 \bmod 2\}$. So $p_{!}\left(\left[\mathbf{1}_{C}\right]\right)$ looks already like a quite general constructible motivic function.

Let us show how one can recover the motivic volume of $\mu(C)$ by computing the integral of $p_{!}\left(\left[\mathbf{1}_{C}\right]\right)$ on $\mathbb{A}_{k((t)))}^{1}$. Let $\pi_{i}$ denote the projection of $\mathbb{A}_{k((t))}^{i}$ on the point. One computes $\pi_{1 !}(A)=\frac{[E]-3}{\mathbb{L}}$, while summing up the corresponding geometric series leads to that $\pi_{1 !}\left(B_{0}\right)=\pi_{1 !}\left(B_{1}\right)=\pi_{1 !}\left(B_{2}\right)=\mathbb{L}^{-1}$, so that finally $\pi_{1 !}\left(p_{!}\left(\left[1_{C}\right]\right)\right)=\frac{[E]}{\mathbb{L}}$. Hence the computation fits with the requirements $\pi_{1 !} \circ p_{!}=\left(\pi_{1} \circ p\right)$ ! $=\pi_{2 !}$ and $\pi_{2 !}\left(\left[1_{C}\right]\right)=\mu(C)$.

As we will see in the main construction of the push forward operator denoted with subscript !, in this example $p_{\text {! }}$ is calculated with "the line element" determined

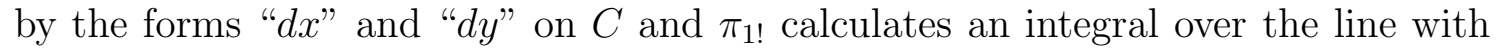
respect to the form " $d y$ " (see Theorem 10.1.1). In our context the line element is of course non-archimedean, see section 8.3 .

1.5. - Such a computation is maybe a bit surprising at first sight, since one could think that is not possible to recover the motive of an elliptic curve by projecting on to the line and computing the volume of the fibers, which consist of 0,1 or 2 points. The point is that our approach is not so naive and keeps track of the elliptic curve which remains encoded at the residue field level. Our main construction can be considered as a vast amplification of that example and one may understand that the main difficulty in the construction is proving that our construction of $f_{!}$is independent of the way we may decompose $f$ into a composition of morphisms.

In fact, we do not integrate functions in $\mathscr{C}(S)$, but rather their classes in a graded object $C(S)=\oplus_{d} C^{d}(S)$. The reason for that is that we have to take in account dimension considerations. For instance we could factor the identity morphism from the point to itself as the composition of an embedding in the line with the projection of the line on the point. But then a problem arises: certainly the point should be of measure 1 in itself, but as a subset of the line it should be of measure 0! To circumvent this difficulty, we filter $\mathscr{C}(S)$ by " $k((t))$-dimension of support". Typically, if $\varphi$ has " $k((t))$-dimension of support" equal to $d$, we denote by $[\varphi]$ its class in the graded piece $C^{d}(S)$. We call elements of $C(S)$ constructible motivic Functions (with capital F). One further difficulty is that arbitrary elements of $C(S)$ may not be integrable, that is, the corresponding integral could diverge. So we need to define at the same time the integral (or the push-forward) and the integrability condition. Also, as in the usual construction of Lebesgue integral, it is technically very useful to consider first only "positive constructible functions" on $S$. They form a semiring $\mathscr{C}_{+}(S)$ and we may consider the corresponding graded object $C_{+}(S)$.

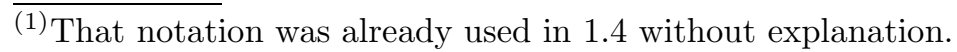


An important difference with the classical case, is that in general the canonical morphisms $\mathscr{C}_{+}(S) \rightarrow \mathscr{C}(S)$ and $C_{+}(S) \rightarrow C(S)$ are not injective.

The main achievement of the present paper is the following: we establish existence and uniqueness of a) a subgroup $\mathrm{I}_{S^{\prime}} C_{+}(S)$ of $C_{+}(S)$ consisting of $S^{\prime}$-integrable positive Functions on $S$, b) a push-forward morphism $f_{!}: \mathrm{I}_{S^{\prime}} C_{+}(S) \rightarrow C_{+}\left(S^{\prime}\right)$, under a certain system of natural axioms, for every morphism $f: S \rightarrow S^{\prime}$ of definable subassignments.

1.6. - Once the main result is proven, we can grasp its rewards. Firstly, it may be directly generalized to the relative setting of integrals with parameters. In particular we get that motivic integrals parametrized by a definable subassignment $S$ take their values in $\mathscr{C}_{+}(S)$ or in $\mathscr{C}(S)$. Also, our use of the quite abstract definable subassignments allows us to work at a level of generality that encompasses both "classical" motivic integration as developed in [14] and the "arithmetical" motivic integration of [15]. More precisely, we show that the present theory may be specialized both to "classical" motivic integration and "arithmetical" motivic integration, but with the bonus that no more completion process is needed. Indeed, there is a canonical forgetful morphism $\mathscr{C}$ (point) $\rightarrow K_{0}\left(\operatorname{Var}_{k}\right)_{\text {rat }}$, with $K_{0}\left(\operatorname{Var}_{k}\right)_{\text {rat }}$ the localization of the Grothendieck ring of varieties over $k$ with respect to $\mathbb{L}$ and $1-\mathbb{L}^{-n}, n \geq 1$, that sends the motivic volume of a definable object as defined here, to a representative of the "classical" motivic volume in $K_{0}\left(\operatorname{Var}_{k}\right)_{\text {rat }}$. So in the definable setting, "classical" motivic volume takes values in $K_{0}\left(\operatorname{Var}_{k}\right)_{\text {rat }}$ (and not in any completion of it). Such a result lies in the fact that in our machinery, the only infinite process that occurs is summation of geometric series in powers of $\mathbb{L}^{-1}$. A similar statement holds in the arithmetic case.

Another important feature is that no use at all is made of desingularization results. On the other side we rely very strongly on the Cell Decomposition Theorem of Denef-Pas. This makes in some sense things much worse in positive characteristic, since then desingularization is a reasonable conjecture while there is even no sensible guess of what cell decomposition could be in that case!

1.7. - Let us now describe briefly the content of the paper. Our basic objects of study are the various categories of definable subassignments in the Denef-Pas language that we review in section 2. An essential feature of these definable subassignments is that they admit a good dimension theory with respect to the valued field variables that we call $K$-dimension. This is established in section 3 . As a first step in constructing motivic integrals, we develop in section 4 a general machinery for summing over the integers. This is done in the framework of functions definable in the Presburger language. We prove a general rationality statement theorem 4.4.1 which we formulate in terms of a Mellin transformation. This allows to express punctual summability of a series in terms of polar loci of its Mellin transform and thus to define the sum of the series by evaluation of the Mellin transform at 1 . This construction is the main device that allows us to avoid any completion process in our integration theory, in contrast with previous approaches. In the following section 5 , 
we define constructible motivic functions and we extend the constructions and the results of the previous section to this framework. After the short section 6 which is devoted to the construction of motivic constructible Functions (as opposed to functions) and their relative variants, section 7 is devoted to cell decomposition, which is, as we already stressed, a basic tool in our approach. We need a definition of cells slightly more flexible than the one of Denef-Pas for which we give the appropriate Cell Decomposition Theorem à la Denef-Pas, and we also introduce bicells. We prove a fundamental structure result, Theorem [7.5.1, for definable functions with values in the valued field which may show interesting for its own right. Section 8 is devoted to introducing basic notions of differential calculus, like differential forms, volume forms and order of jacobian in the definable setting. In section 9, which appears to be technically quite involved, we construct motivic integrals in relative dimension 1 (with respect to the valued field variable). In particular we prove a fundamental change of variable formula in relative dimension 1 , whose proof uses Theorem 7.5.1, and which will be of essential use in the rest of the paper.

We are then able to state our main result, Theorem 10.1.1, in section 10, and section 11 is devoted to its proof. The idea of the proof is quite simple. By a graph construction one reduces to the case of definable injections and projections. Injections being quite easy to handle, let us consider projections. We already now how to integrate with respect to $\mathbb{Z}$-variables and also with respect to one valued field variable, integration with respect to residue field variables being essentially tautological. So to be able to deal with the general case, we need to prove various statements of Fubini type, that will allow us to interchange the order in which we perform integration with respect to various variables. The most difficult case is that of two valued field variables, that requires careful analysis of what happens on various types of bicells. Let us note that van den Dries encounters a similar difficulty in his construction of Euler characteristics in the o-minimal framework [20]. Once the main theorem is proved, we can derive the main properties and applications. In section [12, we prove a general change of variable formula and also the fundamental fact that a positive Function that is bounded above by an integrable Function is also integrable. We then develop the integration formalism for Functions in $C(X)$ - that is with no positivity assumption - in section 13. In section 14 we consider integrals with parameters and extend all previous resuts to this framework. As a side result, we prove the very general rationality theorem 14.4.1.

The last part of the paper is devoted to generalization to the global setting and to comparison results. In section [15, we consider integration on definable subsassignments of varieties. This is done by replacing functions by volume forms, as one can expect. More precisely, if $f$ is a morphism between global definable subassignments $S$ and $S^{\prime}$, we construct a morphism $f_{!}^{\text {top }}$ sending $f$-integrable volume forms on $S$ to volume forms on $S^{\prime}$, which corresponds to integrating Functions in top dimension in the affine case. This provides the right framework for a general Fubini Theorem for fiber integrals (Theorem 15.2.1). We then show in section 16] how our construction relates with the previous constructions of motivic integration. In the paper [8] we 
explain how it specializes to $p$-adic integration and we also give some applications to Ax-Kochen-Eršov Theorems for integrals depending on parameters. The main results of this paper have been anounced in the notes [6] and [7]. The present version of the paper does not differ from the original version except for very minor changes. Since our paper was originally put on the arxiv as math.AG/0410203, we have been able to extend our work to the exponential setting and to prove a general "Transfer principle" à la Ax-Kochen-Eršov in this context [9], [10]. Also Hrushovski and Kazhdan [25] developed a general theory of integration for valued fields based on Robinson's quantifier elimination for algebraically closed valued fields.

In writing the paper we tried our best keeping it accessible to a wide audience including algebraic geometers and model theorists. In particular, only basic familiarity with the first chapters of textbooks like those of Hartshorne [23] and Marker 28] is required. We also attempted to stay within the realm of geometry as much as possible. For example, we use, with the hope it would appeal to geometers, the terminology of "definable subassignments", introduced in [15], which is certainly familiar to logicians under other guises. By the foundational nature of the paper, many constructions and proofs are somewhat lengthy and technical, so we made an effort to make the main results and properties directly accessible and usable by the reader without having to digest all details. In particular, potential users might gain additional motivation by having a first look to sections [10, 12, 14 and 15] as early as possible. Also, one should stress that, for many applications, it is enough to consider integration in maximal dimension.

- - The present work would not exist without Jan Denef, whose insight and work, in particular concerning the ubiquity of cell decomposition, did have a strong influence on our approach. We also thank him warmly for his crucial encouragements when we started this project in February 2002. During the preparation of this work, we benefited from the support of many colleagues and friends. In particular, we would like to adress special thanks to Antoine Chambert-Loir, Clifton Cunningham, Lou van den Dries, Tom Hales and Udi Hrushovski for the interest they have shown in our work, and for comments and useful discussions that helped to improve the paper. The first author has been supported as a postdoctoral fellow by the Fund for Scientific Research - Flanders (Belgium) (F.W.O.) during the preparation of this paper. 


\section{Contents}

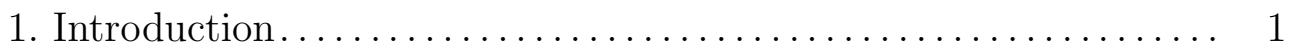

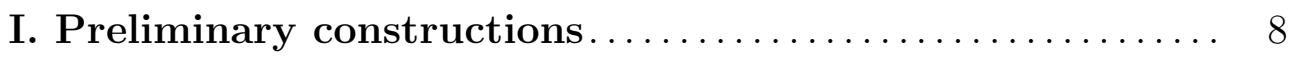

2. Definable subassignments....................... 8

3. Dimension theory for definable subassignments............. 13

4. Summation over Presburger sets.................... 18

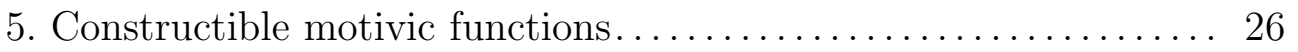

6. Constructible motivic Functions.................... 35

7. Cell decomposition................................ 36

8. Volume forms and Jacobians..................... 45

9. Integrals in dimension one........................ 49

II. Construction of the general motivic measure .......... 59

10. Statement of the main result ..................... 59

11. Proof of Theorem 10.1.1 . . . . . . . . . . . . . . . . . . 61

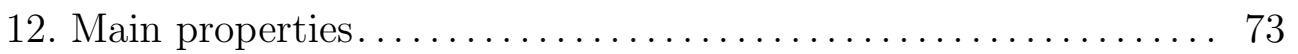

13. Integration of general constructible motivic Functions........ 75

14. Integrals with parameters .................... 78

III. Integration on varieties and comparison theorems .... . 82

15. Integration on varieties and Fubini Theorem............ 82

16. Comparison with the previous constructions of motivic integration 87

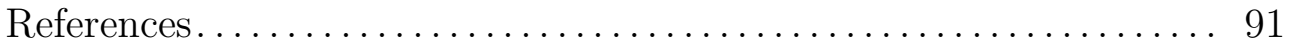

\section{I \\ PRELIMINARY CONSTRUCTIONS}

\section{Definable subassignments}

In this section, we extend the notion of definable subassignments, introduced in [15], to the context of $\mathcal{L}_{\mathrm{DP}}$-definable sets, with $\mathcal{L}_{\mathrm{DP}}$ a language of Denef-Pas.

2.1. Languages of Denef-Pas. - Let $K$ be a valued field, with valuation map ord : $K^{\times} \rightarrow \Gamma$ for some additive ordered group $\Gamma, R$ its valuation ring, $k$ the residue field. We denote by $x \mapsto \bar{x}$ the projection $R \rightarrow k$ modulo the maximal ideal $M$ of $R$. An angular component map (modulo $M$ ) on $K$ is a multiplicative map $\overline{\mathrm{ac}}: K^{\times} \rightarrow k^{\times}$ extended by putting $\overline{\mathrm{ac}}(0)=0$ and satisfying $\overline{\mathrm{ac}}(x)=\bar{x}$ for all $x$ with $\operatorname{ord}(x)=0$.

If $K=k((t))$ for some field $k$, there exists a natural valuation map $K^{\times} \rightarrow \mathbb{Z}$ and a natural angular component map sending $x=\sum_{i \geq \ell} a_{i} t^{i}$ in $K^{\times}$with $a_{i}$ in $k$ and $a_{\ell} \neq 0$ to $\ell$ and $a_{\ell}$, respectively. 
Fix an arbitrary expansion $\mathbf{L}_{\text {Ord }}$ of the language of ordered groups $(+,-, 0, \leq)$ and an arbitrary expansion $\mathbf{L}_{\text {Res }}$ of the language of rings $\mathbf{L}_{\text {Rings }}=(+,-, \cdot, 0,1)$. A language $\mathcal{L}_{\mathrm{DP}}$ of Denef-Pas is a three-sorted language of the form

$$
\left(\mathbf{L}_{\text {Val }}, \mathbf{L}_{\text {Res }}, \mathbf{L}_{\text {Ord }}, \text { ord, } \overline{\mathrm{ac}}\right) \text {, }
$$

with as sorts:

(i) a Val-sort for the valued field-sort,

(ii) a Res-sort for the residue field-sort, and

(iii) an Ord-sort for the value group-sort,

where the language $\mathbf{L}_{\mathrm{Val}}$ for the Val-sort is the language of rings $\mathbf{L}_{\text {Rings }}$, and the languages $\mathbf{L}_{\text {Res }}$ and $\mathbf{L}_{\text {Ord }}$ are used for the Res-sort and the Ord-sort, respectively. We only consider structures for $\mathcal{L}_{\mathrm{DP}}$ consisting of tuples $(K, k, \Gamma)$ where $K$ is a valued field with value group $\Gamma$, residue field $k$, a valuation map ord, and an angular component map $\overline{\mathrm{ac}}$, together with an interpretation of $\mathbf{L}_{\mathrm{Res}}$ and $\mathbf{L}_{\text {Ord }}$ in $k$ and $\Gamma$, respectively.

When $\mathbf{L}_{\text {Res }}$ is $\mathbf{L}_{\text {Rings }}$ and $\mathbf{L}_{\text {Ord }}$ is the Presburger language

$$
\mathbf{L}_{\mathrm{PR}}=\{+,-, 0,1, \leq\} \cup\left\{\equiv_{n} \mid n \in \mathbb{N}, n>1\right\},
$$

with $\equiv_{n}$ the equivalence relation modulo $n$ and 1 a constant symbol (with the natural interpretation if $\Gamma=\mathbb{Z}$ ), we write $\mathcal{L}_{\mathrm{DP}, \mathrm{P}}$ for $\mathcal{L}_{\mathrm{DP}}$.

As is standard for first order languages, $\mathcal{L}_{\mathrm{DP}}$-formulas are (meaningfully) built up from the $\mathcal{L}_{\mathrm{DP}}$-symbols together with variables, the logical connectives $\wedge$ (and), $\vee$ (or), $\neg$ (not), the quantifiers $\exists, \forall$, the equality symbol $=$, and parameter 2 .

Let us now recall the statement of the Denef-Pas Theorem on elimination of valued field quantifiers. Fix a language $\mathcal{L}_{\mathrm{DP}}$ of Denef-Pas. Denote by $H_{\overline{\mathrm{ac}}, 0}$ the $\mathcal{L}_{\mathrm{DP}}$-theory of the above described structures whose valued field is Henselian and whose residue field is of characteristic zero.

2.1.1. Theorem (Denef-Pas). - The theory $H_{\overline{\mathrm{ac}}, 0}$ admits elimination of quantifiers in the valued field sort. More precisely, every $\mathcal{L}_{\mathrm{DP}}$-formula $\phi(x, \xi, \alpha)$ (without parameters), with $x$ variables in the Val-sort, $\xi$ variables in the Res-sort and $\alpha$ vari-

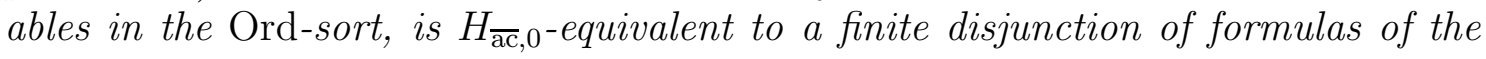
form

$$
\psi\left(\overline{\mathrm{ac}} f_{1}(x), \ldots, \overline{\mathrm{ac}} f_{k}(x), \xi\right) \wedge \vartheta\left(\operatorname{ord} f_{1}(x), \ldots, \text { ord } f_{k}(x), \alpha\right),
$$

with $\psi$ a $\mathbf{L}_{\text {Res }}$-formula, $\vartheta$ a $\mathbf{L}_{\text {Ord }}$-formula and $f_{1}, \ldots, f_{k}$ polynomials in $\mathbb{Z}[X]$.

Theorem 2.1.1 is not exactly expressed this way in [30]. The present statement can be found in [19] (3.5) and (3.7). We will mostly use the following corollary, which is standard in model theory.

(2) For first order languages, function symbols need to have a Cartesian product of sorts as domain, while the symbol ord has the valued field-sort minus the point zero as domain. Our use of the symbol ord with argument $x$ in a $\mathcal{L}_{\mathrm{DP}}$-formula is in fact an abbreviation for a function with domain the Val-sort which extends the valuation (the reader may choose the value of 0 ), conjoined with the condition $x \neq 0$. 
2.1.2. Corollary. - Let $(K, k, \Gamma)$ be a model of $H_{\overline{\mathrm{ac}}, 0}, S \subset K$ be a subring, $T_{S}$ be the diagram of $S$ in the language $\mathcal{L}_{\mathrm{DP}} \cup S$, that is, $T_{S}$ is the set of atomic $\mathcal{L}_{\mathrm{DP}} \cup S$ formulas and negations of atomic formulas $\varphi$ such that $S \models \varphi$, and $H_{S}$ be the union of $H_{\overline{\mathrm{ac}}, 0}$ and $T_{S}$. Then Theorem 2.1.1 holds with $H_{\overline{\mathrm{ac}}, 0}$ replaced by $H_{S}, \mathcal{L}_{\mathrm{DP}}$ replaced by $\mathcal{L}_{\mathrm{DP}} \cup S$, and $\mathbb{Z}[X]$ replaced by $S[X]$.

2.2. General subassignments. - Let $F: \mathscr{C} \rightarrow$ Sets be a functor from a category $\mathscr{C}$ to the category of sets. Any data, which associates to each object $C$ of $\mathscr{C}$ a subset $h(C)$ of $F(C)$, will be called a subassignment of $F$. The point in this definition is that $h$ is not assumed to be a subfunctor of $F$.

For $h$ and $h^{\prime}$ two subassignments of $F$, we shall denote by $h \cap h^{\prime}$ and $h \cup h^{\prime}$, the subassignments $C \mapsto h(C) \cap h^{\prime}(C)$ and $C \mapsto h(C) \cup h^{\prime}(C)$, respectively. Similarly, we denote by $h^{\prime} \backslash h$ the subassignment $C \mapsto h^{\prime}(C) \backslash h(C)$.

We also write $h^{\prime} \subset h$ if $h^{\prime}(C) \subset h(C)$ for every object $C$ of $\mathscr{C}$. In the case where $h^{\prime} \subset h$ are subassignments of $F$ we will also call $h^{\prime}$ a subassignment of $h$ (although $h$ itself need not to be a functor).

There is a trivial notion of a morphism between subassignments: for $h_{1}$ and $h_{2}$ subassignments of some functors $F_{1}, F_{2}: \mathscr{C} \rightarrow$ Sets, a morphism $f: h_{1} \rightarrow h_{2}$ is just the datum, for every object $C$ of $\mathscr{C}$, of a function $f(C)$ (or $f$ for short) from $h_{1}(C)$ to $h_{2}(C)$. If $h_{i}^{\prime}$ is a subassignment of $h_{i}, i=1,2$, one defines the subassignments $f\left(h_{1}^{\prime}\right)$ and $f^{-1}\left(h_{2}^{\prime}\right)$ in the obvious way. We can also define the Cartesian product $h_{1} \times h_{2}$ of $h_{1}$ and $h_{2}$ by $\left(h_{1} \times h_{2}\right)(C):=h_{1}(C) \times h_{2}(C)$ for every object $C$ of $\mathscr{C}$; it is a subassignment of the functor $F_{1} \times F_{2}$ which sends an object $C$ of $\mathscr{C}$ to $F_{1}(C) \times F_{2}(C)$. Similarly, one can perform other operations of set theory, for example:

The graph of a morphism $f: h_{1} \rightarrow h_{2}$ with $h_{i}$ a subassignment of $F_{i}$ is the subassignment of $F_{1} \times F_{2}$ sending an object $C$ of $\mathscr{C}$ to

$$
\left\{(x, y) \in h_{1}(C) \times h_{2}(C) \mid f(x)=y\right\} .
$$

If $h_{i}$ for $i=1,2,3$ are subassignments of $F_{i}: \mathscr{C} \rightarrow$ Sets and $f_{j}: h_{j} \rightarrow h_{3}$ morphisms for $j=1,2$, the fiber product $h_{1} \otimes_{h_{3}} h_{2}$ is the subassignment of $F_{1} \times F_{2}$ sending an object $C$ of $\mathscr{C}$ to

$$
\left\{(x, y) \in h_{1}(C) \times h_{2}(C) \mid f_{1}(x)=f_{2}(y)\right\} .
$$

2.3. Definable subassignments. - Let $k$ be a field. We denote by Field $_{k}$ the category of all fields containing $k$. More precisely, to avoid any set-theoretical issue, we shall fix a Grothendieck universe $\mathcal{U}$ containing $k$ and we define Field $_{k}$ as the small category of all fields in $\mathcal{U}$ containing $k$.

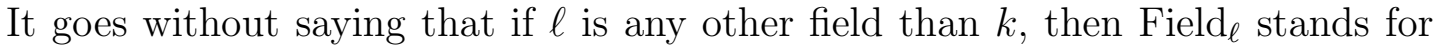
the category of all fields containing $\ell$. Although $k$ plays the role of standard base field throughout the paper, the definitions of section 2 make sense over any base field $\ell$ instead of $k$.

We consider $W:=\mathbb{A}_{k((t))}^{m} \times \mathbb{A}_{k}^{n} \times \mathbb{Z}^{r}, m, n, r \geq 0$. It defines a functor $h_{W}$ from the category Field $_{k}$ to the category of sets by setting $h_{W}(K)=K((t))^{m} \times K^{n} \times \mathbb{Z}^{r}$. We shall write $h[m, n, r]$ for $h_{W}$, where the base field $k$ is implicit in the notation 
$h[m, n, r]$; thus to avoid confusion, we only use this notation when the base field is clear. However, we will usually explicitly write $h_{\text {Spec } k}$ instead of $h[0,0,0]$; it is the functor which assigns to each $K$ in Field $k$ the one point set.

Fix a language $\mathcal{L}_{\mathrm{DP}}$ of Denef-Pas ${ }^{(3)}$. Any formula $\varphi$ in $\mathcal{L}_{\mathrm{DP}}$ with coefficients in $k((t))$ in the valued field sort and coefficients in $k$ in the residue field sort, with $m$ free variables in the valued field sort, $n$ in the residue field sort and $r$ in the value group sort, defines a subassignment $h_{\varphi}$ of $h[m, n, r]$ by assigning to $K$ in Field $_{k}$ the subset of $h[m, n, r](K)$ defined by $\varphi$, namely,

$$
h_{\varphi}(K)=\{x \in h[m, n, r](K) \mid(K, K((t)), \mathbb{Z}) \models \varphi(x)\} .
$$

We call $h_{\varphi}$ a definable subassignment of $h[m, n, r]$.

If the coefficients of the formula $\varphi$ in the valued field sort all lie in some subring $S$ of $k((t))$ and the coefficients in the residue field sort are still allowed to be in $k$, we call $h_{\varphi}$ a $\mathcal{L}_{\mathrm{DP}}(S)$-definable subassignment and we write $\mathcal{L}_{\mathrm{DP}}(S)$ to denote the language $\mathcal{L}_{\mathrm{DP}}$ with such coefficients.

We denote by $\emptyset$ the empty definable subassignment which sends each $K$ in Field $k$ to the empty set $\emptyset$. Here again, the base field $k$ is implicit in the notation $\emptyset$, and we only use it when the base field is clear.

More generally, if $\mathcal{X}$ is a variety, that is, a separated and reduced scheme of finite type, over $k((t))$ and $X$ is a variety over $k$, we consider $W^{\prime}:=\mathcal{X} \times X \times \mathbb{Z}^{r}$ and the functor $h_{W^{\prime}}$ from Field $k$ to the category of sets which to $K$ assigns $h_{W^{\prime}}(K)=$ $\mathcal{X}(K((t))) \times X(K) \times \mathbb{Z}^{r}$. We will define definable subassignments of $h_{W^{\prime}}$ by a glueing procedure. Assume first $X$ is affine and embedded as a closed subscheme in $\mathbb{A}_{k}^{n}$ and similarly for $\mathcal{X}$ in $\mathbb{A}_{k((t))}^{m}$. We shall say a subassignment of $h_{W^{\prime}}$ is a definable subassignment if it is a definable subassignment of $h[m, n, r]$. Clearly, this definition is independent of the choice of the embedding of $X$ and $\mathcal{X}$ in affine spaces.

In general, a subassignment $h$ of $h_{W^{\prime}}$ will be a definable subassignment if there exist finite covers $\left(X_{i}\right)_{i \in I}$ of $X$ and $\left(\mathcal{X}_{j}\right)_{j \in J}$ of $\mathcal{X}$ by affine open subschemes (defined over $k$ and $k((t))$ respectively; such covers always exist) and definable subassignments $h_{i j}$ of $h_{\mathcal{X}_{i} \times X_{j} \times \mathbb{Z}^{r}}$, for $i$ in $I$ and $j$ in $J$, such that $h=\cup_{i, j} h_{i j}$. If $\mathcal{X}$ as well as its cover $\left(\mathcal{X}_{j}\right)_{j}$ is defined over some subring $S$ of $k((t))$, and if the $h_{i j}$ are $\mathcal{L}_{\mathrm{DP}}(S)$-definable subassignments, we call $h$ a $\mathcal{L}_{\mathrm{DP}}(S)$-definable subassignment.

For $i=1,2$, let $h_{i}$ be a definable subassignment of $h_{W_{i}}$ with $W_{i}=\mathcal{X}_{i} \times X_{i} \times \mathbb{Z}^{r_{i}}$, $\mathcal{X}_{i}$ a variety over $k((t))$, and $X_{i}$ a variety over $k$. A definable morphism $f: h_{1} \rightarrow h_{2}$ is a morphism $h_{1} \rightarrow h_{2}$ (as in section 2.2) whose graph is a definable subassignment of $h_{W_{1} \times W_{2}}$. If moreover $h_{1}, h_{2}$, and the graph of $f$ are $\mathcal{L}_{\mathrm{DP}}(S)$-definable subassignments for some subring $S$ of $k((t))$, we call $f$ a $\mathcal{L}_{\mathrm{DP}}(S)$-definable morphism.

The set-theoretical operations defined above for general subassignments also work at the level of definable subassignments, for example, fiber products of definable subassignments are again definable subassignments.

(3) Except in Theorem 2.1.1, we always assume without writing that $(K((t)), K, \mathbb{Z})$ is a structure for $\mathcal{L}_{\mathrm{DP}}$ for all fields $K$ under consideration (usually $K$ runs over a category of the form Field ${ }_{k}$ ). Starting from section 5 the language will be $\mathcal{L}_{\mathrm{DP}, \mathrm{P}}$, which satisfies this condition. 
Sometimes we call a definable morphism a definable function, especially when the image is $h_{\mathbb{Z}^{r}}$ for some $r$.

2.4. - Using our fixed language $\mathcal{L}_{\mathrm{DP}}$ of Denef-Pas, we define the category of (affine) definable subassignments $\operatorname{Def}_{k}$ (also written $\operatorname{Def}_{k}\left(\mathcal{L}_{\mathrm{DP}}\right)$ ), as the category whose objects are pairs $(Z, h[m, n, r])$ with $Z$ a definable subassignment of $h[m, n, r]$, a morphism between $(Z, h[m, n, r])$ and $\left(Z^{\prime}, h\left[m^{\prime}, n^{\prime}, r^{\prime}\right]\right)$ being a definable morphism $Z \rightarrow Z^{\prime}$, that is, a morphism of subassignments whose graph is a definable subassignment of $h\left[m+m^{\prime}, n+n^{\prime}, r+r^{\prime}\right]$. Similarly one defines the category of (global) definable subassignments $\operatorname{GDef}_{k}$ (also written $\operatorname{GDef}_{k}\left(\mathcal{L}_{\mathrm{DP}}\right)$ ), as the category whose objects are pairs $\left(Z, h_{W}\right)$ with $Z$ a definable subassignment of $h_{W}$, where $W$ is of the form $\mathcal{X} \times X \times \mathbb{Z}^{r}$ with $\mathcal{X}$ a $k((t))$-variety and $X$ a $k$-variety, a morphism between $\left(Z, h_{W}\right)$ and $\left(Z^{\prime}, h_{W^{\prime}}\right)$ being a definable morphism $Z \rightarrow Z^{\prime}$.

More generally if $Z$ is in $\operatorname{Def}_{k}$, resp. GDef $k$, one considers the category $\operatorname{Def}_{Z}$, resp. GDef $Z$, of objects over $Z$, that is, objects are definable morphisms $Y \rightarrow Z$ in $\operatorname{Def}_{k}$, resp. GDef ${ }_{k}$, and a morphism between $Y \rightarrow Z$ and $Y^{\prime} \rightarrow Z$ is just a morphism $Y \rightarrow Y^{\prime}$ making the obvious diagram commute.

For every morphism $f: Z \rightarrow Z^{\prime}$ in $\operatorname{Def}_{k}$, composition with $f$ defines a functor $f_{!}: \operatorname{Def}_{Z} \rightarrow \operatorname{Def}_{Z^{\prime}}$. Also, fiber product defines a functor $f^{*}: \operatorname{Def}_{Z^{\prime}} \rightarrow \operatorname{Def}_{Z}$, namely, by sending $Y \rightarrow Z^{\prime}$ to $Y \otimes_{Z^{\prime}} Z \rightarrow Z$. We use similar notations when $f: Z \rightarrow Z^{\prime}$ is a morphism in $\operatorname{GDef}_{k}$.

Let $Y$ and $Y^{\prime}$ be in $\operatorname{Def}_{k}$ (resp. GDef ${ }_{k}$ ). We write $Y \times Y^{\prime}$ for the object $Y \otimes_{h_{\text {Spec }}} Y^{\prime}$ of $\operatorname{Def}_{k}\left(\operatorname{resp} . \operatorname{GDef}_{k}\right)$. We shall also write $Y[m, n, r]$ for $Y \times h[m, n, r]$. Nevertheless, the notation $h[m, n, r]$ will only be used for the definable subassignment defined above, and never for a product $h \times h[m, n, r]$ when $h$ itself is a definable subassignment.

Note that $h_{\text {Spec } k}$ and $\emptyset$ are respectivly the final and the initial objects of $\operatorname{Def}_{k}$ and GDef $_{k}$.

For a subring $S$ of $k((t))$, we define $\operatorname{Def}_{k}\left(\mathcal{L}_{\mathrm{DP}}(S)\right)$ as the subcategory of $\operatorname{Def}_{k}$ whose objects are pairs $(Z, h[m, n, r])$ with $Z$ a $\mathcal{L}_{\mathrm{DP}}(S)$-definable subassignment of $h[m, n, r]$, and whose morphisms are $\mathcal{L}_{\mathrm{DP}}(S)$-definable morphisms. Similarly we define $\operatorname{GDef}_{k}\left(\mathcal{L}_{\mathrm{DP}}(S)\right)$, $\operatorname{Def}_{Z}\left(\mathcal{L}_{\mathrm{DP}}(S)\right)$, and $\operatorname{GDef}_{Z}\left(\mathcal{L}_{\mathrm{DP}}(S)\right)$ for some $Z$ is in $\operatorname{Def}_{k}$ or in $\mathrm{GDef}_{k}$, respectively $(4)$.

2.5. Extension of scalars. - Let $W=\mathcal{X} \times X \times \mathbb{Z}^{r}$, with $\mathcal{X}$ a $k((t))$-variety and $X$ a $k$-variety, let $K$ be in Field $_{k}$, and let $W^{\prime}=\mathcal{X}^{\prime} \times X^{\prime} \times \mathbb{Z}^{r}$, with $\mathcal{X}^{\prime}=\mathcal{X} \otimes \operatorname{Spec} K((t))$ and $X^{\prime}=X \otimes \operatorname{Spec} K$. The extension of scalars functor sends $Z=\left(Z_{0}, h_{W}\right)$ in GDef $_{k}$ to $Z \otimes h_{\text {Spec } K}:=\left(Z_{0}^{\prime}, h_{W^{\prime}}\right)$ in GDef ${ }_{K}$, where $Z_{0}^{\prime}$ is the definable subassignment of $h_{W^{\prime}}$ which is given by the same $\mathcal{L}_{\mathrm{DP}}$-formulas as $Z_{0}$ on affine covers of $\mathcal{X}^{\prime}$ and $X^{\prime}$ which are defined over $k((t))$ and $k$, respectively. Using graphs, one defines similarly

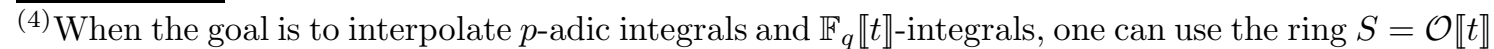
with $\mathcal{O}$ some ring of integers. This interpolation as well as a transfer result between $p$-adic and $\mathbb{F}_{q} \llbracket t \rrbracket$-integrals is announced in [8] 9] and detailed in [10]. 
the image of morphisms in GDef $_{k}$ under extension of scalars, getting a functor of extension of scalars GDef ${ }_{k} \rightarrow$ GDef $_{K}$.

2.6. Points on definable subassignments. - For $Z$ in $\operatorname{GDef}_{k}$, a point $x$ on $Z$ is by definition a tuple $x=\left(x_{0}, K\right)$ such that $x_{0}$ is in $Z(K)$ and $K$ is in Field ${ }_{k}$. For a point $x=\left(x_{0}, K\right)$ on $Z$ we write $k(x)=K$ and we call $k(x)$ the residue field of $x$.

Let $f: X \rightarrow Y$ be a morphism in $\operatorname{Def}_{k}$, with $X=\left(X_{0}, h[m, n, r]\right)$ and $Y=$ $\left(Y_{0}, h\left[m^{\prime}, n^{\prime}, r^{\prime}\right]\right)$. Let $\varphi(x, y)$ be the formula which describes the graph of $f$, where $x$ runs over $h[m, n, r]$ and $y$ runs over $h\left[m^{\prime}, n^{\prime}, r^{\prime}\right]$. For every point $y=\left(y_{0}, k(y)\right)$ of $Y$, we may consider its fibre $X_{y}$, which is the object in $\operatorname{Def}_{k(y)}$ defined by the formula $\varphi\left(x, y_{0}\right)$ (5). Taking fibers at $y$ gives rise to a functor $i_{y}^{*}: \operatorname{Def}_{Y} \rightarrow \operatorname{Def}_{k(y)}$.

Fibers of a morphism $f: X \rightarrow Y$ in GDef $_{k}$ are defined similarly via affine covers and we shall use similar notations as for morphisms in $\operatorname{Def}_{k}$.

2.7. T-subassignments. - Let $T$ be a theory given by sentences in $\mathcal{L}_{\text {DP }}$ with coefficients in $k$ and $k((t))$ (a sentence is a formula without any free variables). We denote by $\operatorname{Field}_{k}(T)$ the category of fields $F$ over $k$ such that $(F((t)), F, \mathbb{Z})$ is a model of $T$ and whose morphisms are field morphisms. Given a $k((t))$-variety $\mathcal{X}$, a $k$-variety $X$, and $W=\mathcal{X} \times X \times \mathbb{Z}^{r}$, we can restrict the functor $h_{W}$ as defined above to $\operatorname{Field}_{k}(T)$ and we also write $h_{W}$ to denote this functor. We can speak of definable $T$-subassignments of $h_{W}$ in exactly the same way as we did above for definable subassignments of $h_{W}$. A definable $T$-morphism between $T$-subassignments $Z$ and $Z^{\prime}$ is also defined accordingly.

We define the category $\operatorname{GDef}_{k}\left(\mathcal{L}_{\mathrm{DP}}, T\right)$ of definable $T$-subassignments as the category whose objects are pairs $\left(Z, h_{W}\right)$ with $Z$ a definable $T$-subassignment of $h_{W}$, where $W$ is of the form $W=\mathcal{X} \times X \times \mathbb{Z}^{r}$, and $T$-morphisms being definable $T$ morphisms. One defines similarly $\operatorname{Def}_{k}\left(\mathcal{L}_{\mathrm{DP}}, T\right)$.

For $S$ a subring of $k((t))$, if one restricts moreover the coefficients in the valued field sort to $S$, one defines the categories $\operatorname{Def}_{k}\left(\mathcal{L}_{\mathrm{DP}}(S), T\right)$ and $\operatorname{GDef}_{k}\left(\mathcal{L}_{\mathrm{DP}}(S), T\right)$ correspondingly.

\section{Dimension theory for definable subassignments}

3.1. - The Zariski closure of a definable subassignment $Z$ of $h_{\mathcal{X}}$ with $\mathcal{X}$ a variety over $k((t))$ is the intersection $W$ of all subvarieties $Y$ of $\mathcal{X}$ such that $Z \subset h_{Y}$. We define the dimension of $Z$ as $\operatorname{Kdim} Z:=\operatorname{dim} W$ if $W$ is not empty and as $-\infty$ if $W$ is empty. More generally, if $Z$ is a subassignment of $h_{W}$ with $W=\mathcal{X} \times X \times \mathbb{Z}^{r}, \mathcal{X}$ a variety over $k((t))$, and $X$ a variety over $k$, we define $\operatorname{Kdim} Z$ as the dimension of the image of $Z$ under the projection $h_{W} \rightarrow h_{\mathcal{X}}$. We shall establish basic properties of this dimension using work by van den Dries [18], by Denef and Pas [30], and by

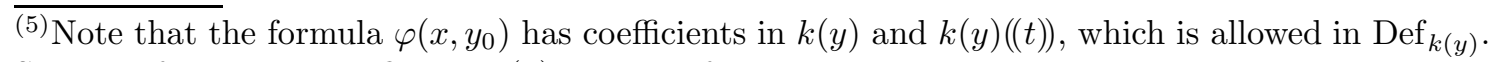
So, in $\operatorname{Def}_{k(y)}$, the base field is $k(y)$ instead of $k$. 
Denef and van den Dries 13. A similar dimension theory in a setting of first order languages with analytic functions has been developed by Çelikler in [3].

3.2. - Since $K((t))$ is a complete field for any field $K$, we can use the theory of $K((t))$-analytic manifolds as developed for instance in [1], thus using the $t$-adic topology on $K((t))$. By a $K((t))$-analytic manifold of dimension $n \geq 0$, we mean a separated topological space endowed with an analytic atlas of charts into $K((t))^{n}$. Note that we do not assume $K((t))$-analytic manifolds to have a countable basis for their topology. We shall consider the empty set as a $K((t))$-analytic manifold of dimension $-\infty$.

For any smooth equidimensional variety $\mathcal{X}$ over $k((t))$ and for any $K$ in Field $_{k}$ the set $\mathcal{X}(K((t)))$ has a natural structure of $K((t))$-analytic manifold. More generally, if $\mathcal{X}$ is a smooth equidimensional variety over $k((t)), X$ is a variety over $k, K$ is in Field $_{k}$, and $r$ is in $\mathbb{N}$, the set $h_{\mathcal{X} \times X \times \mathbb{Z}^{r}}(K)$ has a natural structure of $K((t))$-analytic manifold as product manifold of $\mathcal{X}(K((t)))$ and $X(K) \times \mathbb{Z}^{r}$ (the latter considered as a discrete set).

The following theorem asserts that definable subassignments are closely related to analytic manifolds; its proof is given below. We thank Çelikler for his help with the proof.

3.2.1. Theorem. - Let $Z$ be a nonempty definable subassignment of $h_{W}$ and $f$ : $Z \rightarrow h[1,0,0]$ a definable morphism, with $W=\mathcal{X} \times X \times \mathbb{Z}^{r}, \mathcal{X}$ a variety over $k((t))$, and $X$ a variety over $k$. Let $\left\{\mathcal{X}_{i}\right\}_{i}$ be a finite partition of $\mathcal{X}$ into smooth equidimensional varieties.

(i) There exists a finite partition of $Z$ into definable subassignments $Z_{j}$ such that, for each $K$ in Field $_{k}$ and each $j$, the set $Z_{j}(K)$ is a $K((t))$-analytic submanifold of $h_{\mathcal{X}_{i} \times X \times \mathbb{Z}^{r}}(K)$ for some $i$ only depending on $j$ and such that the restriction $f_{\mid Z_{j}}(K): Z_{j}(K) \rightarrow K((t))$ is $K((t))$-analytic.

(ii) If $Z_{i}$ is a partition as in (i), then $\mathrm{Kdim} Z$ equals

$$
\max _{j, K \in \text { Field }_{k},}\left(\operatorname{dim} Z_{j}(K)\right),
$$

where $\operatorname{dim} Z_{j}(K)$ denotes the dimension as a $K((t))$-analytic manifold.

(iii) There exists a definable subassignment $Z^{\prime}$ of $Z$ satisfying $\operatorname{Kdim}\left(Z \backslash Z^{\prime}\right)<$ $\operatorname{Kdim} Z$ and such that $Z^{\prime}(K)$ is a $K((t))$-analytic manifold and $f_{\mid Z^{\prime}}(K)$ : $Z^{\prime}(K) \rightarrow K((t))$ is $K((t))$-analytic for each $K$ in Field $_{k}$.

3.3. - Given a subassignment $Z$ of $h_{W}$ with $W$ as in Theorem 3.2.1 and $\mathcal{X} \rightarrow \mathcal{X}^{\prime}$ an embedding of $\mathcal{X}$ into a smooth equidimensional variety $\mathcal{X}^{\prime}$ over $k((t))$ and $K$ in Field $_{k}$, we endow $Z(K)$ with the induced topology coming from the manifold structure on $h_{\mathcal{X}^{\prime} \times X \times \mathbb{Z}^{r}}(K)$. This topology is independent of the embedding $\mathcal{X} \rightarrow$ $\mathcal{X}^{\prime}$. Many notions of general topology have a meaning in $\mathrm{GDef}_{k}$, for example, if $f: Z \rightarrow Y$ is a definable morphism in $\operatorname{GDef}_{k}$, we say $f$ is continuous if for each $K$ in Field $_{k}$ the map $f(K): Z(K) \rightarrow Y(K)$ is continuous. Similarly, for $Z \subset Y$ in GDef $_{k}$, one can construct definable subassignments $\operatorname{int}(Z)$ and $\operatorname{cl}(Z)$ such that for 
each $K$ in Field $_{k}$ the set $\operatorname{int}(Z)(K)$, resp. $\operatorname{cl} Z(K)$, is the interior, resp. the closure, of $Z(K)$ in $Y(K)$.

3.3.1. Theorem. - Let $Z$ and $Y$ be in GDef $_{k}$ and nonempty.

(i) If $f: Z \rightarrow Y$ is a definable morphism in $\operatorname{GDef}_{k}$, then $\operatorname{Kdim} Z \geq \operatorname{Kdim} f(Z)$. If $f$ is a definable isomorphism, then $\operatorname{Kdim} Z=\operatorname{Kdim} Y$.

(ii) The inequality

$$
\operatorname{Kdim}(Z \times Y) \leq \mathrm{K} \operatorname{dim} Z+\mathrm{K} \operatorname{dim} Y
$$

holds.

(iii) If $Z$ and $Y$ are definable subassignments of the same subassignment in GDef $_{k}$, one has

$$
\operatorname{Kdim}(Z \cup Y)=\max (\operatorname{Kdim} Z, \operatorname{Kdim} Y) .
$$

(iv) If $Z \subset Y$, let $\operatorname{cl}(Z)$ be the definable subassignment which is the closure of $Z$ in $Y$ as in 3.3. Then

$$
\operatorname{Kdim}(\operatorname{cl}(Z) \backslash Z)<\operatorname{Kdim} Z \text {. }
$$

(v) The integer $\mathrm{Kdim} Z$ is equal to the largest integer $d$ such that there exists a definable morphism $f: Z \rightarrow h[d, 0,0]$ such that $f(Z)$ has nonempty interior in $h[d, 0,0]$ for the topology of section [3.3, and with $f(Z)$ the image of $Z$ under $f$.

3.3.2. Example. - The inequality in (ii) of Theorem 3.3.1 can be strict: Suppose that -1 does not have a square root in $k$. Let $Z$, resp. $Y$, be a definable subassignment of $h[1,0,0]$ given by the formula $x=x \wedge \exists y \in h[1,0,0] y^{2}=-1$, resp. $x=x \wedge \forall y \in h[1,0,0] y^{2} \neq-1$, where $x$ runs over $h[1,0,0]$. Then the Zariski closure of both $Z$ and $Y$ is $\mathbb{A}_{k((t))}^{1}$, hence they both have dimension 1 , but the definable subassignment $Z \times Y$ is empty, hence its dimension is $-\infty$.

Proof of Theorem [3.3.1 - Let first $Z$ be a definable subassignment of $h[m, n, r]$ for some $m, n, r$, and $Y$ a definable subassignment of $h\left[m^{\prime}, n^{\prime}, r^{\prime}\right]$ for some $m^{\prime}, n^{\prime}, r^{\prime}$.

Let $\mathcal{L}$ be the language $\mathcal{L}_{\text {Val }}$ together with the following additional relation symbols:

(1) for each $\mathbf{L}_{\text {Ord }}$-formula $\varphi$ in $n$ free variables the $n$-ary relation symbol $R_{\varphi}$ interpreted as follows: $R_{\varphi}\left(a_{1}, \ldots, a_{n}\right)$ if and only if $\varphi\left(\operatorname{ord}\left(a_{1}\right), \ldots, \operatorname{ord}\left(a_{n}\right)\right)$ holds;

(2) for each $\mathbf{L}_{\text {Res }}$-formula $\varphi$ in $n$ free variables the $n$-ary relation symbol $R_{\varphi}$ interpreted as follows: $R_{\varphi}\left(a_{1}, \ldots, a_{n}\right)$ if and only if $\varphi\left(\overline{\operatorname{ac}}\left(a_{1}\right), \ldots, \overline{\operatorname{ac}}\left(a_{n}\right)\right)$ holds.

For each $K$ in Field ${ }_{k}$ let $\mathcal{L}_{K}$ be the language $\mathcal{L}$ with additional constant symbols for all elements of $K((t))$. Then, for each $K$ in Field $k$, it follows from Theorem 2.1.1 that the structure $\left(K((t)), \mathcal{L}_{K}\right)$ has elimination of quantifiers; moreover, $\left(K((t)), \mathcal{L}_{K}\right)$ satisfies the conditions of Proposition 2.15 of [18], with a topology as in 3.3. Thus, using the terminology of [18], there is a dimension function algdim - defined via the Zariski closure of definable sets - on $\mathcal{L}_{K}$-definable subsets of the structure $\left(K((t)), \mathcal{L}_{K}\right)$ for each $K$. 
We claim that

$$
\max _{K \in \operatorname{Field}_{k},(y, z) \in K^{n} \times \mathbb{Z}^{r}} \operatorname{algdim}\left(Z_{(y, z), K}\right)=\operatorname{Kdim} Z,
$$

with $Z_{(y, z), K}=Z(K) \cap\left(K((t))^{m} \times\{(y, z)\}\right)$, from which the theorem will follow.

The subassignment $Z$ is given by a formula $\varphi(x, y, z)$, where $x$ are the Valvariables, $y$ the Res-variables and $z$ the Ord-variables. By Denef-Pas quantifier elimination Theorem 2.1.1, we can write $\varphi$ as a disjunction over $j$ of formulas of the form

$$
\begin{aligned}
& \psi_{j}\left(z, \text { ord } f_{1 j}(x), \ldots, \text { ord } f_{r j}(x)\right) \wedge_{i} f_{i j}(x) \neq 0 \\
& \wedge \vartheta_{j}\left(y, \overline{\operatorname{ac}} g_{1 j}(x), \ldots, \overline{\operatorname{ac}} g_{s j}(x)\right) \wedge_{i} g_{i j}(x) \neq 0 \\
& \wedge h_{1 j}(x)=0 \wedge \ldots \wedge h_{t j}(x)=0
\end{aligned}
$$

with $f_{i j}, g_{i j}, h_{i j}$ polynomials over $k((t))$ - strictly speaking, these polynomials are defined over the constant symbols of $\mathcal{L}_{\text {Val }}$ - in the variables $x, \psi_{j} \mathbf{L}_{\text {Val }}$-formulas and $\vartheta_{j}$ $\mathbf{L}_{\text {Res }}$-formulas. Note that the first two lines of (3.3.2) determine open conditions. Let $V_{j}$ be the variety over $k((t))$ associated to the ideal $\left(h_{1 j}, \ldots, h_{t j}\right)$. Just by rewriting the disjunction (3.3.2), we may suppose that the $V_{j}$ are irreducible over $k((t))$. We prove (3.3.1) by induction on the maximum of the Zariski dimensions of the $V_{j}$. For each $j$ and each point $w=\left(y_{0}, z_{0}, K\right)$ in $h[0, n, r]$ let $U_{j w}$ be the definable subassignment in $\operatorname{Def}_{K}$ given by

$$
\begin{aligned}
& \psi_{j}\left(z_{0}, \operatorname{ord}\left(f_{1 j}(x)\right), \ldots, \operatorname{ord}\left(f_{r j}(x)\right)\right) \wedge_{i} f_{i j}(x) \neq 0 \\
& \wedge \vartheta_{j}\left(y_{0}, \overline{\mathrm{ac}}\left(g_{1 j}(x)\right), \ldots, \overline{\mathrm{ac}}\left(g_{s j}(x)\right)\right) \wedge_{i} g_{i j}(x) \neq 0 \\
& \wedge h_{1 j}(x)=0 \wedge \ldots \wedge h_{t j}(x)=0 \text {. }
\end{aligned}
$$

Let $j_{0}$ be such that $V_{j_{0}}$ has maximal Zariski dimension, say $\ell_{0}$, among the $V_{j}$. In the case where there exists $w=\left(y_{0}, z_{0}, K\right)$ such that the set $U_{j_{0} w}(K)$ has a Zariski closure over $K((t))$ of dimension equal to $\ell_{0}$, (3.3.1) follows. In the case where for all $w=\left(y_{0}, z_{0}, K\right)$ the set $U_{j_{0} w}(K)$ has a Zariski closure $V_{w}$ over $K((t))$ of dimension $<\ell_{0}$, then, since the field of definition of $V_{w}$ is contained in the algebraic closure of $k((t))$ intersected with $K((t))$, for each $w=\left(y_{0}, z_{0}, K\right)$, the set $U_{j_{0} w}(K)$ is contained in a Zariski closed set defined over $k((t))$ of dimension $<\ell_{0}$. Hence, by compactness, $V_{j_{0}}$ can be replaced by a $k((t))$-variety $V_{j_{0}}^{\prime}$ of dimension $<\ell_{0}$. This proves (3.3.1).

Theorem 3.3.1 now follows for $Z \subset h[m, n, r], Y \subset h\left[m^{\prime}, n^{\prime}, r^{\prime}\right]$ by (3.3.1) and by the equivalent properties of algdim stated in [18]. For example, to prove (i) we consider the definable morphism

$$
f^{\prime}:=\pi \times f: Z \rightarrow h[0, n, r] \times Y: x \longmapsto(\pi(x), f(x))
$$

with $\pi: Z \rightarrow h[0, n, r]$ the projection. We then compute

$$
\begin{aligned}
\operatorname{Kdim}(Z) & =\max _{K \in \text { Field }_{k}, x \in K^{n} \times \mathbb{Z}^{r}} \operatorname{algdim}\left(Z_{x, K}\right) \\
& \geq \max _{K \in \text { Field }_{k}, y \in K^{n+n^{\prime}} \times \mathbb{Z}^{r+r^{\prime}}}\left(\operatorname{algdim}\left(f^{\prime}(Z)_{y, K}\right)\right) \\
& =\operatorname{Kdim}^{\prime} f^{\prime}(Z) \\
& =\operatorname{Kdim} f(Z),
\end{aligned}
$$


with $f^{\prime}(Z)_{y, K}:=Y(K) \cap\left(K((t))^{m^{\prime}} \times\{y\}\right)$. Indeed, the inequality holds by the equivalent property for algdim for fixed $x, K$, and $y$ above $x$, and the last equality follows from the definition of Kdim .

These results extend to any definable subassignments $Z$ and $Y$ of functors of the form $h_{W}$ with $W=\mathcal{X} \times X \times \mathbb{Z}^{r}, \mathcal{X}$ a variety over $k((t)), X$ a variety over $k$, by using affine charts on $\mathcal{X}$ and $X$.

Proof of Theorem 3.2.1. - Theorem 3.2.1 for $Z \subset h[m, n, r]$ follows by the same proof as the proof of Lemma 3.12 of [13], the first part of the proof of Lemma 3.18 of [13], and the proof of Proposition 3.29 of [13]. For the convenience of the reader we give an outline of this argument and refer to [13] for the details.

We use the notation of the proof of Theorem 3.3.1. Let $Z \subset h[m, n, r], \varphi$, and $V_{j}$ be as in the proof of Theorem 3.3.1. If one takes a partition of each $V_{j}$ into smooth $k((t))$-subvarieties which are irreducible over $k((t))$, noting that the first two lines of (3.3.2) describe an open set, one can easily partition $Z$ into definable manifolds as in statement (i) by taking appropriate Boolean combinations (see Lemma 3.12 in [13] for details).

For the part of statement (i) about $f: Z \subset h[m, n, r] \rightarrow h[1,0,0]$ one uses induction on the dimension of $Z$ to obtain a finite partition of $Z$ such that the restriction of $f$ to each part is continuous (as in the proof of Proposition 3.29 in [13]), hence one may suppose that $f$ is continuous. Then one partitions the graph $\Gamma(f)$ of $f$ into manifolds as in (i) and one refines the partition in such a way that the tangent map of the projection $\pi: \Gamma(f) \rightarrow h[m, n, r]$ has constant rank on each part (as in the first part of the proof of Lemma 3.18 of [13]). It then follows that on each part of this partition the map $\pi$ is an analytic isomorphism between manifolds with analytic inverse $f$.

Statement (ii) for $Z \subset h[m, n, r]$ follows from Theorem 3.3.1 (iii) and (3.3.1) in its proof.

Statement (iii) for $Z \subset h[m, n, r]$ follows easily from (i) and Theorem 3.3.1, by taking the parts $Z_{j}$ of maximal dimension among the parts obtained in (i), and taking the union of $Z_{j} \backslash \cup_{i \neq j} \operatorname{cl}\left(Z_{i}\right)$ for $Z^{\prime}$.

Again, this extends to any definable subassignment $Z$ of $h_{W}$ with $W=\mathcal{X} \times X \times \mathbb{Z}^{r}$, $\mathcal{X}$ a variety over $k((t)), X$ a variety over $k$, by using affine charts on $\mathcal{X}$ and $X$.

3.4. Relative dimension. - Let $Z$ and $Y$ be in $\operatorname{GDef}_{k}$ and let $f: Z \rightarrow Y$ be a definable morphism. For every point $x$ on $Y$ let $Z_{x}$ be its fiber, as defined in section 2.6 .

For $i$ in $\mathbb{N} \cup\{-\infty\}$, we say $Z$ is of relative dimension $\leq i$ rel. $f$ if $\operatorname{Kdim} Z_{x} \leq i$ for every point $x$ in $Y$. We say $Z$ is equidimensional of relative dimension $i$ rel. $f$ if $\operatorname{Kdim} Z_{x}=i$ for every point $x$ in $Y$.

By Proposition 1.4 of [18] and by using similar arguments as the ones in the proof of Theorem 3.3.1, we deduce the following proposition:

3.4.1. Proposition. - Let $Z$ and $Y$ be in $\operatorname{GDef}_{k}$ and let $f: Z \rightarrow Y$ be a definable morphism. For every point $x$ on $Y$ let $Z_{x}$ be its fiber (as in section 2.6). The 
morphism $H: Y \rightarrow h_{\mathbb{Z}}$ which sends $x$ to $\operatorname{Kdim} Z_{x}$ if $Z_{x}$ is nonempty and to -1 otherwise is a definable morphism. For $i$ in $\mathbb{N}$ let $Y_{i}$ be the definable subassignment of $Y$ given by $H(x)=i$. Then, the definable subassignment $f^{-1}\left(Y_{i}\right)$ has dimension $i+\operatorname{Kdim} Y_{i}$.

The next proposition is a relative version of Theorem 3.2.1.

3.4.2. Proposition. - Let $\Lambda$ be in $\operatorname{Def}_{k}$. Let $Z \subset \Lambda[m, n, r]$ be a nonempty definable subassignment over $\Lambda$ for some $m, n, r$ such that $Z \rightarrow \Lambda$ is surjective. Let $f: Z \rightarrow h[1,0,0]$ be a definable morphism.

(i) There exists a finite partition of $Z$ into definable subassignments $Z_{j}$ such that, for each $\lambda$ in $\Lambda$, for each $K$ in Field $_{k(\lambda)}$, and each $j$, the fiber $i_{\lambda}^{*}\left(Z_{j}\right)(K)$ is a $K((t))$-analytic submanifold of $K((t))^{m} \times K^{n} \times \mathbb{Z}^{r}$ and such that the morphism

$$
f_{\mid i_{\lambda}^{*}\left(Z_{j}\right)}(K): i_{\lambda}^{*}\left(Z_{j}\right)(K) \longrightarrow K((t))
$$

is $K((t))$-analytic.

(ii) There exists a definable subassignment $Z^{\prime}$ of $Z$ satisfying

$$
\operatorname{Kdim}\left(i_{\lambda}^{*}(Z) \backslash i_{\lambda}^{*}\left(Z^{\prime}\right)\right)<\operatorname{Kdim} i_{\lambda}^{*}(Z)
$$

for each $\lambda$ in $\Lambda$ and such that, for each $K$ in Field $_{k(\lambda)}$, the fiber $i_{\lambda}^{*}\left(Z^{\prime}\right)(K)$ is a $K((t))$-analytic manifold on which $f_{\mid i_{\lambda}^{*}\left(Z^{\prime}\right)}(K)$ is $K((t))$-analytic.

Proof. - Apply Theorem 3.2.1 to $Z$, to $f$, and to the (Val coordinate functions of the) structure map $g: Z \rightarrow \Lambda$. Partition further into finitely many definable subassignments such that the restriction of $g$ to each of the parts has constant rank $d$ with respect to the Val-variables, and with a constant submatrix of the Jacobian matrix of $g$ of size $d$ having nonzero determinant. Now the proposition follows from the Implicit Function Theorem.

\section{Summation over Presburger sets}

4.1. Presbuger sets. - Let $G$ denote a $\mathbb{Z}$-group, that is, a group which is elementary equivalent to the integers $\mathbb{Z}$ in the Presburger language $\mathbf{L}_{\mathrm{PR}}$. We call $\left(G, \mathbf{L}_{\mathrm{PR}}\right)$ a Presburger structure. By a Presburger set, function, etc., we mean a $\mathbf{L}_{\mathrm{PR}}$-definable set, function. We recall that the theory $\operatorname{Th}\left(\mathbb{Z}, \mathbf{L}_{\mathrm{PR}}\right)$ has quantifier elimination in $\mathbf{L}_{\mathrm{PR}}$ and is decidable [31. Let $S$ be a Presburger set. We call a function

$$
f: X \subset S \times G^{m} \rightarrow G
$$

$S$-linear (or linear for short) if there is a definable function $\gamma$ from $S$ to $G$, and integers $0 \leq c_{i}<n_{i}$ and $a_{i}$, for $i=1, \ldots, m$, such that for every $x=\left(s, x_{1}, \ldots, x_{m}\right)$ in $X, x_{i}-c_{i} \equiv 0\left(\bmod n_{i}\right)$ and

$$
f(x)=\sum_{i=1}^{m} a_{i}\left(\frac{x_{i}-c_{i}}{n_{i}}\right)+\gamma(s) .
$$


We define similarly $S$-linear maps $g: X \rightarrow G^{n}$.

From now on in this section we shall assume that $G=\mathbb{Z}$.

4.2. Constructible Presburger functions. - We consider a formal symbol $\mathbb{L}$ and the ring

$$
\mathbb{A}:=\mathbb{Z}\left[\mathbb{L}, \mathbb{L}^{-1},\left(\frac{1}{1-\mathbb{L}^{-i}}\right)_{i>0}\right] .
$$

Note that for every real number $q>1$, there is a unique morphism of rings $\vartheta_{q}$ : $\mathbb{A} \rightarrow \mathbb{R}$ mapping $\mathbb{L}$ to $q$ and that, for $q$ transcendental, $\vartheta_{q}$ is injective. We define a partial ordering of $\mathbb{A}$ by setting $a \geq b$ if, for every real number $q>1, \vartheta_{q}(a) \geq \vartheta_{q}(b)$. We denote by $\mathbb{A}_{+}$the set $\{a \in \mathbb{A} \mid a \geq 0\}$. Note that $\mathbb{L}^{i}$, for $i$ in $\mathbb{Z}, \mathbb{L}^{i}-\mathbb{L}^{j}$, for $i>j$, and $\frac{1}{1-\mathbb{L}^{-i}}$, for $i>0$, all lie in $\mathbb{A}_{+}$, but, for instance, $\mathbb{L}-2$ does not. One has $a=b$ in $\mathbb{A}$ if and only if $\vartheta_{q}(a)=\vartheta_{q}(b)$ for all $q>1$. Indeed, considering a single transcendental $q>1$ is enough.

Now if $S$ is a definable subset of $\mathbb{Z}^{m}$ we define the ring $\mathcal{P}(S)$ of constructible Presburger functions on $S$ as the subring of the ring of functions $S \rightarrow \mathbb{A}$ generated by all constant functions into $\mathbb{A}$, all definable functions $S \rightarrow \mathbb{Z}$ and all functions of the form $\mathbb{L}^{\beta}$ with $\beta$ a $\mathbb{Z}$-valued definable function on $S$. We denote by $\mathcal{P}_{+}(S)$ the semiring of functions in $\mathcal{P}(S)$ with values in $\mathbb{A}_{+}$and write $f \geq g$ if $f-g$ is in $\mathcal{P}_{+}(S)$. This defines a partial ordering on $\mathcal{P}(S)$. When $S$ is one point we identify $\mathcal{P}(S)$ and $\mathbb{A}$.

4.3. Cell decomposition for Presburger sets. - In this subsection we recall the cell decomposition for Presburger sets as presented in [4]. Let $G$ denote a $\mathbb{Z}$ group. Fix a Presburger set $S$. We define Presburger cells parametrized by $S$, or Presburger $S$-cells.

4.3.1. Definition. - An $S$-cell of type (0) (also called a (0)-cell or cell for short) is a subset of $S \times G$ which is the graph of a $S$-linear function $S^{\prime} \rightarrow G$, with $S^{\prime}$ a definable subset of $S$. An $S$-cell of type (1) (also called (1)-cell or cell for short) is a subset $A$ of $S \times G$ of the form

$$
\left\{(s, x) \in S^{\prime} \times G \mid \alpha(s) \square_{1} x \square_{2} \beta(s), x \equiv c \quad(\bmod n)\right\},
$$

with $S^{\prime}$ a definable subset of $S, \alpha$ and $\beta S$-linear functions $S^{\prime} \rightarrow G, c$ and $n$ integers such that $0 \leq c<n$, and $\square_{i}$ either $\leq$ or no condition, and such that the cardinality of the fibers $A_{s}=\{x \in G \mid(s, x) \in A\}$ cannot be bounded uniformly for $s$ in $S^{\prime}$ by an integer.

Let us consider $i_{j}$ in $\{0,1\}$, for $j=1, \ldots, m$, and $x=\left(x_{1}, \ldots, x_{m}\right)$. A $\left(i_{1}, \ldots, i_{m}, 1\right)$-cell is a subset $A$ of $S \times G^{m+1}$ of the form

$$
A=\left\{(x, t) \in S \times G^{m+1} \mid x \in D, \alpha(x) \square_{1} t \square_{2} \beta(x), t \equiv c \quad(\bmod n)\right\},
$$

with $D=\pi_{m}(A)$ a $\left(i_{1}, \ldots, i_{m}\right)$-cell in $S \times G^{m}, \pi_{m}$ denoting the projection $S \times G^{m+1} \rightarrow$ $S \times G^{m}, \alpha, \beta: D \rightarrow G S$-linear functions, $\square_{i}$ either $\leq$ or no condition and integers $0 \leq c<n$ such that the cardinality of the fibers $A_{x}=\{t \in G \mid(x, t) \in A\}$ cannot 
be bounded uniformly for $x$ in $D$ by an integer.

A $\left(i_{1}, \ldots, i_{m}, 0\right)$-cell is a set of the form

$$
\left\{(x, t) \in S \times G^{m+1} \mid x \in D, \alpha(x)=t\right\},
$$

with $\alpha: D \rightarrow G$ a $S$-linear function and $D \subset S \times G^{m}$ a $\left(i_{1}, \ldots, i_{m}\right)$-cell.

A subset of $S \times G^{m}$ is called a $S$-cell if it is a $\left(i_{1}, \ldots, i_{m}\right)$-cell for some $i_{j}$ in $\{0,1\}$.

Now we can state the following:

\subsubsection{Theorem (Presburger Cell Decomposition [4])}

Let $S$ be a $\mathbf{L}_{\mathrm{PR}}$-definable set, let $X$ be a $\mathbf{L}_{\mathrm{PR}}$-definable subset of $S \times G^{m}$ and $f: X \rightarrow G$ a $\mathbf{L}_{\mathrm{PR}}$-definable map. Then there exists a finite partition $\mathcal{P}$ of $X$ into $S$-cells, such that the restriction $\left.f\right|_{A}: A \rightarrow G$ is $S$-linear for every cell $A$ in $\mathcal{P}$.

4.3.3. Remark. - Of course, one could assume $S$ is the one point set in the above statement, but it is more convenient to express it that way, in view of further generalizations.

4.4. The basic rationality result. - Let $S$ be a definable Presburger set. We consider the ring $\mathcal{P}(S) \llbracket T_{1}, \cdots, T_{r} \rrbracket$ of formal series with coefficients in the ring $\mathcal{P}(S)$. If $\alpha$ is a definable function on $S$ with values in $\mathbb{N}^{r}$, we write $T^{\alpha}$ for the series $\sum_{j \in \mathbb{N}^{r}} \mathbf{1}_{C_{j}} T^{j}$ in $\mathcal{P}(S) \llbracket T_{1}, \cdots, T_{r} \rrbracket$, where $\mathbf{1}_{C_{j}}$ is the characteristic function of the subset $C_{j}$ of $S$ defined by the formula $\alpha(x)=j$. We consider the subring $\mathcal{P}(S)\left\{T_{1}, \cdots, T_{r}\right\}$ of power series of the form $\sum_{i \in I} a_{i} T^{\alpha_{i}}$ with $I$ finite, $a_{i}$ in $\mathcal{P}(S)$, and $\alpha_{i}$ a definable function on $S$ with values in $\mathbb{N}^{r}$. In other words $\mathcal{P}(S)\left\{T_{1}, \cdots, T_{r}\right\}$ is the $\mathcal{P}(S)$-subalgebra of $\mathcal{P}(S) \llbracket T_{1}, \cdots, T_{r} \rrbracket$ generated by elements of the form $T^{\alpha}$ with $\alpha: S \rightarrow \mathbb{N}^{r}$ definable.

We denote by $\Gamma$ the multiplicative set of polynomials in $\mathcal{P}(S)\left[T_{1}, \cdots, T_{r}\right]$ generated by the polynomials $1-\mathbb{L}^{\alpha} T^{\beta}:=1-\mathbb{L}^{\alpha} \prod_{1<i<r} T_{i}^{\beta_{i}}$, for $\alpha$ in $\mathbb{Z}$ and $\beta=$ $\left(\beta_{1}, \cdots, \beta_{r}\right)$ in $\mathbb{N}^{r} \backslash\{(0, \cdots, 0)\}$. We denote by $\mathcal{P}(S)\left\{\bar{T}_{1}, \cdots, T_{r}\right\}_{\Gamma}$ the localisation of $\mathcal{P}(S)\left\{T_{1}, \cdots, T_{r}\right\}$ with respect to $\Gamma$. Since the polynomials $1-\mathbb{L}^{\alpha} T^{\beta}$ are invertible in $\mathcal{P}(S) \llbracket T_{1}, \cdots, T_{r} \rrbracket$, there exists a canonical morphism of rings

$$
\mathcal{P}(S)\left\{T_{1}, \cdots, T_{r}\right\}_{\Gamma} \longrightarrow \mathcal{P}(S) \llbracket T_{1}, \cdots, T_{r} \rrbracket,
$$

which is injective. We denote by $\mathcal{P}(S) \llbracket T_{1}, \cdots, T_{r} \rrbracket_{\Gamma}$, or by $\mathcal{P}(S) \llbracket T \rrbracket_{\Gamma}$ for short, the image of this morphism, which we identify with $\mathcal{P}(S)\left\{T_{1}, \cdots, T_{r}\right\}_{\Gamma}$.

We shall consider the $\mathcal{P}(S)$-module $\mathcal{P}(S) \llbracket T_{1}, T_{1}^{-1} \cdots, T_{r}, T_{r}^{-1} \rrbracket$, or $\mathcal{P}(S) \llbracket T, T^{-1} \rrbracket$ for short. Note that there is a natural product on $\mathcal{P}(S) \llbracket T, T^{-1} \rrbracket$, the Hadamard product, defined by

$$
f * g=\sum_{i \in \mathbb{Z}^{r}} f_{i} g_{i} T^{i}
$$

for $f=\sum_{i \in \mathbb{Z}^{r}} f_{i} T^{i}$ and $g=\sum_{i \in \mathbb{Z}^{r}} g_{i} T^{i}$, that endows $\mathcal{P}(S) \llbracket T, T^{-1} \rrbracket$ with a ring structure.

For $\varepsilon$ in $\{+1,-1\}^{r}$, we denote by $\varepsilon_{*}$ the $\mathcal{P}(S)$-module automorphism of $\mathcal{P}(S) \llbracket T, T^{-1} \rrbracket$ that sends $\sum_{i \in \mathbb{Z}^{r}} a_{i} T^{i}$ to $\sum_{i \in \mathbb{Z}^{r}} a_{i} T^{\varepsilon i}$ with $\varepsilon i=\left(\varepsilon_{1} i_{1}, \ldots, \varepsilon_{r} i_{r}\right)$. We 
denote by $\mathcal{P}(S) \llbracket T, T^{-1} \rrbracket_{\Gamma}$ the $\mathcal{P}(S)$-submodule of $\mathcal{P}(S) \llbracket T, T^{-1} \rrbracket$ generated by the submodules $\varepsilon_{*}\left(\mathcal{P}(S) \llbracket T \rrbracket_{\Gamma}\right)$ for all $\varepsilon$ in $\{+1,-1\}^{r}$.

For $\varphi$ in $\mathcal{P}\left(S \times \mathbb{Z}^{r}\right)$, and $i$ in $\mathbb{Z}^{r}$, we shall write $\varphi_{i}$ for the restriction of $\varphi$ to $S \times\{i\}$, viewed as an element of $\mathcal{P}(S)$, and consider the series

$$
\mathcal{M}(\varphi):=\sum_{i \in \mathbb{Z}^{r}} \varphi_{i} T^{i}
$$

in $\mathcal{P}(S) \llbracket T, T^{-1} \rrbracket$.

4.4.1. Theorem. - Let $S$ be a definable set. For every $\varphi$ in $\mathcal{P}\left(S \times \mathbb{Z}^{r}\right)$, the series $\mathcal{M}(\varphi)$ belongs to $\mathcal{P}(S) \llbracket T, T^{-1} \rrbracket_{\Gamma}$. Furthermore, the mapping $\varphi \mapsto \mathcal{M}(\varphi)$ induces $\mathcal{P}(S)$-algebra isomorphisms

$$
\mathcal{M}: \mathcal{P}\left(S \times \mathbb{Z}^{r}\right) \longrightarrow \mathcal{P}(S) \llbracket T, T^{-1} \rrbracket_{\Gamma}
$$

and

$$
\mathcal{M}: \mathcal{P}\left(S \times \mathbb{N}^{r}\right) \longrightarrow \mathcal{P}(S) \llbracket T \rrbracket_{\Gamma}
$$

the product on the power series rings being the Hadamard product.

4.4.2. Remark. - Note that $\mathcal{P}(S) \llbracket T, T^{-1} \rrbracket_{\Gamma}$ and $\mathcal{P}(S) \llbracket T \rrbracket_{\Gamma}$ are stable by Hadamard product since $\mathcal{M}$ is a bijection.

Proof. - The proof of the first statement is quite easy using the cell decomposition for Presburger sets recalled in Theorem 4.3.2 and quite similar statements (compare with Lemma 3.2 of [12]) may be found in the literature. Let us now prove that the $\mathcal{M}$ are isomorphisms. Take $\varphi$ in $\mathcal{P}\left(S \times \mathbb{Z}^{r}\right)$. We may first assume the support of $\varphi$ is contained in $S \times \mathbb{N}^{r}$. By the Cell Decomposition Theorem we may furthermore assume that the support of $\varphi$ is contained in a $S$-cell $A$ and that the restriction of $\varphi$ to $A$ is of the form $\prod_{1 \leq k \leq d} \alpha_{k} \mathbb{L}^{\beta}$ where $\alpha_{k}$ and $\beta$ are $S$-linear functions on $A$. Let us first consider the case $r=1$. When $A$ is a (0)-cell, there is nothing to prove. Assume now $A$ is a (1)-cell. Consider first the case where there is no condition $\square_{2}$ in (4.3.1). By Lemma 4.4.3, we can perform a direct computation of $\mathcal{M}(\varphi)$ (which essentially amounts to summing (derivatives) of geometric series of monomials in $\mathbb{L}$ and $T_{1}$ along an infinite arithmetic progression) which yields that $\mathcal{M}(\varphi)$ is a finite sum of terms of the form $\psi(s) \frac{T_{1}^{\gamma(s)}}{\left(1-\mathbb{L}^{a} T_{1}^{b}\right)^{c}}$, with $\psi(s)$ in $\mathcal{P}(S), \gamma: S \rightarrow \mathbb{N} S$-linear, $a$ in $\mathbb{Z}, b>0$ and $c$ in $\mathbb{N}$.

4.4.3. Lemma. - Let $R$ be a ring and let $P$ be a degree d polynomial in $R[X]$. The equality

$$
\sum_{n \geq a} P(n) T^{n}=\sum_{i=0}^{d} \frac{\left[\Delta^{i} P(a)\right] T^{a+i}}{(1-T)^{i+1}}
$$

holds in $R \llbracket T \rrbracket$ for all a in $\mathbb{N}$. Here $\Delta^{i}$ is the $i$-th iterate of the difference operator $P \mapsto P(X+1)-P(X)$ with the convention $\Delta^{0} P=P$. 
When there is a condition $\square_{2}$ in (4.3.1), we may express $\mathcal{M}(\varphi)$ as the difference of two series of the preceding type.

Consider now the case $r=2$. Let us first sum with respect to the variable $T_{2}$ in the series $\mathcal{M}(\varphi)$. By what we know about the case $r=1$, relatively to $S \times \mathbb{N}$, we get that $\mathcal{M}(\varphi)$ is a finite sum of terms of the form

$$
\sum_{i \in \mathbb{N}} \psi(s, i) T_{1}^{i} \frac{T_{2}^{\gamma(s, i)}}{\left(1-\mathbb{L}^{a} T_{2}^{b}\right)^{c}},
$$

with $\psi(s, i)$ in $\mathcal{P}(S \times \mathbb{N}), \gamma: S \times \mathbb{N} \rightarrow \mathbb{N}$ definable, $a$ in $\mathbb{Z}, b>0$ and $c$ in $\mathbb{N}$. So we just need summing up series of the type

$$
\sum_{i \in \mathbb{N}} \psi(s, i) T_{1}^{i} T_{2}^{\gamma(s, i)}
$$

which can be done exactly in the same way as the case $r=1$, except that instead of dealing with geometric series in monomials of $\mathbb{L}$ and $T_{1}$, we have now to deal with geometric series in monomials of $\mathbb{L}, T_{1}$ and $T_{2}$ which will have the effect of producing denominators of the form $1-\mathbb{L}^{a} T_{1}^{b} T_{2}^{c}$, with $a$ in $\mathbb{Z}$, and $b$ and $c$ strictly positive integers.

The case where $\varphi$ belongs to $\mathcal{P}\left(S \times \mathbb{Z}^{r}\right)$ for general $r$ is completely similar. Next we show that every element $m$ in $\mathcal{P}(S) \llbracket T, T^{-1} \rrbracket_{\Gamma}$ is of the form $\mathcal{M}(\varphi)$, by using Theorem-Definition 4.5.1. Note that this part of the theorem is not used in the proof of Theorem-Definition 4.5.1. We may assume $m$ is in $\mathcal{P}(S) \llbracket T \rrbracket_{\Gamma}$, and furthermore, by linearity, that it is of the form

$$
\frac{T^{\alpha}}{\prod_{i=1}^{\ell}\left(1-\mathbb{L}^{a_{i}} T^{b_{i}}\right)}
$$

with $\alpha: S \rightarrow \mathbb{N}^{r}$ definable, $a_{i}$ in $\mathbb{Z}$, and $b_{i}$ in $\mathbb{N}^{r} \backslash\{(0, \ldots, 0)\}$. The case of general $\alpha$ following easily from the case $\alpha=0$, we may assume $\alpha=0$. Let $H$ be the definable subassignment of $S \times \mathbb{N}^{r+\ell}$ given by the condition

$$
(s, c, d) \in S \times \mathbb{N}^{r+\ell} \wedge \sum_{i=1}^{\ell} d_{i} \cdot b_{i}=c .
$$

Note that for each $(s, c)$, the set of $d$ 's in $\mathbb{N}^{\ell}$ satisfying this condition is finite. Let $\beta: H \rightarrow \mathbb{Z}$ be the definable morphism $(s, c, d) \mapsto \sum_{i=1}^{\ell} d_{i} a_{i}$. Let $\psi$ be $\mathbb{L}^{\beta}$ in $\mathcal{P}\left(S \times \mathbb{N}^{r+\ell}\right)$. By the finiteness of the fibers, $\psi$ is $S \times \mathbb{N}^{r}$-summable in the sense of 4.5. If one sets $\varphi$ to be $\mu_{S \times \mathbb{N}^{r}}(\psi)$, as given by Theorem-Definition 4.5.1, then, by construction, $\mathcal{M}(\varphi)=m$, which is what we had to prove.

4.5. Summation of constructible Presburger functions. - Recall the notion of summable families in $\mathbb{R}$ or $\mathbb{C}$, cf. [2] VII.16. In particular, a family $\left(z_{i}\right)_{i \in I}$ of complex numbers is summable if and only if the family $\left(\left|z_{i}\right|\right)_{i \in I}$ is summable in $\mathbb{R}$. We shall say a family $\left(a_{i}\right)_{i \in I}$ in $\mathbb{A}$ is summable if, for every $q>1$, the family $\left(\vartheta_{q}\left(a_{i}\right)\right)_{i \in I}$ is summable in $\mathbb{R}$. We shall say a function $\varphi$ in $\mathcal{P}\left(S \times \mathbb{Z}^{r}\right)$ is $S$-integrable if, for 
every $s$ in $S$, the family $(\varphi(s, i))_{i \in \mathbb{Z}^{r}}$ is summable. We shall denote by $\operatorname{I}_{S} \mathcal{P}\left(S \times \mathbb{Z}^{r}\right)$ the $\mathcal{P}(S)$-module of $S$-integrable functions.

4.5.1. Theorem-Definition. - For each $\varphi$ in $\mathrm{I}_{S} \mathcal{P}\left(S \times \mathbb{Z}^{r}\right)$ there exists a unique function $\mu_{S}(\varphi)$ in $\mathcal{P}(S)$ such that for all $q>1$ and all $s$ in $S$

$$
\vartheta_{q}\left(\mu_{S}(\varphi)(s)\right)=\sum_{i \in \mathbb{Z}^{r}} \vartheta_{q}(\varphi(s, i))
$$

Moreover, the mapping $\varphi \mapsto \mu_{S}(\varphi)$ yields a morphism of $\mathcal{P}(S)$-modules

$$
\mu_{S}: \mathrm{I}_{S} \mathcal{P}\left(S \times \mathbb{Z}^{r}\right) \longrightarrow \mathcal{P}(S) .
$$

Proof. - By induction and Fubini's Theorem, it is enough to consider the case when $r=1$. Using Theorem 4.3.2 as in the proof of Theorem 4.4.1, we may assume that $\varphi$ is of the form

$$
\varphi(s, i)= \begin{cases}\mathbb{L}^{\frac{a(i-c)}{n}}\left(\frac{i-c}{n}\right)^{b} h(s) & \text { if } i \text { belongs to } I(s) \\ 0 & \text { otherwise }\end{cases}
$$

where $a$ lies in $\mathbb{Z}, b$ in $\mathbb{N}, s$ in $S, h$ in $\mathcal{P}(S)$, and

$$
I(s)=\left\{i \in \mathbb{Z} \mid \alpha(s) \square_{1} i \square_{2} \beta(s), i \equiv c \bmod n\right\},
$$

with $0 \leq c<n$ integers, $\alpha, \beta: S \rightarrow \mathbb{Z}$ definable functions, $\square_{i}$ either $<$ or no condition, and that the sum

$$
\sum_{i \in I(s)} q^{\frac{a(i-c)}{n}}\left(\frac{i-c}{n}\right)^{b} h(s)
$$

is summable for all $s$ in $S$ and $q>1$. Now the theorem follows from Lemma 4.4.3 and Lemma 4.5.2, which is a refinement of the claim in the proof of Lemma 3.2 of [12].

4.5.2. Lemma. - Let $b$ and $0 \leq c<n$ be integers. There exist Presburger functions $\gamma_{\ell j}: \mathbb{Z} \rightarrow \mathbb{Z}$ for $\ell$ in a finite set $L$ and $j=0, \ldots, b$, such that

$$
\sum_{\substack{0 \leq i \leq a \\ i \equiv c \bmod n}} i^{b}=\sum_{\ell \in L} \prod_{j=0, \ldots, b} \gamma_{\ell j}(a)
$$

for each $a \geq 0$.

4.5.3. Characterization of $\mathrm{I}_{S} \mathcal{P}$ in terms of power series. - We denote by $\mathcal{P}(S)\{\{T\}\}$ the subring of $\mathcal{P}(S) \llbracket T \rrbracket_{\Gamma}$ consisting of series with coefficients in $\mathcal{P}(S)$ such that, for every $s$ in $S$, at most a finite number of coefficients have non zero value at $s$. For instance, for $\gamma: S \rightarrow \mathbb{N}$, the series $\frac{1-T^{\gamma}}{1-T}$ belongs to $\mathcal{P}(S)\{\{T\}\}$, say if $r=1$. Let $\Sigma$ be the multiplicative set generated by the polynomials $1-\mathbb{L}^{\alpha} T^{\beta}:=1-\mathbb{L}^{\alpha} \prod_{1 \leq i \leq r} T_{i}^{\beta_{i}}$, for $\alpha$ in $\mathbb{Z} \backslash \mathbb{N}$ and $\beta=\left(\beta_{1}, \cdots, \beta_{r}\right)$ in $\mathbb{N}^{r} \backslash\{(0, \ldots, 0)\}$. We denote by $\mathcal{P}(S) \llbracket T \rrbracket_{\Sigma}$ the subring of $\mathcal{P}(S) \llbracket T \rrbracket_{\Gamma}$ (with the product being the usual product in power series rings) whose elements are of 
the form $\frac{P}{Q}$ with $P$ in $\mathcal{P}(S)\{\{T\}\}$ and $Q$ in $\Sigma$. The ring $\mathcal{P}(S) \llbracket T \rrbracket_{\Sigma}$ captures the summable series among the series in $\mathcal{P}(S) \llbracket T \rrbracket_{\Gamma}$, as is shown by Theorem 4.5.4. We shall denote by $\mathcal{P}(S) \llbracket T, T^{-1} \rrbracket_{\Sigma}$ the $\mathcal{P}(S)$-submodule of $\mathcal{P}(S) \llbracket T, T^{-1} \rrbracket_{\Gamma}$ generated by the submodules $\varepsilon_{*}\left(\mathcal{P}(S) \llbracket T \rrbracket_{\Sigma}\right)$ for all $\varepsilon$ in $\{+1,-1\}^{r}$.

4.5.4. Theorem. - Let $S$ be a definable set. The transformation $\mathcal{M}$ induces isomorphisms of $\mathcal{P}(S)$-modules

$$
\mathcal{M}: \mathrm{I}_{S} \mathcal{P}\left(S \times \mathbb{Z}^{r}\right) \longrightarrow \mathcal{P}(S) \llbracket T, T^{-1} \rrbracket_{\Sigma}
$$

and

$$
\mathcal{M}: \mathrm{I}_{S} \mathcal{P}\left(S \times \mathbb{N}^{r}\right) \longrightarrow \mathcal{P}(S) \llbracket T \rrbracket_{\Sigma}
$$

Proof. - Let $\varphi$ be in $\mathcal{P}\left(S \times \mathbb{Z}^{r}\right)$. We want to prove that $\varphi$ is $S$-integrable if and only if $\mathcal{M}(\varphi)$ lies in $\mathcal{P}(S) \llbracket T, T^{-1} \rrbracket_{\Sigma}$. We may assume the support of $\varphi$ is contained in $S \times \mathbb{N}^{r}$, so that $\mathcal{M}(\varphi)$ belongs to $\mathcal{P}(S) \llbracket T \rrbracket_{\Gamma}$. It is quite clear that if $\mathcal{M}(\varphi)$ belongs to $\mathcal{P}(S) \llbracket T \rrbracket_{\Sigma}$, then $\varphi$ is $S$-integrable. Assume now $\mathcal{M}(\varphi)$ is not in $\mathcal{P}(S) \llbracket T \rrbracket_{\Sigma}$. Then, there exists $s_{0}$ in $S$ and $q>1$ such that, extending $\vartheta_{q}$ coefficientwise to series,

$$
\vartheta_{q}(\mathcal{M}(\varphi))_{\mid s=s_{0}}=\frac{P_{s_{0}}\left(T_{1}, \ldots, T_{r}\right)}{Q_{s_{0}}\left(T_{1}, \ldots, T_{r}\right)}
$$

with $P_{s_{0}}$ and $Q_{s_{0}}$ in $\mathbb{R}\left[T_{1}, \ldots, T_{r}\right]$, such that $P_{s_{0}}$ and $Q_{s_{0}}$ have no non constant common factor in $\mathbb{R}\left[T_{1}, \ldots, T_{r}\right]$, and such that for some $\alpha \geq 0$ and $\beta_{i} \geq 0, \beta \neq$ $(0, \cdots, 0)$, the polynomials $Q_{s_{0}}$ and $1-q^{\alpha} T_{1}^{\beta_{1}} \ldots T_{r}^{\beta_{r}}$ have a non constant common factor in $\mathbb{R}\left[T_{1}, \ldots, T_{r}\right]$. Indeed, otherwise, since one can take $q$ to be transcendental, for every $s_{0}$ in $S$, one could write $\mathcal{M}(\varphi)_{\mid s=s_{0}}$ as a quotient $\frac{P_{s_{0}}}{Q_{s_{0}}}$ of polynomials in $\mathbb{A}[T]$, with $Q_{s_{0}}$ in $\Sigma$. Since the polynomials $Q_{s_{0}}$ all divide a fixed non zero polynomial in $\mathbb{A}[T]$, they have a common multiple $Q$ in $\Sigma$, so we can assume $Q_{s_{0}}$ is independent of $s_{0}$, hence there exists $P$ in $\mathcal{P}(S)\{\{T\}\}$ which gives $P_{s_{0}}$ when evaluated at $s_{0}$ for every $s_{0}$ in $S$, and $\mathcal{M}(\varphi)$ would belong to $\mathcal{P}(S) \llbracket T \rrbracket_{\Sigma}$.

It follows there exists $z_{1}, \ldots, z_{r}$ in $\mathbb{C}$, with $\left|z_{1}\right| \leq 1, \ldots,\left|z_{r}\right| \leq 1$, such that $P_{s_{0}}\left(z_{1}, \ldots, z_{r}\right) \neq 0$ and $Q_{s_{0}}\left(z_{1}, \ldots, z_{r}\right)=0$. In particular the family $\left(\vartheta_{q}\left(\varphi\left(s_{0}, i\right)\right)\right)_{i \in \mathbb{N}^{r}}$ cannot be summable, since the summability of a family of real numbers $\left(a_{i}\right)_{i \in \mathbb{N}^{r}}$ implies that the series $\sum_{i \in \mathbb{N}^{r}} a_{i} z^{i}$ is convergent for every $z=\left(z_{1}, \ldots, z_{r}\right)$ in $\mathbb{C}^{r}$ with $\left|z_{1}\right| \leq 1, \ldots,\left|z_{r}\right| \leq 1$

4.5.5. - We may also characterise $S$-integrability in terms of the $\mathbb{L}$-degree as follows. We consider the unique extension $\operatorname{deg}_{\mathbb{L}}: \mathbb{A} \rightarrow \mathbb{Z} \cup\{-\infty\}$ of the function degree in $\mathbb{L}$ from $\mathbb{Z}[\mathbb{L}]$ to $\mathbb{Z} \cup\{-\infty\}$ which satisfies $\operatorname{deg}_{\mathbb{L}}(a b)=\operatorname{deg}_{\mathbb{L}}(a)+\operatorname{deg}_{\mathbb{L}}(b)$. We have $\operatorname{deg}_{\mathbb{L}}(a+b) \leq \sup \left(\operatorname{deg}_{\mathbb{L}}(a), \operatorname{deg}_{\mathbb{L}}(b)\right)$, with equality if $a$ and $b$ are both in $\mathbb{A}_{+}$. Now if $S$ is a definable set and $\varphi$ is a function in $\mathcal{P}(S)$, we denote by $\operatorname{deg}_{\mathbb{L}}(\varphi)$ the function $S \rightarrow \mathbb{Z} \cup\{-\infty\}$ which sends $s$ to $\operatorname{deg}_{\mathbb{L}}(\varphi(s))$.

4.5.6. Proposition. - The following conditions are equivalent for a function $\varphi$ in $\mathcal{P}\left(S \times \mathbb{Z}^{r}\right)$ :

(i) $\varphi$ is $S$-integrable. 
(ii) For every $s$ in $S, \lim _{|x| \mapsto \infty} \operatorname{deg}_{\mathbb{L}}(\varphi(s, x))=-\infty$, where $|x|$ stands for $\left|x_{1}\right|+$ $\cdots+\left|x_{r}\right|$.

(iii) For every $q>1, \lim _{|x| \mapsto \infty} \vartheta_{q}(\varphi(s, x))=0$.

Proof. - Take $\varphi$ in $\mathcal{P}\left(S \times \mathbb{Z}^{r}\right)$. If $\varphi$ is $S$-integrable, we know by Theorem 4.5.4 that $\mathcal{M}(\varphi)$ is in $\mathcal{P}(S) \llbracket T, T^{-1} \rrbracket_{\Sigma}$. But if $\mathcal{M}(\varphi)$ belongs to $\mathcal{P}(S) \llbracket T, T^{-1} \rrbracket_{\Sigma}$, then the condition that for every $s$ in $S$, $\lim _{|x| \mapsto \infty} \operatorname{deg}_{\mathbb{L}}(\varphi)(s, x)=-\infty$, clearly holds. For the reverse implication, we may by the Cell Decomposition Theorem assume that the support of $\varphi$ is contained in a $S$-cell $A$ and that the restriction of $\varphi$ to $A$ is of the form $\prod_{1 \leq k \leq d} \alpha_{k} \mathbb{L}^{\beta}$ where $\alpha_{k}$ and $\beta$ are $S$-linear functions on $A$ and that furthermore, for fixed $s, \lim _{|x| \mapsto \infty} \beta(s, x)=-\infty$. These conditions clearly imply the summability of the corresponding series. This proves the equivalence of (i) and (ii). The equivalence of (ii) and (iii) is clear.

We have the following statement of Fubini type:

4.5.7. Lemma. - Let $S$ be a definable set and let $\varphi$ be in $\mathcal{P}\left(S \times \mathbb{Z}^{r}\right)$. Write $r=r_{1}+r_{2}$ and identify $\mathbb{Z}^{r}$ with $\mathbb{Z}^{r_{1}} \times \mathbb{Z}^{r_{2}}$.

(1) If $\varphi$ is $S$-integrable, then $\varphi$, as a function in $\mathcal{P}\left(S \times \mathbb{Z}^{r_{1}} \times \mathbb{Z}^{r_{2}}\right)$, is $S \times \mathbb{Z}^{r_{1}}$ integrable, $\mu_{S \times \mathbb{Z}^{r_{1}}}(\varphi)$ is $S$-integrable and

$$
\mu_{S}\left(\mu_{S \times \mathbb{Z}^{r}}(\varphi)\right)=\mu_{S}(\varphi)
$$

(2) Assume $\varphi$ is in $\mathcal{P}_{+}\left(S \times \mathbb{Z}^{r}\right)$. Then $\varphi$ is $S$-integrable if and only if it is $S \times \mathbb{Z}^{r_{1}}$ integrable and $\mu_{S \times \mathbb{Z}^{r_{1}}}(\varphi)$ is $S$-integrable.

Proof. - In view of Theorem-Definition 4.5.1, the statement amounts to the fact that if a family of real numbers $\left(a_{i, j}\right)_{(i, j) \in \mathbb{Z}^{r_{1}} \times \mathbb{Z}^{r_{2}}}$ is summable then, for every $i$, $\left(a_{i, j}\right)_{j \in \mathbb{Z}^{r_{2}}}$ is summable, the family $\left(b_{i}=\sum_{j \in \mathbb{Z}^{r_{2}}} a_{i, j}\right)_{i \in \mathbb{Z}^{r_{1}}}$ is summable, $\sum_{i \in \mathbb{Z}^{r_{1}}} b_{j}=$

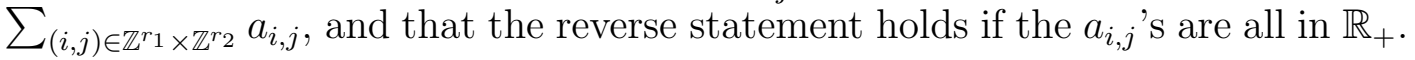

Let $\lambda: S \times \mathbb{Z}^{r} \rightarrow S \times \mathbb{Z}^{s}$ be a definable function commuting with the projections to $S$. Let $Z$ be a definable subset of $S \times \mathbb{Z}^{r}$ on which $\lambda$ is injective. Let $\varphi$ be a function in $\mathcal{P}\left(S \times \mathbb{Z}^{r}\right)$ which is zero outside $Z$. We define the function $\lambda_{+}(\varphi)$ on $S \times \mathbb{Z}^{s}$ by $\lambda_{+}(\varphi)(\lambda(s, i))=\varphi(s, i)$ and $\lambda_{+}(\varphi)(s, j)=0$ if $(s, j)$ does not lie in the image of $\lambda$. Clearly $\lambda_{+}(\varphi)$ lies in $\mathcal{P}\left(S \times \mathbb{Z}^{s}\right)$.

The following statement will be useful in the proof of the change of variable formula.

4.5.8. Lemma. - Let $S$ be a definable set and let $\lambda: S \times \mathbb{Z}^{r} \rightarrow S \times \mathbb{Z}^{s}$ be a definable function commuting with the projections to $S$. Let $Z$ be a definable subset of $S \times \mathbb{Z}^{r}$ on which $\lambda$ is injective. Let $\varphi$ be a function in $\mathcal{P}\left(S \times \mathbb{Z}^{r}\right)$ which is zero outside $Z$. Then $\varphi$ is $S$-integrable if and only if $\lambda_{+}(\varphi)$ is $S$-integrable. Furthermore, if these conditions hold, then

$$
\mu_{S}(\varphi)=\mu_{S}\left(\lambda_{+}(\varphi)\right)
$$

Proof. - The first statement follows directly from the definition of $S$-integrability and the second from Theorem-Definition 4.5.1. 
4.6. Generalization: From Presburger sets to definable subassignments. - Note that any Presburger subset $S$ of $\mathbb{Z}^{m}$ is clearly also $\mathcal{L}_{\mathrm{DP}, \mathrm{P}}$-definable. Furthermore, it follows from the Denef-Pas quantifier elimination Theorem 2.1.1 that a function $f: S \rightarrow \mathbb{Z}$ is $\mathcal{L}_{\mathrm{DP}, \mathrm{P}}$-definable if and only if it is $\mathbf{L}_{\mathrm{PR}}$-definable.

Let us generalize what we did in 4.1,4.5 for Presburger subsets to definable subassignments in $\operatorname{GDef}_{k}\left(\mathcal{L}_{\mathrm{DP}, \mathrm{P}}\right)$.

Let $S$ be a definable subassignment in $\operatorname{GDef}_{k}\left(\mathcal{L}_{\mathrm{DP}, \mathrm{P}}\right)$. We denote by $|S|$ its set of points, defined in 2.6 (this is indeed a set by 2.3). Note that to any definable morphism $\alpha: S \rightarrow h[0,0,1]$ corresponds a function $\tilde{\alpha}:|S| \rightarrow \mathbb{Z}$. (Since $\alpha$ and $\tilde{\alpha}$ determine each other we shall not distinguish their notation after 4.6.) We define the ring $\mathcal{P}(S)$ of constructible Presburger functions on $S$ as the subring of the ring of functions $|S| \rightarrow \mathbb{A}$ generated by constant functions $|S| \rightarrow \mathbb{A}$, and by functions $\tilde{\alpha}:|S| \rightarrow \mathbb{Z}$ and $\mathbb{L}^{\tilde{\beta}}:|S| \rightarrow \mathbb{A}$ for definable morphisms $\alpha, \beta: S \rightarrow h[0,0,1]$. We also denote by $\mathcal{P}_{+}(S)$ the semiring of functions in $\mathcal{P}(S)$ with values in $\mathbb{A}_{+}$. Everything we did in 4.1,4.5, including the proof of Theorem 4.3.2, generalizes mutatis mutandis to that more general situation, up to minor changes like replacing $S \times \mathbb{Z}^{r}$ by $S \times h_{\mathbb{Z}^{r}}=$ $S[0,0, r]$ or $s$ in $S$ by $s$ in $|S|$. To give an example of this adaptation, an element $\varphi$ of $\mathcal{P}(S[0,0, r])$ lies in $\mathrm{I}_{S} \mathcal{P}(S[0,0, r])$ if and only if, for every $s$ in $|S|$, the family $(\varphi(s, i))_{i \in \mathbb{Z}^{r}}$ is summable in the sense of 4.5. Thus it is allowed to use constructions and results in $4.1+4.5$ for definable subassignments in $\operatorname{GDef}_{k}\left(\mathcal{L}_{\mathrm{DP}, \mathrm{P}}\right)$ in the rest of the paper by referring to the corresponding ones for Presburger sets.

Note that $h_{\mathbb{Z}^{r}}=h[0,0, r]$ has more points than $\mathbb{Z}^{r}$ since a point on $h[0,0, r]$ consists of a tuple $(a, K)$ with $K$ in Field $k$ and $a \in \mathbb{Z}^{r}$. Likewise, the ring $\mathcal{P}(h[0,0, r])$ is larger than the ring $\mathcal{P}\left(\mathbb{Z}^{r}\right)$ defined in 4.2, since it contains $\mathcal{P}\left(\mathbb{Z}^{r}\right)$ and is generated as a ring by $\mathcal{P}\left(\mathbb{Z}^{r}\right)$ and characteristic functions of definable subassignments of the final object $h_{\mathrm{Spec} k}$.

\section{Constructible motivic functions}

From now on and until the end of the paper, we shall work with $\mathcal{L}_{\mathrm{DP}}=\mathcal{L}_{\mathrm{DP}, \mathrm{P}}$ as Denef-Pas language.

5.1. Grothendieck rings and semirings. - In previous publications on motivic integration, free abelian groups of varieties (or Chow motives) over $k$ were used to build up Grothendieck rings. Here we shall consider a $\mathcal{L}_{\mathrm{DP}}$-variant using definable subassignments, which has several advantages, in particular, of being "universal" in our setting. In the absolute case, one has natural ring morphisms to the previous Grothendieck rings (cf. section [16). Since in integration theory "positive" functions play an important role, we shall also consider Grothendieck semirings. We first recall some basics from the theory of semirings.

5.1.1. Semirings. - Let us recall that a (commutative) semiring $A$ is a set equipped with two operations: addition and multiplication. With respect to addition $A$ is a 
commutative semigroup (monoid) with 0 as unit element. With respect to multiplication $A$ is a commutative semigroup with 1 as a unit element. Furthermore the two structures are connected by the axioms $x(y+z)=x y+x z$ and $0 x=0$. A morphism of semirings is a mapping compatible with the unit elements and the operations. A module (or semimodule) over a semiring $A$ is a commutative semigroup $M$ with an operation $\cdot A \times M \rightarrow A$ satisfying the familiar axioms $(a b) \cdot m=a \cdot(b \cdot m)$, $(a+b) \cdot m=a \cdot m+b \cdot m, a \cdot(m+n)=a \cdot m+a \cdot n, 0 \cdot m=0, a \cdot 0=0$ and $1 \cdot m=m$. One defines morphisms of $A$-modules in the usual way. Also, if $M$ and $N$ are $A$-modules, one can define their tensor product $M \otimes_{A} N$ in the usual way using generators and relations. It is an $A$-module representing the functor of bilinear morphisms on $M \times N$ and its existence also follows from classical representability results. If $B$ is an $A$-algebra (that is, a semiring together with a morphism $A \rightarrow B$ ), for every $A$-module $M$ the module $B \otimes_{A} M$ has a natural $B$-module structure compatible with the $A$-module structure. Also, if $B$ and $C$ are $A$-algebras, the formula $\sum_{i}\left(b_{i} \otimes c_{i}\right) \sum_{j}\left(b_{j}^{\prime} \otimes c_{j}^{\prime}\right)=\sum_{i, j} b_{i} b_{j}^{\prime} \otimes c_{i} c_{j}^{\prime}$ endowes $B \otimes_{A} C$ with a structure of $A$-algebra.

5.1.2. Definition and properties of RDef. - Let $Z$ be a definable subassignment in $\operatorname{GDef}_{k}$. We shall use in a essential way the full subcategory $\operatorname{RDef}_{Z}$ of $\operatorname{GDef}_{Z}$, whose objects are definable subassignments $Y$ of $Z \times h_{\mathbb{A}_{k}^{n}}$, for some $n$, the morphism $Y \rightarrow Z$ being the one induced by projection on the $Z$ factor. If $Y$ and $Y^{\prime}$ are two objects of $\operatorname{RDef}_{Z}$, their fiber product $Y \otimes_{Z} Y^{\prime}$ together with the canonical morphism $Y \otimes_{Z} Y^{\prime} \rightarrow Z$ yields an object of $\operatorname{RDef}_{Z}$.

It is this category $\operatorname{RDef}_{Z}$, and not $\operatorname{Def}_{Z}$, that is used to built relative Grothendieck rings over $Z$ with. This is so because we want the Grothendieck rings to capture information over the residue fields, not over the valued field nor the integers.

We define the Grothendieck semigroup $S K_{0}\left(\operatorname{RDef}_{Z, k}\left(\mathcal{L}_{\mathrm{DP}}\right)\right)$ - or $S K_{0}\left(\mathrm{RDef}_{Z}\right)$ for short -, as the quotient of the free abelian semigroup over symbols $[Y \rightarrow Z]$ with $Y \rightarrow Z$ in $\operatorname{RDef}_{Z}$ by relations

$$
\begin{gathered}
{[\emptyset \rightarrow Z]=0,} \\
{[Y \rightarrow Z]=\left[Y^{\prime} \rightarrow Z\right]}
\end{gathered}
$$

if $Y \rightarrow Z$ is isomorphic to $Y^{\prime} \rightarrow Z$ and

$$
\left[\left(Y \cup Y^{\prime}\right) \rightarrow Z\right]+\left[\left(Y \cap Y^{\prime}\right) \rightarrow Z\right]=[Y \rightarrow Z]+\left[Y^{\prime} \rightarrow Z\right]
$$

for $Y$ and $Y^{\prime}$ definable subassignments of some $Z[0, n, 0] \rightarrow Z$. Similarly one defines the Grothendieck group $K_{0}\left(\operatorname{RDef}_{Z, k}\left(\mathcal{L}_{\mathrm{DP}}\right)\right)$, or $K_{0}\left(\operatorname{RDef}_{Z}\right)$ for short, as the quotient of the free abelian group over symbols $[Y \rightarrow Z]$ with $Y \rightarrow Z$ in $\operatorname{RDef}_{Z}$ by relations (5.1.2) and (5.1.3). Cartesian fiber product over $Z$ induces a natural semiring, resp. ring, structure on $S K_{0}\left(\operatorname{RDef}_{Z}\right)$, resp. $K_{0}\left(\operatorname{RDef}_{Z}\right)$, by setting

$$
[Y \rightarrow Z]\left[Y^{\prime} \rightarrow Z\right]=\left[Y \otimes_{Z} Y^{\prime} \rightarrow Z\right]
$$

Let us remark that $[Z \rightarrow Z]$ is the multiplicative unit and that $K_{0}\left(\operatorname{RDef}_{Z}\right)$ is nothing but the ring obtained from $S K_{0}\left(\operatorname{RDef}_{Z}\right)$ by inverting additively every element. 
However note that there is no reason for the canonical morphism $S K_{0}\left(\operatorname{RDef}_{Z}\right) \rightarrow$ $K_{0}\left(\operatorname{RDef}_{Z}\right)$ to be injective in general.

We extend some operations from section 2, If $f$ is a morphism $Z \rightarrow Z^{\prime}$ in GDef $_{k}$, then the functor $f^{*}$, as defined in 2.4, induces a semiring morphism $f^{*}: S K_{0}\left(\operatorname{RDef}_{Z^{\prime}}\right) \rightarrow S K_{0}\left(\operatorname{RDef}_{Z}\right)$ and a ring morphism $f^{*}: K_{0}\left(\operatorname{RDef}_{Z^{\prime}}\right) \rightarrow$ $K_{0}\left(\operatorname{RDef}_{Z}\right)$. Also, if $z$ is a point of $Z^{\prime}$, then the functor $i_{z}^{*}$, as defined in 2.6, induces a semiring morphism $i_{z}^{*}: S K_{0}\left(\operatorname{RDef}_{Z^{\prime}}\right) \rightarrow S K_{0}\left(\operatorname{RDef}_{k(z)}\right)$ and a ring morphism $i_{z}^{*}: K_{0}\left(\operatorname{RDef}_{Z^{\prime}}\right) \rightarrow K_{0}\left(\operatorname{RDef}_{k(z)}\right)$. Note that $f^{*} \circ g^{*}=(g \circ f)^{*}$ for composable morphisms $f, g$ in $\operatorname{GDef}_{k}$.

Constructing a direct image functor $f_{!}$(on constructible functions) for general morphisms $f: X \rightarrow Y$ in $\operatorname{Def}_{Y}$ is one of the main purposes of the paper. However, on the level of $\operatorname{RDef}_{Y}$ and its Grothendieck rings, with $f$ a morphism in $\operatorname{RDef}_{Y}, f_{\text {! }}$ is easy to define and is merely a universal operator. Namely, if $f: X \rightarrow Y$ is a morphism $\operatorname{RDef}_{Y}$, the functor $f_{!}$, as defined in 2.4, restricts to a functor $f_{!}: \operatorname{RDef}_{X} \rightarrow \operatorname{RDef}_{Y}$ and this induces a semiring morphism $f_{!}: S K_{0}\left(\operatorname{RDef}_{X}\right) \rightarrow S K_{0}\left(\operatorname{RDef}_{Y}\right)$ and a ring morphism $f_{!}: K_{0}\left(\operatorname{RDef}_{X}\right) \rightarrow K_{0}\left(\operatorname{RDef}_{Y}\right)$. Note that $g_{!} \circ f_{!}=(g \circ f)$ ! for composable such morphisms. It is also clear that the projection formula $f_{!}\left(x f^{*}(y)\right)=f_{!}(x) y$ holds for $x$ in $S K_{0}\left(\operatorname{RDef}_{X}\right)\left(\right.$ resp. $\left.K_{0}\left(\operatorname{RDef}_{X}\right)\right)$ and $y$ in $S K_{0}\left(\operatorname{RDef}_{Y}\right)\left(\operatorname{resp} . K_{0}\left(\operatorname{RDef}_{Y}\right)\right)$.

Note that any $a$ in $S K_{0}\left(\operatorname{RDef}_{Z}\right)$ is of the form $[\pi: Y \rightarrow Z]$, with $Y$ in $\operatorname{RDef}_{Z}$ and $\pi$ the projection. The definable subassignment $\pi(Y)$ depends only on $a$ and we denote it $\operatorname{Supp}(a)$. Note that, by definition, a point $z$ of $Z$ is a point of $\operatorname{Supp}(a)$ if and only if $i_{z}^{*}(a)$ is different from the empty subassignment. Also, for $a$ and $b$ in $S K_{0}\left(\operatorname{RDef}_{Z}\right)$, we have $\operatorname{Supp}(a+b)=\operatorname{Supp}(a) \cup \operatorname{Supp}(b)$ and $\operatorname{Supp}(a b)=\operatorname{Supp}(a) \cap \operatorname{Supp}(b)$.

5.2. Constructible Presburger functions. - In 4.6, we assigned to every $Z$ in GDef $_{k}$ the ring $\mathcal{P}(Z)$ of constructible Presburger functions on $Z$. If $f: Z \rightarrow Y$ is a morphism in GDef $_{k}$, composition with $f$ yields natural morphisms $f^{*}: \mathcal{P}(Y) \rightarrow$ $\mathcal{P}(Z)$ and $f^{*}: \mathcal{P}_{+}(Y) \rightarrow \mathcal{P}_{+}(Z)$, namely, by sending $\varphi$ to $\varphi \circ f$. Similarly, if $z$ is point of $Z$ we have morphisms $i_{z}^{*}: \mathcal{P}(Z) \rightarrow \mathcal{P}\left(h_{\text {Spec } k(z)}\right)$ and $i_{z}^{*}: \mathcal{P}_{+}(Z) \rightarrow \mathcal{P}_{+}\left(h_{\text {Spec } k(z)}\right)$.

For $Y$ a definable subassignment of $Z$, we denote by $\mathbf{1}_{Y}$ the function in $\mathcal{P}(Z)$ with value 1 on $Y$ and zero on $Z \backslash Y$. We shall denote by $\mathcal{P}^{0}(Z)\left(\operatorname{resp} . \mathcal{P}_{+}^{0}(Z)\right)$ the subring (resp. subsemiring) of $\mathcal{P}(Z)$ (resp. $\mathcal{P}_{+}(Z)$ ) generated by the functions $\mathbf{1}_{Y}$ for all definable subassignments $Y$ of $Z$ and by the constant function $\mathbb{L}-1$.

Let us denote by $\mathbb{L}_{Z}=\mathbb{L}$ the class of $Z \times h_{\mathbb{A}_{k}^{1}}$ in $K_{0}\left(\operatorname{RDef}_{Z}\right)$ and in $S K_{0}\left(\operatorname{RDef}_{Z}\right)$. We also denote by $\mathbb{L}_{Z}-1=\mathbb{L}-1$ the class of $Z \times h_{\mathbb{A}_{k}^{1} \backslash\{0\}}$ in $S K_{0}\left(\operatorname{RDef}_{Z}\right)$. Note that $(\mathbb{L}-1)+1=\mathbb{L}$ in $S K_{0}\left(\operatorname{RDef}_{Z}\right)$.

We have a canonical ring, resp. semiring, morphism $\mathcal{P}^{0}(Z) \rightarrow K_{0}\left(\operatorname{RDef}_{Z}\right)$, resp. $\mathcal{P}_{+}^{0}(Z) \rightarrow S K_{0}\left(\operatorname{RDef}_{Z}\right)$, sending $\mathbf{1}_{Y}$ to $[i: Y \rightarrow Z]$, with $i$ the inclusion, and $\mathbb{L}-1$ to $\mathbb{L}-1$.

5.2.1. Proposition. - Let $S$ be in $\mathrm{GDef}_{k}$.

(1) Let $W$ be a definable subassignment of $h[0, n, 0]$. The canonical morphisms

$$
\mathcal{P}(S) \otimes_{\mathcal{P}^{0}(S)} \mathcal{P}^{0}(S \times W) \longrightarrow \mathcal{P}(S \times W)
$$


and

$$
\mathcal{P}_{+}(S) \otimes_{\mathcal{P}_{+}^{0}(S)} \mathcal{P}_{+}^{0}(S \times W) \longrightarrow \mathcal{P}_{+}(S \times W)
$$

are isomorphisms.

(2) Let $W$ be a definable subassignment of $h[0,0, r]$. The canonical morphisms

$$
K_{0}\left(\operatorname{RDef}_{S}\right) \otimes_{\mathcal{P}^{0}(S)} \mathcal{P}^{0}(S \times W) \longrightarrow K_{0}\left(\operatorname{RDef}_{S \times W}\right)
$$

and

$$
S K_{0}\left(\operatorname{RDef}_{S}\right) \otimes_{\mathcal{P}_{+}^{0}(S)} \mathcal{P}_{+}^{0}(S \times W) \longrightarrow S K_{0}\left(\operatorname{RDef}_{S \times W}\right)
$$

are isomorphisms.

Proof. — Follows directly from Theorem 2.1.1.

5.3. Constructible motivic functions. - Let $Z$ be a definable subassignment in $\operatorname{GDef}_{k}$. We define the semiring $\mathscr{C}_{+}(Z)$ of positive constructible motivic functions on $Z$ as

$$
\mathscr{C}_{+}(Z):=S K_{0}\left(\operatorname{RDef}_{Z}\right) \otimes_{\mathcal{P}_{+}^{0}(Z)} \mathcal{P}_{+}(Z)
$$

Similarly we define the ring $\mathscr{C}(Z)$ of constructible motivic functions on $Z$ as

$$
\mathscr{C}(Z):=K_{0}\left(\operatorname{RDef}_{Z}\right) \otimes_{\mathcal{P}^{0}(Z)} \mathcal{P}(Z) .
$$

Let us remark that $\mathscr{C}(Z)$ is nothing but the ring obtained from $\mathscr{C}_{+}(Z)$ by inverting additively every element and that in general there is no reason for the canonical morphism $\mathscr{C}_{+}(Z) \rightarrow \mathscr{C}(Z)$ to be injective.

For $\varphi$ and $\varphi^{\prime}$ in $\mathscr{C}_{+}(Z)$, we shall write $\varphi \geq \varphi^{\prime}$ if $\varphi=\varphi^{\prime}+\varphi^{\prime \prime}$ for some $\varphi^{\prime \prime}$ in $\mathscr{C}_{+}(Z)$.

5.3.1. Proposition. - Let $S$ be in GDef $_{k}$.

(1) Let $W$ be a definable subassignment of $h[0, n, 0]$. The canonical morphisms

$$
\mathcal{P}(S) \otimes_{\mathcal{P}^{0}(S)} K_{0}\left(\operatorname{RDef}_{S \times W}\right) \longrightarrow \mathscr{C}(S \times W)
$$

and

$$
\mathcal{P}_{+}(S) \otimes_{\mathcal{P}_{+}^{0}(S)} S K_{0}\left(\operatorname{RDef}_{S \times W}\right) \longrightarrow \mathscr{C}_{+}(S \times W)
$$

are isomorphisms.

(2) Let $W$ be a definable subassignment of $h[0,0, r]$. The canonical morphisms

$$
K_{0}\left(\operatorname{RDef}_{S}\right) \otimes_{\mathcal{P}^{0}(S)} \mathcal{P}(S \times W) \longrightarrow \mathscr{C}(S \times W)
$$

and

$$
S K_{0}\left(\operatorname{RDef}_{S}\right) \otimes_{\mathcal{P}_{+}^{0}(S)} \mathcal{P}_{+}(S \times W) \longrightarrow \mathscr{C}_{+}(S \times W)
$$

are isomorphisms.

Proof. - Direct consequence of Proposition 5.2.1.

Note that $\mathscr{C}\left(h_{\text {Spec } k}\right)$ is canonically isomorphic to $K_{0}\left(\operatorname{RDef}_{k}\right) \otimes_{\mathbb{Z}[\mathbb{L}]} \mathbb{A}$ and that $\mathscr{C}_{+}\left(h_{\text {Spec } k}\right)$ is canonically isomorphic to $S K_{0}\left(\operatorname{RDef}_{k}\right) \otimes_{\mathbb{N}[\mathbb{L}-1]} \mathbb{A}_{+}$. 
5.4. Inverse image of constructible motivic functions. - Let $f: Z \rightarrow Y$ be a morphism in $\operatorname{GDef}_{k}$. Since $f^{*}$ as defined on $\mathcal{P}(Y)$ and $K_{0}\left(\operatorname{RDef}_{Y}\right)$ is compatible with the morphism $\mathcal{P}^{0}(Y) \rightarrow K_{0}\left(\operatorname{RDef}_{Y}\right)$, one gets by tensor product an inverse image morphism $f^{*}: \mathscr{C}(Y) \rightarrow \mathscr{C}(Z)$. One defines similarly $f^{*}: \mathscr{C}_{+}(Y) \rightarrow \mathscr{C}_{+}(Z)$. Clearly $f^{*} \circ g^{*}=(g \circ f)^{*}$ and $\mathrm{id}^{*}=\mathrm{id}$.

Similarly, if $z$ is a point of $Z$, there are natural extensions $i_{z}^{*}: \mathscr{C}(Z) \rightarrow$ $\mathscr{C}\left(h_{\text {Spec } k(z)}\right)$ and $i_{z}^{*}: \mathscr{C}_{+}(Z) \rightarrow \mathscr{C}_{+}\left(h_{\text {Spec } k(z)}\right)$ of the restrictions $i_{z}^{*}$ already defined.

If $Z_{1}$ and $Z_{2}$ are disjoint definable subassignments of some $h_{W}$, then

$$
\mathscr{C}_{+}\left(Z_{1} \cup Z_{2}\right) \simeq \mathscr{C}_{+}\left(Z_{1}\right) \oplus \mathscr{C}_{+}\left(Z_{2}\right) \quad \text { and } \quad \mathscr{C}\left(Z_{1} \cup Z_{2}\right) \simeq \mathscr{C}\left(Z_{1}\right) \oplus \mathscr{C}\left(Z_{2}\right) .
$$

If $Z_{1}$ and $Z_{2}$ are in $\operatorname{GDef}_{S}$, then we have canonical morphisms

$\mathscr{C}_{+}\left(Z_{1}\right) \otimes_{\mathscr{C}_{+}(S)} \mathscr{C}_{+}\left(Z_{2}\right) \rightarrow \mathscr{C}_{+}\left(Z_{1} \times{ }_{S} Z_{2}\right) \quad$ and $\mathscr{C}\left(Z_{1}\right) \otimes_{\mathscr{C}(S)} \mathscr{C}\left(Z_{2}\right) \rightarrow \mathscr{C}\left(Z_{1} \times{ }_{S} Z_{2}\right)$.

5.5. Push-forward for inclusions. - Let $i: Z \hookrightarrow Z^{\prime}$ be an inclusion between two definable subassignments of $h_{W}$. Composition with $i$ yields morphisms $i_{!}$: $K_{0}\left(\operatorname{RDef}_{Z}\right) \rightarrow K_{0}\left(\operatorname{RDef}_{Z^{\prime}}\right)$ and $i_{!}: S K_{0}\left(\operatorname{RDef}_{Z}\right) \rightarrow S K_{0}\left(\operatorname{RDef}_{Z^{\prime}}\right)$. Extension by zero induces morphisms $i_{!}: \mathcal{P}(Z) \rightarrow \mathcal{P}\left(Z^{\prime}\right)$ and $i_{!}: \mathcal{P}_{+}(Z) \rightarrow \mathcal{P}_{+}\left(Z^{\prime}\right)$. Since they are compatible on $\mathcal{P}^{0}(Z)$ and $\mathcal{P}_{+}^{0}(Z)$, we get morphisms $i_{!}: \mathscr{C}(Z) \rightarrow \mathscr{C}\left(Z^{\prime}\right)$ and $i_{!}: \mathscr{C}_{+}(Z) \rightarrow \mathscr{C}_{+}\left(Z^{\prime}\right)$ by tensor product.

5.6. Push-forward for $k$-projections. - Let $S$ be a definable subassignment in $\mathrm{GDef}_{k}$ and consider the projection $f: S[0, n, 0] \rightarrow S$ on the first factor. Recall that, by Proposition [5.3.1, we have a canonical isomorphism $\mathscr{C}(S[0, n, 0]) \simeq K_{0}\left(\operatorname{RDef}_{S[0, n, 0]}\right) \otimes_{\mathcal{P}^{0}(S)} \mathcal{P}(S)$, so that we may define a ring morphism $f_{!}: \mathscr{C}(S[0, n, 0]) \rightarrow \mathscr{C}(S)$ by sending $\sum_{i} a_{i} \otimes \varphi_{i}$ to $\sum_{i} f_{!}\left(a_{i}\right) \otimes \varphi_{i}$, with $a_{i}$ in $K_{0}\left(\operatorname{RDef}_{S[0, n, 0]}\right), \varphi_{i}$ in $\mathcal{P}(S)$, and $f_{!}\left(a_{i}\right)$ as in section [5.1.2, this is clearly independent of the choices. We define a semiring morphism $f_{!}: \mathscr{C}_{+}(S[0, n, 0]) \rightarrow \mathscr{C}_{+}(S)$ in the same way. Clearly these morphisms satisfy the projection formula

$$
f_{!}\left(x f^{*}(y)\right)=f_{!}(x) y
$$

for $x$ in $\mathscr{C}(S[0, n, 0])$, resp. $\mathscr{C}_{+}(S[0, n, 0])$, and $y$ in $\mathscr{C}(S)$, resp. $\mathscr{C}_{+}(S)$.

5.7. Rational series and integrability. — Let $S$ be a definable subassignment in $\mathrm{GDef}_{k}$. As in 4.4, we consider the power series ring $\mathscr{C}(S) \llbracket T \rrbracket=\mathscr{C}(S) \llbracket T_{1}, \ldots, T_{r} \rrbracket$ and $\mathscr{C}(S) \llbracket T, T^{-1} \rrbracket=\mathscr{C}(S) \llbracket T_{1}, \ldots, T_{r}, T_{1}^{-1}, \ldots, T_{r}^{-1} \rrbracket$. We shall set $\mathscr{C}(S) \llbracket T \rrbracket_{\Gamma}:=$ $\mathscr{C}(S) \otimes_{\mathcal{P}(S)} \mathcal{P}(S) \llbracket T \rrbracket_{\Gamma}$ and $\mathscr{C}(S) \llbracket T, T^{-1} \rrbracket_{\Gamma}:=\mathscr{C}(S) \otimes_{\mathcal{P}(S)} \mathcal{P}(S) \llbracket T, T^{-1} \rrbracket_{\Gamma}$ and view them as $\mathscr{C}(S)$-submodules of $\mathscr{C}(S) \llbracket T \rrbracket$ and $\mathscr{C}(S) \llbracket T, T^{-1} \rrbracket$, respectively.

Now, for $\varphi$ in $\mathscr{C}(S[0,0, r])$ and $i$ in $\mathbb{Z}^{r}$, we denote by $\varphi_{i}$ the restriction of $\varphi$ to $S \times\{i\}$, viewed as an element in $\mathscr{C}(S)$, and, as in 4.4, we set

$$
\mathcal{M}(\varphi):=\sum_{i \in \mathbb{Z}^{r}} \varphi_{i} T^{i}
$$

in $\mathscr{C}(S) \llbracket T, T^{-1} \rrbracket$. 
By (2) of Proposition 5.3.1, we have a canonical isomorphism

$$
\mathscr{C}(S[0,0, r]) \simeq \mathscr{C}(S) \otimes_{\mathcal{P}(S)} \mathcal{P}(S[0,0, r]) .
$$

Since, by the extension of Theorem 4.4.1 to the definable subassignment setting, we have an isomorphism of $\mathcal{P}(S)$-modules

$$
\mathcal{M}: \mathcal{P}(S[0,0, r]) \longrightarrow \mathcal{P}(S) \llbracket T, T^{-1} \rrbracket_{\Gamma},
$$

we get by tensoring with $\mathscr{C}(S)$ the following general rationality statement.

5.7.1. Theorem. - Let $S$ be a definable subassignment in GDef $_{k}$. The mapping $\varphi \mapsto \mathcal{M}(\varphi)$ induces a ring isomorphism

$$
\mathcal{M}: \mathscr{C}(S[0,0, r]) \longrightarrow \mathscr{C}(S) \llbracket T, T^{-1} \rrbracket_{\Gamma} .
$$

Similarly, we define the $\mathscr{C}(S)$-modules

$$
\mathrm{I}_{S} \mathscr{C}(S[0,0, r]):=\mathscr{C}(S) \otimes_{\mathcal{P}(S)} \mathrm{I}_{S} \mathcal{P}(S[0,0, r])
$$

and

We also define

$$
\mathscr{C}(S) \llbracket T, T^{-1} \rrbracket_{\Sigma}:=\mathscr{C}(S) \otimes_{\mathcal{P}(S)} \mathcal{P}(S) \llbracket T, T^{-1} \rrbracket_{\Sigma}
$$

$$
\mathrm{I}_{S} \mathscr{C}_{+}(S[0,0, r]):=\mathscr{C}_{+}(S) \otimes_{\mathcal{P}_{+}(S)} \mathrm{I}_{S} \mathcal{P}_{+}(S[0,0, r]),
$$

where $\mathrm{I}_{S} \mathcal{P}_{+}(S[0,0, r])$ stands for the $\mathcal{P}_{+}(S)$-module $\mathcal{P}_{+}(S[0,0, r]) \cap \mathrm{I}_{S} \mathscr{C}(S[0,0, r])$. A function in $\mathscr{C}(S[0,0, r])$ (resp. $\left.\mathscr{C}_{+}(S[0,0, r])\right)$ will be called $S$-integrable if it belongs to $\mathrm{I}_{S} \mathscr{C}(S[0,0, r])\left(\right.$ resp. $\left.\mathrm{I}_{S} \mathscr{C}_{+}(S[0,0, r])\right)$.

By Theorem 4.5 .4 and tensoring with $\mathscr{C}(S)$, the isomorphism $\mathcal{M}$ induces an isomorphism of $\mathscr{C}(S)$-modules

$$
\mathcal{M}: \mathrm{I}_{S} \mathscr{C}(S[0,0, r]) \longrightarrow \mathscr{C}(S) \llbracket T, T^{-1} \rrbracket_{\Sigma}
$$

By tensoring the morphism of $\mathcal{P}(S)$-modules $\mu_{S}: \mathrm{I}_{S} \mathcal{P}(S[0,0, r]) \rightarrow \mathcal{P}(S)$ with $\mathscr{C}(S)$, we get a morphism of $\mathscr{C}(S)$-modules

$$
\mu_{S}: \mathrm{I}_{S} \mathscr{C}(S[0,0, r]) \longrightarrow \mathscr{C}(S)
$$

Similarly we have a morphism of $\mathscr{C}_{+}(S)$-modules

$$
\mu_{S}: \mathrm{I}_{S} \mathscr{C}_{+}(S[0,0, r]) \longrightarrow \mathscr{C}_{+}(S) .
$$

5.7.2. Proposition. - Let $S$ be a definable subassignment in GDef $_{k}$ and let $\varphi$ be in $\mathscr{C}\left(S \times h_{\mathbb{Z}^{r}}\right)$. Write $r=r_{1}+r_{2}$ and identify $h_{\mathbb{Z}^{r}}$ with $h_{\mathbb{Z}^{r_{1}}} \times h_{\mathbb{Z}^{r_{2}}}$. If $\varphi$ is $S$-integrable, then $\varphi$, as a function in $\mathscr{C}\left(S \times h_{\mathbb{Z}^{r_{1}}} \times h_{\mathbb{Z}^{r_{2}}}\right)$, is $S \times h_{\mathbb{Z}^{r_{1}}}$-integrable, $\mu_{S \times h_{\mathbb{Z}^{r}}}(\varphi)$ is S-integrable and

$$
\mu_{S}\left(\mu_{S \times h_{\mathbb{Z}^{r_{1}}}}(\varphi)\right)=\mu_{S}(\varphi) .
$$

The statement with $\mathscr{C}$ replaced by $\mathscr{C}_{+}$holds also.

Proof. - Follows directly from Lemma 4.5.7 (1). 
Let $\lambda: S[0,0, r] \rightarrow S[0,0, s]$ be a morphism in $\operatorname{Def}_{S}$. Let $\varphi$ be a function in $\mathscr{C}(S[0,0, r])$, resp. $\mathscr{C}_{+}(S[0,0, r])$. Assume $\varphi=\mathbf{1}_{Z} \varphi$ with $Z$ a definable subassignment of $S[0,0, r]$ on which $\lambda$ is injective. Recall that this means that the function $\lambda(K)$ is injective on $Z(K)$ for each $K$ in Field $_{k}$, cf. section 2.2. Thus $\lambda$ restricts to an isomorphism $\lambda^{\prime}$ between $Z$ and $Z^{\prime}:=\lambda(Z)$. We set $\lambda_{+}\left(\mathbf{1}_{Z} \varphi\right):=\left[i_{!}^{\prime}\left(\lambda^{\prime-1}\right)^{*} i^{*}\right](\varphi)$ in $\mathscr{C}(S[0,0, r])$, resp. in $\mathscr{C}_{+}(S[0,0, r])$, where $i$ and $i^{\prime}$ denote respectively the inclusions of $Z$ and $Z^{\prime}$ in $S[0,0, r]$ and $S[0,0, s]$. Clearly this definition does not depend on the choice of $Z(6)$.

The following statement follows directly from Lemma 4.5.8.

5.7.3. Proposition. - Let $\lambda: S[0,0, r] \rightarrow S[0,0, s]$ be a morphism in GDef $_{S}$. Assume $\lambda$ is injective on a definable subassignment $Z$ of $S[0,0, r]$. Let $\varphi$ be a function in $\mathscr{C}(S[0,0, r])$ such that $\mathbf{1}_{Z} \varphi=\varphi$. Then $\varphi$ is $S$-integrable if and only if $\lambda_{+}(\varphi)$ is $S$ integrable. If these conditions are satisfied then $\mu_{S}(\varphi)=\mu_{S}\left(\lambda_{+}(\varphi)\right)$. The statement with $\mathscr{C}$ replaced by $\mathscr{C}_{+}$holds also.

5.8. Positivity and Fubini. - Let $S$ be a definable subassignment in GDef $_{k}$. It is quite clear that if $f$ and $g$ are in $\mathcal{P}_{+}(S[0,0, r]), f \geq g$ and $f$ is $S$-integrable, then $g$ is $S$-integrable. We shall now prove a similar statement for $\mathscr{C}_{+}$. 5.1 .2

For $a$ in $S K_{0}\left(\operatorname{RDef}_{S}\right)$, we shall write $\mathbf{1}_{a}:=\mathbf{1}_{\operatorname{Supp}(a)}$, with $\operatorname{Supp}(a)$ as defined in

5.8.1. Proposition. - Let $S$ be a definable subassignment in GDef $_{k}$ and let $f$ be a function in $\mathscr{C}_{+}(S[0,0, r])$. Write $f=\sum_{i} a_{i} \otimes \varphi_{i}$, with $a_{i}$ in $S K_{0}\left(\operatorname{RDef}_{S}\right)$ and $\varphi_{i}$ in $\mathcal{P}_{+}(S[0,0, r])$. Then $f$ is $S$-integrable if and only every function $\mathbf{1}_{a_{i}} \varphi_{i}$ is $S$-integrable.

Proof. - Let $f$ be a $S$-integrable function in $\mathscr{C}_{+}(S[0,0, r])$. Write $f=\sum_{i} a_{i} \otimes \varphi_{i}$, with $a_{i}$ in $S K_{0}\left(\operatorname{RDef}_{S}\right)$ and $\varphi_{i}$ in $\mathcal{P}_{+}(S[0,0, r])$. Since $f$ is in $\mathrm{I}_{S} \mathscr{C}_{+}(S[0,0, r])$, we may also write $f=\sum_{j} b_{j} \otimes \psi_{j}$, with $b_{j}$ in $S K_{0}\left(\operatorname{RDef}_{S}\right)$ and $\psi_{j}$ in $\mathrm{I}_{S} \mathcal{P}_{+}(S[0,0, r])$. We now use the degree function $\operatorname{deg}_{\mathbb{L}}$ defined in 4.5.5. Recall that, for two functions $\varphi$ and $\varphi^{\prime}$ in $\mathcal{P}_{+}(S[0,0, r]), \operatorname{deg}_{\mathbb{L}}\left(\varphi+\varphi^{\prime}\right)=\sup \left(\operatorname{deg}_{\mathbb{L}}(\varphi), \operatorname{deg}_{\mathbb{L}}\left(\varphi^{\prime}\right)\right)$. Let us also remark that if $a$ belongs to $\mathcal{P}_{+}^{0}(S)$ and $\varphi$ to $\mathcal{P}_{+}(S[0,0, r])$, the difference $\operatorname{deg}_{\mathbb{L}}(a \varphi)-\operatorname{deg}_{\mathbb{L}}\left(\mathbf{1}_{a} \varphi\right)$ may take only a finite number of distinct values, uniformly for $s$ in $|S|$. It then follows from the relations defining the tensor product $S K_{0}\left(\operatorname{RDef}_{S}\right) \otimes_{\mathcal{P}_{+}^{0}(S)} \mathcal{P}_{+}(S[0,0, r])$ that there is a constant $C$ such that $\operatorname{deg}_{\mathbb{L}}\left(\mathbf{1}_{a_{i}} \varphi_{i}\right) \leq C+\sup _{j}\left(\operatorname{deg}_{\mathbb{L}}\left(\psi_{j}\right)\right)$. From Proposition 4.5.6 we deduce that every function $\mathbf{1}_{a_{i}} \varphi_{i}$ is $S$-integrable. The reverse implication being clear, this concludes the proof.

5.8.2. Corollary. - Consider a morphism $g: S \rightarrow \Lambda$ in $\operatorname{Def}_{k}$. For every point $\lambda$ in $\Lambda$ consider the fiber $S_{\lambda}$ of $g$ at $\lambda$. A function $f$ in $\mathscr{C}_{+}(S[0,0, r])$ is $S$-integrable if and only if, for every point $\lambda$ in $\Lambda$, the restriction $f_{\lambda} \in \mathscr{C}_{+}\left(S_{\lambda}[0,0, r]\right)$ of $f$ to

$\overline{{ }^{(6)} \text { The }+ \text { in } \lambda_{+}}$does not refer to positivity but denotes an alternative direct image, which shall only be later on, Proposition 12.1.2, shown to be related with ! of $\lambda_{\text {! }}$. Also it should not be confused with the functor $\lambda_{+}$in $\mathrm{A} 0$ (b) of Theorem 10.1.1. 
$S_{\lambda}[0,0, r]$ is $S$-integrable. Furthermore, if these conditions are satisfied, $\mu_{S_{\lambda}}\left(f_{\lambda}\right)$ is equal to the restriction of $\mu_{S}(f)$ to $S_{\lambda}$.

Proof. - The analogous result with $\mathscr{C}_{+}$replaced by $\mathcal{P}_{+}$being clear, the statement follows directly from Proposition 5.8.1,

5.8.3. Proposition. - Let $S$ be a definable subassignment in GDef $_{k}$. Let $f$ and $g$ be functions in $\mathscr{C}_{+}(S[0,0, r])$. If $f \geq g$ and $f$ is $S$-integrable, then $g$ is $S$-integrable.

Proof. - We may write $f=g+h$ with $h$ in $\mathscr{C}_{+}(S[0,0, r])$. We write $g=\sum_{j} b_{j} \otimes \psi_{j}$, with $b_{j}$ in $S K_{0}\left(\operatorname{RDef}_{S}\right)$ and $\psi_{j}$ in $\mathcal{P}_{+}(S[0,0, r])$, and similarly $h=\sum_{j^{\prime}} b_{j^{\prime}} \otimes \psi_{j^{\prime}}$. Since $f=\sum_{j} b_{j} \otimes \psi_{j}+\sum_{j^{\prime}} b_{j^{\prime}} \otimes \psi_{j^{\prime}}$, it follows from Proposition 5.8.1 that every function $\mathbf{1}_{b_{j}} \psi_{j}$ is $S$-integrable, which concludes the proof.

We now can state the analogue of Lemma 4.5.7 (2). Recall that $h_{\mathbb{Z}^{r}}$ stands for $h[0,0, r]$.

5.8.4. Proposition. - Let $S$ be a definable subassignment in $\operatorname{GDef}_{k}$ and let $\varphi$ be in $\mathscr{C}_{+}\left(S \times h_{\mathbb{Z}^{r}}\right)$. Write $r=r_{1}+r_{2}$ and identify $h_{\mathbb{Z}^{r}}$ with $h_{\mathbb{Z}^{r_{1}}} \times h_{\mathbb{Z}^{r_{2}}}$. The function $\varphi$ is $S$-integrable if and only if, as a function in $\mathscr{C}_{+}\left(S \times h_{\mathbb{Z}^{r_{1}}} \times h_{\mathbb{Z}^{r_{2}}}\right)$, it is $S \times h_{\mathbb{Z}^{r_{1}}}$-integrable and $\mu_{S \times h_{\mathbb{Z}^{r_{1}}}}(\varphi)$ is $S$-integrable.

Proof. - If $\varphi$ is $S \times h_{\mathbb{Z}^{r_{1}}}$-integrable, we may write $\varphi=\sum_{i} a_{i} \otimes \varphi_{i}$ with $a_{i}$ in $S K_{0}\left(\operatorname{RDef}_{S}\right)$ and $\varphi_{i}$ in $\mathrm{I}_{S \times h_{\mathbb{Z}^{r}}} \mathcal{P}_{+}\left(S \times h_{\mathbb{Z}^{r}}\right)$. Replacing $\varphi_{i}$ by $\mathbf{1}_{a_{i}} \varphi_{i}$ we may even assume $\varphi_{i}=\mathbf{1}_{a_{i}} \varphi_{i}$. Hence $\mu_{S \times h_{\mathbb{Z}^{r}}}(\varphi)=\sum_{i} a_{i} \otimes \mu_{S \times h_{\mathbb{Z}^{r}}}\left(\varphi_{i}\right)$. If $\mu_{S \times h_{\mathbb{Z}^{r}}}(\varphi)$ is $S$ integrable, it follows from Proposition 5.8.1 that the functions $\mathbf{1}_{a_{i}} \mu_{S \times h_{\mathbb{Z}^{r}}}\left(\varphi_{i}\right)=$ $\mu_{S \times h_{\mathbb{Z}^{r}}}\left(\varphi_{i}\right)$ are all $S$-integrable. One then deduces from Lemma 4.5.7 (2) that the functions $\varphi_{i}$ are all $S$-integrable, hence $\varphi$ is $S$-integrable. The reverse implication is already known (Proposition 5.7.2).

5.8.5. Proposition. - Let $S$ be a definable subassignment in GDef $_{k}$ and consider the projections $\pi^{1}: S[0, n, r] \rightarrow S[0,0, r]$ and $\pi^{2}: S[0, n, 0] \rightarrow S[0,0,0]$. Let $\varphi$ be a function in $\mathscr{C}_{+}(S[0, n, r])$. Then $\varphi$ is $S[0, n, 0]$-integrable if and only if the function $\pi_{!}^{1}(\varphi)$ in $\mathscr{C}_{+}(S[0,0, r])$ is $S$-integrable. If these conditions hold, then

$$
\pi_{!}^{2}\left(\mu_{S[0, n, 0]}(\varphi)\right)=\mu_{S}\left(\pi_{!}^{1}(\varphi)\right) .
$$

Proof. - Let $\varphi$ be a function in $\mathscr{C}_{+}(S[0, n, r])$. We may write $\varphi=\sum_{i} a_{i} \otimes \varphi_{i}$ with $a_{i}$ in $S K_{0}\left(\operatorname{RDef}_{S[0, n, 0]}\right)$ and $\varphi_{i}$ in $\mathcal{P}_{+}(S[0,0, r])$. Indeed, it follows from the second part of Theorem 2.1.1 that the canonical morphism

$$
\mathcal{P}_{+}^{0}(S[0, n, 0]) \otimes_{\mathcal{P}_{+}^{0}(S)} \mathcal{P}_{+}^{0}(S[0,0, r]) \longrightarrow \mathcal{P}_{+}^{0}(S[0, n, r])
$$

is an isomorphism, from which we deduce a canonical isomorphism

$$
\mathscr{C}_{+}(S[0, n, r]) \simeq S K_{0}\left(\operatorname{RDef}_{S[0, n, 0]}\right) \otimes_{\mathcal{P}_{+}^{0}(S)} \mathcal{P}_{+}(S[0,0, r])
$$

using Propositions 5.2.1 and 5.3.1. We have $\pi_{!}^{1}(\varphi)=\sum_{i} \pi_{!}^{2}\left(a_{i}\right) \otimes \varphi_{i}$. The key remark is that $\mathbf{1}_{a_{i}} \pi^{1 *}\left(\varphi_{i}\right)$ is $S[0, n, 0]$-integrable if and only if $\mathbf{1}_{\pi_{l}^{2} a_{i}} \varphi_{i}$ is $S$-integrable, which holds since integrability is defined by a pointwise condition. Hence, it 
follows from Proposition 5.8.1 that $\varphi$ is $S[0, n, 0]$-integrable if and only if $\pi_{!}^{1}(\varphi)$ is $S$-integrable. Let us assume that these conditions hold, so that, by Proposition 5.8.1, each $\mathbf{1}_{a_{i}} \varphi_{i}$ is $S$-integrable. We assume $\varphi_{i}=\mathbf{1}_{a_{i}} \varphi_{i}$ for every $i$. Since $\mu_{S[0, n, 0]}\left(\pi^{1 *}\left(\varphi_{i}\right)\right)=\pi^{2 *}\left(\mu_{S}\left(\varphi_{i}\right)\right)$, we get $\mu_{S[0, n, 0]}(\varphi)=\sum_{i} a_{i} \otimes \mu_{S}\left(\varphi_{i}\right)$, and hence, we deduce $\pi_{!}^{2} \mu_{S[0, n, 0]}(\varphi)=\sum_{i} \pi_{!}^{2}\left(a_{i}\right) \otimes \mu_{S}\left(\varphi_{i}\right)=\mu_{S}\left(\pi_{!}^{1}(\varphi)\right)$.

Let $\lambda: S[0, n, r] \rightarrow S\left[0, n^{\prime}, r^{\prime}\right]$ be a morphism in $\operatorname{Def}_{S}$. Let $\varphi$ be a function in $\mathscr{C}(S[0, n, r])$, resp. in $\mathscr{C}_{+}(S[0, n, r])$. Assume $\varphi=\mathbf{1}_{Z} \varphi$ with $Z$ a definable subassignment of $S[0, n, r]$ on which $\lambda$ is injective. Thus $\lambda$ restricts to an isomorphism $\lambda^{\prime}$ between $Z$ and $Z^{\prime}:=\lambda(Z)$. We define $\lambda_{+}(\varphi)$ in $\mathscr{C}\left(S\left[0, n^{\prime}, r^{\prime}\right]\right)$, resp. in $\mathscr{C}_{+}\left(S\left[0, n^{\prime}, r^{\prime}\right]\right)$ as $\left[i_{!}^{\prime}\left(\lambda^{\prime-1}\right)^{*} i^{*}\right](\varphi)$, where $i$ and $i^{\prime}$ denote respectively the inclusions of $Z$ and $Z^{\prime}$ in $S[0, n, r]$ and $S\left[0, n^{\prime}, r^{\prime}\right]$. Clearly this definition does not depend on the choice of $Z$ and is compatible with the definition of $\lambda_{+}$in section 5.7 when $n=n^{\prime}=0$.

5.8.6. Proposition. - Let $\lambda: S[0, n, r] \rightarrow S\left[0, n^{\prime}, r^{\prime}\right]$ be a morphism in $\operatorname{Def}_{S}$. Let $\varphi$ be a function in $\mathscr{C}_{+}(S[0, n, r])$ such that $\varphi=\mathbf{1}_{Z} \varphi$ with $Z$ a definable subassignment of $S[0, n, r]$ on which $\lambda$ is injective. Then $\varphi$ is $S[0, n, 0]$-integrable if and only if $\lambda_{+}(\varphi)$ is $S\left[0, n^{\prime}, 0\right]$-integrable and if this is the case then

$$
p_{!}\left(\mu_{S[0, n, 0]}(\varphi)\right)=p_{!}^{\prime}\left(\mu_{S\left[0, n^{\prime}, 0\right]}\left(\lambda_{+}(\varphi)\right)\right),
$$

with $p: S[0, n, 0] \rightarrow S$ and $p^{\prime}: S\left[0, n^{\prime}, 0\right] \rightarrow S$ the projections.

Proof. - In the case where $n=n^{\prime}$ and $\lambda$ is the identity on the $\mathbb{A}_{k}^{n}$-factor, that is, $\lambda$ is of the form $(s, \xi, \alpha) \mapsto(s, \xi, g(s, \alpha))$, with $s$ variable on the $S$-factor, $\xi$ on the $\mathbb{A}_{k}^{n}$-factor and $\alpha$ on the $\mathbb{Z}^{r}$-factor, the statement follows directly from Proposition 5.7.3. Assume now that $r=r^{\prime}$ and that $\lambda$ is the identity on the $\mathbb{Z}^{r}$-factor, that is, $\lambda$ is of the form $(s, \xi, \alpha) \mapsto(s, f(s, \xi), \alpha)$. Write $g: S[0, n, 0] \rightarrow S\left[0, n^{\prime}, 0\right]:$ $(s, \xi) \mapsto(s, f(s, \xi))$. Let $\varphi$ be a function in $\mathscr{C}_{+}(S[0, n, r])$ such that $\varphi=\mathbf{1}_{Z} \varphi$ with $Z$ a definable subassignment of $S[0, n, r]$ on which $\lambda$ is injective. As in the proof of Proposition 5.8.5 we may write

$$
\varphi=\sum_{i} a_{i} \otimes \varphi_{i}
$$

with $a_{i}$ in $S K_{0}\left(\operatorname{RDef}_{S[0, n, 0]}\right), \varphi_{i}$ in $\mathcal{P}_{+}(S[0,0, r])$. By the second part of Theorem 2.1.1, we may assume that $g$ is injective on $\operatorname{Supp}\left(a_{i}\right)$ for each $i$. Using the injectivity of $g$ on $\operatorname{Supp}\left(a_{i}\right)$, we can write

$$
\lambda_{+}(\varphi)=\sum_{i} g_{!}\left(a_{i}\right) \otimes \varphi_{i}
$$

where $g_{!}\left(a_{i}\right)$ in $S K_{0}\left(\operatorname{RDef}_{S\left[0, n^{\prime}, 0\right]}\right)$ is defined as $\left[X_{i} \otimes_{S\left[0, n^{\prime}, 0\right]} S\left[0, n^{\prime}, 0\right] \rightarrow S\left[0, n^{\prime}, 0\right]\right]$ with $a_{i}=\left[X_{i} \rightarrow S[0, n, 0]\right]^{(7)}$. If $\varphi$ is $S[0, n, 0]$-integrable, we may assume all the functions $\varphi_{i}$ are $S$-integrable, hence $\lambda_{+}(\varphi)$ is $S\left[0, n^{\prime}, 0\right]$-integrable. For the reverse implication, note that $\varphi=\tilde{\lambda}_{+}\left(\lambda_{+}(\varphi)\right)$ for any morphism $\tilde{\lambda}: S\left[0, n^{\prime}, r^{\prime}\right] \rightarrow S[0, n, r]$

(7) This definition of $g_{\text {! }}$ is consistent with the definition of the push-forward in section [5.6. Note that $a_{i}$ can always be written in the form $\left[X_{i} \rightarrow S[0, n, 0]\right]$. 
which restricts to the inverse of $\lambda^{\prime}$ on $Z^{\prime}$. Relation (5.8.1) then follows from (5.8.2), (5.8.3), and from the obvious relation $p_{!}=p_{!}^{\prime} \circ g_{!}$.

Note that if the statement of Proposition 5.8.6 holds for two composable morphisms $\lambda_{1}$ and $\lambda_{2}$, it still holds for $\lambda_{2} \circ \lambda_{1}$. In particular it follows from the previous discussion that the statement we want to prove holds for $\lambda$ of the form $(s, \xi, \alpha) \mapsto(s, f(s, \xi), g(s, \alpha))$. Now consider the case of a general morphism $\lambda$ : $S[0, n, r] \rightarrow S\left[0, n^{\prime}, r^{\prime}\right]$. Let $\varphi$ be a function in $\mathscr{C}_{+}(S[0, n, r])$ such that $\varphi=\mathbf{1}_{Z} \varphi$ with $Z$ a definable subassignment of $S[0, n, r]$ on which $\lambda$ is injective. By the second part of Theorem 2.1.1 there is a finite partition of $Z$ into definable subassignments $Z_{i}$, such that the restriction of $\lambda$ to each $Z_{i}$ is of the form $(s, \xi, \alpha) \mapsto\left(s, f_{i}(s, \xi), g_{i}(s, \alpha)\right)$. Since $\lambda_{+}\left(\mathbf{1}_{Z_{i}} \varphi\right)$ only depends on the restriction of $\lambda$ to $Z_{i}$, it follows that the statement we want to prove holds for $\mathbf{1}_{Z_{i}} \varphi$, hence also for $\varphi=\sum_{i} \mathbf{1}_{Z_{i}} \varphi$.

\section{Constructible motivic Functions}

6.1. Dimension and relative dimension. - Let $Z$ be in $\operatorname{Def}_{k}$ and let $\varphi$ be in $\mathscr{C}(Z)$, resp. $\mathscr{C}_{+}(Z)$. We say $\varphi$ is of $K$-dimension $\leq d$ if it may be written as a finite sum $\varphi=\sum \lambda_{i} \mathbf{1}_{Z_{i}}$ in $\mathscr{C}(Z)$, resp. in $\mathscr{C}_{+}(Z)$, with $\operatorname{Kdim} Z_{i} \leq d$. We say $\varphi$ is of $K$-dimension $d$ if it is of $K$-dimension $\leq d$ and not of $K$-dimension $\leq d-1$.

More generally, if $Z \rightarrow S$ is in $\operatorname{Def}_{S}$ for some $S$ and $\varphi$ in $\mathscr{C}(Z)$, resp. in $\mathscr{C}_{+}(Z)$, we say that $\varphi$ is of dimension $\leq d$ rel. the projection $Z \rightarrow S$ if it may be written as a finite $\operatorname{sum} \varphi=\sum \lambda_{i} \mathbf{1}_{Z_{i}}$ in $\mathscr{C}(Z)$, resp. in $\mathscr{C}_{+}(Z)$, with $Z_{i}$ of relative dimension $\leq d$ rel. the projection $Z \rightarrow S$ (as in section 3.4). We also use the notion of dimension $d$ rel. the projection $Z \rightarrow S$, for $\varphi$ in $\mathscr{C}(Z)$ or in $\mathscr{C}_{+}(Z)$ if it is of relative dimension $\leq d$ but not of relative dimension $\leq d-1$

6.2. Constructible motivic Functions. - Let $Z$ be a definable subassignment in $\operatorname{GDef}_{k}$. We denote by $\mathscr{C}^{\leq d}(Z)$, resp. $\mathscr{C}_{+}^{\leq d}(Z)$, the subgroup, resp. subsemigroup, of elements of $\mathscr{C}(Z)$, resp. $\mathscr{C}_{+}(Z)$, of $K$-dimension $\leq d$. We denote by $C^{d}(Z)$ the quotient

$$
C^{d}(Z):=\mathscr{C}^{\leq d}(Z) / \mathscr{C}^{\leq d-1}(Z)
$$

and we set

$$
C(Z)=\bigoplus_{d \geq 0} C^{d}(Z)
$$

which is a graded abelian group. Similarly, we denote by $C_{+}^{d}(Z)$ the quotient

$$
C_{+}^{d}(Z):=\mathscr{C}_{+}^{\leq d}(Z) / \mathscr{C}_{+}^{\leq d-1}(Z)
$$

and we consider the graded abelian semigroup

$$
C_{+}(Z)=\bigoplus_{d \geq 0} C_{+}^{d}(Z)
$$

An element in $C(Z)$, resp. in $C_{+}(Z)$, will be called a constructible motivic Function, resp. a positive constructible motivic Function (note the capital $F$ ). It is an 
equivalence class of constructible motivic functions, resp. of positive constructible motivic functions.

If $\varphi$ in $\mathscr{C}(Z)$ or in $\mathscr{C}_{+}(Z)$ is of $K$-dimension $d$, or if $\varphi=0$ in $\mathscr{C}(Z)$, we denote by $[\varphi]$ the class of $\varphi$ in $C^{d}(Z)$ and in $C_{+}^{d}(Z)$.

Let us remark that, since $\mathscr{C}^{\leq d}(Z)$ is an ideal in $\mathscr{C}(Z)$, the product on $\mathscr{C}(Z)$ induces a $\mathscr{C}(Z)$-module structure on $C(Z)$ and on each $C^{d}(Z)$. Similarly the product on $\mathscr{C}_{+}(Z)$ induces a $\mathscr{C}_{+}(Z)$-module structure on $C_{+}(Z)$.

6.3. - More generally, let us fix a definable subassignment $S$ in GDef $_{k}$ and consider the category $\operatorname{GDef}_{S}$. For $Z$ in $\operatorname{GDef}_{S}$, we define $\mathscr{C}^{\leq d}(Z \rightarrow S)$ and $\mathscr{C}_{+}^{\leq d}(Z \rightarrow S)$ as in 6.2, but by replacing $K$-dimension by relative $K$-dimension relative to $S$.

We set $C^{d}(Z \rightarrow S):=\mathscr{C}^{\leq d}(Z \rightarrow S) / \mathscr{C}^{\leq d-1}(Z \rightarrow S)$ and

$$
C(Z \rightarrow S)=\bigoplus_{d \geq 0} C^{d}(Z \rightarrow S)
$$

One defines similarly $C_{+}^{d}(Z \rightarrow S)$ and $C_{+}(Z \rightarrow S)$. Also, if $\varphi$ is of relative dimension $d$, or if $\varphi=0$ in $\mathscr{C}(Z \rightarrow S)$, we denote by $[\varphi]$ the class of $\varphi$ in $C^{d}(Z \rightarrow S)$ and in $C_{+}^{d}(Z \rightarrow S)$. Let us remark that $C($ id $: S \rightarrow S)=\mathscr{C}(S)$ and that $C\left(Z \rightarrow h_{\text {Spec } k}\right)=$ $C(Z)$ and similarly for $C_{+}$.

Let $f$ be a Function in $C(Z \rightarrow S)$. For every point $s$ in $S, f$ naturally restricts to a Function $f_{s}$ in $C\left(Z_{s}\right)$, where $Z_{s}$ denotes the fiber of $Z$ at $s$.

If $Z_{1}$ and $Z_{2}$ are disjoint definable subassignment of some definable subassignment in $\operatorname{GDef}_{S}$, then (5.4.1) induces isomorphisms

$$
C\left(Z_{1} \cup Z_{2} \rightarrow S\right) \simeq C\left(Z_{1} \rightarrow S\right) \oplus C\left(Z_{2} \rightarrow S\right)
$$

and

$$
C_{+}\left(Z_{1} \cup Z_{2} \rightarrow S\right) \simeq C_{+}\left(Z_{1} \rightarrow S\right) \oplus C_{+}\left(Z_{2} \rightarrow S\right)
$$

Also, (5.4.2) induces morphisms

$$
C\left(Z_{1} \rightarrow S\right) \otimes_{\mathscr{C}(S)} C\left(Z_{2} \rightarrow S\right) \rightarrow C\left(Z_{1} \times{ }_{S} Z_{2} \rightarrow S\right)
$$

and

$$
C_{+}\left(Z_{1} \rightarrow S\right) \otimes_{\mathscr{C}_{+}(S)} C_{+}\left(Z_{2} \rightarrow S\right) \rightarrow C_{+}\left(Z_{1} \times{ }_{S} Z_{2} \rightarrow S\right)
$$

\section{Cell decomposition}

In this section we shall state some variants and mild generalizations of the Cell Decomposition Theorem of [30] in a form suitable for our needs. Our terminology concerning cells differs slightly from that used in [30]. 
7.1. Cells. - Let $S$ be in $\operatorname{Def}_{k}$. We will define the notion of a cell $Z \subset S[1,0,0]$, at first with a base $C \subset S$, and secondly with a more general base $C \subset S[0, r, s]$.

First let $C$ be a definable subassigment of $S$. Let $\alpha, \xi$, and $c$ be definable morphisms $\alpha: C \rightarrow \mathbb{Z}, \xi: C \rightarrow h_{\mathbb{G}_{m, k}}$, and $c: C \rightarrow h[1,0,0]$. The 1-cell $Z_{C, \alpha, \xi, c}$ with basis $C$, order $\alpha$, center $c$, and angular component $\xi$ is the definable subassignment of $S[1,0,0]$ defined by $y$ in $C$, ord $(z-c(y))=\alpha(y)$, and $\overline{\mathrm{ac}}(z-c(y))=\xi(y)$, where $y$ lies in $S$ and $z$ in $h[1,0,0]$. Similarly, if $c$ is a definable morphism $c: C \rightarrow h[1,0,0]$, we define the 0 -cell $Z_{C, c}$ with center $c$ and basis $C$ as the definable subassignment of $S[1,0,0]$ defined by $y \in C$ and $z=c(y)$.

Secondly and finally, a definable subassignment $Z$ of $S[1,0,0]$ will be called a 1-cell, resp. a 0-cell, if there exists a definable isomorphism

$$
\lambda: Z \rightarrow Z_{C}=Z_{C, \alpha, \xi, c} \subset S[1, s, r],
$$

resp. a definable isomorphism

$$
\lambda: Z \rightarrow Z_{C}=Z_{C, c} \subset S[1, s, 0],
$$

for some $s, r \geq 0$, some basis $C \subset S[0, s, r]$, resp. $C \subset S[0, s, 0]$, and some 1-cell $Z_{C, \alpha, \xi, c}$, resp. 0-cell $Z_{C, c}$, such that the morphism $\pi \circ \lambda$, with $\pi$ the projection on the $S[1,0,0]$-factor, is the identity on $Z$.

We shall call the data $\left(\lambda, Z_{C, \alpha, \xi, c}\right)$, resp. $\left(\lambda, Z_{C, c}\right)$, sometimes written for short $\left(\lambda, Z_{C}\right)$, a presentation of the cell $Z$.

One should note that $\lambda^{*}$ induces a canonical bijection between $\mathscr{C}\left(Z_{C}\right)$ and $\mathscr{C}(Z)$.

7.1.1. Remark. - Cells as defined in 30] fall within our definition, but not the other way around. Also, any presentation as in 30 of a cell as in 30 can be adapted to a presentation in the above sense; for a 0-cell this is trivial, and for a 1-cell this can be done by adding one more Ord-variable.

7.2. Cell decomposition. - The following variant of the Denef-Pas Cell Decomposition Theorem [30] will play a fundamental role in the present paper:

7.2.1. Theorem. - Let $X$ be a definable subassignment of $S[1,0,0]$ with $S$ in $\operatorname{Def}_{k}$.

(1) The subassigment $X$ is a finite disjoint union of cells.

(2) For every $\varphi$ in $\mathscr{C}(X)$ there exists a finite partition of $X$ into cells $Z_{i}$ with presentation $\left(\lambda_{i}, Z_{C_{i}}\right)$, such that $\varphi_{\mid Z_{i}}=\lambda_{i}^{*} p_{i}^{*}\left(\psi_{i}\right)$, with $\psi_{i}$ in $\mathscr{C}\left(C_{i}\right)$ and $p_{i}$ : $Z_{C_{i}} \rightarrow C_{i}$ the projection. Similar statements hold for $\varphi$ in $\mathscr{C}_{+}(X)$, in $\mathcal{P}(X)$, in $\mathcal{P}_{+}(X)$, in $K_{0}\left(\operatorname{RDef}_{X}\right)$, and in $S K_{0}\left(\operatorname{RDef}_{X}\right)$.

We shall call a finite partition of $X$ into cells $Z_{i}$ as in Theorem 7.2.1(1), resp. 7.2.1(2) for a function $\varphi$, a cell decomposition of $X$, resp. a cell decomposition of $X$ adapted to $\varphi$.

Proof of Theorem 7.2.1. - Clearly (2) implies (1). We show how (2) follows from the cell decomposition Theorem. 3.2 of [30]. To fix notation, let $S$ be a definable subassignment of $h[m, n, r]$. 
First we let $\varphi$ be in $\mathscr{C}(X)$. Write $\varphi$ as $\sum_{i} a_{i} \otimes \varphi_{i}$ with $a_{i}$ in $K_{0}\left(\operatorname{RDef}_{X}\right)$ and $\varphi_{i}$ in $\mathcal{P}(X)$. Let $f_{1}, \ldots, f_{t}$ be all the polynomials in the Val-variables occurring in the formulas describing the data $X, a_{i}$, and $\varphi_{i}$, where we may suppose that these formulas do not contain quantifiers over the valued field sort. Apply the cell decomposition Theorem 3.2 of 30 to the polynomials $f_{i}$. Using Remark 7.1.1, we see that this yields a partition of $h[m+1,0,0]$ into cells $\tilde{Z}_{i}$ with presentations $\lambda_{i}: \tilde{Z}_{i} \rightarrow \tilde{Z}_{C_{i}}$ and with some center $c_{i}$. Write $x=\left(x_{1}, \ldots, x_{m+1}\right)$ for the Valvariables, $\xi=\left(\xi_{i}\right)$ for the Res-variables and $z=\left(z_{i}\right)$ for the Ord-variables on $\tilde{Z}_{C_{i}}$.

If $\tilde{Z}_{i}$ is a 1-cell, we may suppose that for $(x, \xi, z)$ in $\tilde{Z}_{C_{i}}$ we have $\operatorname{ord}\left(x_{m+1}-c_{i}\right)=z_{1}$ and $\overline{\mathrm{ac}}\left(x_{m+1}-c_{i}\right)=\xi_{1}$, by changing the presentation of $\tilde{Z}_{i}$ if necessary (that is, by adding more Ord-variables and Res-variables). By the application of Theorem 3.2 of [30] and by changing the presentation as before if necessary, we may also assume that

$$
\begin{aligned}
& \operatorname{ord} f_{j}(x)=z_{k_{j}}, \\
& \overline{\operatorname{ac}} f_{j}(x)=\xi_{l_{j}},
\end{aligned}
$$

for $(x, \xi, z)$ in a 1-cell $\tilde{Z}_{C_{i}}$, where the indices $k_{j}$ and $l_{j}$ only depend on $j$ and $i$.

Since the condition $f(x)=0$ is equivalent to $\overline{\mathrm{ac}}(f(x))=0$, we may suppose that, in the formulas describing $X, a_{i}$, and $\varphi_{i}$, the only terms involving Val-variables are of the forms ord $f_{j}(x)$ and $\overline{\mathrm{ac}} f_{j}(x)$. Combining this with the above description of ord $f_{j}(x)$ and $\overline{\mathrm{ac}} f_{j}(x)$ one can then easily construct a partition of $X$ into cells $Z_{i}$ and for each such cell a constructible functions $\psi$ which satisfies the requirements of the theorem. If $\varphi$ is in $\mathscr{C}_{+}(X)$, resp. in $\mathcal{P}(X), \mathcal{P}_{+}(X), K_{0}\left(\operatorname{RDef}_{X}\right)$, or in $S K_{0}\left(\operatorname{RDef}_{X}\right)$, the same argument works.

7.2.2. Example. - A cell decomposition for $h_{\mathbb{A}_{k(t))}^{1}}$ : take the disjoint union of the 0-cell $\{0\}$ and the 1-cell $h_{\mathbb{A}_{k((t))}^{1}} \backslash\{0\}$ with presentation $\left(\lambda, Z^{\prime}\right)$ with $\lambda(z)=$ $(z, \overline{\operatorname{ac}}(z), \operatorname{ord}(z))$ and $Z^{\prime}$ defined by $\overline{\operatorname{ac}}(z)=\xi, \operatorname{ord}(z)=i, \xi \neq 0$.

7.2.3. Corollary. - Let $Y$ and $Z \subset Y\left[m^{\prime}, 0,0\right]$ be definable subassignments and let $f: Z \rightarrow Y$ be the projection. Suppose that for each $y=\left(y_{0}, K\right)$ in $Y$, the set $Z_{y}(K)$ is finite, with $Z_{y}$ the fiber above $y$. Then the cardinality of $Z_{y}(K)$ is bounded uniformly in $y=\left(y_{0}, K\right)$, and, there exists a definable isomorphism $g: Z \rightarrow Y^{\prime} \subset$ $Y[0, n, r]$ over $Y$ such that $p \circ g=f$, with $p: Y[0, n, r] \rightarrow Y$ the projection.

Proof. - Replacing $Z$ by the graph of $f$, the essential case to prove is when $Z \subset$ $h\left[m+m^{\prime}, n, r\right], Y=h[m, n, r]$, and $f$ is the projection. First suppose $m^{\prime}=1$. Applying Theorem 7.2.1 to $Z$, the proposition follows immediately piecewise, and hence globally, since a finite partition can be replaced by one part by allowing for extra parameters. The case of $m^{\prime}>1$ is treated by an inductive application of Theorem 7.2.1,

${ }^{(8)} \mathrm{By}$ this we mean that we take the defining formulas for $X$ and, for each $i$, of subassignments in $\operatorname{RDef}_{X}$ representing $a_{i}$ and the defining formulas for all definable morphisms $\alpha: X \rightarrow \mathbb{Z}$ occurring in $\varphi_{i}$, written as a sum of products of constants in $\mathbb{A}$ and functions of the forms $\mathbb{L}^{\alpha}$ and $\alpha$. 
7.2.4. Corollary. - Let $S$ be in $\operatorname{Def}_{k}$ and $f: S[1,0,0] \rightarrow h[1,0,0]$ be a definable morphism. Then there exists a definable isomorphism $g: S[1,0,0] \rightarrow Z \subset S[1, n, r]$ over $S[1,0,0]$ and a partition of $Z$ into parts $X, Y$ such that for each $s=\left(s_{0}, K\right)$ in $S[0, n, r]$ the map $X_{s}(K) \rightarrow K((t)): u \mapsto f \circ g^{-1}(s, u)$ is injective and the map $Y_{s}(K) \rightarrow K((t)): u \mapsto f \circ g^{-1}(s, u)$ is constant, with $X_{s}$ and $Y_{s}$ the fiber of $X$, resp. of $Y$, above $s$ under the projection $X \rightarrow S[0, n, r]$, resp. $Y \rightarrow S[0, n, r]$.

Proof. - It follows from Theorem 3.2.1, the definition of the dimension of definable subassignments, and the Implicit Function Theorem for $K((t))$-analytic maps, that there is a partition $\left\{X_{0}, Y_{0}\right\}$ of $S[1,0,0]$ such that for each $s=\left(s_{0}, K\right)$ in $S$ the map $X_{0 s}(K) \rightarrow K((t)): u \mapsto f(s, u)$ is finite to one and the map $Y_{0 s}(K) \rightarrow K((t))$ : $u \mapsto f(s, u)$ has finite image, with $X_{0 s}$ and $Y_{0 s}$ the fibers. To obtain $X$, apply Corollary 7.2 .3 to the map $X_{0} \rightarrow S[1,0,0]:(s, u) \mapsto(s, f(s, u))$. To obtain $Y$, apply Corollary 7.2 .3 to the projection map $\pi: p\left(\operatorname{Graph}\left(f_{\mid Y_{0}}\right)\right) \rightarrow S$ where $p$ is the projection $S[2,0,0] \rightarrow S[1,0,0]$ onto the $(s, f)$ variables.

7.2.5. Lemma. - Let $\varphi$ be in $\mathscr{C}_{+}(S[1,0,0])$ for some $S$ in $\operatorname{Def}_{k}$ and let $Z \subset$ $S[1,0,0]$ be a 1-cell which is adapted to $\left.\varphi\right|_{Z}$ and which has a presentation $\lambda: Z \rightarrow$ $Z_{C}=Z_{C, \alpha, \xi, c}$. There is some $\psi$ in $\mathscr{C}_{+}(C)$ with $\lambda^{*} p^{*}(\psi)=\left.\varphi\right|_{Z}$, where $p: Z_{C} \rightarrow C$ is the projection. Let $C_{G}$ be any nonempty definable subassignment of $C$ and let $\alpha_{G}, \xi_{G}, c_{G}$ be the restrictions of $\alpha, \xi, c$ to $C_{G}$. Then, the subassignment $Z_{G}$ of $Z$ given by $p \circ \lambda(x) \in C_{G}$ is a 1-cell with presentation $\lambda_{G}: Z_{G} \rightarrow Z_{C_{G}, \alpha_{G}, \xi_{G}, c_{G}}$, where $\lambda_{G}$ is the restriction of $\lambda$ to $Z_{G}$. Moreover, $Z_{G}$ is adapted to $\left.\varphi\right|_{Z_{G}}$ and $\left.\varphi\right|_{Z_{G}}=\lambda_{G}^{*} p_{G}^{*}\left(\psi_{G}\right)$, where $\psi_{G}$ is the restriction of $\psi$ to $C_{G}$ and $p_{G}$ the restriction of $p$ to $Z_{G}$. A similar statement holds for 0 -cells.

Proof. - Clear.

7.3. Refinements. - Let $\mathcal{P}=\left(Z_{i}\right)_{i \in I}$ and $\mathcal{P}^{\prime}=\left(Z_{j}^{\prime}\right)_{j \in J}$ be two cell decompositions of a definable subassignment $X \subset S[1,0,0]$ for some $S$ in $\operatorname{Def}_{k}$. We say $\mathcal{P}$ is a refinement of $\mathcal{P}^{\prime}$ and write $\mathcal{P} \prec \mathcal{P}^{\prime}$ if for every $j$ in $J$ there exists $i(j)$ in $I$ such that $Z_{i(j)} \subset Z_{j}^{\prime}$.

7.3.1. Lemma. - Let $Z$ and $Z^{\prime}$ be cells in $S[1,0,0]$ for some $S$ in $\operatorname{Def}_{k}$. Let $\left(\lambda^{\prime}, Z_{C^{\prime}}^{\prime}\right)$ be a presentation of $Z^{\prime}$. If $Z \subset Z^{\prime}$ then there exists a presentation $\left(\lambda, Z_{C}\right)$ of $Z$ and a (necessarily unique) definable morphism $g: C \rightarrow C^{\prime}$ such that $g \circ p \circ \lambda=$ $p^{\prime} \circ \lambda_{\mid Z}^{\prime}$, where $p: Z_{C} \rightarrow C$ and $p^{\prime}: Z_{C^{\prime}}^{\prime} \rightarrow C^{\prime}$ are the projections.

Proof. - If $Z$ is a 0-cell the statement is clear. Now suppose that $Z$ is a 1-cell with some presentation $\left(\lambda_{0}, Z_{C_{0}}\right)$. Then $Z^{\prime}$ is also a 1-cell with some presentation $\left(\lambda^{\prime}, Z_{C^{\prime}}^{\prime}\right)$. Let $\lambda$ be the presentation of $Z$ given by

$$
Z \rightarrow Z_{C_{0} \times C^{\prime}}: z \mapsto\left(\lambda_{0}(z), p^{\prime} \circ \lambda^{\prime}(z)\right) .
$$

By the non archimedean property this is indeed a presentation of the 1-cell $Z$, and the uniqueness and existence of $g$ for this $\lambda$ is clear. 
7.3.2. Proposition. - Let $X$ be a definable subassignment of $S[1,0,0]$ with $S$ in $\operatorname{Def}_{k}$ and let $\varphi_{i}$ be a function in $\mathscr{C}(X)$ for $i=1,2$. Let $\mathcal{P}_{i}$ be a cell decomposition of $X$ adapted to $\varphi_{i}$ for $i=1,2$. Then there exists a cell decomposition $\mathcal{P}$ of $X$ such that $\mathcal{P} \prec \mathcal{P}_{i}$ for $i=1,2$. Such $\mathcal{P}$ is automatically adapted to both $\varphi_{1}$ and $\varphi_{2}$. Similar statements hold for $\varphi_{i}$ in $\mathscr{C}_{+}(X)$, in $\mathcal{P}(X)$, in $\mathcal{P}_{+}(X)$, in $K_{0}\left(\operatorname{RDef}_{X}\right)$, and in $S K_{0}\left(\operatorname{RDef}_{X}\right)$.

Proof. - It follows from Lemma 7.3.1 that if $\mathcal{P}$ is a refinement of $\mathcal{P}_{1}$ then $\mathcal{P}$ is automatically adapted to $\varphi_{1}$. Thus we only have to show that there is a common refinement $\mathcal{P}$ of $\mathcal{P}_{1}$ and $\mathcal{P}_{2}$, but this follows at once from the definitions and Theorem 7.2.1.

7.4. Bicells. - We will have, for technical reasons, to consider bicells, that is, cells with 2 special variables. More precisely, they will be needed for a basic version of Fubini's Theorem.

Fix $S$ in $\operatorname{Def}_{k}$. As for the definition of cells in [7.1, we shall first define bicells $Z \subset S[2,0,0]$ with base $C \subset S$, and then with more general base $C \subset S[0, s, i]$. Let first $C$ be a definable subassigment of $S$. Let $\alpha$ and $\beta$ be definable morphisms $C \rightarrow \mathbb{Z}$, $\xi$ and $\eta$ definable morphisms $C \rightarrow h_{\mathbb{G}_{m, k}}, c$ a definable morphism $C \rightarrow h[1,0,0]$, and let $d$ be a definable morphism $C[1,0,0] \rightarrow h[1,0,0]$. We further assume that either, for every point $y=\left(y_{0}, K\right)$ in $C$, the function $u \mapsto d(y, u)$ is constant on $K((t))$, or, for every point $y=\left(y_{0}, K\right)$ in $C$, it is injective on $K((t))$.

The bicell $Z_{C, \alpha, \beta, \xi, \eta, c, d}$ with basis $C$ is the definable subassignment of $S[2,0,0]$ defined by

$$
\begin{gathered}
y \in C \\
\operatorname{ord}(z-d(y, u))=\alpha(y) \\
\overline{\operatorname{ac}}(z-d(y, u))=\xi(y) \\
\operatorname{ord}(u-c(y))=\beta(y) \\
\overline{\operatorname{ac}}(u-c(y))=\eta(y),
\end{gathered}
$$

where $y$ denotes the $S$-variable, $z$ the first $\mathbb{A}_{k((t))}^{1}$-variable and $u$ the second $\mathbb{A}_{k(t))^{-}}^{1}$ variable.

Similarly, we define the bicell $Z_{C, \beta, \eta, c, d}^{\prime}$ as the definable subassignment of $S[2,0,0]$ defined by

$$
\begin{gathered}
y \in C \\
z=d(y, u) \\
\operatorname{ord}(u-c(y))=\beta(y) \\
\overline{\operatorname{ac}}(u-c(y))=\eta(y),
\end{gathered}
$$


the bicell $Z_{C, \alpha, \xi, c, d}^{\prime \prime}$ as the definable subassignment defined by

$$
\begin{gathered}
y \in C \\
\operatorname{ord}(z-d(y, u))=\alpha(y) \\
\overline{\operatorname{ac}}(z-d(y, u))=\xi(y) \\
u=c(y),
\end{gathered}
$$

and the bicell $Z_{C, c, d}^{\prime \prime \prime}$ as the definable subassignment defined by

$$
\begin{gathered}
y \in C \\
z=d(y, u) \\
u=c(y) .
\end{gathered}
$$

Now we can define bicells similarly to 7.1 with general base $C \subset S[0, s, i]$, except that we have 4 types of bicells instead of 2 types of cells. A definable subassignment $Z$ of $S[2,0,0]$ will be called a (1,1)-bicell (resp. a (1,0)-bicell, a $(0,1)$-bicell, a $(0,0)$ bicell), if there exists a definable isomorphism

$$
\lambda: Z \longrightarrow Z_{C} \subset S[2, s, i]
$$

and a bicell $Z_{C}=Z_{C, \alpha, \beta, \xi, \eta, c, d}\left(\operatorname{resp} . Z_{C}=Z_{C, \beta, \eta, c, d}^{\prime}, Z_{C}=Z_{C, \alpha, \xi, c, d}^{\prime \prime}, Z_{C}=Z_{C, c, d}^{\prime \prime \prime}\right)$ with basis $C \subset S[0, s, i]$, such that the morphism $\pi \circ \lambda$, with $\pi$ the projection on the $S[2,0,0]$-factor, restricts to the identity on $Z$.

We shall call the data $\left(\lambda, Z_{C}\right)$ with $Z_{C}$ of one of the above forms a presentation of the cell $Z$.

We define similarly to 7.2 bicell decompositions of a definable subassignment $Z$ of $S[2,0,0]$ and bicell decompositions of $Z$ adapted to a given function $\varphi$ in $\mathscr{C}(Z)$.

The following statement is an easy consequence of Theorem 7.2.1 and its Corollary 7.2.4;

7.4.1. Proposition. — (1) Every definable subassignment $Z$ of $S[2,0,0]$ admits a bicell decomposition.

(2) For every $\varphi$ in $\mathscr{C}(Z)$ there exists a bicell decomposition of $Z$ adapted to the function $\varphi$, namely, there exists a finite partition of $Z$ into bicells $Z_{i}$ with presentation $\left(\lambda_{i}, Z_{C_{i}}\right)$, such that $\varphi_{\mid Z_{i}}=\lambda_{i}^{*} p_{i}^{*}\left(\psi_{i}\right)$, with $\psi_{i}$ in $\mathscr{C}\left(C_{i}\right)$ and $p_{i}$ : $Z_{C_{i}} \rightarrow C_{i}$ the projection. Similar statements hold for $\varphi$ in $\mathscr{C}_{+}(Z)$, in $\mathcal{P}(Z)$, in $\mathcal{P}_{+}(Z)$, in $K_{0}\left(\operatorname{RDef}_{Z}\right)$, and in $S K_{0}\left(\operatorname{RDef}_{Z}\right)$.

Proof. - First apply Theorem 7.2.1 to obtain a partition of $Z$ into cells, adapted to $\varphi$. We apply now Corollary 7.2.4 to each center to partition each basis. By Lemma 7.2.5 this yields a partition of $Z$ into cells, refining the previous partition. We finish the proof by applying Theorem 7.2.1 to each basis $C$ of the occurring cells and the functions $\psi$ in $\mathscr{C}(C)$ corresponding to $\varphi$ as in Theorem 7.2 .1 (2). 
7.5. Analyticity and cell decomposition. - We consider the expansion $\mathcal{L}_{\mathrm{DP}}^{*}$ of $\mathcal{L}_{\mathrm{DP}}$ which is obtained by adding the following function symbols for each integer $n>0$ :

(1) The symbol ${ }^{-1}:$ Val $\rightarrow$ Val for the field inverse on Val $\backslash\{0\}$ extended by $0^{-1}=0$.

(2) The symbol $(\cdot, \cdot)^{1 / n}:$ Val $\times$ Res $\rightarrow$ Val for the function sending $(x, \xi)$ to the (unique) element $y$ with $y^{n}=x$ and $\overline{\mathrm{ac}}(y)=\xi$ whenever $\xi^{n}=\overline{\mathrm{ac}}(x)$ and $\operatorname{ord}(x) \equiv 0 \bmod n$, and to 0 otherwise.

(3) The symbol $h_{n}: \mathrm{Val}^{n+1} \times$ Res $\rightarrow$ Val for the function sending $\left(a_{0}, \ldots, a_{n}, \xi\right)$ to the (unique) element $y$ satisfying ord $(y)=0, \overline{\mathrm{ac}}(y)=\xi$ and $\sum_{i=0}^{n} a_{i} y^{i}=0$ whenever $\xi \neq 0$, ord $\left(a_{i}\right) \geq 0, \sum_{i \in I} \overline{\mathrm{ac}}\left(a_{i}\right) \xi^{i}=0$, and $\sum_{i \in I} i \overline{\mathrm{ac}}\left(a_{i}\right) \xi^{i-1} \neq 0$ for $I=\left\{i \mid \operatorname{ord}\left(a_{i}\right)=0\right\}$, and to 0 otherwise.

The following is a fundamental structure result for definable functions with values in the valued field. Its proof uses an analogue of Lemma 3.7 of [30] which will be contained in [5].

7.5.1. Theorem. - Let $f: X \rightarrow h[1,0,0]$ be a morphism in Def $_{k}$. Then there exists a definable isomorphism $g: X \rightarrow X^{\prime} \subset X[0, n, r]$ over $X$ and a $\mathcal{L}_{\mathrm{DP}}^{*}$-term $\tilde{f}$ in variables running over $X^{\prime}$ such that $f=\tilde{f} \circ g$.

Proof. - Let $\varphi$ be a formula describing the graph of $f$ and suppose that $\varphi$ is of the form (3.3.2) as in the proof of Theorem 3.3.1. Let $p_{j}$ be all polynomials in the Val-sort which appear in $\varphi$. With exactly the same proof as the proof of the Denef-Pas cell decomposition in [30] where one replaces the words strongly definable function by $\mathcal{L}_{\mathrm{DP}}^{*}$-term (also in Lemma 3.7 of [30], cf. [5]) and assuming quantifier elimination, one shows that there exists a cell decomposition of $X[1,0,0]$ adapted to $\operatorname{ord}\left(p_{j}\right)$ for each $j$ such that the centers $c_{i}$ of the occurring cells $Z_{i}$ are $\mathcal{L}_{\mathrm{DP}^{-}}^{*}$ terms. For each cell $Z_{i}$, let $Z_{i}^{\prime}$ be $\lambda_{i}^{-1}\left(\operatorname{Graph}\left(c_{i}\right)\right) \cap \operatorname{Graph}(f)$, where $\lambda_{i}$ is the representation of $Z_{i}$. Clearly each $Z_{i}^{\prime}$ is a 0 -cell with presentation the restriction of $\lambda_{i}$ to $Z_{i}^{\prime}$. It follows from the description (3.3.2) of $\varphi$ that for each point $x$ in $X$ the point $f(x)$ is a zero of at least one of the polynomials $p_{i}(x, \cdot)$, and by cell decomposition that at least one of the centers gives this zero. Hence, the cells $Z_{i}^{\prime}$ form a partition of the graph of $f$ and one concludes that the restriction of $f$ to each of finitely many pieces in a partition of $X$ satisfies the statement. One can glue $s$ pieces together using extra parameters contained in the definable subassignment $A:=\left\{\xi \in h[0,1,0]^{s} \mid \sum_{i} \xi_{i}=1 \wedge\left(\xi_{i}=0 \vee \xi_{i}=1\right)\right\}$ to index the pieces, by noting that for each element $a$ in $A$ there exists a definable morphism $A \rightarrow h[1,0,0]$, given by a $\mathcal{L}_{\mathrm{DP}}^{*}$-term, which is the characteristic function of $\{a\}$.

Let $K$ be a field. A subset $B$ of $K((t))$ of the form $c+t^{\alpha} K \llbracket t \rrbracket$ is called a ball of volume $\mathbb{L}^{-\alpha}$. A function $f: B \rightarrow K((t))$ is called strictly analytic if there exists a power series $\varphi:=\sum_{i \in \mathbb{N}} a_{i} x^{i}$ in $K((t)) \llbracket x \rrbracket$ converging on $t^{\alpha} K \llbracket t \rrbracket$, equivalently, $\lim _{i \mapsto \infty}\left(\operatorname{ord} a_{i}+i \alpha\right)=\infty$, such that $f(c+y)=\varphi(y)$ for every $y$ in $t^{\alpha} K \llbracket t \rrbracket$. Note that 
this definition is independent of the choice of the center $c$ and that if $f$ is strictly analytic on $B$, its restriction to any ball contained in $B$ is also strictly analytic.

7.5.2. Lemma. - Let $K$ be a field of characteristic zero. Let $f$ be strictly analytic on a ball $B \subset K((t))$ of volume $\mathbb{L}^{-\beta}$ for some $\beta$ in $\mathbb{Z}$, and write $f^{\prime}$ for its derivative. Suppose that there exists $\alpha$ in $\mathbb{Z}$ such that ord $f^{\prime}(x)=\alpha$ for every $x$ in $B$. Then the image of $f$ is contained in a ball of volume $\mathbb{L}^{-\alpha-\beta}$ and cannot be contained in a ball of volume $\mathbb{L}^{-\alpha-\beta-1}$. For every $x_{0}$ in $t B$, the restriction of $f$ to $x_{0}+t B$ is a bianalytic bijection onto a ball of volume $\mathbb{L}^{-\alpha-\beta-1}$ with strictly analytic inverse. Also, $\operatorname{ord}(f(z)-f(y))=\alpha+\operatorname{ord}(z-y)$ for all $y, z$ in $x_{0}+t B$ and $x_{0}$ in $B$.

Proof. - We may assume that $B=t^{\beta} K \llbracket t \rrbracket$. Write $f=\sum_{i} a_{i} x^{i}$ in $K((t)) \llbracket x \rrbracket$. By replacing $x$ by $x / t^{\beta}$ and $f(x)$ by $\left(f(x)-a_{0}\right) / t^{\alpha}$ we may suppose that $a_{0}=\alpha=\beta=0$. First we prove that ord $a_{1}=0$ and ord $a_{i} \geq 0$ for all $i$. Let $I$ be $\left\{i \mid \operatorname{ord} a_{i}=\right.$ $\left.\min \left(\text { ord } a_{j}\right)_{j}\right\}$, let $p$ be the polynomial $\sum_{i \in I} a_{i} x^{i}$ and let $p^{\prime}$ be its derivative. If $p^{\prime}=a_{1}$ this is trivial. Suppose that the degree of $p^{\prime}$ is $>0$. Since $K$ is infinite there exists for any $b$ in $K$ an element $y_{0}$ in $K$ such that $\sum_{i \in I, i>0} i \overline{\mathrm{ac}}\left(a_{i}\right) y_{0}^{i-1} \neq b$. Taking $b=0$, it follows that ord $a_{i}=0$ for $i$ in $I$. Thus, 1 belongs to $I$ since otherwise ord $f^{\prime}(0)>0$. Fix $c$ in $K((t))$. Taking $b=\overline{\mathrm{ac}}(c)$ if ord $c=0$ and $b=0$ otherwise, it follows that the image of $f$ cannot be contained in the set $c+t K \llbracket t \rrbracket$.

Now fix $x_{0}$ in $K$. It is clear that $f$ maps $x_{0}+t K \llbracket t \rrbracket$ into $f\left(x_{0}\right)+t K \llbracket t \rrbracket$. The statement about bianaliticity is well known and follows from the Inverse Function Theorem for complete fields, stated in [26], Corollary 2.2.1(ii). The statement about the orders follows easily by developing $f$ into power series around $z$.

7.5.3. Theorem. - Let $X$ be in $\operatorname{Def}_{k}, Z$ be a definable subassignment of $X[1,0,0]$, and let $f: Z \rightarrow h[1,0,0]$ be a definable morphism. Then there exists a cell decomposition of $Z$ into cells $Z_{i}$ such that the following conditions hold for every $\xi$ in $C_{i}$, for every $K$ in $\operatorname{Field}_{k(\xi)}$, and for every 1-cell $Z_{i}$ with presentation $\lambda_{i}: Z_{i} \rightarrow Z_{C_{i}}=Z_{C_{i}, \alpha_{i}, \xi_{i}, c_{i}}$ and with projections $p_{i}: Z_{C_{i}} \rightarrow C_{i}, \pi_{i}: Z_{C_{i}} \rightarrow h[1,0,0]:$

(1) The set $\pi_{i}\left(p_{i}^{-1}(\xi)\right)(K)$ is either empty or a ball of volume $\mathbb{L}^{-\alpha_{i}(\xi)-1}$.

(2) When $\pi_{i}\left(p_{i}^{-1}(\xi)\right)(K)$ is nonempty, the function

$$
g_{\xi, K}: \pi_{i}\left(p_{i}^{-1}(\xi)\right)(K) \rightarrow K((t)): x \longmapsto f \circ \lambda^{-1}(\xi, x)
$$

is strictly analytic.

We can furthermore ensure that either $g_{\xi, K}$ is constant or (3), (4), and (5) hold.

(3) There exists a definable morphism $\beta_{i}: C_{i} \rightarrow h[0,0,1]$ such that

$$
\text { ord } \frac{\partial}{\partial x} g_{\xi, K}(x)=\beta_{i}(\xi)
$$

for all $x$ in $\pi_{i}\left(p_{i}^{-1}(\xi)\right)(K)$.

(4) When $\pi_{i}\left(p_{i}^{-1}(\xi)\right)(K)$ is nonempty, the map $g_{\xi, K}$ is a bijection onto a ball of volume $\mathbb{L}^{-\alpha_{i}(\xi)-1-\beta_{i}(\xi)}$.

(5) For every $x, y$ in $\pi_{i}\left(p_{i}^{-1}(\xi)\right)(K)$, ord $\left(g_{\xi, K}(x)-g_{\xi, K}(y)\right)=\beta_{i}(\xi)+\operatorname{ord}(x-y)$. 
Note that, in this theorem, $\alpha_{i}(\xi)$ and $\beta_{i}(\xi)$ are independent of $K$.

Proof of Theorem 7.5.3. - Statement (1) holds automatically if $\alpha_{i}$ is a coordinate function in the image of the presentation $\lambda_{i}$.

Take a 1-cell $Z$ with presentation $\lambda: Z \rightarrow Z_{C}$ and projection $p: Z_{C} \rightarrow C$. By Theorem 7.5.1 we may suppose that $f \circ \lambda^{-1}$ is given by a $\mathcal{L}_{\mathrm{DP}}^{*}$-term. We shall now prove statement (2) by induction on the complexity of the term $f \circ \lambda^{-1}$. Fix $\xi_{0}$ in $C$. The case where $\pi_{i}\left(p_{i}^{-1}\left(\xi_{0}\right)\right)(K)$ is empty being clear, we may assume after translation and homothety that $\pi_{i}\left(p_{i}^{-1}\left(\xi_{0}\right)\right)(K)$ is in fact the ball $K \llbracket t \rrbracket$. Consider the term $a^{-1}$ with $a$ a term for which the statement already holds. By cell decomposition we may assume that ord $(a)$ and $\overline{\operatorname{ac}}(a)$ only depend on $\xi_{0}$. We may also assume $a$ is non zero. Let us denote by $\tilde{a}$ the function induced by $a$ on the ball $K \llbracket t \rrbracket$. We may write

$$
\tilde{a}=t^{\alpha} \eta\left(1+\sum_{j \geq 1} P_{j}(x) t^{j}\right)
$$

with $\alpha$ in $\mathbb{Z}, \eta$ non zero in $K$, and $P_{j}$ polynomials in $K[x]$. We use here the fact that $K$ is infinite. Note also that a series in $K \llbracket t \rrbracket \llbracket x \rrbracket$ converges on the ball $K \llbracket t \rrbracket$ if and only if it lies in $K[x] \llbracket t \rrbracket$. Since $1+\sum_{j \geq 1} P_{j}(x) t^{j}$ is a unit in the ring $K[x] \llbracket t \rrbracket$, the result follows in this case. Similarly, consider a term $(a, \xi)^{1 / n}$, with $a$ a term for which the statement already holds. As before we may assume that the function $\tilde{a}$ induced by $a$ on the ball $\pi_{i}\left(p_{i}^{-1}\left(\xi_{0}\right)\right)(K)$ is of the form (17.5.1). Furthermore we may assume that $\alpha$ lies in $n \mathbb{Z}$ and $\eta=\xi^{n}$. The result follows since the series $1+\sum_{j \geq 1} P_{j}(x) t^{j}$ has a unique $n$-th root of the form $1+\sum_{j \geq 1} Q_{j}(x) t^{j}$ in the ring $K[x] \llbracket t \rrbracket$. Now assume the term is $h_{n}\left(a_{0}, \ldots, a_{n}, \xi\right)$ where the $a_{i}$ are terms for which the statement holds and $\xi_{0} \in X$. We may assume by cell decomposition that $\xi$, ord $\left(a_{i}\right)$ and $\overline{\operatorname{ac}}\left(a_{i}\right)$ only depend on $\xi_{0}$. Denoting by $\tilde{a}_{i}$ the function induced by $a_{i}$ on the ball $\pi_{i}\left(p_{i}^{-1}\left(\xi_{0}\right)\right)(K)$, we may write

$$
\tilde{a}_{i}=t^{\alpha_{i}} \eta_{i}\left(1+\sum_{j \geq 1} P_{i, j}(x) t^{j}\right)
$$

with $P_{i, j}$ in $K[x]$. We may assume that $\alpha_{i} \geq 0$ for all $i, \sum_{i \in I} \eta_{i} \xi^{i}=0$, and $\sum_{i \in I} i \eta_{i} \xi^{i-1} \neq 0$, where $I$ denotes the set of $i$ 's with $\alpha_{i}=0$. By the usual proof of Hensel's Lemma by successive approximations modulo higher powers of $t$, one gets that there exists universal polynomials $Q_{j}$ in $K\left[x_{i, \ell}\right]_{\substack{0 \leq i \leq n \\ 1 \leq \ell \leq j}}$ such that $h_{n}\left(\tilde{a}_{0}, \ldots, \tilde{a}_{n}, \xi\right)$ is equal to

$$
h_{n}\left(\tilde{a}_{0}, \ldots, \tilde{a}_{n}, \xi\right)=\xi+\sum_{j \geq 1} Q_{j}\left(P_{i, \ell}(x)\right) t^{j}
$$

from which the assertion follows. This concludes the proof of (2), the result being clear for the remaining types of terms of the forms,,$+- \cdot$, and constants.

Statement (3) follows easily: using Theorem 3.2.1 (iii) and general model theory, there is a definable morphism $g$ which is, almost everywhere, equal to the derivative of $f$ with respect to the $h[1,0,0]$-variable; then take a refinement adapted to ord $g$. 
Clearly (4) is a definable condition on $\xi$ in $C_{i}$. Let $C_{i 1}$ be the definable subassignment of $C_{i}$ given by condition (4) and $C_{i 2}$ its complement in $C_{i}$. Set $X_{j}:=p_{i}^{-1}\left(C_{i j}\right)$ for $j=1,2$; these are cells by Lemma 7.2.5. It is enough to prove statement (4) for the $X_{j}$ and the restrictions $f_{\mid X_{j}}$. For $X_{1}$ and $f_{\mid X_{1}}$, statement (4) is clear and (5) is automatically true by (2), (4), and Lemma 7.5.2. By Lemma 7.5.2 and the construction of $X_{2}$, for $\xi$ in $C_{i 2}, p_{i}^{-1}(\xi)(K)$, if nonempty, is mapped under $f$ into a proper subset of a ball of volume $\mathbb{L}^{-\alpha_{i}(\xi)-1-\beta_{i}(\xi)}$ for every $K$ in Field $k_{k(\xi)}$. Let $Y$ be the definable subassignment of $C_{i 2}[1,0,0]$ determined by $\left(\exists x \in X_{2}\right) y=\left(p_{i} \circ \lambda_{i}(x), f(x)\right)$. If we now apply cell decomposition to $Y$, then the fibers of these cells will be strictly smaller balls than those of volume $\mathbb{L}^{-\alpha_{i}(\xi)-1-\beta_{i}(\xi)}$, by the definition of cells and the construction. By a fiber product argument, we may assume that the new parameters we just obtained, as well as those for $C_{i}$, are already present as coordinate functions for $X_{2}$. If we now apply again cell decomposition to $X_{2}$, the fibers of the cells in $X_{2}$ are strictly smaller balls than those of volume $\mathbb{L}^{-\alpha_{i}(\xi)-1}$ by Lemma 7.5.2. An application of Lemma 7.5 .2 shows that (4) and (5) for $X_{2}$ and $f_{\mid X_{2}}$ hold on this cell decomposition of $X_{2}$.

7.5.4. Corollary. - Let $f: S[1,0,0] \rightarrow S[1,0,0]$ be a definable isomorphism over $S$ and let $\pi: S[1,0,0] \rightarrow h[1,0,0]$ be the projection. Then there exists a finite partition of $S[1,0,0]$ into cells $Z_{i}$ with presentation $\lambda_{i}: Z_{i} \rightarrow Z_{C_{i}}$ and projection $p_{i}: Z_{C_{i}} \rightarrow C_{i}$ such that the $f\left(Z_{i}\right)$ are cells with presentation $\left(p_{i} \circ \lambda_{i} \circ f^{-1}, \pi\right): x \mapsto$ $\left(p_{i} \circ \lambda_{i} \circ f^{-1}(x), \pi(x)\right)$ and such that (1) up to (5) of Theorem 7.5.3 are fulfilled for each 1-cell $Z_{i}$ and the map $\pi \circ f$.

Moreover, one can take the $Z_{i}$ and $f\left(Z_{i}\right)$ adapted to $f^{*}(\varphi)$ and $\varphi$ for given $\varphi$ in $\mathscr{C}_{+}(S[1,0,0])$.

Proof. - First apply Theorem 7.5 .3 to $S[1,0,0]$ and the function $\pi \circ f$. Then apply cell decomposition to $f^{*}(\varphi)$ to refine the obtained cells. The corollary follows.

7.5.5. Remark. - Alternatively to the proofs of section 3 , based on the work by van den Dries, one could proof many of the results of section 3 using cell decomposition and Theorems 7.5.1 and 7.5.3.

\section{Volume forms and Jacobians}

8.1. Differential forms on definable subassignments. - Let $W$ be of the form $W=\mathcal{X} \times X \times \mathbb{Z}^{r}$ with $\mathcal{X}$ a $k((t))$-variety and $X$ a $k$-variety. Let $h$ be a definable subassignment of $h_{W}$. Denote by $\mathcal{A}(h)$ the ring of definable functions $h \rightarrow h_{\mathbb{A}_{k(t))}^{1}}$ on $h$. We want to define, for every integer $i$ in $\mathbb{N}$, an $\mathcal{A}(h)$-module $\Omega^{i}(h)$ of definable $i$-forms on $h$, which we do in (8.1.1).

First consider a $k((t))$-variety $\mathcal{Y}$ and the sheaf $\Omega_{\mathcal{Y}}^{i}$ of degree $i$ algebraic differential forms on $\mathcal{Y}$, namely, the $i$-th exterior product of the sheaf $\Omega_{\mathcal{Y}}^{1}$ of Kähler differentials. Denote by $\mathcal{A}_{\mathcal{Y}}$ the Zariski sheaf associated to the preshea $U \mapsto \mathcal{A}\left(h_{U}\right)$ on $\mathcal{Y}$. Both

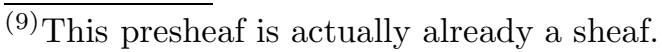


$\Omega_{\mathcal{Y}}^{i}$ and $\mathcal{A}_{\mathcal{Y}}$ are sheaves of $\mathcal{O}_{\mathcal{Y}}\left(=\Omega_{\mathcal{Y}}^{0}\right)$-modules, so we can consider the sheaf

$$
\Omega_{h_{\mathcal{Y}}}^{i}:=\mathcal{A}_{\mathcal{Y}} \otimes_{\mathcal{O}_{\mathcal{Y}}} \Omega_{\mathcal{Y}}^{i}
$$

of definable degree $i$ differential forms on $\mathcal{Y}$. Note that, in general, the module of global sections $\Omega_{h_{\mathcal{Y}}}^{i}(\mathcal{Y})$ is much bigger than $\mathcal{A}_{\mathcal{Y}}(\mathcal{Y}) \otimes_{\mathcal{O}_{\mathcal{Y}}(\mathcal{Y})} \Omega_{\mathcal{Y}}^{i}(\mathcal{Y})$.

Now let $\mathcal{Y}$ be the subvariety of $\mathcal{X}$ which is the Zariski closure of the image of $h$ under the projection $\pi: h_{W} \rightarrow h_{\mathcal{X}}$. Using the ring morphism $\mathcal{A}\left(h_{\mathcal{Y}}\right) \rightarrow \mathcal{A}(h): f \mapsto$ $f \circ \pi$, we define the $\mathcal{A}(h)$-module $\Omega^{i}(h)$ of definable $i$-forms on $h$ as

$$
\Omega^{i}(h):=\mathcal{A}(h) \otimes_{\mathcal{A}\left(h_{\mathcal{Y}}\right)} \Omega_{h \mathcal{Y}}^{i}(\mathcal{Y}) .
$$

Note that $\Omega^{0}(h)=\mathcal{A}(h)$.

Let $d$ be the $K$-dimension of $h$. We denote by $\mathcal{A}^{<}(h)$ the ideal of $\mathcal{A}(h)$ consisting of definable functions on $h$ vanishing on the complement of a definable subassignment of $K$-dimension $<d$, and we set

$$
\tilde{\Omega}^{d}(h):=\Omega^{d}(h) /\left(\mathcal{A}^{<}(h) \Omega^{d}(h)\right) .
$$

It is a free $\mathcal{A}(h)$-module of rank 1 since, for $\mathcal{Y}$ a $k((t))$-variety of dimension $d$, the sheaf $\Omega_{\mathcal{Y}}^{d}$ is locally free of rank one away from the singular locus.

Let $f: h^{\prime} \rightarrow h$ be a definable morphism between two definable subassignments. Assume $h$ and $h^{\prime}$ are both of $K$-dimension $d$ and that the fibers of $f$ all have $K$ dimension 0 . Under these conditions, we define a natural pullback morphism

$$
f^{*}: \tilde{\Omega}(h) \longrightarrow \tilde{\Omega}\left(h^{\prime}\right)
$$

as follows. Let $\omega$ be in $\tilde{\Omega}(h)$. By Theorem 3.2.1 (iii), there exist definable subassignments $Z \subset h, Z^{\prime} \subset h^{\prime}$ such that, for each $K$ in $\operatorname{Field}_{k}, Z(K)$ and $Z^{\prime}(K)$ are $K((t))$-analytic manifolds, $\operatorname{Kdim}(h \backslash Z)<\operatorname{Kdim} h, \operatorname{Kdim}\left(h^{\prime} \backslash Z^{\prime}\right)<\operatorname{Kdim} h^{\prime}$, $f_{K}:=f(K)_{\mid Z^{\prime}(K)}$ is $K((t))$-analytic on $Z^{\prime}(K)$, and such that $\omega$ induces a $K((t))$ analytic $d$-form $\omega_{K}$ on $Z(K)$. Using partial differentials with respect to local coordinates on $Z(K)$, it is clear that there exists a unique definable $d$-form $\omega^{\prime}$ in $\tilde{\Omega}\left(h^{\prime}\right)$ which induces a $K((t))$-analytic $d$-form $\omega_{K}^{\prime}$ on $Z^{\prime}(K)$ with $\omega_{K}^{\prime}=f_{K}^{*}\left(\omega_{K}\right)$ for each $K$. Define $f^{*}(\omega)$ as the class of $\omega^{\prime}$ in $\tilde{\Omega}\left(h^{\prime}\right)$.

8.2. Volume forms on definable subassignments. - Let $h$ be a definable subassignment of $h_{W}, W=\mathcal{X} \times X \times \mathbb{Z}^{r}$. Assume $h$ is of $K$-dimension $d$. There is a canonical morphism of commutative semigroups

$$
\lambda: \mathcal{A}(h) \longrightarrow C_{+}^{d}(h)
$$

sending a function $f$ to the class of $\mathbb{L}^{- \text {ord } f}$, with the convention $\mathbb{L}^{-\infty}=0$. Define the space $|\tilde{\Omega}|_{+}(h)$ of definable positive volume forms on $h$ as the quotient of the

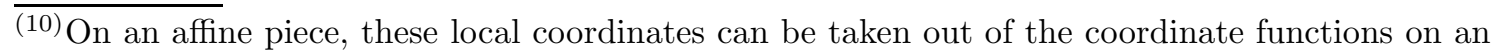
embedding affine space. 
free abelian semi-group on symbols $(\omega, g)$, with $\omega$ in $\tilde{\Omega}^{d}(h)$ and $g$ in $C_{+}^{d}(h)$, by the relations:

$$
\begin{aligned}
(f \omega, g) & =(\omega, \lambda(f) g) \\
\left(\omega, g+g^{\prime}\right) & =(\omega, g)+\left(\omega, g^{\prime}\right) \\
(\omega, 0) & =0
\end{aligned}
$$

for all $f$ in $\mathcal{A}(h)$ and $g^{\prime}$ in $C_{+}^{d}(h)$. We shall write $g|\omega|$ for the class of $(\omega, g)$, so that $g|f \omega|=g \mathbb{L}^{- \text {ord } f}|\omega|$. In particular, if $\omega$ is a differential form in $\tilde{\Omega}^{d}(h)$ (or in $\left.\Omega^{d}(h)\right)$, we shall denote by $|\omega|$ the class of $(\omega, 1)$ in $|\tilde{\Omega}|_{+}(h)$. If $h^{\prime}$ is a definable subassignment of $h$, there is a natural restriction morphism $|\tilde{\Omega}|_{+}(h) \rightarrow|\tilde{\Omega}|_{+}\left(h^{\prime}\right)$. When $h^{\prime}$ is of $K$-dimension $d$, it is induced by restriction of differential forms and Functions. When $h^{\prime}$ is of $K$-dimension $<d$, we define it to be the zero morphism.

Note that $|\tilde{\Omega}|_{+}(h)$ has a natural structure of $C_{+}^{d}(h)$-module. We shall say an element $|\omega|$ with $\omega$ in $\tilde{\Omega}^{d}(h)$ is a gauge form, if it is a generator of this $C_{+}^{d}(h)$ module. Gauge forms always exist, since $\tilde{\Omega}^{d}(h)$ is a free $\mathcal{A}(h)$-module of rank 1 .

Replacing $C_{+}^{d}(h)$ by $C^{d}(h)$, one defines similarly the $C^{d}(h)$-module $|\tilde{\Omega}|(h)$.

Let $f: h^{\prime} \rightarrow h$ be a definable morphism between two definable subassignments. Assume $h$ and $h^{\prime}$ are both of $K$-dimension $d$ and that the fibers of $f$ all have $K$ dimension 0. Under these assumptions, pullback of functions induces a morphism $f^{*}: C_{+}^{d}(h) \rightarrow C_{+}^{d}\left(h^{\prime}\right)$. Still under these conditions, the pullbacks $f^{*}: \tilde{\Omega}(h) \longrightarrow \tilde{\Omega}\left(h^{\prime}\right)$ and $f^{*}: C_{+}^{d}(h) \rightarrow C_{+}^{d}\left(h^{\prime}\right)$ induce a natural pullback morphism

$$
f^{*}:|\tilde{\Omega}|_{+}(h) \longrightarrow|\tilde{\Omega}|_{+}\left(h^{\prime}\right),
$$

defined by sending the class of $(\omega, g)$ to the class of $\left(f^{*}(\omega), f^{*}(g)\right)$.

8.3. Canonical volume forms. - Let $h$ be a definable subassignment of $h[m, n, r]$ of $K$-dimension $d$. We denote by $x_{1}, \ldots, x_{m}$ the coordinates on $\mathbb{A}_{k(t))}^{m}$ and we consider the $d$-forms $\omega_{I}:=d x_{i_{1}} \wedge \cdots \wedge d x_{i_{d}}$ for $I=\left\{i_{1}, \ldots, i_{d}\right\} \subset\{1, \ldots m\}$, $i_{1}<\cdots<i_{d}$. We denote by $\left|\omega_{I}\right|_{h}$ the image of $\omega_{I}$ in $|\tilde{\Omega}|_{+}(h)$.

8.3.1. Lemma-Definition. - There is a unique element $\left|\omega_{0}\right|_{h}$ in $|\tilde{\Omega}|_{+}(h)$, the canonical volume form, such that, for every $I$, there exists $\mathbb{Z}$-valued definable functions $\alpha_{I}$ and $\beta_{I}$ on $h$, with $\beta_{I}$ only taking as values 1 and 0 , such that $\alpha_{I}+\beta_{I}>0$ on $h,\left|\omega_{I}\right|_{h}=\beta_{I} \mathbb{L}^{-\alpha_{I}}\left|\omega_{0}\right|_{h}$ in $|\tilde{\Omega}|_{+}(h)$, and such that $\inf _{I} \alpha_{I}=0$.

Proof. - Uniqueness is clear. Fix a gauge form $|\omega|$ on $h$. We may write $\left|\omega_{I}\right|_{h}=$ $\beta_{I} \mathbb{L}^{-\gamma_{I}}|\omega|$, with $\gamma_{I}$ and $\beta_{I} \mathbb{Z}$-valued definable functions on $h$, and $\beta_{I}$ only taking 0 and 1 as values. Clearly we may suppose that $\beta_{I}+\gamma_{I}>\inf _{I} \gamma_{I}$. If one sets $\alpha=\inf _{I} \gamma_{I}$, then $\left|\omega_{0}\right|_{h}:=\mathbb{L}^{-\alpha}|\omega|$ satisfies the required property.

We call $\left|\omega_{0}\right|_{h}$ the canonical volume form on $h$. It is a gauge form on $h$. It is an analogue of the canonical volume form defined by Serre in [35] in the $p$-adic case. 
8.4. Order of jacobian. - Let $f: X \rightarrow Y$ be a definable morphism between two definable subassignments of $h[m, n, r]$ and $h\left[m^{\prime}, n^{\prime}, r^{\prime}\right]$, respectively. Assume $X$ and $Y$ are both of $K$-dimension $d$ and that the fibers of $f$ all have $K$-dimension 0 . By (8.2.1) we may consider $f^{*}\left|\omega_{0}\right|_{Y}$ and we may write

$$
f^{*}\left|\omega_{0}\right|_{Y}=\mathbb{L}^{-\operatorname{ordjac} f}\left|\omega_{0}\right|_{X}
$$

with ordjac $f$ a $\mathbb{Z}$-valued function on $X$ defined outside a definable subassignment of $K$-dimension $<d$. Since, basically, ordjac comes from calculating (the order of) partial derivatives in the valued field, the restriction of ordjac $f$ to a definable subassignment of $K$-dimension $<d$ is a definable morphism. Thus, ordjac $f$ and $\mathbb{L}^{\text {ordjac } f}$ make sense as Functions in $C_{+}^{d}(X)$.

8.4.1. Proposition (Chain rule for ordjac). - Let $f: X \rightarrow Y$ and $g: Y \rightarrow Z$ be definable functions between definable subassignments of $K$-dimension d. Assume the fibers of $f$ and $g$ all have $K$-dimension 0 . Then

$$
\operatorname{ordjac}(g \circ f)=(\operatorname{ordjac} f)+((\operatorname{ordjac} g) \circ f)
$$

outside a definable subassignment of $K$-dimension $<d$.

Proof. - Follows directly from the chain rule for the pullback of usual differential forms.

8.5. Relative variants. - Let $h \rightarrow \Lambda$ and $h^{\prime} \rightarrow \Lambda$ be morphisms in $\operatorname{Def}_{k}$. Assume that $h \rightarrow \Lambda$ and $h^{\prime} \rightarrow \Lambda$ are equidimensional of relative $K$-dimension $d$. Let $f: h^{\prime} \rightarrow h$ be a definable morphism whose fibers have dimension 0 and which commutes with the projections to $\Lambda$. On each fiber $h_{\lambda}$ and $h_{\lambda}^{\prime}$ for $\lambda$ in $\Lambda$, let $\left|\omega_{0}\right| h_{\lambda}$, resp. $\left|\omega_{0}\right|_{h_{\lambda}^{\prime}}$, be the canonical volume form in $|\tilde{\Omega}|_{+}\left(h_{\lambda}\right)$ and $|\tilde{\Omega}|_{+}\left(h_{\lambda}^{\prime}\right)$. For every $\lambda$ in $\Lambda$, let $f_{\lambda}: h_{\lambda}^{\prime} \rightarrow h_{\lambda}$ be the map induced by $f$ and write ordjac $f_{\lambda}$ for the Function in $C_{+}^{d}\left(h_{\lambda}^{\prime}\right)$ defined in 8.4. As in the non-relative setting 8.4, behind this are partial derivatives with respect to valued field variables, which are compatible with definability by the $\varepsilon, \delta$ definitions of partial derivatives. Thus, by construction there exists a unique Function $\operatorname{ordjac}_{\Lambda} f$ in $C_{+}^{d}\left(h^{\prime} \rightarrow \Lambda\right)$ which is the class of a definable morphism $h^{\prime} \rightarrow \mathbb{Z}$ such that the fiber of $\operatorname{ordjac}_{\Lambda} f$ at $\lambda$ equals ordjac $f_{\lambda}$ for every $\lambda$ in $\Lambda$.

We have a commutative diagram:

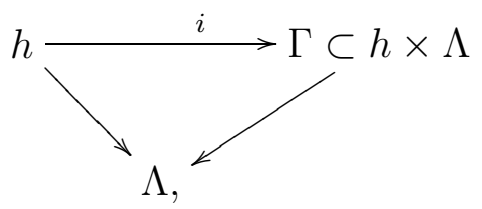

where $i$ denotes the isomorphism to the graph $\Gamma$ of $h \rightarrow \Lambda$. By construction, $\operatorname{ordjac}_{\Lambda} i=0$ holds.

8.5.1. Remark. - It is also possible to define relative analogues of $\Omega^{i}(h), \tilde{\Omega}^{d}(h)$, $|\tilde{\Omega}|_{+}(h),|\tilde{\Omega}|(h)$, pullbacks, and $\left|\omega_{0}\right|_{h}$. We won't pursue this. 
8.6. Models and volume forms. - The following construction will not be needed until $\S\left[16\right.$. Let $\mathcal{X}^{0}$ be an algebraic variety over Spec $k \llbracket t \rrbracket$, say flat over Spec $k \llbracket t \rrbracket$. Set $\mathcal{X}:=\mathcal{X}^{0} \otimes_{\text {Spec } k \llbracket t \rrbracket} \operatorname{Spec} k((t))$. In other words $\mathcal{X}$ is the generic fiber of $\mathcal{X}^{0}$ and $\mathcal{X}^{0}$ is a model of $\mathcal{X}$. Assume $\mathcal{X}$ is of dimension $d$. Let us denote by $U^{0}$ the largest open subset of $\mathcal{X}^{0}$ on which the sheaf $\Omega_{\mathcal{X}^{0} \mid k \llbracket t \rrbracket}^{d}$ is locally free of rank 1 over $k \llbracket t \rrbracket$. Its generic fiber $U:=U^{0} \otimes_{\text {Spec } k \llbracket t \rrbracket}$ Spec $k((t))$ may be identified with the smooth locus of $\mathcal{X}$ when $\mathcal{X}$ is of pure dimension $d$. Let us choose a finite cover of $U^{0}$ by open subsets $U_{i}^{0}$ on which the sheaf $\Omega_{\mathcal{X}^{0} \mid k \llbracket t \rrbracket}^{d}$ is generated by a non zero form $\omega_{i}$ in $\Omega_{U_{i}^{0} \mid k \llbracket t \rrbracket}^{d}\left(U_{i}^{0}\right)$. Each form $\omega_{i}$ gives rise to a volume form $\left|\omega_{i}\right|$ in $|\Omega|_{+}\left(h_{U_{i}}\right)$, where $U_{i}$ denotes the generic fiber of $U_{i}$. The subsets $U_{i}$ form an open cover of $U$. Clearly there exists a unique element $\left|\omega_{0}\right|$ in $|\tilde{\Omega}|_{+}\left(h_{\mathcal{X}}\right)$ such that $\left|\omega_{0}\right|_{\mid h_{U_{i}}}=\left|\omega_{i}\right|$ in $|\tilde{\Omega}|_{+}\left(h_{U_{i}}\right)$. Furthermore, $\left|\omega_{0}\right|$ only depends on the model $\mathcal{X}^{0}$, not on the choice of the cover by open subsets $U_{i}^{0}$.

\section{Integrals in dimension one}

This section is only needed to show that integrals in dimension 1 , as axiomatized by Theorems 10.1.1 and14.1.1, are well defined, and satisfy a basic change of variable formula. These results will be used in the proofs of Theorems 10.1.1 and 14.1.1 and the general change of variables formulas.

9.1. Relative integrals relative to the projection $S[1,0,0] \rightarrow S$ - Let $S$ be in $\operatorname{Def}_{k}$ and let $\varphi$ be in $C_{+}(S[1,0,0] \rightarrow S)$. Since $C_{+}(S[1,0,0] \rightarrow S)=$ $\oplus_{i=0}^{1} C_{+}^{i}(S[1,0,0] \rightarrow S)$, we can write $\varphi=\left[\varphi_{0}\right]+\left[\varphi_{1}\right]$ with $\varphi_{i}$ in $\mathscr{C}_{+}(S[1,0,0])$ of relative dimension $i$ relative to the projection $S[1,0,0] \rightarrow S$ for $i=0,1$. Let $\mathcal{P}_{i}$ for $i=0,1$ be a cell decomposition of $S[1,0,0]$ adapted to $\varphi_{i}$ as in Theorem 7.2 .1 and set $\mathcal{P}_{i i}=\left\{Z \in \mathcal{P}_{i} \mid Z\right.$ is a $i$-cell $\}$. Fix $Z_{i}$ in $\mathcal{P}_{i i}$ for $i=0,1$. The cell $Z_{i}$ has a presentation $\lambda_{i}: Z_{i} \rightarrow Z_{C_{i}}$ with $Z_{C_{1}}=Z_{C_{1}, \alpha_{1}, \xi_{1}, c_{1}} \subset S\left[1, s_{1}, r_{1}\right]$, and $Z_{C_{0}}=Z_{C_{0}, c_{0}} \subset S\left[1, s_{0}, r_{0}\right]$, for some $r_{i}, s_{i} \geq 0, r_{0}=0$, some $C_{i} \subset S\left[0, s_{i}, r_{i}\right]$, and some definable morphisms $c_{i}, \alpha_{1}$, and $\xi_{1}$, for $i=1,2$. By Theorem 7.2.1, there is $\psi_{i}$ in $\mathscr{C}_{+}\left(C_{i}\right)$ such that

$$
\varphi_{i \mid Z_{i}}=\lambda_{i}^{*} p_{i}^{*}\left(\psi_{i}\right)
$$

where $p_{i}$ is the projection $Z_{C_{i}} \rightarrow C_{i}, i=0,1$. Note that $\psi_{i}$ is unique for fixed $\varphi_{i}$ since $\lambda_{i}$ is an isomorphism and $p_{i}$ is surjective. For $i=0,1$ we write $j_{i}$ for the inclusion

$$
j_{i}: C_{i} \rightarrow S\left[0, s_{i}, r_{i}\right]
$$

and $\pi_{i}$ for the projection

$$
S\left[0, s_{i}, r_{i}\right] \rightarrow S\left[0,0, r_{i}\right]
$$

9.1.1. Lemma-Definition. - The following definitions do not depend on the choice of $\lambda_{i}, i=0,1$, where we use the above notation. We set

$$
\mu_{S, Z_{0}}\left(\varphi_{0} \mathbf{1}_{Z_{0}}\right):=\pi_{0 !}\left(j_{0 !}\left(\psi_{0}\right)\right)
$$


in $\mathscr{C}_{+}(S)$. Also, we say $\varphi_{1} \mathbf{1}_{Z_{1}}$ is $S$-integrable along $Z_{1}$ if $\pi_{1 !} j_{1 !}\left(\mathbb{L}^{-\alpha_{1}-1} \psi_{1}\right)$ is $S$ integrable and if this is the case we set

$$
\mu_{S, Z_{1}}\left(\varphi_{1} \mathbf{1}_{Z_{1}}\right):=\mu_{S}\left(\pi_{1 !}\left(j_{1 !}\left(\mathbb{L}^{-\alpha_{1}-1} \psi_{1}\right)\right)\right)
$$

in $\mathscr{C}_{+}(S)$. Here, $j_{i !}, \pi_{i !}$, and $\mu_{S}$ are as in sections 5.5, 5.6, and 5.7, respectively.

9.1.2. Lemma-Definition. - The following definitions do not depend on the choice of $\varphi_{i}$ and $\mathcal{P}_{i}, i=0,1$, where we use the above notation. We say $\varphi$ is $S$ integrable if $\varphi_{1} \mathbf{1}_{Z}$ is $S$-integrable along $Z$ for each $Z$ in $\mathcal{P}_{11}$. If this is the case we define $\mu_{S}(\varphi)$ in $\mathscr{C}_{+}(S)$ as

$$
\mu_{S}(\varphi):=\sum_{i=0,1} \sum_{Z \in \mathcal{P}_{i i}} \mu_{S, Z}\left(\varphi_{i} \mathbf{1}_{Z}\right)
$$

Proof of 9.1.1 - We first prove independence from the choice of $\lambda_{0}$. Suppose there is another presentation $\lambda_{0}^{\prime}: Z_{0} \rightarrow Z_{C_{0}^{\prime}, c_{0}^{\prime}}$ and $\psi_{0}^{\prime}$ in $\mathscr{C}_{+}\left(C_{0}^{\prime}\right)$ with $\lambda_{0}^{\prime *} p_{0}^{\prime *}\left(\psi_{0}^{\prime}\right)=\varphi_{0 \mid Z_{0}}$ with $p_{0}^{\prime}: Z_{C_{0}^{\prime}, c_{0}^{\prime}} \rightarrow C_{0}^{\prime}$ the projection. Then, by the definition of 0-cells and by functoriality of the pullback, there is a definable isomorphism $f_{0}: C_{0} \rightarrow C_{0}^{\prime}$ over $S$ with $f_{0}^{*}\left(\psi_{0}^{\prime}\right)=\psi_{0}$. Now independence from the choice of $\lambda_{0}$ follows from Proposition 5.8 .6 .

Let us prove now independence from the choice of $\lambda_{1}$. Let $\lambda_{1}^{\prime}: Z_{1} \rightarrow Z_{C_{1}^{\prime}}=$ $Z_{C_{1}^{\prime}, \alpha_{1}^{\prime}, \xi_{1}^{\prime}, c_{1}^{\prime}}^{\prime} \subset S\left[1, s_{1}^{\prime}, r_{1}^{\prime}\right]$ be another presentation, for some $s_{1}^{\prime}, r_{1}^{\prime} \geq 0$ and some definable morphisms $\alpha_{1}^{\prime}, \xi_{1}^{\prime}$, and $c_{1}^{\prime}$, and $\psi_{1}^{\prime}$ in $\mathscr{C}_{+}\left(C_{1}^{\prime}\right)$ such that

$$
\varphi_{1 \mid Z_{1}}=\lambda_{1}^{\prime *} p_{1}^{\prime *}\left(\psi_{1}^{\prime}\right)
$$

where $p_{1}^{\prime}$ is the projection $Z_{C_{1}^{\prime}} \rightarrow C_{1}^{\prime}$. Write $\pi_{1}^{\prime}$ for the projection $S\left[0, s_{1}^{\prime}, r_{1}^{\prime}\right] \rightarrow$ $S\left[0,0, r_{1}^{\prime}\right]$ and $j_{1}^{\prime}$ for the inclusion $C_{1}^{\prime} \rightarrow S\left[0, s_{1}^{\prime}, r_{1}^{\prime}\right]$. By Lemma 7.3.1 (with $Z$ and $Z^{\prime}$ of Lemma 7.3.1 both equal to $Z_{1}$ here), we may suppose that there is a (unique) definable morphism $g: C_{1} \rightarrow C_{1}^{\prime}$ over $S$ such that $g \circ p_{1} \circ \lambda_{1}=p_{1}^{\prime} \circ \lambda_{1}^{\prime}$. Indeed, it is enough to compare both $\lambda_{1}$ and $\lambda_{1}^{\prime}$ with the presentation

$$
\lambda_{1}^{\prime \prime}: Z_{1} \rightarrow Z_{C_{1} \times C_{1}^{\prime}}: z \mapsto\left(p_{1} \circ \lambda_{1}(z), \lambda_{1}^{\prime}(z)\right),
$$

and by symmetry it is enough to compare $\lambda_{1}^{\prime}$ with $\lambda_{1}^{\prime \prime}$, hence we may suppose that $\lambda_{1}^{\prime \prime}=\lambda_{1}$ and $g: C_{1} \rightarrow C_{1}^{\prime}$ exists, cf. the proof of Lemma 7.3.1. By functoriality of the pullback we have $g^{*}\left(\psi_{1}^{\prime}\right)=\psi_{1}$. The result now follows from Lemma 9.1.3 and Proposition 5.8.5.

9.1.3. Lemma. - Let $S$ be in $\operatorname{Def}_{k}$ and let $X=Z_{C_{1}, \alpha_{1}, \xi_{1}, c_{1}} \subset S[1,0,0]$ be a 1-cell with basis $C_{1} \subset S$. Let $\lambda: X \rightarrow Z_{C, \alpha, \xi, c}$ be another presentation of the 1-cell $X$, with $C \subset S[0, n, r]$ and $\pi: C \rightarrow S[0,0, r]$ be the projection. Then

$$
\mu_{S}\left(\pi_{!}\left(\mathbb{L}^{-\alpha-1}\right)\right)=\mathbb{L}^{-\alpha_{1}-1} \mathbf{1}_{C_{1}}
$$

in $\mathscr{C}_{+}(S)$. 
9.1.4. Example. - Let us consider the simple case where $X$ is the 1-cell $Z_{C_{1}, \alpha_{1}, \xi_{1}, c_{1}} \subset h[1,0,0]$ with $C_{1}=h[0,0,0], \alpha_{1}=0, c_{1}=1, \xi_{1}=-1$. So $X$ is the definable subassignment of $h[1,0,0]$ given by $\operatorname{ord}(x)>0 \vee x=0$ (a ball). Another presentation for $X$ is $\lambda: X \rightarrow Z_{C, \alpha, \xi, c}$ with $C=h[0,1,0], \alpha(\eta)=1, c(\eta)=0$ when $\eta \neq 0, c(0)=t, \xi(\eta)=\eta$ when $\eta \neq 0, \xi(0)=-1$, and $\lambda(x)=(\overline{\operatorname{ac}}(x), x)$ for $x$ with $\operatorname{ord}(x)=1$ and $\lambda(x)=(0, x)$ for $x$ with $\operatorname{ord}(x)>1$. Hence, the ball $X(K)$ is partitioned into smaller balls, and there are "residue field many" of these smaller balls, that is, the smaller balls are parameterized by $C$. In this example, the formula (9.1.1) in Lemma 9.1.3 holds, since

$$
\begin{aligned}
\mu_{h[0,0,0]}\left(\pi_{!}\left(\mathbb{L}^{-\alpha-1}\right)\right) & =\mu_{h[0,0,0]}\left([C] \cdot \mathbb{L}^{-2}\right)=\mu_{h[0,0,0]}\left(\mathbb{L} \cdot \mathbb{L}^{-2}\right) \\
& =\mu_{h[0,0,0]}\left(\mathbb{L}^{-1}\right)=\mathbb{L}^{-1}=\mathbb{L}^{-\alpha_{1}-1},
\end{aligned}
$$

in $\mathscr{C}_{+}(h[0,0,0]), \mu_{h[0,0,0]}$ being trivial on $\mathscr{C}_{+}(h[0,0,0])$.

9.1.5. Example. - Let $X$ be as in Example 9.1.4. Fix $\gamma \geq 1$. Another presentation for $X$ is $\lambda: X \rightarrow Z_{C, \alpha, \xi, c}$ with $C=h_{\mathbb{G}_{k}^{m}} \times\{i \in \mathbb{Z} \mid 1 \leq i \leq \gamma\} \cup(h[0,1,0] \times$ $\{\gamma+1\}), \alpha(\eta, i)=i, c(\eta, i)=0$ when $\eta \neq 0, c(0, \gamma+1)=t^{\gamma+1}, \xi(\eta, i)=\eta$ when $\eta \neq 0, \xi(0, \gamma+1)=-1$, and $\lambda(x)=(\overline{\operatorname{ac}}(x), \operatorname{ord}(x), x)$ for $x$ with $\operatorname{ord}(x) \leq \gamma+1$ and $\lambda(x)=(0, \gamma+1, x)$ for $x$ with $\operatorname{ord}(x)>\gamma+1$ or $x=0$. The formula (9.1.1) in Lemma 9.1.3 holds, since

$$
\begin{aligned}
\mu_{h[0,0,0]}\left(\pi_{!}\left(\mathbb{L}^{-\alpha-1}\right)\right) & =\mu_{h[0,0,0]}\left(\left[\mathbb{G}_{k}^{m}\right]\left(\mathbb{L}^{-2}+\ldots+\mathbb{L}^{-\gamma-1}\right)+\mathbb{L}^{-\gamma-2}\right) \\
& =\mu_{h[0,0,0]}\left((\mathbb{L}-1)\left(\mathbb{L}^{-2}+\ldots+\mathbb{L}^{-\gamma-1}\right)+\mathbb{L}^{-\gamma-1}\right) \\
& =\mu_{h[0,0,0]}\left(\mathbb{L}^{-1}\right)=\mathbb{L}^{-1}=\mathbb{L}^{-\alpha_{1}-1}
\end{aligned}
$$

in $\mathscr{C}_{+}(h[0,0,0])$. Note that it may happen that $t^{\gamma}$ is not uniformly definable in a family but this does not pose any problems by Remark 9.1.7. This example shows how one can reduce the case where the center takes two values to the case where the center takes only one value (corresponding to the first presentation of $X$ in Example 9.1.4).

9.1.6. Remark. - Let $X$ be as in Example 9.1.4. Note that, if $X(K)$ contains a subset $Z$ of the form

$$
\operatorname{ord}(x-c)=\alpha, \quad \overline{\mathrm{ac}}(x-c)=\xi,
$$

then, necessarily $\alpha \geq 0$ and $\operatorname{ord}(c) \geq 0$. Indeed, if $\alpha<0, Z$ is too large to be contained in $X(K)$, and if $\alpha \geq 0$ and $\operatorname{ord}(c)<0, Z$ is disjoint from $X(K)$. Furthermore, if $\operatorname{ord}(c)=0$ then $\alpha=0$, and $Z=X(K)$, since $Z$ is not contained in $X(K)$ when $\operatorname{ord}(c)=0$ and $\alpha>0$. It follows that if $\lambda: X \rightarrow Z_{C, \alpha, \xi, c}$ is a presentation of $X$ such that $\operatorname{ord}(c)=0$ on $C$, necessarily $C$ should be $h[0,0,0]$, and the presentation is similar to the presentation $\lambda_{1}$ with $c_{1}=1$ replaced by $c$ and $\xi_{1}=-1$ replaced by $-\overline{\mathrm{ac}}(c)$.

9.1.7. Remark. - A function $\varphi$ in $\mathscr{C}_{+}\left(Z \times Z^{\prime}\right)$ with $Z$ and $Z^{\prime}$ some definable subassignments is said not to depend on the $Z^{\prime}$-variables when $\varphi$ can be build up 
(in finitely many steps) using formulas which do not involve variables from $Z^{\prime}$. Clearly $\varphi$ then defines a unique function in $\mathscr{C}_{+}(Z)$ by restriction in the obvious way.

We shall prove Lemma 9.1 .3 by essentially reducing to the previous examples, using the previous remarks.

Proof of Lemma 9.1.3. - We start by assuming $S=h[0,0,0]$ for simplicity. Let $K$ be a field containing $k$ such that $C_{1}(K)$ is nonempty, i.e. a point $\eta_{1}(K)$. Note that $c(K): C(K) \rightarrow K((t))$ has finite image: this can be seen by using a valued field quantifier free formula defining the graph of $c$. Now we show that $\alpha(K): C(K) \rightarrow \mathbb{Z}$ also has finite image. Namely, if $\alpha(K)$ takes infinitely many values, it must take arbitrarily large values, so there exists an infinite sequence of points $\left(\eta_{n}\right)_{n \in \mathbb{N}}$ in $C(K)$ such that $\alpha(K)\left(\eta_{n}\right)$ is strictly increasing. Since $c(K)$ can take only finitely many values, we may assume that $c\left(\eta_{n}\right)$ takes a constant value $\tilde{c}$ for $n \in \mathbb{N}$. But then the balls $\lambda^{-1} p_{C}^{-1}(K)\left(\eta_{n}\right)$, with $p_{C}: Z_{C, \alpha, \xi, c} \rightarrow C$ for the projection, would have their $t$-adic distance with $\tilde{c}$ go to zero as $n$ increases, which forces $\tilde{c}$ to lie in the ball $X(K)$. Similarly, since all balls $\lambda^{-1} p_{C}^{-1}(K)(\eta)$ are disjoint for different $\eta \in C(K)$, no ball around $\tilde{c}$ can be of the form $\lambda^{-1} p_{C}^{-1}(K)(\eta)$ for $\eta \in C(K)$ and hence the domain of $\lambda$ can not contain $\tilde{c}$ (every ball around $\tilde{c}$ intersects $\lambda^{-1} p_{C}^{-1}(K)\left(\eta_{n}\right)$ for sufficiently large $n)$.

By the discussion in Remark 9.1.6, if for some point $\eta$ in $C(K), c(\eta)$ lies outside $X(K)$, then $C(K)$ is a point and $\alpha(\eta)=\alpha_{1}\left(\eta_{1}(K)\right)$. Reciprocally, if $\alpha(\eta)=$ $\alpha_{1}\left(\eta_{1}(K)\right)$, then $c(\eta)$ lies outside $X(K)$.

We now consider the case of a general $S$. Note that the morphism $\operatorname{Im} c \rightarrow C_{1}$ has globally finite fibers, meaning that the number of fibers is finite and bounded uniformly in $K$, by quantifier elimination of valued field quantifiers.

Denote by $q$ the projection $C \rightarrow C_{1}$. Let us consider the definable subassignment $C_{1}^{\prime}$ of $C_{1}$ consisting of those points $\eta_{1}(K)$ for which there exists a point $\eta$ in $C(K)$ such that $q(\eta)=\eta_{1}$ and $c(\eta)$ lies outside $X(K)$. Denote by $C_{1}^{\prime \prime}$ the complement of $C_{1}^{\prime}$ in $C_{1}$. By additivity we may assume $C_{1}$ is either $C_{1}^{\prime}$ or $C_{1}^{\prime \prime}$. By additivity and a similar finite partitioning argument we may suppose that the number of points in the fibers of $\operatorname{Im} c \rightarrow C_{1}$ is constant and equal to an integer $\delta>0$. We will perform an induction argument on $\delta>0$ where we show that for $\delta=1$ the formula (9.1.1) holds, and that for any presentation $\lambda$ with some $\delta>1$ we can find a presentation $\lambda^{\prime}$ with strictly smaller $\delta$ and such that $\lambda$ and $\lambda^{\prime}$ yield the same result for the left hand side of (9.1.1).

By Remark 9.1.6, one has that $\delta=1$ if and only if $C_{1}=C_{1}^{\prime}$, and then the projection $C \rightarrow h[0,0,0]$ induces an isomorphism $q: C \rightarrow C_{1}$ and $\alpha=\alpha_{1} \circ q$. The statement is clear in this case. Hence we may assume the image of $c$ lies in $X$.

Now suppose that $\delta \geq 2$. For notational simplicity we shall assume again $S=$ $h[0,0,0]$, the general construction being completely similar.

The cell $Z_{C, \alpha, \xi, c}(K)$ induces a partition of $X$ into balls $B_{\eta}$ given by conditions

$$
\operatorname{ord}(x-c(\eta))=\alpha(\eta) \text { and } \overline{\mathrm{ac}}(x-c(\eta))=\xi(\eta),
$$

where $\eta$ runs over $C(K)$. 
For every $K$ such that $C_{1}(K)$ is nonempty, consider the different centers $c_{j}(K)$, $j \in J(K)$ with $J(K)$ finite non empty, where $\operatorname{Im} c(K)=\left\{c_{j}(K)\right\}$. Let us denote by $\beta(K)$ the maximum of ord $\left(c_{i}(K)-c_{j}(K)\right)$ for $i \neq j$ and consider the subset $B(K)$ of $\operatorname{Im} c(K)$ consisting of points $c_{i}(K)$ such that there exists $c_{j}(K)$ with $\operatorname{ord}\left(c_{i}(K)-\right.$ $\left.c_{j}(K)\right)=\beta(K)$. We write $B(K)$ (uniquely) as the disjoint union of subsets $G_{i}$, $i=1, \ldots, d$, such that two points $c_{1}$ and $c_{2}$ of $B(K)$ belong to the same $G_{i}$ if and only if ord $\left(c_{1}-c_{2}\right)=\beta(K)$. For each $G_{i}$ we denote by $B_{i}$ the largest ball containing $G_{i}$ and no point in $\operatorname{Imc}(K) \backslash G_{i}$ and by $\left|G_{i}\right|$ the number of elements in $G_{i}$. For each $i$ consider the barycenter $\tilde{c}_{i}(K):=\left|G_{i}\right|^{-1} \sum_{c_{j} \in G_{i}} c_{j}(K)$. Clearly $\tilde{c}_{i}(K)$ belongs to $B_{i}$ for each $i$.

Each point $\tilde{c}_{i}(K)$ belongs to a unique ball $B_{\eta_{i}}$ for a unique $\eta_{i} \in C(K)$. If $\eta \neq \eta_{i}$ and the ball $B_{\eta} \subset B_{i}$ does occur in the partition we may rewrite it as

$$
\operatorname{ord}\left(x-\tilde{c}_{i}(K)\right)=\alpha(\eta) \quad \text { and } \quad \overline{\operatorname{ac}}\left(x-\tilde{c}_{i}(K)\right)=\xi(\eta)+\overline{\operatorname{ac}}\left(c(\eta)-\tilde{c}_{i}(K)\right) .
$$

Hence we may assume all balls $B_{\eta} \subset B_{i}$ occurring in the partition have center $\tilde{c}_{i}(K)$ except for the ball containing $\tilde{c}_{i}(K)$ which has center $c\left(\eta_{i}\right)$. Now if a ball $B_{\eta}$ occurring in the partition but not contained in $B_{i}$ has a center in $B_{i}$, we can replace that center by $\tilde{c}_{i}(K)$. This shows that we may suppose that each $G_{i}$ consists exactly of the two points $\tilde{c}_{i}(K)$ and $c\left(\eta_{i}\right)$ and that $c\left(\eta_{i}\right)$ is the center of exactly one ball in the decomposition, the one containing $\tilde{c}_{i}(K)$.

When $\delta=2$, one falls back to the computation done in Example 9.1.5 and we can reduce to the case where $\delta=1$ by the computation done in that example. Indeed, with the above notation, the two centers are $\tilde{c}_{1}(K)$ and $c\left(\eta_{1}\right)$ for a unique $\eta_{1} \in C(K)$ and $\operatorname{ord}\left(\tilde{c}_{1}(K)-c\left(\eta_{1}\right)\right)$ is equal to $\max _{\eta \in C(K)}(\alpha(K)(\eta))$.

When $\delta>2$, one reduces to smaller $\delta$ as follows. For every $i, 1 \leq i \leq d$, we denote by $\gamma_{i}(K)$ the supremum of $\operatorname{ord}\left(y-\tilde{c}_{i}(K)\right)$, with $y$ running over $\operatorname{Imc}(K) \backslash B_{i}$ and by $D_{i}$ the smallest ball containing $B_{i}$ and $\tilde{c}_{i}(K)+t^{\gamma_{i}(K)}$, that is, the smallest ball strictly containing $B_{i}$. Of course, one may possibly have $D_{i}=D_{j}$ for some $i \neq j$. We denote by $\gamma(K)$ the supremum of all $\gamma_{i}(K)$, and we denote by $J^{\prime}$ the set of $i$ 's with $\gamma_{i}(K)=\gamma(K)$.

Fix $i$ in $J^{\prime}$. Note that if $B_{j}$ is contained in $D_{i}$, then $j$ lies in $J^{\prime}$. We denote by $\tilde{c}_{i}^{\prime}(K)$ the barycenter of all points in $\operatorname{Imc}(K) \cap D_{i}$. Note that all balls in our cell decomposition that have a center in $D_{i}$ but are not contained in $D_{i}$ may be rewritten so to have center $\tilde{c}_{i}^{\prime}(K)$.

We replace the presentation $\lambda$ by a presentation $\lambda^{\prime}$ obtained in the following way. One keeps all balls $B_{\eta}$ of $\lambda$ not contained in some $D_{i}$ for $i$ in $J^{\prime}$, replacing centers lying in $D_{i}$ by $\tilde{c}_{i}^{\prime}(K)$. Now let us explain how we change the presentation inside a ball $D_{i}, i \in J^{\prime}$. For each $x$ in $\operatorname{Im} c(K) \cap D_{i}$, let $\Gamma_{x}$ be the maximal ball strictly contained in $D_{i}$ and containing $x$. There are finitely many such balls, and we name them $\Gamma_{j, i}$. Note that, by construction, there exist at least two such balls. So, each of the balls $\Gamma_{j, i}$ contains strictly less than $\delta$ points in $\operatorname{Im} c(K)$, so we may apply the induction hypothesis to each of them. That is, we can remove all balls in the presentation $\lambda$ lying in some $\Gamma_{j, i}$, add as new balls the balls $\Gamma_{j, i}$, taking as center 
$\tilde{c}_{i}^{\prime}(K)$, except if $\tilde{c}_{i}^{\prime}(K)$ lies in $\Gamma_{j, i}$, in which case one takes $\tilde{c}_{i}^{\prime}(K)+t^{\gamma(K)}$ as center. One keeps the balls $B_{\eta}$ in the presentation $\lambda$ which are contained in $D_{i}$ but not contained in some $\Gamma_{j, i}$, replacing their center by $\tilde{c}_{i}^{\prime}(K)$, except when $\tilde{c}_{i}^{\prime}(K)$ lies in $B_{\eta}$, in which case one takes $\tilde{c}_{i}^{\prime}(K)+t^{\gamma(K)}$ as center. In this way, one gets a new presentation $\lambda^{\prime}$ with strictly smaller $\delta$, since for each $i$ in $J^{\prime}$, the number of centers lying in $D_{i}$ is at least 3 for $\lambda$ and is equal to 2 for $\lambda^{\prime}$, and the other centers did not change.

Note that the writing of $t^{\gamma(K)}$ involves additional parameters which are harmless: one can always allow parameters of order $\gamma$ to parameterize the center, work relatively over these parameters, and use Remark 9.1.7 to get rid of them after integrating. By the previous application of the induction hypothesis, $\lambda$ and $\lambda^{\prime}$ yield the same result for the left hand side of (9.1.1).

The next Lemma is essential for the proof of Lemma-Definition 9.1.2.

9.1.8. Lemma. - Let $X$ be as in Lemma 9.1.3. Let $\lambda$ be any presentation of $X \backslash Y$ onto a 1-cell $Z_{C, \alpha, \xi, c}$ with basis $C$, order $\alpha$, center $c$, and angular component $\xi$, with $Y \subset X$ a 0 -cell. Write $C \subset S[0, n, r]$ and let $\pi: C \rightarrow S[0,0, r]$ be the projection. Then, in $\mathscr{C}_{+}(S)$,

$$
\mu_{S}\left(\pi_{!}\left(\mathbb{L}^{-\alpha-1}\right)\right)=\mathbb{L}^{-\alpha_{1}-1} \mathbf{1}_{C_{i}} .
$$

9.1.9. Example. - Let $X$ be as in example 9.1.4. A simple presentation $\lambda$ for $X \backslash\{0\}$ is given by $C=h_{\mathbb{G}_{m, k}} \times \mathbb{N}_{0}, c(\eta)=0$, with $\mathbb{N}_{0}=\{a \in \mathbb{Z} \mid a>0\}$,

$$
\operatorname{ord}(x)=\alpha(\eta), \quad \overline{\mathrm{ac}}(x)=\xi(\eta),
$$

where $\eta$ runs over $C$, and $\alpha$ is the projection on $\mathbb{N}_{0}$, and $\xi$ the projection on the multiplicative group of the residue field $h_{\mathbb{G}_{m, k}}$. For this example, one computes that the formula at the end of Lemma 9.1.8 holds. Namely, in $\mathscr{C}_{+}(h[0,0,0])$,

$$
\begin{aligned}
\mu_{h[0,0,0]}\left(\pi_{!}\left(\mathbb{L}^{-\alpha-1}\right)\right) & =\mu_{h[0,0,0]}\left(\left[\mathbb{G}_{m, k}\right] \cdot \mathbb{L}^{-\mathrm{id}-1}\right)=\left[\mathbb{G}_{m, k}\right] \sum_{i>1} \mathbb{L}^{-i} \\
& =\left[\mathbb{G}_{m, k}\right] \frac{\mathbb{L}^{-2}}{\left(1-\mathbb{L}^{-1}\right)}=\frac{\left[\mathbb{G}_{m, k}\right] \mathbb{L}^{-2}}{\mathbb{L}^{-1}(\mathbb{L}-1)}=\mathbb{L}^{-1},
\end{aligned}
$$

since $\left[\mathbb{G}_{m, k}\right] /(\mathbb{L}-1)=1$, where the infinite sum is understood as in section 4.5 and id is the identity function on $\mathbb{N}_{0}$.

Proof of Lemma 9.1.8. - If $Y$ is the empty subassignment, we are in the situation of Lemma 9.1.3 and we are done. So, by a partitioning argument as in the proof of Lemma 9.1.3, we may assume that the preimage of every point $\eta_{1}$ of $C_{1}$ has a nonempty intersection with $Y$. By the discussion in the proof of Lemma 9.1.3 this forces the image of $c$ to be contained in $X$.

As in the proof of Lemma 9.1.3, we shall assume $S=h[0,0,0]$ for notational simplicity, and the constructions being canonical, they will carry over directly to the general relative case by working fiberwise. Fix $K$ with $C_{1}(K)$ nonempty. For every point $x$ in $X(K)$ we denote by $\gamma(x)$ the supremum of $\operatorname{ord}\left(c^{\prime}-x\right)$ for $c^{\prime}$ running 
over all points in $c(C(K))$ different from $x$. Now consider a point $y$ in $Y(K)$ and the ball $B_{y}$ defined by

$$
\operatorname{ord}\left(x-y+t^{\gamma(y)}\right)=\gamma(y) \text { and } \overline{\mathrm{ac}}\left(x-y+t^{\gamma(y)}\right)=1 .
$$

Note that no ball with center $c^{\prime} \neq y$ occurring in the presentation $\lambda$ can be contained in $B_{y}$, since it would necessarily be equal to $B_{y}$, which is impossible. Note that the writing of $t^{\gamma(y)}$ is again harmless: one can always allow parameters of order $\gamma$ to parameterize the center and use Remark 9.1.7 to get rid of them after integrating. Hence $y$ belongs to $c(C(K))$ and all balls occurring in the presentation $\lambda$ that are contained in $B_{y}$ have center $y$. It follows that the restriction of the presentation $\lambda$ to $B_{y}$ is covered by Example 9.1.9. Let us remark that there exists a definable subassignment $Y^{\prime}$ such that, for every $K, Y^{\prime}(K)$ is the union of the balls $B_{y}$ when $y$ runs over $Y(K)$. Hence, if one considers the presentation $\lambda^{\prime}$ of $X$ obtained from the presentation $\lambda$ of $X \backslash Y$ by keeping the balls in $\lambda$ not contained in $Y^{\prime}$, removing the other ones and adding the balls $B_{j}$ as new cells, the statement we have to prove follows from Lemma 9.1.3 applied to $\lambda^{\prime}$. Note that the presentation $\lambda^{\prime}$ exists since one may view the balls $B_{j}$ as parameterized by $Y$.

Proof of 9.1.2 - First we prove independence of $\mu_{S}(\varphi)$ from the choice of $\varphi_{i}, i=$ 0,1 . Actually, $\varphi_{0}$ is uniquely defined. For $\varphi_{1}$, we suppose that there is $\varphi_{1}^{\prime}$ in $\mathscr{C}_{+}(S[1,0,0])$ with $\left[\varphi_{1}\right]=\left[\varphi_{1}^{\prime}\right]$ and we suppose that the cell decomposition $\mathcal{P}_{1}$ is adapted to both $\varphi_{1}$ and $\varphi_{1}^{\prime}$ (see Proposition [7.3.2). For a 1-cell $Z$ in $\mathcal{P}_{1}$ with basis $C$, representation $\lambda: Z \rightarrow Z_{C}$, and projection $p: Z_{C} \rightarrow C$, one has $\psi, \psi^{\prime}$ in $\mathscr{C}_{+}(C)$ satisfying $\lambda^{*} p^{*}(\psi)=\varphi_{1 \mid Z}$ and $\lambda^{*} p^{*}\left(\psi^{\prime}\right)=\varphi_{1 \mid Z}^{\prime}$. Since $\left[\varphi_{1}\right]=\left[\varphi_{1}^{\prime}\right]$ we must have $\psi=\psi^{\prime}$ and hence $\varphi_{1} \mathbf{1}_{Z}=\varphi_{1}^{\prime} \mathbf{1}_{Z}$, by the definitions of 1 -cells and adapted cell decompositions. This shows that there is no dependence on the choice of $\varphi_{1}$ either.

We now prove that $\mu_{S}(\varphi)$ does not depend on the choice of $\mathcal{P}_{0}$. By Proposition 7.3 .2 it is enough to consider a refinement $\mathcal{P}_{0}^{\prime}$ of $\mathcal{P}_{0}$ adapted to $\varphi_{0}$ and to compare $\sum_{Z \in \mathcal{P}_{00}} \mu_{S, Z}\left(\varphi_{0} \mathbf{1}_{Z}\right)$ with $\sum_{Z \in \mathcal{P}_{00}^{\prime}} \mu_{S, Z}\left(\varphi_{0} \mathbf{1}_{Z}\right)$, where $\mathcal{P}_{00}^{\prime}$ is the collection of 0-cells in $\mathcal{P}_{0}^{\prime}$. Clearly, for each 1 -cell $Z$ in $\mathcal{P}_{0}$ (resp. in $\mathcal{P}_{0}^{\prime}$ ) we have $\varphi_{0} \mathbf{1}_{Z}=0$, because $\varphi_{0}$ is of relative dimension 0 and $\mathcal{P}_{0}$ (resp. $\mathcal{P}_{0}^{\prime}$ ) is adapted to $\varphi_{0}$. Note that the union of two 0 -cells is a 0 -cell, and that for two different 0 -cells $Z_{1}, Z_{2}$ in $\mathcal{P}_{00}$ one has

$$
\mu_{S, Z_{1} \cup Z_{2}}\left(\varphi_{0} \mathbf{1}_{Z_{1} \cup Z_{2}}\right)=\mu_{S, Z_{1}}\left(\varphi_{0} \mathbf{1}_{Z_{1}}\right)+\mu_{S, Z_{2}}\left(\varphi_{0} \mathbf{1}_{Z_{2}}\right) .
$$

Let $Z$ be the union of all 0 -cells in $\mathcal{P}_{00}$ and $Z^{\prime}$ be the union of all 0 -cells in $\mathcal{P}_{00}^{\prime}$. Then $Z$ and $Z^{\prime}$ are 0 -cells. Since $\mathcal{P}_{0}^{\prime}$ is a refinement of $\mathcal{P}_{0}$, it follows that $Z \subset Z^{\prime}$ and that $Z^{\prime} \backslash Z$ is also a 0 -cell. We also have $\varphi_{0} \mathbf{1}_{Z^{\prime} \backslash Z}=0$, since $\mathcal{P}_{0}$ and $\mathcal{P}_{0}^{\prime}$ are adapted to $\varphi_{0}$. One computes

$$
\begin{aligned}
\mu_{S, Z^{\prime}}\left(\varphi_{0} \mathbf{1}_{Z^{\prime}}\right) & =\mu_{S, Z}\left(\varphi_{0} \mathbf{1}_{Z}\right)+\mu_{S, Z^{\prime} \backslash Z}\left(\varphi_{0} \mathbf{1}_{Z^{\prime} \backslash Z}\right) \\
& =\mu_{S, Z}\left(\varphi_{0} \mathbf{1}_{Z}\right)
\end{aligned}
$$

which proves that $\mu_{S}(\varphi)$ is independent of the choice of $\mathcal{P}_{0}$. 
Now let us prove that $\mu_{S}(\varphi)$ is independent of the choice of $\mathcal{P}_{1}$. By Proposition 7.3.2 it is enough to compare two cell decompositions $\mathcal{P}_{1}$ and $\mathcal{P}_{1}^{\prime}$ of $S[1,0,0]$ adapted to $\varphi_{1}$ such that $\mathcal{P}_{1}^{\prime}$ is a refinement of $\mathcal{P}_{1}$. Fix a 1 -cell $Z$ in $\mathcal{P}_{1}$. Note that the union of two disjoint 1-cells is a single 1-cell. Similarly, the union of two disjoint 0-cells is a single 0 -cell. For the disjoint union of two 1 -cells $Z_{1}, Z_{2}$, adapted to $\varphi$, one has clearly

$$
\mu_{S, Z_{1} \cup Z_{2}}\left(\varphi_{1} \mathbf{1}_{Z_{1} \cup Z_{2}}\right)=\mu_{S, Z_{1}}\left(\varphi_{1} \mathbf{1}_{Z_{1}}\right)+\mu_{S, Z_{2}}\left(\varphi_{1} \mathbf{1}_{Z_{2}}\right) .
$$

Also, a 0-cell cannot contain a 1-cell. Hence, we may suppose that $Z^{\prime} \subset Z$ and that $Z \backslash Z^{\prime}$ is a 0 -cell, with $Z^{\prime}$ in $\mathcal{P}_{1}^{\prime}$. Let $\lambda: Z \rightarrow Z_{C}=Z_{C, \alpha, \xi, c}$ be a presentation of $Z$, and let $\lambda^{\prime}: Z^{\prime} \rightarrow Z_{C^{\prime}}^{\prime}=Z_{C^{\prime}, \alpha^{\prime}, \xi^{\prime}, c^{\prime}}^{\prime}$ be a presentation of $Z^{\prime}$. Write $p: Z_{C} \rightarrow C$ and $p^{\prime}: Z_{C^{\prime}}^{\prime} \rightarrow C^{\prime}$ for the projections. By a fiber product argument we may suppose that $\lambda=\mathrm{id}: Z \rightarrow Z=Z_{C}$. Let $Z_{1}^{\prime}$ be the definable subassignment of $Z^{\prime}$ determined by

$$
x \in Z^{\prime} \wedge \alpha \circ p(x)=\alpha^{\prime} \circ p^{\prime} \circ \lambda^{\prime}(x),
$$

and set $Z_{1}:=Z_{1}^{\prime}, Z_{2}:=Z \backslash Z_{1}, Z_{2}^{\prime}:=Z^{\prime} \backslash Z_{1}^{\prime}$.

Then, by Proposition 7.2.5, $Z_{i}$ and $Z_{i}^{\prime}, i=1,2$, are either empty or 1-cells. Also, for $x$ in $Z_{2}^{\prime}$,

$$
\alpha \circ p(x)<\alpha^{\prime} \circ p^{\prime} \circ \lambda^{\prime}(x)
$$

by the non archimedean property, since $Z^{\prime} \subset Z$.

Since the equalities $\mu_{S, Z_{i}}\left(\varphi \mathbf{1}_{Z_{i}}\right)=\mu_{S, Z_{i}^{\prime}}\left(\varphi \mathbf{1}_{Z_{i}^{\prime}}\right)$ for $i=1,2$ imply that $\mu_{S, Z}\left(\varphi \mathbf{1}_{Z}\right)=$ $\mu_{S, Z^{\prime}}\left(\varphi \mathbf{1}_{Z^{\prime}}\right)$ by the above discussion, it is enough to prove the following claim.

9.1.10. Claim. - One has $\mu_{S, Z_{2}}\left(\varphi \mathbf{1}_{Z_{2}}\right)=\mu_{S, Z_{2}^{\prime}}\left(\varphi \mathbf{1}_{Z_{2}^{\prime}}\right)$.

For the proof of the claim, we may suppose that $Z=Z_{2}$ and $Z^{\prime}=Z_{2}^{\prime}$. It is enough to show that

$$
\mu_{S, Z^{\prime}}\left(\mathbf{1}_{Z^{\prime}}\right)=\mathbb{L}^{\alpha-1} \mathbf{1}_{C}
$$

holds in $\mathscr{C}_{+}(S)$, which follows from Lemma 9.1.8,

9.2. Direct image under the projection $S[1,0,0] \rightarrow S$. - Let $S$ be in $\operatorname{Def}_{k}$ and write $\pi: S[1,0,0] \rightarrow S$ for the projection. Let $\varphi$ be in $C_{+}(S[1,0,0])$.

We first suppose that $\varphi$ is in $C_{+}^{d}(S[1,0,0])$ for some $d$. Fix $\hat{\varphi}$ in $\mathscr{C}_{+}^{\leq d}(S[1,0,0])$ such that $\varphi$ is the class of $\hat{\varphi}$. Let $\mathcal{P}$ be a cell decomposition of $S[1,0,0]$ adapted to $\hat{\varphi}$ as in Theorem 7.2.1 and set $\mathcal{P}_{i}=\{Z \in \mathcal{P} \mid Z$ is a $i$-cell $\}$ for $i=0,1$.

Fix $Z_{i}$ in $\mathcal{P}_{i}, i=0,1$. The cell $Z_{0}$ has a presentation

$$
\lambda_{0}: Z_{0} \rightarrow Z_{C_{0}}=Z_{C_{0}, c_{0}} \subset S\left[0, s_{0}, 0\right],
$$

for some $s_{0} \geq 0$ and some definable morphism $c_{0}$. There is a unique $\psi_{0}$ in $\mathscr{C}_{+}\left(C_{0}\right)$ such that

$$
\hat{\varphi}_{\mid Z_{0}}=\lambda_{0}^{*} p_{0}^{*}\left(\psi_{0}\right)
$$

where $p_{0}$ is the projection $p_{0}: Z_{C_{0}} \rightarrow C_{0}$. We write $j_{0}$ for the inclusion $j_{0}: C_{0} \rightarrow$ $S\left[0, s_{0}, 0\right]$, and $\pi_{0}$ for the projection $S\left[0, s_{0}, 0\right] \rightarrow S$. Denote by $\gamma: C_{0} \rightarrow \mathbb{Z}$ the definable morphism $y \mapsto\left(\operatorname{ordjac} p_{0}\right) \circ p_{0}^{-1}$, where ordjac is defined as in section 8.4. 
9.2.1. Lemma-Definition. - The following definitions are independent of the choice of $\lambda_{0}$, where we use the above notation. We define

$$
\pi_{! Z_{0}, d}\left(\hat{\varphi} \mathbf{1}_{Z_{0}}\right)
$$

as the image of the constructible function $\pi_{0 !}\left(j_{0 !}\left(\psi_{0} \mathbb{L}^{\gamma}\right)\right)$ in $\mathscr{C}_{+}^{\leq d}(S)$ under the natural morphism $\mathscr{C}_{+}^{\leq d}(S) \rightarrow C_{+}^{d}(S)$. Here, $j_{0 \text { ! }}$ and $\pi_{0 \text { ! }}$ are as in sections 5.5 and 5.6. respectively.

Also, we say $\hat{\varphi} \mathbf{1}_{Z_{1}}$ is $S$-integrable along $Z_{1}$ if $\hat{\varphi} \mathbf{1}_{Z_{1}}$ is $S$-integrable along $Z_{1}$ as in 9.1.1. If this is the case,

$$
\mu_{S, Z_{1}}\left(\hat{\varphi} \mathbf{1}_{Z_{1}}\right)
$$

as defined in Lemma-Definition 9.1.1 lies in $\mathscr{C}_{+}^{\leq d-1}(S)$ and we define

$$
\pi_{! Z_{1}, d}\left(\hat{\varphi} \mathbf{1}_{Z_{1}}\right)
$$

as the image of $\mu_{S, Z_{1}}\left(\hat{\varphi} \mathbf{1}_{Z_{1}}\right)$ under the natural morphism $\mathscr{C}_{+}^{\leq d-1}(S) \rightarrow C_{+}^{d-1}(S)$.

9.2.2. Lemma-Definition. - The following definitions are independent of the choice of $\mathcal{P}$ and $\hat{\varphi}$, where we use the above notation. We say $\varphi$ is $S$-integrable if $\hat{\varphi} \mathbf{1}_{Z}$ is $S$-integrable along $Z$ for each $Z$ in $\mathcal{P}_{1}$. If this is the case we define $\pi_{!}(\varphi)$ in $\mathscr{C}_{+}(S)$ as

$$
\pi_{!}(\varphi):=\sum_{Z \in \mathcal{P}} \pi_{! Z, d}\left(\hat{\varphi} \mathbf{1}_{Z}\right)
$$

where $\pi_{! Z, d}\left(\hat{\varphi} \mathbf{1}_{Z}\right)$ is defined as in 9.2.1.

Finally we take a general $\varphi$ in $C_{+}(S[1,0,0])$ and we write $\varphi=\sum_{i} \varphi_{i}$ with $\varphi_{i}$ in $C_{+}^{i}(S[1,0,0])$. We set

$$
\pi_{!}(\varphi):=\sum_{i} \pi_{!}\left(\varphi_{i}\right)
$$

where each $\pi_{!}\left(\varphi_{i}\right)$ is defined as in 9.2.2. By the above discussion this is independent of the choices.

Proof of 9.2.1 - Let $\lambda_{0}^{\prime}: Z_{0} \rightarrow Z_{C_{0}^{\prime}, c_{0}^{\prime}}^{\prime}$ be a different presentation of $Z_{0}$. Since $Z_{0}$ is a 0-cell, clearly there is a definable isomorphism $g: C_{0} \rightarrow C_{0}^{\prime}$ compatible with the maps $Z_{0} \rightarrow C_{0}$ and $Z_{0} \rightarrow C_{0}^{\prime}$. By Proposition 8.4.1 and the definition of ordjac, $\gamma=\left(\operatorname{ordjac} p_{0}^{\prime}\right) \circ p_{0}^{\prime-1} \circ g$, where $p_{0}^{\prime}$ denotes the projection $p_{0}^{\prime}: Z_{C_{0}^{\prime}} \rightarrow C_{0}^{\prime}$. Hence, independence from the choice of $\lambda_{0}$ follows by functorial properties of the pullback. Since $Z_{0}$ is a 0 -cell, one has by Proposition 3.4.1 that $\operatorname{Kdim} X=\operatorname{Kdim} \pi(X)$ for each $X \subset Z_{0}$, where still $\pi: S[1,0,0] \rightarrow S$ is the projection. Hence, it follows that $\pi_{0 !}\left(j_{0 !}\left(\psi_{0} \mathbb{L}^{\gamma}\right)\right)$ is in $\mathscr{C}_{+}^{\leq d}(S)$. Similarly it follows that $\mu_{S, Z_{1}}\left(\mathbf{1}_{Z_{1}} \hat{\varphi}\right)$ is in $\mathscr{C}_{+}^{\leq d-1}(S)$ by Proposition 3.4.1.

Proof of 9.2.2 - First we prove independence of $\pi_{!}(\varphi)$ from the choice of $\hat{\varphi}$. Suppose that there is $\hat{\varphi}^{\prime}$ in $\mathscr{C}_{+}^{\leq d}(S[1,0,0])$ whose class in $C_{+}^{d}(S[1,0,0])$ is $\varphi$. Then there exist $\varepsilon$ and $\varepsilon^{\prime}$ in $\mathscr{C}_{+}^{\leq d-1}(S[1,0,0])$ such that $\hat{\varphi}+\varepsilon=\hat{\varphi}^{\prime}+\varepsilon^{\prime}$. We may suppose that the 
cell decomposition $\mathcal{P}$ is adapted to $\hat{\varphi}, \hat{\varphi}^{\prime}, \varepsilon$, and $\varepsilon^{\prime}$. By dimensional considerations similar to the ones used in the proof of Lemma-Definition 9.2.1, we get that

$$
\sum_{Z \in \mathcal{P}} \pi_{! Z, d}\left(\varepsilon \mathbf{1}_{Z}\right)=\sum_{Z \in \mathcal{P}} \pi_{! Z, d}\left(\varepsilon^{\prime} \mathbf{1}_{Z}\right)=0
$$

Thus,

$$
\sum_{Z \in \mathcal{P}} \pi_{! Z, d}\left(\hat{\varphi} \mathbf{1}_{Z}\right)=\sum_{Z \in \mathcal{P}} \pi_{! Z, d}\left(\hat{\varphi}^{\prime} \mathbf{1}_{Z}\right)
$$

by the additivity of $\pi_{! Z, d}$, which shows the independence from the choice of $\hat{\varphi}$.

We shall now prove that $\pi_{!}(\varphi)$ is independent of the choice of $\mathcal{P}$. By Proposition 7.3.2 it is enough to compare two cell decompositions $\mathcal{P}$ and $\mathcal{P}^{\prime}$ of $S[1,0,0]$ adapted to $\hat{\varphi}$ such that $\mathcal{P}^{\prime}$ is a refinement of $\mathcal{P}$. Similarly as in the proof of Lemma-Definition 9.1.2, we can fix a 1-cell $Z_{1}$ in $\mathcal{P}$ and we may suppose that $Z_{1}^{\prime}$ in $\mathcal{P}^{\prime}$ is a 1-cell such that $Z_{0}^{\prime}:=Z_{1} \backslash Z_{1}^{\prime}$ is a 0 -cell in $\mathcal{P}^{\prime}$. By dimensional considerations as in the proof of Lemma-Definition 9.2.1, we find that $\pi_{! Z_{0}^{\prime}, d}\left(\hat{\varphi} \mathbf{1}_{Z_{0}^{\prime}}\right)=0$. Hence, we only have to show that

$$
\pi_{! Z_{1}, d}\left(\hat{\varphi} \mathbf{1}_{Z_{1}}\right)=\pi_{! Z_{1}^{\prime}, d}\left(\hat{\varphi} \mathbf{1}_{Z_{1}^{\prime}}\right)
$$

and

$$
\sum_{j} \pi_{! Z_{j}, d}\left(\hat{\varphi} \mathbf{1}_{Z_{j}}\right)=\sum_{i} \pi_{! Z_{i}^{\prime}, d}\left(\hat{\varphi} \mathbf{1}_{Z_{j}^{\prime}}\right)
$$

where the sum on the left, resp. right, hand side is over all 0 -cells in $\mathcal{P}$, resp. $\mathcal{P}^{\prime}$. The first equality follows from Claim 9.1.10 in the same way as this claim is used in the proof of Lemma-Definition 9.1.2. The second equality follows in a way similar to the statement for 0-cells in the proof of Lemma-Definition 9.1.2.

\subsection{Basic properties. -}

9.3.1. Proposition. - Let $S$ be in $\operatorname{Def}_{k}$ and $f, g$ both in $C_{+}(S[1,0,0] \rightarrow S)$ or both in $C_{+}(S[1,0,0])$. If $g \geq f$ and $g$ is $S$-integrable, then $f$ is $S$-integrable.

Proof. - This follows immediately from Proposition 5.8.3 and the definition of integrability in 9.1 .2 by taking a cell decomposition adapted to $f$ and $g$ which exists by Proposition 7.3.2.

\subsubsection{Proposition (Change of variable in relative dimension 1)}

Let $X$ and $Y$ be definable subassignments of $S[1,0,0]$ for some $S$ in $\operatorname{Def}_{k}$ and let $f: X \rightarrow Y$ be a definable isomorphism over $S$. Suppose that $X$ and $Y$ are equidimensional of relative dimension 1 relative to the projection to $S$. Let $\varphi$ be in $C_{+}^{1}(Y \rightarrow S)$. We use $\operatorname{ordjac}_{S} f$ as defined in section 8.5. Then, $\varphi$ is $S$-integrable if and only if $\mathbb{L}^{-\operatorname{ordjac}_{S} f} f^{*}(\varphi)$ is $S$-integrable and if this is the case then

$$
\mu_{S}(\varphi)=\mu_{S}\left(\mathbb{L}^{-\operatorname{ordjac}_{S} f} f^{*}(\varphi)\right)
$$

holds in $\mathscr{C}_{+}(S)$. 
Proof. - This follows from Theorem 7.5.3 and its corollary. Note that the morphisms $\beta_{i} \circ p_{i} \circ \lambda_{i}$ on the 1-cells $Z_{i}$, defined in Theorem 7.5.3 (3), have the same class as $\operatorname{ordjac}_{S} f_{\mid Z_{i}}$.

\section{II CONSTRUCTION OF THE GENERAL MOTIVIC MEASURE}

\section{Statement of the main result}

10.1. Integration. - In this section, and until section 15, all definable subassignments will belong to $\operatorname{Def}_{k}$. In particular they will be affine. To be able to integrate positive motivic constructible Functions, we have to define integrable positive Functions. These, and more generally $S$-integrable positive Functions, will be defined inductively, as follows:

10.1.1. Theorem. - Let $S$ be in $\operatorname{Def}_{k}$. There is a unique functor from the category $\operatorname{Def}_{S}$ to the category of abelian semigroups, $Z \mapsto \mathrm{I}_{S} C_{+}(Z)$, assigning to every morphism $f: Z \rightarrow Y$ in $\operatorname{Def}_{S}$ a morphism $f_{!}: \mathrm{I}_{S} C_{+}(Z) \rightarrow \mathrm{I}_{S} C_{+}(Y)$ and satisfying the following axioms:

A0 (Functoriality):

(a) For every composable morphisms $f$ and $g$ in $\operatorname{Def}_{S},(f \circ g)_{!}=f_{!} \circ g_{!}$. In particular, $\mathrm{id}_{!}=\mathrm{id}$.

(b) Let $\lambda: S \rightarrow S^{\prime}$ be a morphism in Def $_{k}$ and denote by $\lambda_{+}:$Def $_{S} \rightarrow$ Def $_{S^{\prime}}$ the functor induced by composition with $\lambda$, we have the inclusion $\mathrm{I}_{S^{\prime}} C_{+}\left(\lambda_{+}(Z)\right) \subset \mathrm{I}_{S} C_{+}(Z)$ for $Z$ in $\operatorname{Def}_{S}$, and for $\varphi$ in $\mathrm{I}_{S^{\prime}} C_{+}\left(\lambda_{+}(Z)\right)$, $f_{!}(\varphi)$ is the same Function computed in $\mathrm{I}_{S}$ or in $\mathrm{I}_{S^{\prime}}$.

(c) If $f: X \rightarrow Y$ is a morphism in Def $_{S}$, a positive constructible Function $\varphi$ on $X$ belongs to $\mathrm{I}_{S} C_{+}(X)$ if and only if $\varphi$ belongs to $\mathrm{I}_{Y} C_{+}(X)$ and $f_{!}(\varphi)$ belongs to $\mathrm{I}_{S} C_{+}(Y)$.

A1 (Integrability):

(a) For every $Z$ in $\operatorname{Def}_{S}, \mathrm{I}_{S} C_{+}(Z)$ is a graded subsemigroup of $C_{+}(Z)$.

(b) $\mathrm{I}_{S} C_{+}(S)=C_{+}(S)$.

A2 (Additivity): Let $Z$ be a definable subassignment in $\operatorname{Def}_{S}$. Assume $Z$ is the disjoint union of two definable subassignments $Z_{1}$ and $Z_{2}$. Then, for every morphism $f: Z \rightarrow Y$ in $\operatorname{Def}_{S}$, the isomorphism $C_{+}(Z) \simeq C_{+}\left(Z_{1}\right) \oplus C_{+}\left(Z_{2}\right)$ induces an isomorphism $\mathrm{I}_{S} C_{+}(Z) \simeq \mathrm{I}_{S} C_{+}\left(Z_{1}\right) \oplus \mathrm{I}_{S} C_{+}\left(Z_{2}\right)$ under which we have $f_{!}=f_{\mid Z_{1} !} \oplus f_{\mid Z_{2} !}$.

A3 (Projection formula): For every morphism $f: Z \rightarrow Y$ in $\operatorname{Def}_{S}$, and every $\alpha$ in $\mathscr{C}_{+}(Y)$ and $\beta$ in $\mathrm{I}_{S} C_{+}(Z), \alpha f_{!}(\beta)$ belongs to $\mathrm{I}_{S} C_{+}(Y)$ if and only if $f^{*}(\alpha) \beta$ is in $\mathrm{I}_{S} C_{+}(Z)$. If these conditions are verified, then $f_{!}\left(f^{*}(\alpha) \beta\right)=\alpha f_{!}(\beta)$. 
A4 (Inclusions): If $i: Z \hookrightarrow Z^{\prime}$ be the inclusion between two definable subassignments of some object in $\operatorname{Def}_{S}$, for every $\varphi$ in $\mathscr{C}_{+}(Z),[\varphi]$ lies in $\mathrm{I}_{S} C_{+}(Z)$ if and only if $\left[i_{!}(\varphi)\right]$ belongs to $\mathrm{I}_{S} C_{+}\left(Z^{\prime}\right)$, with $i_{!}$defined as in 5.5. If this is the case, then $i_{!}([\varphi])=\left[i_{!}(\varphi)\right]$.

A5 (Projection along $k$-variables): Let $Y$ be in $\operatorname{Def}_{S}$. Consider the projection $\pi: Z=Y[0, n, 0] \rightarrow Y$. Let $\varphi$ be in $\mathscr{C}_{+}(Z)$. Then $[\varphi]$ belongs to $\mathrm{I}_{S} C_{+}(Z)$ if and only if $\left[\pi_{!}(\varphi)\right]$ belongs to $\mathrm{I}_{S} C_{+}(Y), \pi_{!}$being defined as in 5.6. Furthermore, when this holds, $\pi_{!}([\varphi])=\left[\pi_{!}(\varphi)\right]$.

A6 (Projection along $\mathbb{Z}$-variables): Let $Y$ be in $\operatorname{Def}_{S}$. Consider the projection $\pi: Z=Y[0,0, r] \rightarrow Y$. Take $\varphi$ in $\mathscr{C}_{+}(Z)$. Then $[\varphi]$ belongs to $\mathrm{I}_{S} C_{+}(Z)$ if and only if there is a function $\varphi^{\prime}$ in $\mathscr{C}_{+}(Z)$ with $\left[\varphi^{\prime}\right]=[\varphi]$ such that $\varphi^{\prime}$ is $\pi$-integrable in the sense of 5.7 and $\left[\mu_{S}\left(\varphi^{\prime}\right)\right]$ belongs to $\mathrm{I}_{S} C(Y)$. Furthermore, when this holds, $\pi_{!}([\varphi])=\left[\mu_{S}\left(\varphi^{\prime}\right)\right]$.

A7 (Relative annuli): Let $Y$ be in $\operatorname{Def}_{S}$ and consider definable morphisms $\alpha: Y \rightarrow \mathbb{Z}, \xi: Y \rightarrow h_{\mathbb{G}_{m, k}}$, with $\mathbb{G}_{m, k}$ the multiplicative group $\mathbb{A}_{k}^{1} \backslash\{0\}$, and $c: Y \rightarrow h_{\mathbb{A}_{k((t))}^{1}}$. Then, if $Z$ is the definable subassignment of $Y[1,0,0]$ defined by $\operatorname{ord}(z-c(y))=\alpha(y)$ and $\overline{\mathrm{ac}}(z-c(y))=\xi(y)$, and $f: Z \rightarrow Y$ is the morphism induced by the projection $Y \times h_{\mathbb{A}_{k(t))}^{1}} \rightarrow Y,\left[\mathbf{1}_{Z}\right]$ is in $\mathrm{I}_{S} C_{+}(Z)$ if and only $\mathbb{L}^{-\alpha-1}\left[\mathbf{1}_{Y}\right]$ belongs to $\mathrm{I}_{S} C_{+}(Y)$, and, if this is the case, then

$$
f_{!}\left(\left[\mathbf{1}_{Z}\right]\right)=\mathbb{L}^{-\alpha-1}\left[\mathbf{1}_{Y}\right] \text {. }
$$

A8 (Graphs): Let $Y$ be in $\operatorname{Def}_{S}$ and consider a definable morphism $c: Y \rightarrow$ $h_{\mathbb{A}_{k((t))}^{1}}$. If $Z$ is the definable subassignment of $Y[1,0,0]$ defined by $z-c(y)=0$ and $p: Z \rightarrow Y$ is the projection, $\left[\mathbf{1}_{Z}\right]$ is in $\mathrm{I}_{S} C_{+}(Z)$ if and only $\mathbb{L}^{\text {(ordjacp)op } p^{-1}}$ belongs to $\mathrm{I}_{S} C_{+}(Y)$, and, if this is the case, then

$$
f_{!}\left(\left[\mathbf{1}_{Z}\right]\right)=\mathbb{L}^{(\operatorname{ordjac} p) \circ p^{-1}} .
$$

For $f: X \rightarrow S$ a morphism, elements of $\mathrm{I}_{S} C_{+}(X)$ shall be called $S$-integrable positive Functions (or $f$-integrable positive Functions).

10.1.2. Remark. - Axiom A8 is a special case of Theorem 12.1.1 so Theorem 12.1.1 could replace A8 as an axiom.

10.1.3. Remark. - In general $f_{!}$is a morphism of abelian semigroups but not of graded semigroups. There is a shift by the relative $K$-dimension, as, for instance, in axiom $\mathrm{A} 7$.

10.2. Motivic measure. - When $f: Z \rightarrow h_{\mathrm{Spec} k}$ is the projection onto the final subassignment, we write $\mathrm{I}_{+}(Z)$ for $\mathrm{I}_{h_{\mathrm{Spec} k}} C_{+}(Z)$. For $\varphi$ in $\mathrm{I}_{+}(Z)$, we define the motivic measure $\mu(\varphi)$ as $f_{!}(\varphi)$ in $I_{+}(\operatorname{Spec} k)=S K_{0}\left(\operatorname{RDef}_{h_{\text {Spec } k}}\right) \otimes_{\mathbb{N}[\mathbb{L}-1]} \mathbb{A}_{+}$. We shall write also $\mu(Z)$ for $\mu\left(\left[\mathbf{1}_{Z}\right]\right)$, when $Z$ is a definable subassignment of $h[m, n, 0]$ such that $\left[\mathbf{1}_{Z}\right]$ is integrable. By Proposition 12.2 .2 this happens as soon as $Z$ is bounded in the sense of 12.2 . 


\section{Proof of Theorem 10.1 .1}

Recall that in this section, and until section 15, all definable subassignments belong to $\operatorname{Def}_{k}$, so, in particular, they are affine.

11.1. Uniqueness. - Using A0, it is enough to show, for every $f: X \rightarrow S$, the uniqueness of $\mathrm{I}_{S} C_{+}(X)$ and of $f_{!}: \mathrm{I}_{S} C_{+}(X) \rightarrow \mathrm{I}_{S} C_{+}(S)=C_{+}(S)$. We consider first the case of a projection $\pi: S \times Y \rightarrow S$ with $Y$ definable subassignment of some $h[m, n, r]$. We may assume $Y=h[m, n, r]$. Indeed, $\pi$ may be factorized as

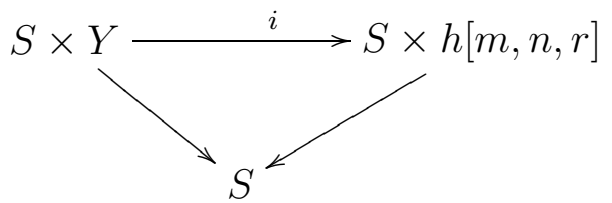

with $i$ the inclusion, so we are done by A0 and A4. The case where $m=0$ is dealed with by using A5, A6, and A0. Let us consider now the case $m=1$ and take $\varphi$ in $\mathscr{C}_{+}(S[1,0,0])$. By Theorem 7.2 .1 , there exists a cell decomposition $\mathcal{Z}$ of $S[1,0,0]$ adapted to $\varphi$, that is, a finite partition of $S[1,0,0]$ into cells $Z_{i}$ with presentation $\left(\lambda_{i}, Z_{C_{i}}\right)$, such that $\varphi_{\mid Z_{i}}=\tilde{\lambda}_{i}^{*} p_{i}^{*}\left(\psi_{i}\right)$, with $\psi_{i}$ in $\mathscr{C}\left(C_{i}\right)$ and $p_{i}: Z_{C_{i}} \rightarrow C_{i}$ the projection. Furthermore, maybe after applying again Theorem 7.2.1 and taking a refinement of $\mathcal{Z}$, we may assume the following condition:

$\varphi_{\mid Z_{i}}$ is either zero or has the same $K$-dimension as $Z_{i}$ for every $i$.

Using A2 and A4 we may reduce to the case of the projection $f: Z \rightarrow S$ of a cell $Z$ of $K$-dimension $d$ in $S[1,0,0]$ with presentation $\left(\lambda, Z_{C}\right)$ and a function $\varphi$ in $\mathscr{C}_{+}(Z)$ of $K$-dimension $d$ such that $\varphi=\tilde{\lambda}^{*} p^{*}(\psi)$, with $\psi$ in $\mathscr{C}_{+}(C)$ and $p: Z_{C} \rightarrow C$ the projection. We have to decide when $[\varphi]$ belongs to $\mathrm{I}_{S} C_{+}(Z)$ and if it is the case to compute the value of $f_{!}([\varphi])$. Let us denote by $\tilde{\pi}: Z_{C} \rightarrow Z$ the restriction of the projection on the $S[1,0,0]$-factor to $Z_{C}$. The morphism $\tilde{\pi}$ is the inverse of $\tilde{\lambda}$. Since $\tilde{\pi} \circ \tilde{\lambda}=\mathrm{id}$, it follows from A0 that $\tilde{\pi}_{!}$and $\tilde{\lambda}_{!}$are mutually inverse. It follows from A4, A5 and A6 that $\tilde{\pi}_{!}\left(\left[\mathbf{1}_{Z_{C}}\right]\right)=\left[\mathbf{1}_{Z}\right]$, hence $\tilde{\lambda}_{!}\left(\left[\mathbf{1}_{Z}\right]\right)=\left[\mathbf{1}_{Z_{C}}\right]$. So, by using the projection formula A3, one gets that $[\varphi]$ belongs to $\mathrm{I}_{S} C_{+}(Z)$ if and only if $p^{*} \psi\left[\mathbf{1}_{Z_{C}}\right]$ belongs to $\mathrm{I}_{S} C_{+}\left(Z_{C}\right)$. By A0, A1(b) and A3 this is equivalent to the condition that $\psi p_{!}\left(\left[\mathbf{1}_{Z_{C}}\right]\right)$ belongs to $\mathrm{I}_{S} C_{+}(C)$, which amounts to the case $m=0$ already considered. Now if $[\varphi]$ belongs to $\mathrm{I}_{S} C_{+}(Z)$, plugging in axioms $\mathrm{A} 7$ or $\mathrm{A} 8$ depending on the type of the cell $Z_{C}$, completely determines the value of $f_{!}([\varphi])$ : it should be equal to $h_{!}\left(\psi p_{!}\left(\left[\mathbf{1}_{Z_{C}}\right]\right)\right.$, with $h$ the canonical morphism $C \rightarrow S$.

Now consider the case of a general morphism $f: X \rightarrow S$. We factor it as $f=\pi \circ \gamma_{f}$, with $\gamma_{f}: X \rightarrow X \times S$ the graph morphism and $\pi: X \times S \rightarrow S$ the projection. We consider also the projection $p: X \times S \rightarrow X$. Since $p \circ \gamma_{f}=\mathrm{id}$, it follows from $\mathrm{A} 0$ and $\mathrm{A} 1$ that $\mathrm{I}_{X \times S} C_{+}(X)=C_{+}(X)$. Hence a Function $\phi$ in $C_{+}(X)$ will belong to $\mathrm{I}_{S} C_{+}(X)$ if and only $\gamma_{f !}(\phi)$ belongs to $\mathrm{I}_{S} C_{+}(X \times S)$ and then $f_{!}(\phi)=\pi_{!}\left(\gamma_{f !}(\phi)\right)$. Hence we are left with showing the uniqueness of $\gamma_{f !}$. It is enough to show that $\gamma_{f !}\left(\varphi\left[\mathbf{1}_{X}\right]\right)$ is uniquely determined for $\varphi$ in $\mathscr{C}_{+}(X)$, since one 
can always reduce to that case replacing $X$ by some subassignment and using A4 and A2. Let us denote by $\Gamma_{f}$ the graph of $f$. It follows from the previous discussion of projections that $p_{!}\left[\mathbf{1}_{\Gamma_{f}}\right]$ should be of the form $\mathbb{L}^{\alpha}\left[\mathbf{1}_{X}\right]$ for some definable function $\alpha$ on $X$. Since $\mathbb{L}^{\alpha}=\gamma_{f}^{*} \mathbb{L}^{\alpha \circ p}$, we get

$$
\left[\mathbf{1}_{\Gamma_{f}}\right]=\gamma_{f !} p_{!}\left(\left[\mathbf{1}_{\Gamma_{f}}\right]\right)=\gamma_{f !}\left(\gamma_{f}^{*} \mathbb{L}^{\alpha \circ p}\left[\mathbf{1}_{X}\right]\right)=\mathbb{L}^{\alpha \circ p} \gamma_{f !}\left(\left[\mathbf{1}_{X}\right]\right),
$$

by using functoriality and the projection formula hence $\gamma_{f !}\left(\left[\mathbf{1}_{X}\right]\right)$ should be equal to $L^{-\alpha \circ p}\left[\mathbf{1}_{\Gamma_{f}}\right]$. Since $\varphi=\gamma_{f}^{*} p^{*} \varphi$, it follows again from the projection formula that $\gamma_{f !}\left(\varphi\left[\mathbf{1}_{X}\right]\right)$ is uniquely determined.

11.2. Projections. - We will now construct $\mathrm{I}_{S} C_{+}(S \times Y)$ and $\pi_{\text {! when }} \pi$ is the projection $S \times Y \rightarrow S$. We start by assuming $Y=h[m, n, r]$, so that $S \times Y=$ $S[m, n, r]$.

When $m=n=r=0$ we set $\mathrm{I}_{S} C_{+}(S)=C_{+}(S)$ and $\pi_{!}=\mathrm{id}$.

More generally, we set $\mathrm{I}_{S} C_{+}(S[0, n, 0])=C_{+}(S[0, n, 0])$ and define

$$
\pi_{!}: \mathrm{I}_{S} C_{+}(S[0, n, 0]) \rightarrow C_{+}(S)
$$

by $\pi_{!}([\varphi])=\left[\pi_{!}(\varphi)\right]$, for $\varphi$ in $\mathscr{C}_{+}(S[0, n, 0])$ of $K$-dimension $d, \pi_{!}: \mathscr{C}_{+}(S[0, n, 0]) \rightarrow$ $\mathscr{C}_{+}(S)$ being defined as in 5.6 .

Similarly, when $m=n=0$, we define $\mathrm{I}_{S} C_{+}(S[0,0, r])$ as dictated by A6. That is, for $\varphi$ in $C_{+}(Z)$, we shall say $\varphi$ belongs to $\mathrm{I}_{S} C_{+}(S[0,0, r])$ if and only if there is a function $\varphi^{\prime}$ in $\mathscr{C}_{+}(S[0,0, r])$ with $\left[\varphi^{\prime}\right]=\varphi$ such that $\varphi^{\prime}$ is $S$-integrable in the sense of 5.7, and we set $\pi_{!}(\varphi)=\left[\mu_{S}\left(\varphi^{\prime}\right)\right]$. Clearly this definition is independent of the choice of the representative $\varphi^{\prime}$.

We now consider the case when $m=0$ and $n, r$ are arbitrary. In this case we may mix both definitions. More precisely we have the following statement, which follows from Proposition 5.8.5:

11.2.1. Proposition-Definition. - Let $\varphi$ be a Function in $C_{+}(S[0, n, r])$. Consider the following commutative diagram of projections

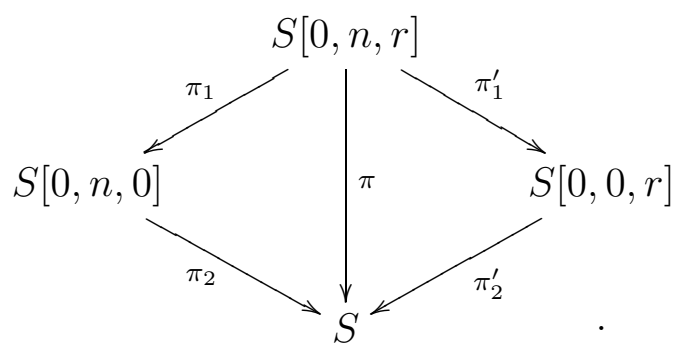

Then $\varphi$ is $\pi_{1}$-integrable if and only if $\pi_{1 !}^{\prime}(\varphi)$ is $\pi_{2}^{\prime}$-integrable. We then say $\varphi$ is $\pi$ integrable. If these conditions hold then $\pi_{2 !} \pi_{1 !}(\varphi)$ and $\pi_{2 !}^{\prime} \pi_{1 !}^{\prime}(\varphi)$ are equal so we may define $\pi_{!}(\varphi)$ to be their commun value.

The case of the projection $\pi: S[1,0,0] \rightarrow S$ has been considered in 9.2 , where we defined the notion of $S$-integrability for $\varphi$ in $C_{+}(S[1,0,0])$ and also the value of $\pi_{!}(\varphi)$ when $\varphi$ is $S$-integrable. We can go one step further thanks to the following: 
11.2.2. Proposition-Definition. - Let $\varphi$ be a Function in $C_{+}(S[1, n, r])$. Consider the following commutative diagram of projections

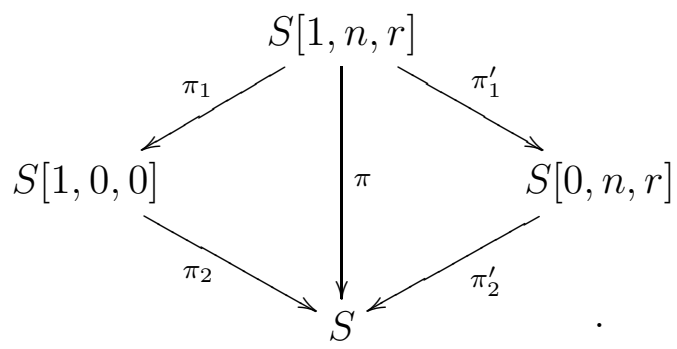

Then the following conditions are equivalent:

(1) $\varphi$ is $\pi_{1}$-integrable and $\pi_{1 !}(\varphi)$ is $\pi_{2}$-integrable.

(2) $\varphi$ is $\pi_{1}^{\prime}$-integrable and $\pi_{1 !}^{\prime}(\varphi)$ is $\pi_{2}^{\prime}$-integrable.

Furthermore, if these conditions are satisfied, then $\pi_{2 !} \pi_{1 !}(\varphi)=\pi_{2 !}^{\prime} \pi_{1 !}^{\prime}(\varphi)$. We shall say $\varphi$ is $S$-integrable if it satisfies conditions (1) and (2) and we shall then define $\pi_{!}(\varphi)$ to be the commun value of $\pi_{2 !} \pi_{1 !}(\varphi)$ and $\pi_{2 !}^{\prime} \pi_{1 !}^{\prime}(\varphi)$.

Proof. - Let $\varphi$ be a Function in $C_{+}(S[1, n, r])$. Choose a cell decomposition of $S[1,0,0]$ which is adapted to $\pi_{1 !}(\varphi)$. For every cell $Z_{1} \subset S[1,0,0]$ with presentation $\tilde{\lambda}: Z_{1} \rightarrow Z_{1}^{\prime} \subset S\left[1, n^{\prime}, r^{\prime}\right]$ in this decomposition let $Z$ be its inverse image of $Z_{1}$ under $\pi_{1}$.

11.2.3. Claim. - The cells $Z$ obtained that way form a cell decomposition of $S[1, n, r]$, adapted to $\varphi$, and having as presentation

$$
\tilde{\lambda} \circ \pi_{1} \times \operatorname{id}_{h[0, n, r]}:\left\{\begin{array}{l}
Z \rightarrow Z^{\prime} \subset S\left[1, n+n^{\prime}, r+r^{\prime}\right] \\
(x, y) \mapsto(\tilde{\lambda}(x), y) .
\end{array}\right.
$$

Proof. - The claim can be easily verified when $n=0$ and when $r=0$ and follows in general by factorizing $\pi_{1}$ into projections $S[1, n, r] \rightarrow S[1,0, r] \rightarrow S[1,0,0]$.

We now consider the following commutative diagram, with $\lambda: Z \rightarrow Z^{\prime}$ and $\tilde{\lambda}: Z_{1} \rightarrow Z_{1}^{\prime}$ as above and where we use the corresponding projections:

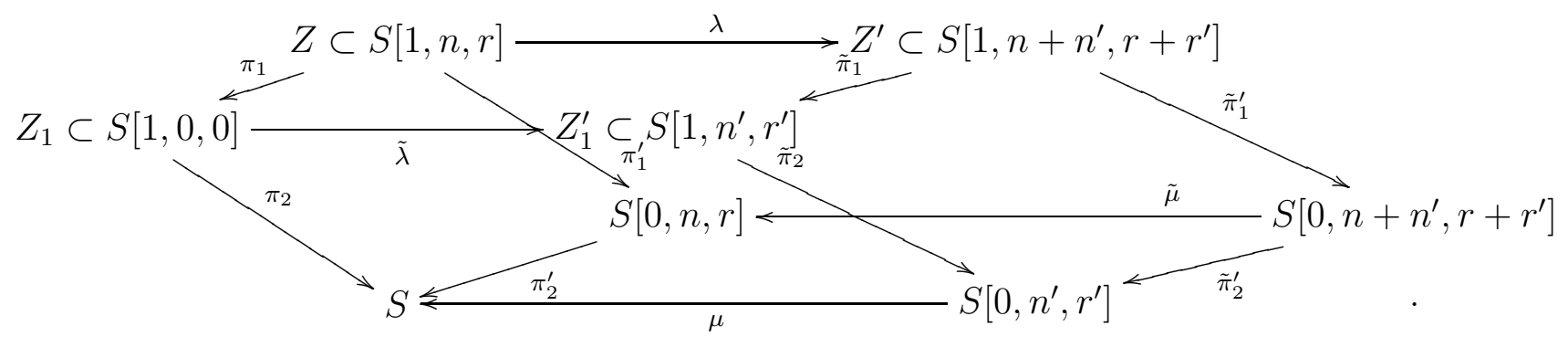

There is a unique $\psi^{\prime}$ such that $\psi=\lambda^{*}\left(\psi^{\prime}\right)$. 
(a) It follows from the claim and the construction in 9.2 that the statement we want to prove is verified for $\left[\psi^{\prime}\right]$, that is, $\left[\psi^{\prime}\right]$ is $\tilde{\pi}_{1}$-integrable and $\tilde{\pi}_{1 !}\left(\left[\psi^{\prime}\right]\right)$ is $\tilde{\pi}_{2^{-}}$ integrable if and only if $\left[\psi^{\prime}\right]$ is $\tilde{\pi}_{1}^{\prime}$-integrable and $\tilde{\pi}_{1 !}^{\prime}\left(\left[\psi^{\prime}\right]\right)$ is $\tilde{\pi}_{2}^{\prime}$-integrable; if these conditions are satisfied, then $\tilde{\pi}_{2 !} \tilde{\pi}_{1 !}\left(\left[\psi^{\prime}\right]\right)=\tilde{\pi}_{2 !}^{\prime} \tilde{\pi}_{1 !}^{\prime}\left(\left[\psi^{\prime}\right]\right)$.

(b) Let us remark that $[\psi]$ is $\pi_{1}$-integrable if and only is $\left[\psi^{\prime}\right]$ is $\tilde{\pi}_{1}$-integrable and $\tilde{\pi}_{1 !}\left(\left[\psi^{\prime}\right]\right)$ is $\tilde{\lambda}^{-1}$-integrable, and that in this case $\left(\tilde{\lambda}^{-1}\right) ! \tilde{\pi}_{1 !}\left(\left[\psi^{\prime}\right]\right)=\pi_{1 !}([\psi])$. This follows from Proposition 5.8.6 and the functoriality of the so far constructed direct images for projections. By the claim and the construction of integration in relative dimension 1 in $9.2, \pi_{1 !}([\psi])$ is $\pi_{2}$-integrable if and only if $\tilde{\pi}_{1 !}\left(\left[\psi^{\prime}\right]\right)$ is $\tilde{\pi}_{2}$-integrable and $\tilde{\pi}_{2 !} \tilde{\pi}_{1 !}\left(\left[\psi^{\prime}\right]\right)$ is $\mu$-integrable. If all the previous conditions are satisfied, then $\pi_{2 !} \pi_{1 !}([\psi])=\mu ! \tilde{\pi}_{2 !} \tilde{\pi}_{1 !}\left(\left[\psi^{\prime}\right]\right)$.

(c) By construction of integration in relative dimension 1, $[\psi]$ is $\pi_{1}^{\prime}$-integrable if and only if $\left[\psi^{\prime}\right]$ is $\tilde{\pi}_{1}^{\prime}$-integrable and $\tilde{\pi}_{1 !}^{\prime}\left(\left[\psi^{\prime}\right]\right)$ is $\tilde{\mu}$-integrable. If this holds, then $\pi_{1 !}^{\prime}([\psi])=\tilde{\mu}_{!} \tilde{\pi}_{1 !}^{\prime}\left(\left[\psi^{\prime}\right]\right)$. Furthermore it follows from Proposition-Definition[11.2.1 that for a Function $g$ in $C_{+}\left(S\left[0, n+n^{\prime}, r+r^{\prime}\right]\right)$ the condition $g$ is $\tilde{\mu}$-integrable and $\tilde{\mu}_{!}(g)$ is $\pi_{2}^{\prime}$-integrable is equivalent to $g$ is $\tilde{\pi}_{2}^{\prime}$-integrable and $\tilde{\pi}_{2 !}^{\prime}(g)$ is $\mu$-integrable and implies that $\pi_{2 !}^{\prime} \tilde{\mu}_{!}(g)=\mu_{!} \tilde{\pi}_{2 !}^{\prime}(g)$.

The statement we have to prove follows directly from the conjunction of (a), (b), and $(c)$.

Now we would like to define $\mathrm{I}_{S} C_{+}(S[n, m, r])$ by induction on $n$ by using a factorization

$$
S[m, n, r] \stackrel{q}{\longrightarrow} S[m-1, n, r] \stackrel{p}{\longrightarrow} S,
$$

with $p$ and $q$ projections, by saying $\varphi$ in $C_{+}(S[m, n, r])$ will be $S$-integrable if it is $S[m-1, n, r]$-integrable and $q_{!}(\varphi)$ is $S$-integrable and setting $\pi_{!}(\varphi):=p_{!}\left(q_{!}(\varphi)\right)$.

Since there are $m$ different projections $S[m, n, r] \rightarrow S[m-1, n, r]$, the factorization (11.2.1) is not unique, and we have to check this definition is independent of the factorization.

By induction it is enough to consider the case $(m, n, r)=(2,0,0)$. Using a bicell decomposition thanks to Proposition 7.4.1 it is enough to prove the following:

11.2.4. Proposition. - Let $Z$ be a bicell in $S[2,0,0]$. Denote by $p_{1}$ and $p_{2}$ the two projections $S[2,0,0] \rightarrow S[1,0,0]$. Then $\left[\mathbf{1}_{Z}\right]$ is $p_{1}$-integrable and $p_{1 !}\left(\left[\mathbf{1}_{Z}\right]\right)$ is $S$ integrable if and only if $\left[\mathbf{1}_{Z}\right]$ is $p_{2}$-integrable and $p_{2 !}\left(\left[\mathbf{1}_{Z}\right]\right)$ is $S$-integrable. If these conditions hold, then $p_{!}\left(p_{1 !}\left(\left[\mathbf{1}_{Z}\right]\right)\right)=p_{!}\left(p_{2 !}\left(\left[\mathbf{1}_{Z}\right]\right)\right)$.

Proof. - Let $Z$ be bicell $Z \subset S[2,0,0]$ with presentation $\lambda: Z \rightarrow Z^{\prime}=Z_{C, \ldots} \subset$ $S[2, n, r]$. Let us first note it is enough to prove the statement of the proposition when $\lambda$ is the identity and that, in this case, the integrability conditions are always 
satisfied. To check that, let us consider the commutative diagram

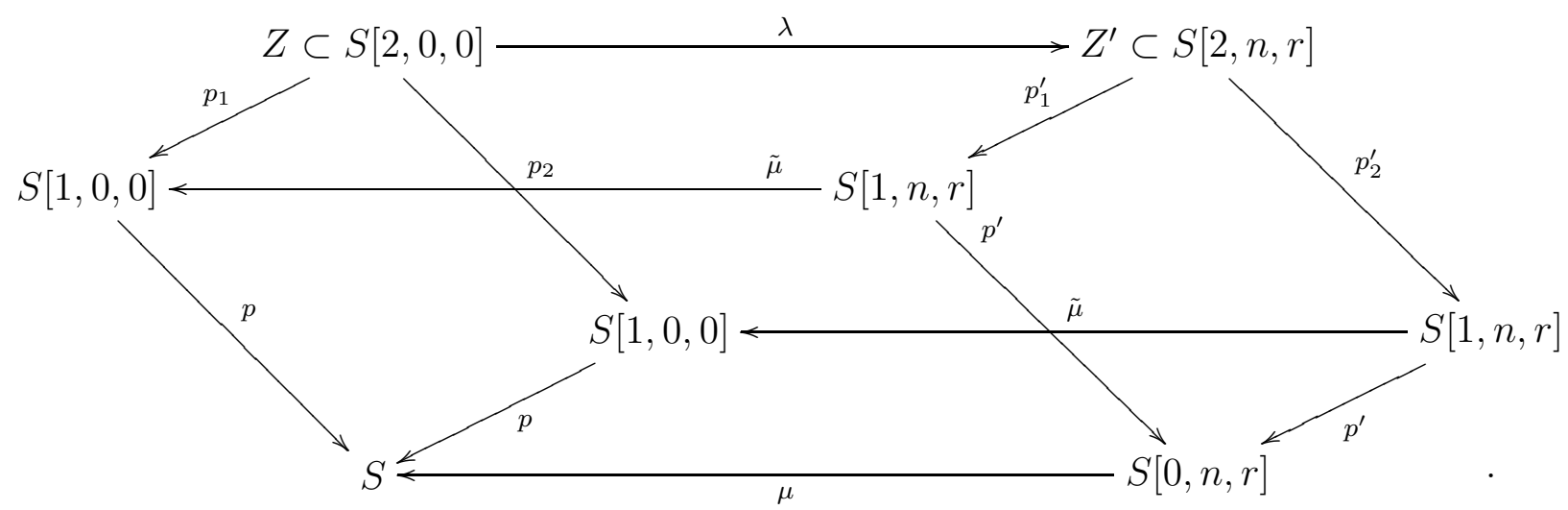

Note first that $\left[\mathbf{1}_{Z}\right]$ is $p_{1}$-integrable if and only if $p_{1 !}^{\prime}\left(\left[\mathbf{1}_{Z^{\prime}}\right]\right)$ is $\tilde{\mu}$-integrable and then $p_{1 !}\left(\left[\mathbf{1}_{Z}\right]\right)=\tilde{\mu}_{!} p_{1 !}^{\prime}\left(\left[\mathbf{1}_{Z^{\prime}}\right]\right)$. Indeed this follows from Proposition-Definition 11.2.2, since $\left[\mathbf{1}_{Z}\right]=\left(\lambda^{-1}\right)_{!}\left(\left[\mathbf{1}_{Z^{\prime}}\right]\right)$. Hence by Proposition-Definition [11.2.2 again, the condition $\left[\mathbf{1}_{Z}\right]$ is $p_{1}$-integrable and $p_{1 !}\left(\left[\mathbf{1}_{Z}\right]\right)$ is $p$-integrable is equivalent to $p_{! !}^{\prime} p_{1 !}^{\prime}\left(\left[\mathbf{1}_{Z^{\prime}}\right]\right)$ is $\mu$ integrable and then $p_{!} p_{1 !}\left(\left[\mathbf{1}_{Z}\right]\right)=\mu_{!} p_{!}^{\prime} p_{1 !}^{\prime}\left(\left[\mathbf{1}_{Z^{\prime}}\right]\right)$. Since we know that $p_{!}^{\prime} p_{1 !}^{\prime}\left(\left[\mathbf{1}_{Z^{\prime}}\right]\right)=$ $p_{!}^{\prime} p_{2 !}^{\prime}\left(\left[\mathbf{1}_{Z^{\prime}}\right]\right)$, we can go the other way back, replacing $p_{1}$ and $p_{1}^{\prime}$ by $p_{2}$ and $p_{2}^{\prime}$, in order to get the required result. Hence, we may now assume that $\lambda$ is the identity.

We consider first the case where $Z$ is $(1,1)$-bicell. As we just explained, we may assume $Z=Z_{C, \alpha, \beta, \xi, \eta, c, d}$, the definable subassignment of $S \times h_{\mathbb{A}_{k(t))}^{1}} \times h_{\mathbb{A}_{k(t))}^{1}}$ defined by

$$
\begin{gathered}
y \in C \\
\operatorname{ord}(z-d(y, u))=\alpha(y) \\
\overline{\operatorname{ac}}(z-d(y, u))=\xi(y) \\
\operatorname{ord}(u-c(y))=\beta(y) \\
\overline{\operatorname{ac}}(u-c(y))=\eta(y),
\end{gathered}
$$

where $y$ denotes the $S$-variable, $z$ the first $\mathbb{A}_{k(t))}^{1}$-variable and $u$ the second $\mathbb{A}_{k(t))^{-}}^{1}$ variable. Furthermore, either $d(y, u)$ is a function of $y$ or $d(y, u)$ is injective as a function of $u$ for every $y$ in $C$. First let us note that $\left[\mathbf{1}_{Z}\right]$ is $p_{2}$-integrable and that $p_{2 !}\left(\left[\mathbf{1}_{Z}\right]\right)=\left[\mathbf{1}_{Z_{2}}\right] \mathbb{L}^{-\alpha-1}$ with $Z_{2}$ the 1-cell

$$
\begin{gathered}
y \in C \\
\operatorname{ord}(u-c(y))=\beta(y) \\
\overline{\operatorname{ac}}(u-c(y))=\eta(y) .
\end{gathered}
$$

It follows that $p_{2 !}\left(\left[\mathbf{1}_{Z}\right]\right)$ is $p$-integrable and $p_{!}\left(p_{2 !}\left(\left[\mathbf{1}_{Z}\right]\right)\right)=\mathbb{L}^{-\alpha-1} \mathbb{L}^{-\beta-1}\left[\mathbf{1}_{C}\right]$.

If $d(y, u)$ is constant as a function of $u$, our $(1,1)$-cell is a product of 1-cells and the result is clear. Let us assume $d(y, u)$ is injective as a function of $u$. After refining the cell decomposition, which is allowed, we may assume the order of the jacobian 
of $d(y, u)$, viewed as a function of the variable $u$ only, is the form $\gamma(y)$, with $\gamma$ a function of $y$ only (and not of $u$ ).

To compute $p_{!}\left(p_{1 !}\left(\left[\mathbf{1}_{Z}\right]\right)\right)$, we shall first prove the following special case.

11.2.5. Lemma. - With the previous notations, consider the definable subassignment $Z$ of $S \times h_{\mathbb{A}_{k(t))}^{1}} \times h_{\mathbb{A}_{k((t))}^{1}}$ defined by

$$
\begin{gathered}
y \in C \\
\operatorname{ord}(z-u)=\alpha(y) \\
\overline{\operatorname{ac}}(z-u)=\xi(y) \\
\operatorname{ord}(u-c(y))=\beta(y) \\
\overline{\operatorname{ac}}(u-c(y))=\eta(y) .
\end{gathered}
$$

Then $\left[\mathbf{1}_{Z}\right]$ is integrable rel. $p_{1}$ and $p_{2}, p_{1 !}\left(\left[\mathbf{1}_{Z}\right]\right)$ and $p_{2 !}\left(\left[\mathbf{1}_{Z}\right]\right)$ are S-integrable and $p_{!}\left(p_{1 !}\left(\left[\mathbf{1}_{Z}\right]\right)\right)=p_{!}\left(p_{2 !}\left(\left[\mathbf{1}_{Z}\right]\right)\right)$.

Proof. - By partitioning $C$ we may assume we are in one of the following 4 cases.

If $\beta>\alpha$ on $C$, then $Z$ may be rewritten as

$$
\begin{gathered}
y \in C \\
\operatorname{ord}(z-c(y))=\alpha(y) \\
\overline{\operatorname{ac}}(z-c(y))=\xi(y) \\
\operatorname{ord}(u-c(y))=\beta(y) \\
\overline{\operatorname{ac}}(u-c(y))=\eta(y),
\end{gathered}
$$

which is a product of 1-cells and the result is clear.

Similarly, if $\beta<\alpha$, resp. $\beta=\alpha$ and $\xi+\eta \neq 0, Z$ may be rewritten as

$$
\begin{gathered}
y \in C \\
\operatorname{ord}(z-u)=\alpha(y) \\
\overline{\operatorname{ac}}(z-u)=\xi(y) \\
\operatorname{ord}(z-c(y))=\beta(y) \\
\overline{\operatorname{ac}}(z-c(y))=\eta(y),
\end{gathered}
$$

and

$$
\begin{gathered}
y \in C \\
\operatorname{ord}(z-c(y))=\alpha(y) \\
\overline{\operatorname{ac}}(z-c(y))=\xi(y)+\eta(y) \\
\operatorname{ord}(u-c(y))=\alpha(y) \\
\overline{\operatorname{ac}}(u-c(y))=\eta(y),
\end{gathered}
$$

respectively, in which case the result is clear by symmetry. 
Finally, if $\beta=\alpha$ and $\xi+\eta=0, Z$ may be rewritten as

$$
\begin{gathered}
y \in C \\
\operatorname{ord}(z-c(y))>\alpha(y) \\
\operatorname{ord}(u-c(y))=\alpha(y) \\
\overline{\operatorname{ac}}(u-c(y))=\eta(y),
\end{gathered}
$$

in which case the result is also quite clear.

Now, we want to compute $p_{1 !}\left(\left[\mathbf{1}_{Z}\right]\right)$. Let consider the image $W$ of

$$
\begin{gathered}
y \in C \\
\operatorname{ord}(u-c(y))=\beta(y) \\
\overline{\operatorname{ac}}(u-c(y))=\eta(y)
\end{gathered}
$$

by $(y, u) \mapsto\left(y, u^{\prime}=d(y, u)\right)$. We denote by $Z^{\prime}$ the subassignement

$$
\begin{gathered}
\operatorname{ord}\left(z-u^{\prime}\right)=\alpha(y) \\
\overline{\operatorname{ac}}\left(z-u^{\prime}\right)=\xi(y) \\
\left(y, u^{\prime}\right) \in W .
\end{gathered}
$$

By Proposition 9.3 .2 (change of variable formula in relative dimension 1 ), $p_{1 !}$ ([1 $\left.\mathbf{1}_{Z}\right]$ ) is equal to $p_{1 !}\left(\left[\mathbf{1}_{Z^{\prime}}\right]\right) \mathbb{L}^{\gamma}$. On the other hand, after applying cell decomposition to $W$, which as we already remarked is allowed here, we deduce from Lemma 11.2.5 that $\left[\mathbf{1}_{Z^{\prime}}\right]$ is integrable rel. $p_{1}$ and $p_{2}, p_{1 !}\left(\left[\mathbf{1}_{Z^{\prime}}\right]\right)$ and $p_{2 !}\left(\left[\mathbf{1}_{Z^{\prime}}\right]\right)$ are $S$-integrable and $p_{!}\left(p_{1 !}\left(\left[\mathbf{1}_{Z^{\prime}}\right]\right)\right)=p_{!}\left(p_{2 !}\left(\left[\mathbf{1}_{Z^{\prime}}\right]\right)\right)$. But $p_{!}\left(p_{2 !}\left(\left[\mathbf{1}_{Z^{\prime}}\right]\right)\right)$ is quite easy to compute, being nothing else than $\mathbb{L}^{-\alpha-1} \mu_{S}(W)$. Hence we get that

$$
p_{1 !}\left(\left[\mathbf{1}_{Z}\right]\right)=\mathbb{L}^{-\alpha-1} \mu_{S}(W) \mathbb{L}^{\gamma} .
$$

Since $\mu_{S}(W)=\mathbb{L}^{-\gamma} \mathbb{L}^{-\beta-1}\left[\mathbf{1}_{C}\right]$, by Proposition 9.3 .2 again, it follows finally that

$$
p_{1 !}\left(\left[\mathbf{1}_{Z}\right]\right)=\mathbb{L}^{-\alpha-1} \mathbb{L}^{-\beta-1}\left[\mathbf{1}_{C}\right],
$$

as required.

We consider now the case of a bicell of type $(1,0)$. As above, we may assume $Z=Z_{C, \beta, \gamma, \eta, c, d}^{\prime}$ is

$$
\begin{gathered}
y \in C \\
z=d(y, u) \\
\operatorname{ord}(u-c(y))=\beta(y) \\
\overline{\operatorname{ac}}(u-c(y))=\eta(y) .
\end{gathered}
$$

Furthermore, either $d(y, u)$ is a function of $y$ or $d(y, u)$ is injective as a function of $u$ for every $y$ in $C$. If $d(y, u)$ is constant as a function of $u$, our $(1,0)$-cell is a product of a 0 -cell and a 1-cell and the result is clear. Let us assume $d(y, u)$ is injective as a function of $u$. 
As we already remarked, we may refine the cell decomposition in order to assume the order of the jacobian of $d(y, u)$, viewed as a function of the variable $u$ only, is a the form $\gamma(y)$, with $\gamma$ a function of $y$ only.

The projection $p_{1}$ induces a definable isomorphism $\lambda_{1}: Z \rightarrow Z_{1}$ between $Z$ and its image $Z_{1}$. By definition, $p_{1 !}\left(\left[\mathbf{1}_{Z}\right]\right)=\mathbb{L}^{\text {ordjac } \lambda_{1} \circ \lambda_{1}^{-1}}\left[\mathbf{1}_{Z_{1}}\right]$. Similarly, $p_{2}$ induces a definable isomorphism $\lambda_{2}: Z \rightarrow Z_{2}$, with $Z_{2}$ defined by

$$
\begin{gathered}
y \in C \\
\operatorname{ord}(u-c(y))=\beta(y) \\
\overline{\operatorname{ac}}(u-c(y))=\eta(y)
\end{gathered}
$$

and $p_{2 !}\left(\left[\mathbf{1}_{Z}\right]\right)=\mathbb{L}^{\text {ordjac } \lambda_{2} \circ \lambda_{2}^{-1}}\left[\mathbf{1}_{Z_{2}}\right]$. Write $\pi_{i}: Z_{i} \rightarrow C$ for the restrictions of $p$ to $Z_{i}$ for $i=1,2$.

Set $\lambda: \lambda_{1} \circ \lambda_{2}^{-1}: Z_{2} \rightarrow Z_{1}$. It is induced by $(y, u) \mapsto(y, d(y, u))$, hence, ordjac $\lambda=$ $\gamma(y)$ depends only on $y$. After refining the cell decomposition, we may assume that ordjac $\lambda_{2} \circ \lambda_{2}^{-1}$ also depends only on $y$, that is, there exists some function $\mu_{2}$ on $C$ such that $\pi_{2}^{*} \mu_{2}=\operatorname{ordjac} \lambda_{2} \circ \lambda_{2}^{-1}$ (almost everywhere). Refining again the cell decomposition, we may assume that there also exists a function $\mu_{1}$ on $C$ such that $\pi_{1}^{*} \mu_{1}=\operatorname{ordjac} \lambda_{1} \circ \lambda_{1}^{-1}$ (almost everywhere). By the chain rule (Proposition 8.4.1) applied to $\lambda$, we find

$$
\pi_{2}^{*}(\gamma)=\pi_{2}^{*}\left(\mu_{1}\right)-\pi_{2}^{*}\left(\mu_{2}\right)
$$

from which the relation

$$
\mu_{1}=\gamma+\mu_{2}
$$

follows. By the projection formula, which is valid in this case by construction, we have

$$
p_{!} p_{1 !}\left(\left[\mathbf{1}_{Z}\right]\right)=\mathbb{L}^{\mu_{1}} p_{!}\left(\left[\mathbf{1}_{Z_{1}}\right]\right)
$$

and

$$
p_{!} p_{2 !}\left(\left[\mathbf{1}_{Z}\right]\right)=\mathbb{L}^{\mu_{2}} p_{!}\left(\left[\mathbf{1}_{Z_{2}}\right]\right)
$$

Since

$$
p_{!}\left(\left[\mathbf{1}_{Z_{2}}\right]\right)=\mathbb{L}^{\gamma} p_{!}\left(\left[\mathbf{1}_{Z_{1}}\right]\right)
$$

by Proposition 9.3.2, we deduce the required result by Equation (11.2.2).

We are now left with the last two cases which are much easier. As above, we may assume $Z=Z_{C, \alpha, \xi, c, d}^{\prime \prime}$ or $Z=Z_{C, c, d}^{\prime \prime \prime}$. In both cases the result is clear since $Z$ is a product of cells.

Let us define $\mathrm{I}_{S} C_{+}(S[n, m, r])$ by induction on $n$ by using a factorization

$$
S[m, n, r] \stackrel{q}{\longrightarrow} S[m-1, n, r] \stackrel{p}{\longrightarrow} S,
$$

with $p$ and $q$ projections, and saying $\varphi$ in $C_{+}(S[m, n, r])$ is $S$-integrable if it is $S[m-$ $1, n, r]$-integrable and $q_{!}(\varphi)$ is $S$-integrable and setting $\pi_{!}(\varphi):=p_{!}\left(q_{!}(\varphi)\right)$. It follows from Proposition 11.2.4 that these definitions are independent under permutation of the coordinates on $\mathbb{A}_{k(t))}^{m}$. 
11.3. - We now define $\mathrm{I}_{S} C_{+}(S \times Y)$ and $\pi_{\text {! }}$, with $\pi$ the projection $S \times Y \rightarrow S$, when $Y$ is a definable subassignment of $h[m, n, r]$. This is done as follows. We denote by $i: S \times Y \rightarrow S[m, n, r]$ the inclusion and by $\tilde{\pi}$ the projection $S[m, n, r] \rightarrow S$. To any Function $\varphi$ in $C_{+}^{d}(S \times Y)$, we assign the Function $\tilde{\varphi}:=i_{!}(\varphi)$ in $C_{+}^{d}(S[m, n, r])$, which is the "(class of the) Function $\varphi$ extended by zero outside $S \times Y$ ". We shall say $\varphi$ is $S$-integrable if $\tilde{\varphi}$ is $S$-integrable and we shall set $\pi_{!}(\varphi):=\tilde{\pi}_{!}(\tilde{\varphi})$.

11.4. - Before going further in the construction of $\pi_{!}$, we shall state some useful properties that follow from what we already did in 11.2 .

We already have the following form of A0 and A1 for projections:

11.4.1. Proposition. - Consider a diagram of projections

$$
\pi: S \times Y \times Z \stackrel{q}{\longrightarrow} S \times Y \stackrel{p}{\longrightarrow} S .
$$

A Function $\varphi$ in $C_{+}(S \times Y \times Z)$ is $S$-integrable if and only if it is $S \times Y$-integrable and $q_{!}(\varphi)$ is $S$-integrable. If this holds, then

$$
\pi_{!}(\varphi)=p_{!} q_{!}(\varphi)
$$

Proof. - One may assume $Y$ and $Z$ are of the form $h[m, n, r]$ and $h\left[m^{\prime}, n^{\prime}, r^{\prime}\right]$, respectively. The result then follows by induction from Proposition-Definition 11.2.1 and Proposition-Definition 11.2.2.

Also the projection formula A3 holds for projections:

11.4.2. Proposition. - Let $S$ and $Y$ be in $\operatorname{Def}_{k}$ and let $\pi: S \times Y \rightarrow S$ denote the projection. For every $\alpha$ in $\mathscr{C}_{+}(S)$, and every $\beta$ in $\mathrm{I}_{S} C_{+}(S \times Y), \pi^{*}(\alpha) \beta$ belongs to $\mathrm{I}_{S} C_{+}(S \times Y)$ and $\pi_{!}\left(\pi^{*}(\alpha) \beta\right)=\alpha \pi_{!}(\beta)$.

Proof. - One may assume $Y=h[m, n, r]$. If $m=0$, the statement follows from the fact that $\mathrm{I}_{S} \mathscr{C}_{+}(S[0, n, r])$ is a $\mathscr{C}_{+}(S)$-module and that $\pi_{!}$is $\mathscr{C}_{+}(S)$-linear. The case $m=1$ follows from the case $m=0$ by construction, and the general case is deduced by induction on $m$.

We also have the following special case of Theorem 12.1.1;

11.4.3. Proposition. - Let $S$ and $Y$ be in $\operatorname{Def}_{k}$. Let $Z$ be a definable subassignment of $S \times Y$. Assume the projection $\pi: S \times Y \rightarrow S$ induces an isomorphism $\lambda$ between $Z$ and $S$. Then $\left[\mathbf{1}_{Z}\right]$, viewed as a Function in $C_{+}(S \times Y)$, is $S$-integrable and

$$
\pi_{!}\left(\left[\mathbf{1}_{Z}\right]\right)=\mathbb{L}^{\text {ordjac } \lambda \circ \lambda^{-1}}\left[\mathbf{1}_{S}\right]
$$

Proof. - We may assume $Y=h[m, n, r]$, so that $S \times Y=S[m, n, r]$. For $m=0$, the result is clear, for $m=1$ it follows from construction. The general case is proved by induction on $m$ using the chain rule Proposition 8.4.1. 
11.5. Definable injections. - Let $i: X \rightarrow Y$ be a morphism in $\operatorname{Def}_{k}$. We shall assume $i$ is injective, which means that $i$ induces a definable isomorphism between $X$ and $i(X)$.

For every Function $\varphi$ in $C_{+}(X)$, we define a Function $i_{+}(\varphi)$ in $C_{+}(Y)$ as follows. We shall define $i_{+}$on $C_{+}^{d}(X)$, and then extend to the whole $C_{+}(X)$ by linearity. Take $\varphi=[\psi]$ in $C_{+}^{d}(X)$. We can choose a definable subassignment $Z$ of $X$ of dimension $d$ such that $\psi$ is zero outside $Z$. The morphism $i$ induces a definable isomorphism $\gamma_{Z}$ between $Z$ and $i(Z)$. Consider the Function $\tilde{\psi}:=\mathbb{L}^{\operatorname{ordjac}} \gamma_{Z} \circ \gamma_{Z}^{-1}\left(\gamma_{Z}^{-1}\right)^{*}(\psi)$ on $i(Z)$. We define the Function $\tilde{\varphi}$ on $Y$ to be 0 outside $i(Z)$ and to be equal to $\tilde{\psi}$ on $i(Z)$, which is independent of the choice of $Z$.

In fact we shall see later (cf. Proposition 12.1.2) that $i_{+}$is nothing else than $i_{!}$.

This gives support to the following:

11.5.1. Proposition. - Let $i: X \rightarrow Y$ and $j: Y \rightarrow Z$ be morphisms in Def $_{k}$. Assume the morphims $i$ and $j$ are injective. Then $(j \circ i)_{+}=j_{+} \circ i_{+}$.

Proof. - Follows directly from Proposition 8.4.1.

We shall need later the following:

11.5.2. Lemma. - Let $i: Y \rightarrow W$ be an injective morphism in $\operatorname{Def}_{k}$, and consider the commutative diagram

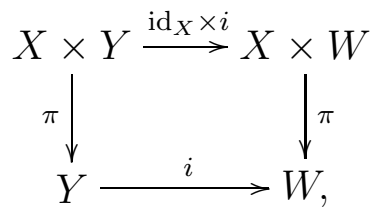

where $\pi$ denotes the projections. A Function $\varphi$ in $C_{+}(X \times Y)$ is $Y$-integrable if and only if $\left(\operatorname{id}_{X} \times i\right)_{+}(\varphi)$ is $\pi$-integrable. When these conditions hold, we have

$$
\pi_{!}\left(\left(\operatorname{id}_{X} \times i\right)_{+}(\varphi)\right)=i_{+}\left(\pi_{!}(\varphi)\right) .
$$

Proof. - We may assume $X=h[m, n, r]$, so that $X \times Y=Y[m, n, r]$. When $m=0$, the statement follows from Proposition 5.8.6. Let us now consider the case $m=1$, $n=r=0$. Take $\varphi$ in $C_{+}(Y[1,0,0])$ and consider a cell decomposition $\mathcal{Z}$ of adapted to (some representative of) $\varphi$ and to $\operatorname{ordjac}\left(\operatorname{id}_{X} \times i\right)$. Note that the image of $\mathcal{Z}$ in $W[1,0,0]$ is adapted to $\left(\operatorname{id}_{X} \times i\right)_{+}(\varphi)$. The result now follows from the construction of $\pi_{\text {! }}$ made in $\S 9$ since this construction reduces the case $m=1$ to the case $m=0$. The general case follows by induction using Proposition 11.4.1.

11.6. Push-forward for the structural morphism. - Let $f: X \rightarrow S$ be a morphism in $\operatorname{Def}_{S}$.

We consider the following canonical factorization of $f$ :

$$
X \stackrel{i_{f}}{\longrightarrow} X \times S \stackrel{\pi_{f}}{\longrightarrow} S,
$$


where $i_{f}$ is the graph morphism $x \mapsto(x, f(x))$ and $\pi_{f}$ the canonical projection. The graph morphism $i_{f}$ induces a definable isomorphism $\gamma_{f}$ between $X$ and the graph of $f, \Gamma_{f}:=i_{f}(X)$.

We shall say a Function $\varphi$ in $C_{+}(X)$ is $S$-integrable if $i_{f+}(\varphi)$ is $S$-integrable. When this holds we shall set

$$
f_{!}(\varphi):=\pi_{f !}\left(i_{f+}(\varphi)\right) .
$$

One should first check that when $f$ is a projection, one recovers the previous definitions:

11.6.1. Lemma. - If $f: Y \times S \rightarrow S$ is the projection on the second factor, then the above definitions coincide with the ones in 11.3.

Proof. - Let us first consider the case when $f$ is the identity $\mathrm{id}_{S}$. It then follows from Proposition 11.4.3 applied to the projection $\Gamma_{\mathrm{id}_{S}} \rightarrow S$, that, for every Function $\varphi$ in $C_{+}(S), i_{\mathrm{id}_{S}+}(\varphi)$ is $S$-integrable and that $\pi_{\mathrm{id}_{S} !} \circ i_{\mathrm{id}_{S}+}$ is the identity. For the general case, let us consider the commutative diagram

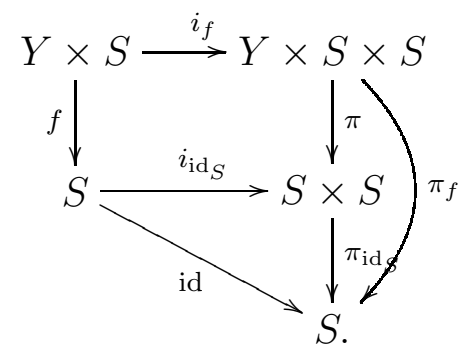

By Lemma 11.5.2, a Function $\varphi$ in $C_{+}(Y \times S)$ is $S$-integrable if and only if $i_{f+}(\varphi)$ is $S \times S$-integrable, and if this is the case, then

$$
\pi_{!} i_{f+}(\varphi)=i_{\mathrm{id}_{S}+} f_{!}(\varphi)
$$

so that

and

$$
\pi_{\mathrm{id}_{S} !} \pi_{!} i_{f+}(\varphi)=\pi_{\mathrm{id}_{S} !} i_{\mathrm{id}_{S}+} f_{!}(\varphi)
$$

$$
\pi_{f !} i_{f+}(\varphi)=f_{!}(\varphi)
$$

11.7. Push-forward: the general case. - We start from a morphism $f: X \rightarrow$ $Y$ in $\operatorname{Def}_{S}$, that is, a commutative diagram

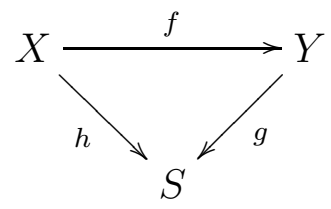

In 11.6 we defined a morphism $f_{!}: \mathrm{I}_{Y} C_{+}(X) \rightarrow \mathrm{I}_{Y} C_{+}(Y)$. By the following Proposition 11.7.1, $f_{\text {! }}$ restricts to a morphism $\mathrm{I}_{S} C_{+}(X) \rightarrow \mathrm{I}_{S} C_{+}(Y)$ that we shall still denote by $f_{!}$. 
11.7.1. Proposition. - A Function $\varphi$ in $C_{+}(X)$ is $S$-integrable if and only if it is $Y$-integrable and $f_{!}(\varphi)$ is $S$-integrable. If these conditions hold then $h_{!}(\varphi)=g_{!} f_{!}(\varphi)$.

Proof. - We have the following commutative diagram:

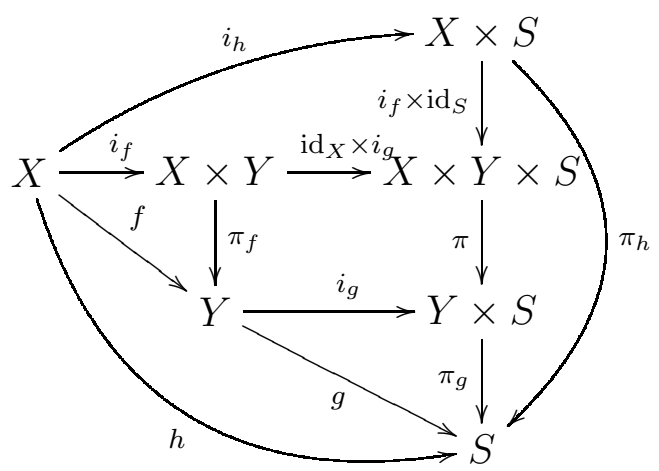

with $\pi$ the projection on the last two factors.

Let $\varphi$ be a Function in $C_{+}(X)$. The following conditions are equivalent: $\varphi$ is $f$-integrable and $f_{!}(\varphi)$ is $g$-integrable $i_{f+}(\varphi)$ is $\pi_{f}$-integrable and $i_{g+} \pi_{f !} i_{f+}(\varphi)$ is $\pi_{g}$-integrable (by Definition)

$\left(\operatorname{id}_{X} \times i_{g}\right)_{+} i_{f+}(\varphi)$ is $\pi$-integrable and $\pi_{!}\left(\operatorname{id}_{X} \times i_{g}\right)_{+} i_{f+}(\varphi)$ is $\pi_{g}$-integrable (by Lemma 11.5.2) $\left(i_{f} \times \operatorname{id}_{S}\right)_{+} i_{h+}(\varphi)$ is $\pi$-integrable and $\pi_{!}\left(i_{f} \times \operatorname{id}_{S}\right)_{+} i_{h+}(\varphi)$ is $\pi_{g}$-integrable (by Lemma 11.5.1) $\left(i_{f} \times \operatorname{id}_{S}\right)_{+} i_{h+}(\varphi)$ is $S$-integrable (by Proposition 11.4.1) $i_{h+}(\varphi)$ is $\pi_{h}$-integrable

(by Lemma 11.7.2) $\varphi$ is $h$-integrable (by Definition).

This proves the first statement in the proposition.

Assume now the previous conditions hold. We have

$$
\begin{aligned}
g_{!} f_{!}(\varphi) & =\pi_{g !} i_{g+} \pi_{f !} i_{f+}(\varphi) & & \text { (by Definition) } \\
& =\pi_{g !} \pi_{!}\left(\operatorname{id}_{X} \times i_{g}\right)_{+} i_{f+}(\varphi) & & \text { (by Lemma 11.5.2) } \\
& =\left(\pi_{g} \circ \pi\right)_{!}\left(\mathrm{id}_{X} \times i_{g}\right)_{+} i_{f+}(\varphi) & & \text { (by Proposition 11.4.1) } \\
& =\left(\pi_{g} \circ \pi\right)_{!}\left(i_{f} \times \mathrm{id}_{S}\right)_{+} i_{h+}(\varphi) & & \text { (by Lemma 11.5.1) } \\
& =\pi_{h !} i_{h+}(\varphi) & & \text { (by Lemma 11.7.2) } \\
& =h_{!}(\varphi) & & \text { (by Definition). }
\end{aligned}
$$


11.7.2. Lemma. - Let $f: X \rightarrow Y$ be a morphism in $\operatorname{Def}_{k}$, let $S$ be in $\operatorname{Def}_{k}$, and consider the commutative diagram

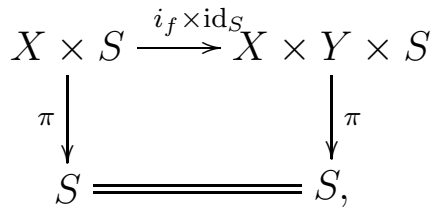

where $\pi$ denotes the projection. A Function $\varphi$ in $C_{+}(X \times S)$ is S-integrable if and only if $\left(i_{f} \times \operatorname{id}_{S}\right)_{+}(\varphi)$ is $S$-integrable. If this holds, then $\pi_{!}(\varphi)=\pi_{!}\left(\left(i_{f} \times \operatorname{id}_{S}\right)_{+}(\varphi)\right)$.

Proof. - We consider the commutative diagram

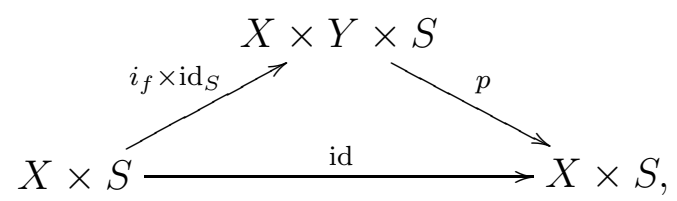

with $p$ the projection. Consider $Z:=\left(i_{f} \times \operatorname{id}_{S}\right)(X \times S)$. The projection $p$ induces an isomorphism $\lambda$ between $Z$ and $X \times S$. It follows from Proposition 11.4.3 that $\left[\mathbf{1}_{Z}\right]$ is $X \times S$-integrable and that $p_{!}\left(\left[\mathbf{1}_{Z}\right]\right)=\mathbb{L}^{\text {ordjac } \lambda \circ \lambda^{-1}}\left[\mathbf{1}_{X \times S}\right]$, hence, by the very definition of $i_{f+}$ and by Propositions 8.4.1 and 11.4.2, we obtain that $p_{!}\left(i_{f} \times \operatorname{id}_{S}\right)_{+}\left(\left[\mathbf{1}_{X \times S}\right]\right)=\left[\mathbf{1}_{X \times S}\right]$. Now, for a general Function $\varphi$ in in $C_{+}(X \times S)$, we get similarly by Proposition 11.4 .2 that $\left(i_{f} \times \mathrm{id}_{S}\right)_{+}(\varphi)$ is $p$-integrable and that $p_{!}\left(i_{f} \times \operatorname{id}_{S}\right)_{+}(\varphi)=\varphi$, after maybe replacing $Z$ by a definable subassignment of smaller dimension. So, $p_{!}\left(i_{f} \times \mathrm{id}_{S}\right)_{+}$is the identity, hence to conclude the proof it is enough to compose with $\pi_{\text {! }}$ and to apply Proposition 11.4.1.

11.8. Conclusion of the proof. - Now we have everything in hand needed to check Axioms A0-9. Axiom A0 follows at once from Proposition 11.7.1, Statements (a) and (b) in A1 are clear by construction. Since A2 and A4 hold by construction for $\pi_{!}$, when $\pi$ is a projection and for $i_{+}$, when $i$ is a definable injection, it follows they hold in general. Similarly, A3 holds for $\pi_{\text {! }}$, when $\pi$ is a projection, by Proposition 11.4.2 and for $i_{+}$, when $i$ is a definable injection, by construction, hence it holds in general. The remaining axioms A5-8 follow from the very constructions and definitions.

\section{Main properties}

Recall in this section, and until section 15, all definable subassignments belong to $\operatorname{Def}_{k}$, so in particular they are affine.

12.1. Change of variable formula. - We can now state the general form of the change of variable formula. 
12.1.1. Theorem. - Let $f: X \rightarrow Y$ be a definable isomorphism between definable subassignments of $K$-dimension $d$. Let $\varphi$ be in $\mathscr{C}_{+}^{\leq d}(Y)$ of $K$-dimension d. Then $\left[f^{*}(\varphi)\right]$ belongs to $\mathrm{I}_{Y} C_{+}^{d}(X)$ and

$$
f_{!}\left(\left[f^{*}(\varphi)\right]\right)=\mathbb{L}^{\operatorname{ordjac} f \circ f^{-1}}[\varphi] .
$$

Proof. - By Proposition 12.1.2, $\left[\mathbf{1}_{X}\right]$ is $Y$-integrable and $f_{!}\left(\left[\mathbf{1}_{X}\right]\right)=\mathbb{L}^{\operatorname{ordjac} f \circ f^{-1}}\left[\mathbf{1}_{Y}\right]$. The result follows, since by $\mathrm{A} 1(\mathrm{~b})$ and the projection formula $\mathrm{A} 3,\left[f^{*}(\varphi)\right]=$ $f^{*}(\varphi)\left[\mathbf{1}_{X}\right]$ is $Y$-integrable and $f_{!}\left(\left[f^{*}(\varphi)\right]\right)=\varphi f_{!}\left(\left[\mathbf{1}_{X}\right]\right)=\mathbb{L}^{\operatorname{ordjac} f \circ f^{-1}}[\varphi]$.

12.1.2. Proposition. - Let $j: X \rightarrow Y$ be a definable injection. Then $\mathrm{I}_{Y} C_{+}(X)=C_{+}(X)$ and $j_{+}=j_{!}$.

Proof. — We factor $j$ as

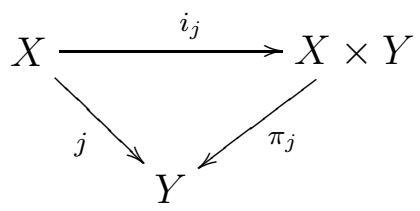

Using compatibility with inclusions A4 and the projection formula A3 it is enough to prove that $i_{j+}\left(\left[\mathbf{1}_{X}\right]\right)$ is $Y$-integrable and that $j_{+}\left(\left[\mathbf{1}_{X}\right]\right)=\pi_{j !} \circ i_{j+}\left(\left[\mathbf{1}_{X}\right]\right)$. This follows from Proposition 11.4.3 and Proposition 8.4.1.

12.2. Integrability of bounded Functions on bounded subassignments. Let $X$ be in $\operatorname{Def}_{k}$. Let $\varphi$ and $\varphi^{\prime}$ be Functions in $C_{+}(X)$. We write $\varphi \leq \varphi^{\prime}$ if there exists a Function $\psi$ in $C_{+}(X)$ such that $\varphi^{\prime}=\varphi+\psi$.

12.2.1. Theorem. - Let $f: X \rightarrow S$ be a morphism in $\operatorname{Def}_{k}$. Let $\varphi$ and $\varphi^{\prime}$ be Functions in $C_{+}(X)$ such that $\varphi \leq \varphi^{\prime}$. If $\varphi^{\prime}$ is $S$-integrable, then $\varphi$ is $S$-integrable.

Proof. - The statement being clear when $f$ is an injection, we assume $f$ is a projection $f: X=S[m, n, r] \rightarrow S$. When $m=0$ the result is quite clear, hence it is enough by induction to consider the case $(m, n, r)=(1,0,0)$ which follows directly from Proposition 9.3.1.

We shall say a subassignment $Z$ of $h[m, n, 0]$ is bounded if there exists a natural number $s$ such that $Z$ is contained in the subassignment $W_{s}$ of $h[m, n, 0]$ defined by ord $x_{i} \geq-s, 1 \leq i \leq m$, where the variables $x_{i}$ run over $h[m, 0,0]$.

12.2.2. Proposition. - If $Z$ is a bounded definable subassignment of $h[m, n, 0]$, then $\left[\mathbf{1}_{Z}\right]$ is integrable. More generally, let $\varphi$ be a Function in $C_{+}(Z)$ of the form $a \otimes \alpha \mathbb{L}^{\beta}\left[\mathbf{1}_{Z}\right]$ with a in $S K_{0}\left(\operatorname{RDef}_{Z}\right)$, $\alpha$ a product of definable morphisms $\alpha_{i}: Z \rightarrow \mathbb{N}$ for $i=1, \ldots, \ell$, and $\beta: Z \rightarrow \mathbb{Z}$ a definable morphism. Assume $Z$ is bounded and the function $\beta$ is bounded above. Then $\varphi$ is integrable.

Proof. - Assume $Z$ is of $K$-dimension $d$. We shall prove the more general statement by induction on the codimension $m-d$, assuming the $\alpha_{i}$ are also bounded above. 
There exists a closed $k((t))$-subvariety $\mathcal{X}$ of dimension $d$ of $\mathbb{A}_{k(t))}^{m}$ such that $Z$ is contained in $\left(h_{\mathcal{X}} \times h_{\mathbb{A}_{k}^{n}}\right) \cap W_{s}$.

When $m=d,\left[\mathbf{1}_{Z}\right] \leq\left[\mathbf{1}_{W_{s}}\right]$. Certainly $\left[\mathbf{1}_{W_{s}}\right]$ is integrable and $\mu\left(\left[\mathbf{1}_{W_{s}}\right]\right)=\mathbb{L}^{-s}$, as can be seen by using a cell decomposition similar to the one in Example 7.2.2. Also any Function of the form $a \otimes \tilde{\alpha} \mathbb{L}^{\tilde{\beta}}\left[\mathbf{1}_{W_{s}}\right]$ with $a$ in $S K_{0}\left(\operatorname{RDef}_{W_{s}}\right), \tilde{\alpha}$ and $\tilde{\beta}$ constant positive numbers, is integrable, hence the statement follows from Theorem 12.2.1 in this case.

Assume now $m>d$. After performing a linear change of coordinates on $\mathbb{A}_{k((t))}^{m}$, which we are allowed to do by Theorem 12.1.1, we may assume that the projection $\mathbb{A}_{k(t))}^{m} \rightarrow \mathbb{A}_{k((t))}^{m-1}$ on the first $m-1$ coordinates restricts to a finite morphism on $\mathcal{X}$.

Denote by $Z^{\prime}$ the image of $Z$ under the projection $p: Z \rightarrow h[m-1, n, 0]$. Note that $Z^{\prime}$ is bounded. Using a cell decomposition adapted to $\varphi$, one may assume that $Z$ is a cell (necessarily a 0-cell) adapted to $\varphi$. By the induction hypothesis it is enough to prove that $\left[\mathbf{1}_{Z}\right]$ is $p$-integrable and that $p_{!}\left[\mathbf{1}_{Z}\right]$ is of the form $a^{\prime} \otimes \alpha^{\prime} \mathbb{L}^{\beta^{\prime}}\left[\mathbf{1}_{Z^{\prime}}\right]$ with $a^{\prime}$ in $S K_{0}\left(\operatorname{RDef}_{Z^{\prime}}\right), \alpha^{\prime}$ a product of definable morphisms $\alpha_{i}^{\prime}: Z^{\prime} \rightarrow \mathbb{N}$, and the $\alpha_{i}^{\prime}$ and $\beta^{\prime}$ bounded above definable functions on $Z^{\prime}$. Let $\lambda: Z \rightarrow Z_{C}$ be a presentation of $Z$ and consider the projections $p^{\prime}: Z_{C} \rightarrow C \subset h\left[m-1, n+n^{\prime}, 0\right], \pi_{1}: Z_{C} \rightarrow Z$, and $\pi_{2}: C \rightarrow Z^{\prime}$. Since the image by $\lambda_{!}$and $\pi_{i}$ ! for $i=1,2$ of Functions of the above form have a similar form, we may in fact assume $Z=Z_{C}, Z^{\prime}=C$ and $p=p^{\prime}$, that is, we may assume $p$ induces an isomorphism between $Z$ and $Z^{\prime}$. Since $Z$ is bounded, it follows from Theorem 7.5.1 that some representative of ordjacp $\circ p^{-1}$ is bounded above on $Z^{\prime}$. Hence, it follows from A8, or from the stronger Theorem 12.1.1, that $p_{!}\left(\left[\mathbf{1}_{Z}\right]\right)=\mathbb{L}^{\beta}\left[\mathbf{1}_{Z^{\prime}}\right]$ with $\beta$ bounded above on $Z^{\prime}$, which finishes the proof in this case.

Let us now consider the case where the functions $\alpha_{i}$ are no more assumed to be bounded. We denote by $\tilde{\alpha}$ the morphism $\left(\alpha_{1}, \ldots, \alpha_{\ell}\right): Z \rightarrow \mathbb{N}^{\ell}$, and, for $n$ in $\mathbb{N}^{\ell}$, we set $Z_{n}:=\tilde{\alpha}^{-1}(n)$ and $\varphi_{n}:=\mathbf{1}_{Z_{n}} \varphi$. By what we already proved, each $\varphi_{n}$ is integrable, and also the Function $\psi:=a \otimes \mathbb{L}^{\beta}\left[\mathbf{1}_{Z}\right]$ is integrable. Since we may factor the projection of $Z \rightarrow h[0,0,0]$ as the composition of $\tilde{\alpha}: Z \rightarrow \mathbb{N}^{\ell}$ with the projection $\mathbb{N}^{\ell} \rightarrow h[0,0,0]$, it follows that the Function $n \mapsto \mu\left(\psi_{n}\right)$ is integrable on $\mathbb{N}^{\ell}$, with $\psi_{n}:=\mathbf{1}_{Z_{n}} \psi$. Hence, by Proposition 4.5.6, we may write $n \mapsto \mu\left(\psi_{n}\right)$ as a finite sum of functions of the form $d \otimes h$ with $d$ in $S K_{0}\left(\operatorname{RDef}_{k}\right)$ and $h$ in $\mathcal{P}(h[0,0, \ell])$ with for each $h \lim _{|n| \rightarrow \infty} \operatorname{deg}_{\mathbb{L}}(h)=-\infty$. But then also $\lim _{|n| \rightarrow \infty} \operatorname{deg}_{\mathbb{L}}(\alpha h)=-\infty$ for each $h$, hence $\lim _{|n| \rightarrow \infty} \operatorname{deg}_{\mathbb{L}}(\varphi)=-\infty$. By Proposition 4.5.6, the function $n \mapsto \mu\left(\varphi_{n}\right)$ is integrable on $\mathbb{N}^{\ell}$, and one deduces that $\varphi$ is integrable.

\section{Integration of general constructible motivic Functions}

13.1. From $C_{+}(X)$ to $C(X)$. - In this section we shall denote by $\iota$ the canonical morphisms $\iota: C_{+}(X) \rightarrow C(X)$ and $\iota: \mathscr{C}_{+}(X) \rightarrow \mathscr{C}(X)$ for $X$ in $\operatorname{Def}_{k}$. 
13.1.1. Proposition. - Fix $S$ in $\operatorname{Def}_{k}$. Let $f: X \rightarrow Y$ be a morphism in $\operatorname{Def}_{S}$. Let $\varphi$ and $\varphi^{\prime}$ be Functions in $C_{+}(X)$ such that $\iota(\varphi)=\iota\left(\varphi^{\prime}\right)$. Assume that $\varphi$ and $\varphi^{\prime}$ are $S$-integrable. Then $\iota\left(f_{!}(\varphi)\right)=\iota\left(f_{!}\left(\varphi^{\prime}\right)\right)$.

Proof. - It is enough to prove the statement for $f$ an injection or a projection. When $f$ is an injection the proof is quite clear. Indeed, if $\iota(\varphi)=\iota\left(\varphi^{\prime}\right)$, we have $\varphi+\psi=\varphi^{\prime}+\psi$ with some $\psi$ in $C_{+}(X)$ which might be not $S$-integrable, but is certainly $f$-integrable (since all Functions in $C_{+}(X)$ are). It follows that $f_{!}(\varphi)+$ $f_{!}(\psi)=f_{!}\left(\varphi^{\prime}\right)+f_{!}(\psi)$, which is enough for our needs. Let us now assume $f$ is a projection. We may assume $f$ is the projection $X=S[m, n, r] \rightarrow Y=S$. The case $(m, n)=(0,0)$ follows directly from Lemma 13.1.2 and the case $(m, r)=(0,0)$ is clear, so we know the statement holds for $X=S[0, n, r]$, and by induction it is enough to prove it also holds for $X=S[1, n, r]$. We explain how an application of cell decomposition reduces to the case $X=S[0, n, r]$. Let $\varphi+\psi=\varphi^{\prime}+\psi$ for some $\psi$. It is enough to consider the case where $\varphi, \varphi^{\prime}$ and $\psi$ all lie in $C_{+}^{d}(X)$. We may assume, using cell decomposition, that $\varphi, \varphi^{\prime}$ and $\psi$ have their support contained in a cell $\lambda: Z \rightarrow Z_{C}$, with $Z$ of $K$-dimension $d$, and that $\varphi=\lambda^{*} p^{*}(h)\left[\mathbf{1}_{Z}\right]$, $\varphi^{\prime}=\lambda^{*} p^{*}\left(h^{\prime}\right)\left[\mathbf{1}_{Z}\right] \psi=\lambda^{*} p^{*}(g)\left[\mathbf{1}_{Z}\right]$, with $h, h^{\prime}$, and $g$ in $\mathscr{C}_{+}(C)$, where $p$ denotes the projection $Z_{C} \rightarrow C \subset S\left[0, n+n^{\prime}, r+r^{\prime}\right]$. Moreover we may assume that $\left[\mathbf{1}_{Z}\right]$ is $C$-integrable. Then $[h]+\left[g^{\prime}\right]=\left[h^{\prime}\right]+\left[g^{\prime}\right]$, and thus $h p_{!}\left(\left[\mathbf{1}_{Z}\right]\right)+g p_{!}\left(\left[\mathbf{1}_{Z}\right]\right)=$ $h^{\prime} p_{!}\left(\left[\mathbf{1}_{Z}\right]\right)+g p_{!}\left(\left[\mathbf{1}_{Z}\right]\right)$. Consider the projection $\pi: C \rightarrow S$. Since $h p_{!}\left(\left[\mathbf{1}_{Z}\right]\right)$ and $h^{\prime} p_{!}\left(\left[\mathbf{1}_{Z}\right]\right)$ are $S$-integrable by construction, it follows from what we already proved that, $\iota\left(\pi_{!}\left(h p_{!}\left(\left[\mathbf{1}_{Z}\right]\right)\right)\right)=\iota\left(\pi_{!}\left(h^{\prime} p_{!}\left(\left[\mathbf{1}_{Z}\right]\right)\right)\right)$, hence $\iota\left(f_{!}(\varphi)\right)=\iota\left(f_{!}\left(\varphi^{\prime}\right)\right)$.

13.1.2. Lemma. - We use notation from section 5. Let $\varphi$ and $\varphi^{\prime}$ be S-integrable functions in $\mathscr{C}_{+}(S[0,0, r])$. Assume $\varphi+\psi=\varphi^{\prime}+\psi$ for some function $\psi$ in $\mathscr{C}_{+}(S[0,0, r])$. Then $\iota\left(\mu_{S}(\varphi)\right)=\iota\left(\mu_{S}\left(\varphi^{\prime}\right)\right)$.

Proof. - By Propositions 5.7.2 and 5.8.4, it is enough to consider the case $r=1$. The result being clear if $\psi$ is $S$-integrable, it is enough to prove we may replace $\psi$ by some other function $\psi^{\prime}$ in $\mathscr{C}_{+}(S[0,0,1])$ which is $S$-integrable. We write $\psi=\sum_{i} c_{i} \psi_{i}$, with $c_{i}$ in $\mathscr{C}_{+}(S)$ and $\psi_{i}$ in $\mathcal{P}_{+}(S[0,0,1])$, and similarly for $\varphi$ and $\varphi^{\prime}$. We may write $\psi_{i}=\sum_{j} v_{i j}\left(\prod_{\ell} \alpha_{i, j, \ell}\right) \mathbb{L}^{\delta_{i, j}}$, with $\alpha_{i, j, \ell}$ and $\delta_{i, j}$ definable morphisms $S[0,0,1] \rightarrow \mathbb{Z}$ and the $v_{i j}$ in the ring $\mathbb{A}$, and similarly for the terms occurring in $\varphi$ and $\varphi^{\prime}$. We may suppose that the $\alpha_{i, j, \ell}$ take values in $\mathbb{N}$, and, by Presburger cell decomposition Theorem 4.3.2, we may assume that all functions $\alpha_{i, j, \ell}$ and $\delta_{i, j}$ have their support in some $S$-cell $Z$ and that they are $S$-linear, that is, of the form (4.1.1), and similarly for the analogue definable morphisms $S[0,0,1] \rightarrow \mathbb{N}$ occurring in the descriptions of $\varphi$ and $\varphi^{\prime}$. Replacing $\psi$ by $\psi+\tilde{\psi}$ for some positive $\tilde{\psi}$, we may furthermore suppose that all the $v_{i j}$ are in $\mathbb{A}_{+}$, where $\mathbb{A}$ and $\mathbb{A}_{+}$are the ring and semiring defined in 4.2. If the fibers of $Z$ above $S$ are all finite, every function on $Z$ is $S$-integrable, and there is nothing to do. Suppose thus that $Z$ is a (1)-cell and that all fibers of $Z$ above $S$ are infinite. By partitioning further we may assume that $Z \subset S \times \mathbb{N}$. Regrouping terms, the equality $\varphi+\psi=\varphi^{\prime}+\psi$ can now be rewritten as an equality 
between two (positive) sums of terms of the form

$$
w\left(\prod_{\ell} \alpha_{\ell}\right) \mathbb{L}^{\delta}
$$

with the $\alpha_{\ell}: Z \rightarrow \mathbb{N}$ and $\delta: Z \rightarrow \mathbb{Z} S$-linear definable morphisms and $w$ in the semiring $\mathbb{A}_{+}$. Writing $\delta(s, x)=a\left(\frac{x-c}{n}\right)+\gamma(s)$ with $n>0$ for $(s, x)$ on $Z$, the integrability of (13.1.1) only depends on the integer coefficient $a$, namely, it is integrable if and only if $a<0$. In the equality between a sum of terms (13.1.1), one can ignore all terms with $a \geq 0$ to obtain a new equality, because there can be no nontrivial relation between the terms with $a<0$ and those with $a \geq 0$. By Proposition 5.8.1 we may suppose that the terms coming from $\varphi$ and $\varphi^{\prime}$ are all integrable. Define $\psi_{i}^{\prime}:=\sum_{j \in J} v_{i j}\left(\prod_{\ell} \alpha_{i, j, \ell}\right) \mathbb{L}^{\delta_{i, j}}$, where $J$ consists of those $j$ for which $\mathbb{L}^{\delta_{i, j}}$ is $S$-integrable, and set $\psi^{\prime}:=\sum_{i} c_{i} \psi_{i}^{\prime}$. Then, $\psi^{\prime}$ is positive, $S$-integrable, and $\varphi+\psi^{\prime}=\varphi^{\prime}+\psi^{\prime}$, which finishes the proof.

13.2. - Fix $S$ in $\operatorname{Def}_{k}$. Let $X$ be in $\operatorname{Def}_{S}$. We shall say a Function $\varphi$ in $C(X)$ is $S$-integrable if it may be written as

$$
\varphi=\iota\left(\varphi_{+}\right)-\iota\left(\varphi_{-}\right),
$$

with $\varphi_{+}$and $\varphi_{-}$both $S$-integrable Functions in $C_{+}(X)$. We denote by $\mathrm{I}_{S} C(X)$ the graded subgroup of $C(X)$ consisting of $S$-integrable Functions. If $f: X \rightarrow Y$ is a morphism in $\operatorname{Def}_{S}$ and $\varphi$ is in $\mathrm{I}_{S} C(X)$, we set

$$
f_{!}(\varphi)=\iota\left(f_{!}\left(\varphi_{+}\right)\right)-\iota\left(f_{!}\left(\varphi_{-}\right)\right),
$$

with $\varphi_{+}$and $\varphi_{-}$in $\mathrm{I}_{S} C_{+}(X)$ satisfying (13.2.1). By Proposition 13.1.1, this is independent of the choice of $\varphi_{+}$and $\varphi_{-}$. We define in this way a morphism of abelian groups

$$
f_{!}: \mathrm{I}_{S} C(X) \longrightarrow \mathrm{I}_{S} C(Y) \text {. }
$$

Furthermore, if $g: Y \rightarrow Z$ is another morphism in $\operatorname{Def}_{S},(g \circ f)_{!}=g_{!} \circ f_{!}$. When $f$ is the morphism to $h_{\mathrm{Spec}(k)}$, we write $\mu(\varphi)$ for the element $f_{!}(\varphi)$ in $\mathscr{C}\left(h_{\mathrm{Spec}(k)}\right)$.

13.2.1. Proposition. - The following properties for $f_{!}$hold:

(1) Additivity and compatibility with inclusions: Axioms A2 and A4 of Theorem 10.1.1 are satisfied if one replaces $C_{+}$and $\mathscr{C}_{+}$by $C$ and $\mathscr{C}$, respectively.

(2) Projection formula: If $f: X \rightarrow S$ is a morphism in $\operatorname{Def}_{k}, \alpha$ is in $\mathscr{C}(S)$ and $\beta$ is in $\mathrm{I}_{S} C(X)$, then $f^{*}(\alpha) \beta$ is $S$-integrable and

$$
f_{!}\left(f^{*}(\alpha \beta)\right)=\alpha f_{!}(\beta) .
$$

(3) Let $\pi$ be the projection $\pi: S[0, n, 0] \rightarrow S$ with $S$ in $\operatorname{Def}_{k}$. Let $\varphi$ be in $\mathscr{C}(S[0, n, 0])$. Then $[\varphi]$ is $S$-integrable and $\pi_{!}([\varphi])=\left[\pi_{!}(\varphi)\right]$, $\pi_{!}$being defined as in 5.6 .

(4) Let $\pi$ be the projection $\pi: S[0,0, n] \rightarrow S$ with $S$ in $\operatorname{Def}_{k}$. Let $\varphi$ be in $\mathscr{C}(S[0,0, n])$. Then $[\varphi]$ is $S$-integrable if and only if there is a function $\varphi^{\prime}$ in $\mathscr{C}(S[0,0, n])$ with $\left[\varphi^{\prime}\right]=[\varphi]$ such that $\varphi^{\prime}$ is $\pi$-integrable in the sense of $[5.7$. Furthermore, when this holds, $\pi_{!}([\varphi])=\left[\mu_{S}\left(\varphi^{\prime}\right)\right]$. 
Proof. - The first three assertions follow directly from the corresponding statements for positive Functions. The last one follows directly from Lemma 13.1.2.

The following statement is a direct consequence of Theorem 12.1.1.

13.2.2. Theorem (Change of variable formula). — Let $f: X \rightarrow Y$ be a definable isomorphism between definable subassignments of $K$-dimension $d$. Let $\varphi$ be in $\mathscr{C}^{\leq d}(Y)$ having a non zero class $[\varphi]$ in $C^{d}(Y)$. Then $\left[f^{*}(\varphi)\right]$ is in $\mathrm{I}_{Y} C^{d}(X)$ and

$$
f_{!}\left(\left[f^{*}(\varphi)\right]\right)=\mathbb{L}^{(\operatorname{ordjac} f) \circ f^{-1}}[\varphi] .
$$

\section{Integrals with parameters}

Recall that, until the end of this section, all definable subassignments belong to $\operatorname{Def}_{k}$.

14.1. - In this section we consider the relative version of Theorem 10.1.1. By this we mean the construction of a theory for integrals with parameters in a definable subassignment $\Lambda$. One of the great advantages of our proof of Theorem 10.1.1 is that it carries literally to the relative case.

Let us fix $\Lambda$ in $\operatorname{Def}_{k}$. We introduce the subcategory $\operatorname{Def}_{\Lambda}^{\prime}$ of Def ${ }_{\Lambda}$ whose objects are definable subassigments $S$ of some $\Lambda[m, n, r]$, the morphism $p: S \rightarrow \Lambda$ being induced by the projection to $\Lambda$. For a given $S$ in $\operatorname{Def}_{\Lambda}^{\prime}$, we denote by $\operatorname{Def}_{S, \Lambda}^{\prime}$ the category whose objects are morphisms $Z \rightarrow S$ in $\operatorname{Def}_{\Lambda}^{\prime}$. To any object $f: S \rightarrow \Lambda$ in $\operatorname{Def}_{\Lambda}$ one may assign its graph $\Gamma_{f} \rightarrow \Lambda$ in $\operatorname{Def}_{\Lambda}^{\prime}$. This yields a functor $\Gamma: \operatorname{Def}_{\Lambda} \rightarrow \operatorname{Def}_{\Lambda}^{\prime}$ which is quasi-inverse to the inclusion functor $\operatorname{Def}_{\Lambda}^{\prime} \rightarrow \operatorname{Def}_{\Lambda}$ leading to an equivalence of categories between $\operatorname{Def}_{\Lambda}^{\prime}$ and $\operatorname{Def}_{\Lambda}$. More generally the functor $\Gamma$ induces an equivalence of categories $\Phi$ between $\operatorname{Def}_{S}$ and $\operatorname{Def}_{\Gamma(S), \Lambda}^{\prime}$, for every $S$ in $\operatorname{Def}_{\Lambda}$; this equivalence is compatible with $\operatorname{ordjac}_{\Lambda}$.

14.1.1. Theorem. - Let $\Lambda$ be in $\operatorname{Def}_{k}$. Let $S$ be in $\operatorname{Def}_{\Lambda}$, resp. in $\operatorname{Def}_{\Lambda}^{\prime}$. There is a unique functor from the category $\operatorname{Def}_{S}$, resp. Def $_{S, \Lambda}^{\prime}$, to the category of abelian semigroups, $Z \mapsto \mathrm{I}_{S} C_{+}(Z \rightarrow \Lambda)$, assigning to every morphism $f: Z \rightarrow Y$ in $\operatorname{Def}_{S}$, resp. in $\operatorname{Def}_{S, \Lambda}^{\prime}$, a morphism $f_{! \Lambda}: \mathrm{I}_{S} C_{+}(Z \rightarrow \Lambda) \rightarrow \mathrm{I}_{S} C_{+}(Y \rightarrow \Lambda)$ and satisfying the axioms similar to $\mathrm{A} 0-\mathrm{A} 8$ of Theorem 10.1.1 replacing $\mathrm{I}_{S} C_{+}\left({ }_{-}\right)$by $\mathrm{I}_{S} C_{+}\left({ }_{-} \rightarrow \Lambda\right)$ with the following changes:

In $\mathrm{A} 0(\mathrm{~b}) \lambda$ should be a morphism in $\mathrm{Def}_{\lambda}$, resp. in $\mathrm{Def}_{\Lambda}^{\prime}$. In $\mathrm{A}^{\prime}$, one should replace the function ordjac by the relative function $\operatorname{ordjac}_{\Lambda}$, as defined in 8.5 .

Furthermore, the relative analogue of Theorem 12.1.1, with ordjac $_{\Lambda}$ instead of ordjac, also holds, and the constructions are compatible with the equivalence of categories $\Phi$ between $\operatorname{Def}_{S}$ and $\operatorname{Def}_{\Gamma(S), \Lambda}^{\prime}$.

Proof. - It is enough to prove the Theorem in the relative setting, i.e., in the $\operatorname{Def}_{\Lambda}^{\prime}$ setting. The non relative case follows by using the equivalence $\Phi$ which is compatible with $\operatorname{ordjac}_{\Lambda}$, see also the end of 8.5. Our proofs of Theorem 10.1.1 and of Theorem 12.1.1 in the absolute setting has been designed in order to generalize verbatim to 
the present relative setting, with the following changes: replace everywhere absolute dimensions by relative dimensions; replace everywhere ordjac by its relative analogue $\operatorname{ordjac}_{\Lambda}$.

14.2. - When $\pi: Z \rightarrow \Lambda$ is the morphism to the final object in Def D $_{\Lambda}$, we write $\mathrm{I} C_{+}(Z \rightarrow \Lambda)$ instead of $\mathrm{I}_{\Lambda} C_{+}(Z \rightarrow \Lambda)$. We also denote by $\mu_{\Lambda}$ the morphism

$$
\pi_{! \Lambda}: \mathrm{I} C_{+}(Z \rightarrow \Lambda) \rightarrow C_{+}(\Lambda \rightarrow \Lambda)=\mathscr{C}_{+}(\Lambda)
$$

We call it the relative motivic measure. By Corollary 14.2.2 it corresponds to integrating along the fibers of $\Lambda$. One should remark that the notation is compatible with the one introduced in 5.7 and 9 .

Let $Z$ be in $\operatorname{Def}_{\Lambda}$. For every point $\lambda$ of $\Lambda$, we denote by $Z_{\lambda}$ the fiber of $Z$ at $\lambda$, as defined in 2.6. We have a natural restriction morphism $i_{\lambda}^{*}: C_{+}(Z \rightarrow \Lambda) \rightarrow C_{+}\left(Z_{\lambda}\right)$, which respects the grading.

14.2.1. Proposition. - Let $f: Z \rightarrow Y$ be a morphism in $\operatorname{Def}_{\Lambda}$. Let $\varphi$ be a Function in $C_{+}(Z \rightarrow \Lambda)$. Then $\varphi$ is $f$-integrable if and only if, for every point $\lambda$ of $\Lambda, i_{\lambda}^{*}(\varphi)$ is $f_{\lambda}$-integrable. Furthermore, when these conditions hold, then

$$
i_{\lambda}^{*}\left(f_{! \Lambda}(\varphi)\right)=f_{\lambda !}\left(i_{\lambda}^{*}(\varphi)\right)
$$

for every point $\lambda$ of $\Lambda$, where $f_{\lambda}: Z_{\lambda} \rightarrow Y_{\lambda}$ is the restriction of $f$ to the fiber $Z_{\lambda}$.

Proof. - It is enough to prove the statement for injections and projections. The case of injections being clear let us consider that of projections. It is enough to consider the case of projections along one sort of variables and the only case which is not a priori clear is that of a projection $Z=Y[0,0, r] \rightarrow Y$ which follows directly from Corollary 5.8.2.

In particular we have the following:

14.2.2. Corollary. - Let $f: Z \rightarrow \Lambda$ be in $\operatorname{Def}_{\Lambda}$. Let $\varphi$ be a Function in $C_{+}(Z \rightarrow$ $\Lambda)$. Then $\varphi$ is integrable if and only if for every point $\lambda$ of $\Lambda, i_{\lambda}^{*}(\varphi)$ is in $\mathrm{I}_{+}\left(Z_{\lambda}\right)$. If these conditions hold, then

$$
i_{\lambda}^{*}\left(\mu_{\Lambda}(\varphi)\right)=\mu_{\lambda}\left(i_{\lambda}^{*}(\varphi)\right)
$$

for every point $\lambda$ of $\Lambda$, where $\mu_{\lambda}$ denotes the motivic measure on $\operatorname{Def}_{k(\lambda)}$.

It is not clear whether the if and only if statement of Proposition 14.2.1 and its corollary hold for $\varphi$ in $C(Z \rightarrow \Lambda)$.

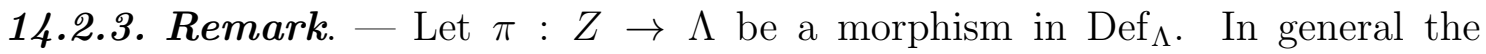
elements $\mu_{\Lambda}(\varphi)$ and $\pi_{!}(\varphi)$ may be quite different. For instance, assume that $\pi$ is an isomorphism, then $\mu_{\Lambda}\left(\left[\mathbf{1}_{Z}\right]\right)=\mathbf{1}_{\Lambda}$, while $\pi_{!}\left(\left[\mathbf{1}_{Z}\right]\right)=\mathbb{L}^{\text {ordjac } \pi \circ \pi^{-1}}\left[\mathbf{1}_{\Lambda}\right]$. One should remark that in this case $\left[\mathbf{1}_{Z}\right]$ is of degree 0 in $C_{+}(Z \rightarrow \Lambda)$, since the relative $K$ dimension is 0 , while $\left[\mathbf{1}_{Z}\right]$ is of maximal degree in $C_{+}(Z)$. Of course, if $\Lambda$ is a subassigment of $h_{\mathbb{A}_{k}^{m} \times \mathbb{Z}^{r}}$ then $\mu_{\Lambda}(\varphi)=\pi_{!}(\varphi)$ whenever the integrability conditions 
are met. Also, if we have a morphism $\pi^{\prime}: \Lambda \rightarrow T$ in $\operatorname{Def}_{k}$, in general $\mu_{T}\left(\left[\mu_{\Lambda}(\varphi)\right]\right) \neq$ $\mu_{T}(\varphi)$.

14.3. - Let $X$ be in $\operatorname{Def}_{\Lambda}$. The canonical morphism $\iota: \mathscr{C}_{+}(X) \rightarrow \mathscr{C}(X)$ induces a morphism $\iota: C_{+}(X \rightarrow \Lambda) \rightarrow C(X \rightarrow \Lambda)$ for which the analogue of Proposition 13.1.1 holds. This allows us, for $S$ in $\operatorname{Def}_{\Lambda}$ and $X$ in $\operatorname{Def}_{S}$, to define $\mathrm{I}_{S} C(X \rightarrow \Lambda)$, and for $f: X \rightarrow Y$ a morphism in Def $_{S}$, to define $f_{! \Lambda}: \mathrm{I}_{S} C(X \rightarrow \Lambda) \rightarrow \mathrm{I}_{S} C(Y \rightarrow \Lambda)$ and $\mu_{\Lambda}: \mathrm{I} C(X \rightarrow \Lambda) \rightarrow \mathscr{C}(\Lambda)$ as in 13.2 .

The relative analogues of Proposition 13.2 .1 and Theorem 13.2 .2 hold in this setting with similar proofs.

14.4. Rationality theorems. - Now we can state the following general rationality theorem.

14.4.1. Theorem. - Let $\pi: Z \rightarrow \Lambda \times \mathbb{N}^{r}$ be a morphism in $\operatorname{Def}_{k}, \mathbb{N}^{r}$ being considered as a definable subassignment of $h_{\mathbb{Z}^{r}}$. For every $\varphi$ in $\operatorname{IC}\left(Z \rightarrow \Lambda \times \mathbb{N}^{r}\right)$, the Poincaré series

$$
P_{\varphi, \pi}(T):=\sum_{n \in \mathbb{N}^{r}} \mu_{\Lambda}\left(\varphi_{\mid \pi^{-1}(\Lambda \times\{n\})}\right) T^{n}
$$

belongs to $\mathscr{C}(\Lambda) \llbracket T_{1}, \cdots, T_{r} \rrbracket_{\Gamma}$, where $\varphi_{\mid \pi^{-1}(\Lambda \times\{n\})}$ is considered as an element of $I C(Z \rightarrow \Lambda)$ and $\mu_{\Lambda}\left(\varphi_{\mid \pi^{-1}(\Lambda \times\{n\})}\right)$ as an element of $\mathscr{C}(\Lambda)$, and where $\mathscr{C}(\Lambda) \llbracket T_{1}, \cdots, T_{r} \rrbracket_{\Gamma}$ is as in 5.7 .

Proof. - By construction the function $\Phi:=\mu_{\Lambda \times \mathbb{N}^{r}}(\varphi)$ belongs to $\mathscr{C}\left(\Lambda \times \mathbb{N}^{r}\right)$. By Proposition 14.2.1, its restriction $\Phi_{n}$ to $\mathscr{C}(\Lambda \times\{n\})$ satisfies $\Phi_{n}=\mu_{\Lambda}\left(\varphi_{\mid \pi^{-1}(\Lambda \times\{n\})}\right)$, hence the result follows from Theorem 5.7.1.

Let us give an example of application of the above result. Let $g: X \rightarrow \Lambda$ be a morphism in $\operatorname{Def}_{k}$, and consider a morphism $f: X \rightarrow h_{\mathbb{A}_{k(t))}^{1}}$. For $n \geq 1$, we denote by $\mathcal{X}_{n}$ the definable subassignment of $X$ defined by

$$
\mathcal{X}_{n}(K)=\{x \in X(K) \mid \text { ord } f(x)=n\},
$$

for $K$ a field containing $k$. We denote by $f_{n}: \mathcal{X}_{n} \rightarrow h_{\mathbb{G}_{m, k}}$ the morphism given by $x \mapsto \overline{\mathrm{ac}}(f(x))$. By taking the product of morphisms $g$ and $f_{n}$ we get a morphism $\mathcal{X}_{n} \rightarrow \Lambda \times h_{\mathbb{G}_{m, k}}$. Here $\mathbb{G}_{m, k}$ is $\mathbb{A}_{k}^{1} \backslash\{0\}$, the affine line minus the origin. For $\varphi$ in $\mathrm{I} C(X \rightarrow \Lambda)$ we consider the generating series

$$
P_{f, \varphi}(T):=\sum_{n>0} \mu_{\Lambda \times h_{\mathbb{G}_{m, k}}}\left(\varphi_{\mid \mathcal{X}_{n}}\right) T^{n} .
$$

By Theorem 14.4.1, $P_{f, \varphi}(T)$ belongs to $\mathscr{C}\left(\Lambda \times h_{\mathbb{G}_{m, k}}\right) \llbracket T \rrbracket_{\Gamma}$, hence is a rational series in $T$. This example encompasses the motivic analogues of rationality results for $p$-adic Igusa and Serre series (cf. [11] and [12]) in [14] and [15. Motivic analogues of analytic $p$-adic Igusa and Serre series have been studied by J. Sebag [34]. 
14.5. Application to ramification. - In this section we shall apply the preceding results to the study of the behaviour of the motivic measure under the ramification $t \mapsto t^{1 / e}$, when the coefficients in the value field sort are restricted to $k[t]$. We use the observation that the purely ramified field extension of degree $e$ of $k((t))$ is isomorphic to $k((t))$. We still assume the language is $\mathcal{L}_{\mathrm{DP}, \mathrm{P}}$. If $\varphi$ is a formula with coefficients in $k[t]$ in the valued field sort and coefficients in $k$ in the residue field sort, with $m$ free variables in the valued field sort, $n$ in the residue field sort and $r$ in the value group sort and $e$ is an integer $\geq 1$, we denote by $\varphi^{(e)}$ the formula obtained by replacing $t$ by $t^{e}$ in every occurrence of $t$ in $\varphi$. For instance, if $\varphi$ is the formula $\exists x \operatorname{ord}\left(t y+t^{3}-x^{5}\right) \geq 2, \varphi^{(e)}$ is the formula $\exists x \operatorname{ord}\left(t^{e} y+t^{3 e}-x^{5}\right) \geq 2$. We denote by $Z^{(e)}=Z_{\varphi}^{(e)}$ the subassignment defined by $\varphi^{(e)}$. Hence, to the single formula $\varphi$ we may associate the family $\left(Z_{\varphi}^{(e)}\right), e \in \mathbb{N}_{>0}$, of definable subassignments of $h[m, n, r]$. We call such a family the $(e)$-family of definable subassignments defined by $\varphi$. A family of morphisms $f^{(e)}: Z^{(e)} \rightarrow Y^{(e)}$ will be called a morphism between $(e)$-families $Z^{(e)}$ and $Y^{(e)}$ if the family $\operatorname{Graph} f^{(e)}$ is an $(e)$-family of definable subassignments. We denote by $\pi^{(e)}: Z \rightarrow \Lambda=h[0, n, r]$ the projection onto the last factors. We also consider a morphism of $(e)$-families $\alpha^{(e)}: Z^{(e)} \rightarrow h_{\mathbb{Z}}^{(e)}=h_{\mathbb{Z}}$ defined by some formula $\psi$.

14.5.1. Proposition. - Use the above notation, in particular, $\Lambda=h[0, n, r]$ and assume that the coefficients of $\varphi$ and $\psi$ in the valued field sort all belong to $k[t]$. Assume also that all morphisms $\alpha^{(e)}$ take their values in $\mathbb{N}$ and that for each $\lambda$ in $\Lambda$ the fibers $\left(Z^{(e)}\right)_{\lambda}$ are bounded as in 12.2. Then, for every e, $\left[\mathbf{1}_{Z^{(e)}} \mathbb{L}^{-\alpha^{(e)}}\right]$ belongs to $\mathrm{I} C_{+}\left(Z^{(e)} \rightarrow \Lambda\right)$ and there is a function $\Phi$ in $\mathscr{C}_{+}\left(\mathbb{N}_{>0} \times \Lambda\right)$ such that $\Phi_{\mid\{e\} \times \Lambda}$ coincides with $\mu_{\Lambda}\left(\left[\mathbf{1}_{Z^{(e)}} \mathbb{L}^{-\alpha^{(e)}}\right]\right)$ for every $e>0$. Here we view $\mathbb{N}_{>0} \times \Lambda$ as a definable subassignment of $h_{\mathbb{Z}} \times \Lambda$.

Proof. - The fact that $\left[\mathbf{1}_{Z^{(e)}} \mathbb{L}^{-\alpha^{(e)}}\right]$ belongs to $\mathrm{I}_{+}\left(Z^{(e)} \rightarrow \Lambda\right)$ for every $e$ follows from Proposition 12.2.2 and Proposition 14.2.1. We introduce an additional variable $\vartheta$ in the valued field sort and replace every occurrence of $t$ in $\varphi$ and $\psi$ by $\vartheta$, to get formulas $\tilde{\varphi}$ and $\tilde{\psi}$. The formula $\tilde{\varphi}$ defines a definable subassignment $\tilde{Z}$ of $h[m+1, n, r]$. We set $\tilde{\Lambda}:=\Lambda[1,0,0]$ and denote by $\tilde{\pi}$ the projection $\tilde{Z} \rightarrow \tilde{\Lambda}$. Similarly $\tilde{\psi}$ defines a morphism $\tilde{\alpha}: \tilde{Z} \rightarrow h_{\mathbb{Z}}$. It follows again from Proposition 12.2 .2 and Proposition 14.2 .1 that $\left[\mathbf{1}_{\tilde{Z}} \mathbb{L}^{-\tilde{\alpha}}\right]$ belongs to $\mathrm{I} C_{+}(\tilde{Z} \rightarrow \tilde{\Lambda})$. Hence, by 14.2 , we may set $\Theta:=\mu_{\tilde{\Lambda}}\left(\left[\mathbf{1}_{\tilde{Z}} \mathbb{L}^{-\tilde{\alpha}}\right]\right)$ in $\mathscr{C}(\tilde{\Lambda})$. By construction, for every $e$, $i_{\vartheta=t^{e}}^{*}(\Theta)=\mu_{\Lambda}\left(\left[\mathbf{1}_{Z^{(e)}} \mathbb{L}^{-\alpha^{(e)}}\right]\right)$, where $i_{\vartheta=t^{e}}^{*}$ denotes the fiber morphism at $t^{e}$ under the projection $\tilde{\Lambda} \rightarrow h[1,0,0]$, cf. the proof of [14.2.1. Hence, the statement follows from Lemma 14.5.2, which is easily proved, using cell decomposition.

14.5.2. Lemma. - Let $\Lambda$ be $h[0, n, r]$ and set $\tilde{\Lambda}:=\Lambda[1,0,0]$. Let $\Theta$ belong to $\mathscr{C}_{+}(\tilde{\Lambda})$. Then there exists a unique function $\Phi$ in $\mathscr{C}_{+}\left(\mathbb{N}_{>0} \times \Lambda\right)$ such that $\Phi_{\mid\{e\} \times \Lambda}$ coincides with $i_{\vartheta=t^{e}}^{*}(\Theta)$ for every $e>0$, where $i_{\vartheta=t^{e}}^{*}$ denotes the fiber morphism at $t^{e}$ under the projection $\tilde{\Lambda} \rightarrow h[1,0,0]$. 
Proof. - Apply cell decomposition to obtain cells adapted to $\Theta$, say, with cells having centers $c_{i}$ and base $C_{i}$. For every field $K$ containing $k$, the sets $c_{i}\left(C_{i}\right)(K)$ are finite. By dimension theory, there exists a polynomial $g$ in $k((t))\left[x_{1}\right]$, independent of $K$ and $i$, which vanishes at all points of $c_{i}\left(C_{i}\right)(K)$ for all $K$ and all $i$. Hence, $\cup_{K}\left\{\operatorname{ord}\left(c_{i}\left(C_{i}\right)(K)\right)\right\}$ is a finite set of integers. The condition on $n \geq 0$ and on $\lambda$ in $\Lambda$ that a value $\left(\lambda, t^{n}\right)$ lies in a given cell is thus easily checked, by the nature of cell conditions, to be a definable condition.

14.5.3. Theorem. - Assume the notation and assumptions of Proposition 14.5.1. Then the series

$$
\sum_{e>0} \mu_{\Lambda}\left(\iota\left(\left[\mathbf{1}_{Z^{(e)}} \mathbb{L}^{-\alpha^{(e)}}\right]\right)\right) T^{e}
$$

belongs to $\mathscr{C}(\Lambda) \llbracket T \rrbracket_{\Gamma}$, where $\iota: \mathscr{C}_{+}(\Lambda) \rightarrow \mathscr{C}(\Lambda)$ is the natural map, and where $\mathscr{C}(\Lambda) \llbracket T \rrbracket_{\Gamma}$ is as in 5.7 .

Proof. — Follows directly from Proposition 14.5.1 and Theorem 14.4.1.

14.5.4. Remark. - The trick of adding a new variable to prove Theorem 14.5.3 (cf. the proof of Proposition 14.5.1) was indicated to us by Jan Denef.

\section{III}

\section{INTEGRATION ON VARIETIES AND COMPARISON THEOREMS}

\section{Integration on varieties and Fubini Theorem}

15.1. Integrable volume forms. - Let $S$ be a definable subassignment of $h[m, n, r]$ of $K$-dimension $d$. We shall consider the canonical volume form $\left|\omega_{0}\right|_{S}$ in $|\tilde{\Omega}|_{+}(S)$, which was introduced in Definition-Lemma 8.3.1. We shall also consider the image of $\left|\omega_{0}\right|_{S}$ in $|\tilde{\Omega}|(S)$, which we shall also denote by $\left|\omega_{0}\right|_{S}$. Let $\alpha$ be in $|\tilde{\Omega}|_{+}(S)$, resp. in $|\tilde{\Omega}|(S)$. There exists a unique Function $\psi_{\alpha}$ in $C_{+}^{d}(S)$, resp. in $C^{d}(S)$, such that $\alpha=\psi_{\alpha}\left|\omega_{0}\right|_{S}$ in $|\tilde{\Omega}|_{+}(S)$, resp. in $|\tilde{\Omega}|(S)$. We shall say $\alpha$ is integrable when $\psi_{\alpha}$ is integrable and then set

$$
\int_{S} \alpha:=\mu\left(\psi_{\alpha}\right)
$$

in $\mathscr{C}_{+}\left(h_{\mathrm{Spec}(k)}\right)$, resp. in $\mathscr{C}\left(h_{\mathrm{Spec}(k)}\right)$.

More generally, if $f: S \rightarrow S^{\prime}$ is a morphism in $\operatorname{Def}_{k}$ such that $S$ and $S^{\prime}$ have respectively dimension $s$ and $s^{\prime}$, we say $\alpha$ in $|\tilde{\Omega}|_{+}(S)$ is $f$-integrable if $\psi_{\alpha}$ is $f$ integrable and then set

$$
f_{!}^{\text {top }}(\alpha):=\left\{f_{!}\left(\psi_{\alpha}\right)\right\}_{s^{\prime}}\left|\omega_{0}\right|_{S^{\prime}}
$$


where $\left\{f_{!}\left(\psi_{\alpha}\right)\right\}_{s^{\prime}}$ denotes the component of $f_{!}\left(\psi_{\alpha}\right)$ in $C_{+}^{s^{\prime}}\left(S^{\prime}\right)$ (the top dimensional component). Let us denote by $\mathrm{I}_{f}|\tilde{\Omega}|_{+}(S)$ the set of $f$-integrable positive volume forms. We have thus defined a canonical morphism

$$
f_{!}^{\text {top }}: \mathrm{I}_{f}|\tilde{\Omega}|_{+}(S) \longrightarrow|\tilde{\Omega}|_{+}\left(S^{\prime}\right) .
$$

When $S^{\prime}=h_{\text {Spec } k}$, one recovers the above construction.

Let us consider from now on varieties $\mathcal{X}$ and $\mathcal{X}^{\prime}$ over $k((t))$, and varieties $X$ and $X^{\prime}$ over $k$. We want to extend the above construction to the global setting where $f: S \rightarrow S^{\prime}$ is a morphism of definable subassignments with $S$ a definable subassignment of $h_{W}, W=\mathcal{X} \times X \times \mathbb{Z}^{r}$, and $S^{\prime}$ a definable subassignment of $h_{W^{\prime}}$, $W^{\prime}=\mathcal{X}^{\prime} \times X^{\prime} \times \mathbb{Z}^{r^{\prime}}$. We still assume that $S$ is of $K$-dimension $s$ and $S^{\prime}$ if of $K$-dimension $s^{\prime}$.

Let $U$ be an affine open in $W$, that is, a subset of the form $\mathcal{U} \times O \times \mathbb{Z}^{r}$ with $\mathcal{U}$ and $O$, respectively, affine open in $\mathcal{X}$ and $X$. There exists an isomorphism of varieties $\varphi: V \rightarrow U$ with $V$ affine open in $\mathbb{A}_{k((t))}^{m} \times \mathbb{A}_{k}^{n} \times \mathbb{Z}^{r}$ inducing the identity on the $\mathbb{Z}^{r}$-factor. Similarly, let $U^{\prime}$ be an affine open subset of $W^{\prime}$ and assume that $f\left(S \cap h_{U}\right) \subset S^{\prime} \cap h_{U^{\prime}}$. We denote by $f_{U}: S \cap h_{U} \rightarrow S^{\prime} \cap h_{U^{\prime}}$ the morphism induced by $f$. Choose an isomorphism of varieties $\varphi^{\prime}: V^{\prime} \rightarrow U^{\prime}$ with $V^{\prime}$ affine open in $\mathbb{A}_{k((t))}^{m^{\prime}} \times \mathbb{A}_{k}^{n^{\prime}} \times \mathbb{Z}^{r^{\prime}}$, inducing the identity on the $\mathbb{Z}^{r^{\prime}}$-factor. We denote by $\tilde{\varphi}$ and $\tilde{\varphi}^{\prime}$ the restriction of $\varphi$ and $\varphi^{\prime}$ to $\varphi^{-1}\left(S \cap h_{U}\right)$ and $\varphi^{\prime-1}\left(S^{\prime} \cap h_{U^{\prime}}\right)$, respectively, and by $\tilde{f}_{U}: \varphi^{-1}\left(S \cap h_{U}\right) \rightarrow \varphi^{\prime-1}\left(S^{\prime} \cap h_{U^{\prime}}\right)$ the morphism such that $f_{U} \circ \tilde{\varphi}=\tilde{\varphi}^{\prime} \circ \tilde{f}_{U}$. We shall say $\alpha$ in $|\tilde{\Omega}|_{+}\left(S \cap h_{U}\right)$ if $f_{U^{-}}$-integrable if $\tilde{\varphi}^{*}(\alpha)$ is $\tilde{f}_{U^{-}}$-integrable, and we then define $f_{U !}^{\text {top }}(\alpha)$ by the formula

$$
\left(\tilde{\varphi}^{\prime}\right)^{*}\left(f_{U !}^{\text {top }}(\alpha)\right)=\tilde{f}_{U !}^{\text {top }}\left(\tilde{\varphi}^{*}(\alpha)\right)
$$

which makes sense since $\left(\tilde{\varphi}^{\prime}\right)^{*}$ yields an isomorphism between $|\tilde{\Omega}|_{+}\left(S^{\prime} \cap h_{U^{\prime}}\right)$ and $|\tilde{\Omega}|_{+}\left(\varphi^{\prime-1}\left(S^{\prime} \cap h_{U^{\prime}}\right)\right)$. It follows directly from Lemma 15.1.1 that this definition does not depend on the choice of $\varphi$ and $\varphi^{\prime}$.

15.1.1. Lemma. - Let $f: S \rightarrow S^{\prime}$ be a morphism in $\operatorname{Def}_{k}$. Consider a commutative diagram

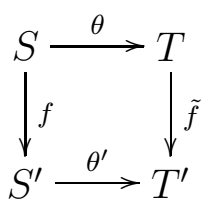

in $\operatorname{Def}_{k}$, with $\theta$ and $\theta^{\prime}$ isomorphisms. Take $\alpha$ in $|\tilde{\Omega}|_{+}(T)$. Then $\alpha$ is $\tilde{f}$-integrable if and only if $\theta^{*}(\alpha)$ is $f$-integrable and then

$$
f_{!}^{\mathrm{top}}\left(\theta^{*}(\alpha)\right)=\theta^{*}\left(\tilde{f}_{!}^{\mathrm{top}}(\alpha)\right)
$$

Proof. - This follows directly from the fact that, on Functions, $\theta_{!}^{\prime} f_{!}=\tilde{f}_{!} \theta_{!}$together with Theorem 12.1.1 or Proposition 12.1.2, and from the observation that $\theta_{!} \theta^{*}(\alpha)=$ $\alpha$ and similarly for $\theta^{\prime}$. 
Now we can handle the general case. We shall say a positive volume form $\alpha$ in $|\tilde{\Omega}|_{+}(S)$ is $f$-integrable if for every affine open subset $U$ in $W$ and every affine open subset $U^{\prime}$ of $W^{\prime}$ such that $f\left(S \cap h_{U}\right) \subset S^{\prime} \cap h_{U^{\prime}}$, the restriction $\alpha_{\mid U}$ of $\alpha$ to $|\tilde{\Omega}|_{+}\left(S \cap h_{U}\right)$ is $f_{U^{-}}$integrable. If these conditions hold, we consider a finite covering of $W$ by affine open subsets $U_{i}, i \in J$, and a finite covering of $W^{\prime}$ by affine open subsets $U_{i}^{\prime}, i \in J$, such that $f\left(S \cap h_{U_{i}}\right) \subset S^{\prime} \cap h_{U_{i}^{\prime}}$, for every $i$ (such coverings always exist). Let $\left(S_{\ell}\right)_{\ell \in L}$ be a finite partition of $S$ into definable subassignments such that each $S_{\ell}$ is a definable subassignment of $h_{U_{i_{\ell}}}$ for some $i_{\ell}$. Set

$$
\alpha_{\ell}:=\left[\mathbf{1}_{S_{\ell}}\right] \alpha
$$

in $|\tilde{\Omega}|_{+}(S)$. Clearly

$$
\alpha=\sum_{\ell \in L} \alpha_{\ell}
$$

and it follows from the hypotheses that the restriction $\alpha_{\ell \mid U_{i_{\ell}}}$ of $\alpha_{\ell}$ to $|\tilde{\Omega}|_{+}\left(S \cap h_{U_{i_{\ell}}}\right)$ is $f_{U_{i_{\ell}}}$-integrable. Now we can set

$$
f_{!}^{\text {top }}(\alpha):=\sum_{\ell \in L} j_{+}\left(f_{U_{i_{\ell}} !}^{\text {top }}\left(\alpha_{\ell \mid U_{i_{\ell}}}\right)\right),
$$

where $j_{+}$denotes the morphism $|\tilde{\Omega}|_{+}\left(S^{\prime} \cap h_{U_{i_{\ell}}^{\prime}}\right) \rightarrow|\tilde{\Omega}|_{+}\left(S^{\prime}\right)$ which is the zero morphism if $S^{\prime} \cap h_{U_{i_{\ell}}^{\prime}}$ is of $K$-dimension $<s^{\prime}$, and is given by extension by zero if $S^{\prime} \cap h_{U_{i_{\ell}}^{\prime}}$ is of $K$-dimension $s^{\prime}$. By additivity this definition is independent of all choices we made.

Hence, if we denote by $\mathrm{I}_{f}|\tilde{\Omega}|_{+}(S)$ the set of $f$-integrable positive volume forms, we defined a morphism

$$
f_{!}^{\text {top }}: \mathrm{I}_{f}|\tilde{\Omega}|_{+}(S) \longrightarrow|\tilde{\Omega}|_{+}\left(S^{\prime}\right) .
$$

When $S^{\prime}=h_{\text {Spec } k}$, we shall say integrable for $f$-integrable and write $\int_{S} \alpha$ for $f_{!}^{\text {top }}(\alpha)$. In particular $\int_{S} \alpha$ lies in $\mathscr{C}_{+}\left(h_{\text {Spec } k}\right)$ and its definition is compatible with the beginning of this section.

All the above constructions carry over literally to $|\tilde{\Omega}|$ replacing everywhere $C_{+}$ by $C$ and $|\tilde{\Omega}|_{+}$by $|\tilde{\Omega}|$.

15.2. General Fubini Theorem for fiber integrals. - We can now state a general form of Fubini Theorem for motivic integration.

\subsubsection{Theorem (Fubini Theorem for fiber integrals)}

Let $f: S \rightarrow S^{\prime}$ be a morphism of definable subassignments with $S$ a definable subassignment of $h_{W}, W=\mathcal{X} \times X \times \mathbb{Z}^{r}$ and $S^{\prime}$ a definable subassignment of $h_{W^{\prime}}$, $W^{\prime}=\mathcal{X}^{\prime} \times X^{\prime} \times \mathbb{Z}^{r^{\prime}}$. Assume $S$ is of $K$-dimension $s, S^{\prime}$ if of $K$-dimension $s^{\prime}$ and that the fibers $S_{y}$ of $f$ are of dimension $d=s-s^{\prime}$ for all points $y$ in $S^{\prime}$.

(1) Let $\alpha$ be in $|\tilde{\Omega}|_{+}(S)$. Then $\alpha$ is integrable if and only if $\alpha$ is $f$-integrable and $f_{!}^{\text {top }}(\alpha)$ is integrable. 
(2) Let $\alpha$ be in $|\tilde{\Omega}|(S)$. If $\alpha$ is integrable, then $\alpha$ is $f$-integrable and $f_{!}^{\text {top }}(\alpha)$ is integrable.

(3) Let $\alpha$ be in $|\tilde{\Omega}|_{+}(S)$ or in $|\tilde{\Omega}|(S)$, and assume that $\alpha$ is integrable. Then

$$
\int_{S} \alpha=\int_{S^{\prime}} f_{!}^{\text {top }}(\alpha)
$$

Proof. - We may reduce to the case where $\mathcal{X}, X, \mathcal{X}^{\prime}$, and $X^{\prime}$ are all affine spaces. Let us consider the positive case. Note that if $\varphi$ is a $f$-integrable Function in $C_{+}^{s}(S)$, It follows from the hypothesis made on the dimension of the fibers of $f$, that $f_{!}(\varphi)$ lies in $C_{+}^{s^{\prime}}\left(S^{\prime}\right)$. So the result follows from A0, since $p_{!}=p_{!}^{\prime} \circ f_{!}$, where $p$ and $p^{\prime}$ denote respectively the projections of $S$ and $S^{\prime}$ onto $h_{\mathrm{Spec} k}$. The general case follows directly from the postive case.

15.3. A reformulation of the change of variable formula. - Let $f: S \rightarrow S^{\prime}$ be a morphism in GDef $_{k}$. Assume $S$ and $S^{\prime}$ are of $K$-dimension $s$.

15.3.1. Theorem. - Let $f: S \rightarrow S^{\prime}$ be a morphism of definable subassignments as above. Assume $f$ is an isomorphism of definable subassignments. A volume form $\alpha$ in $\left|\tilde{\Omega}\left(S^{\prime}\right)\right|_{+}$or in $\left|\tilde{\Omega}\left(S^{\prime}\right)\right|$ is integrable if and only if $f^{*}(\alpha)$ is integrable. When this holds, then

$$
\int_{S} f^{*}(\alpha)=\int_{S^{\prime}} \alpha
$$

Proof. - We reduce to the affine case where $S$ and $S^{\prime}$ are in $\operatorname{Def}_{k}$. By the very definition of ordjac $f$ we have

$$
f^{*}\left|\omega_{0}\right|_{S^{\prime}}=\mathbb{L}^{-\operatorname{ordjac} f}\left|\omega_{0}\right|_{S},
$$

and the result follows from the change of variable formula Theorem 12.1.1 and 13.2 .2 .

15.4. Leray residues. — We start by recalling the standard Leray residues of differential forms in the framework of $K((t))$-analytic manifolds with $K$ of characteristic zero, where the notion of $K((t))$-analytic manifolds is as in section 3 .

Let us consider a morphism $f: \mathcal{X} \rightarrow \mathcal{Y}$ of $K((t))$-analytic manifolds. Assume $\mathcal{X}$ is of dimension $r, \mathcal{Y}$ is of dimension $s$ and that for every point $y$ in $\mathcal{Y}$ the fiber $\mathcal{X}_{y}$ of $f$ at $y$ is nonempty and contains a dense open which is a submanifold of $\mathcal{X}$ of dimension $d=r-s$. Take a degree $r$ differential form $\omega_{\mathcal{X}}$ on $\mathcal{X}$ and a degree $s$ differential form $\omega_{\mathcal{y}}$ on $\mathcal{Y}$ which is non zero on a dense open subset. For $y$ in a dense open subset of $\mathcal{Y}$, we define a degree $d$ differential form $\left(\frac{\omega_{\mathcal{X}}}{\omega_{\mathcal{Y}}}\right)_{y}$ on a dense open subset of the fiber $\mathcal{X}_{y}$.

By working on charts, we only have to treat the local case, namely, when $\mathcal{X}$ is the affine manifold $K((t))^{r}$ with coordinates $x_{1}, \ldots, x_{r}, \mathcal{Y}$ is $K((t))^{s}$ with coordinates $y_{1}, \ldots, y_{s}, f$ is given by $s$ analytic maps $f_{1}, \ldots, f_{s}, \omega_{\mathcal{X}}=g d x_{1} \wedge \cdots \wedge d x_{r}$, and $\omega_{\mathcal{Y}}=h d y_{1} \wedge \cdots \wedge d y_{s}$, with $g, h$ analytic and $h$ nonzero on a dense open of $\mathcal{Y}$. For $I=\left\{i_{1}, \ldots, i_{d}\right\} \subset\{1, \ldots r\}, i_{1}<\cdots<i_{d}$, we denote by $\mathrm{Jac}_{I}$ the determinant of 
the matrix $\left(\frac{\partial f_{i}}{\partial x_{j}}\right)_{1 \leq i \leq s, j \in\{1, \ldots, r\} \backslash I}$. For each $y$ in a dense open of $\mathcal{Y}$, there exists $I$ such that $\mathrm{Jac}_{I}$ is nonzero at a dense open of the fiber $\mathcal{X}_{y}$. If then moreover $h(y) \neq 0$, we define the differential form $\left(\frac{\omega_{\mathcal{X}}}{\omega_{\mathcal{Y}}}\right)_{y}$ on a dense open of $\mathcal{X}_{y}$ to be the differential form

$$
\frac{\varepsilon g}{h(y) \operatorname{Jac}_{I}} d x_{i_{1}} \wedge \cdots \wedge d x_{i_{d}}
$$

where $\varepsilon= \pm 1$ is such that, on $\mathcal{X}$,

$$
d x_{j_{1}} \wedge \cdots \wedge d x_{j_{s}} \wedge d x_{i_{1}} \wedge \cdots \wedge d x_{i_{d}}=\varepsilon d x_{1} \wedge \cdots \wedge d x_{r}
$$

with $\left\{j_{1}, \ldots, j_{s}\right\}=\{1, \ldots, r\} \backslash I$ and $j_{1}<\cdots<j_{s}$. It is independent of the choice of $I$ at a dense open of $\mathcal{X}_{y}$.

Now we come to the definable setting. Let $f: S \rightarrow S^{\prime}$ be a morphism of definable subassignments with $S$ a definable subassignment of $h_{W}, W=\mathcal{X} \times X \times \mathbb{Z}^{r}$ and $S^{\prime}$ a definable subassignment of $h_{W^{\prime}}, W^{\prime}=\mathcal{X}^{\prime} \times X^{\prime} \times \mathbb{Z}^{r^{\prime}}$. Assume that $S$ is of $K$-dimension $s, S^{\prime}$ if of $K$-dimension $s^{\prime}$ and that the fibers $S_{y}$ of $f$ are of dimension $d=s-s^{\prime}$ for all $y$ in $S^{\prime}$. Take $\omega_{S}$ in $\tilde{\Omega}^{s}(S)$ and $\omega_{S}^{\prime}$ a generator of the $\mathcal{A}\left(S^{\prime}\right) / \mathcal{A}^{<}\left(S^{\prime}\right)$ module $\tilde{\Omega}^{s^{\prime}}\left(S^{\prime}\right)$. Proceeding as before, and using Theorem 3.2.1, one defines by the Leray residue construction an element $\left(\frac{\omega_{S}}{\omega_{S^{\prime}}}\right)_{y}$ in $\tilde{\Omega}^{s-s^{\prime}}\left(S_{y}\right)$, for every point $y$ in $S^{\prime}$ outside a definable subassignment of $K$-dimension $<s^{\prime}$. Consider now $\alpha$ in $|\tilde{\Omega}|_{+}(S)$ and $|\omega|$ a gauge form in $|\tilde{\Omega}|_{+}\left(S^{\prime}\right)$. If $\alpha$ is the class of $\left(\omega^{\prime}, g\right)$ and $|\omega|$ is the class of $(\omega, 1)$, we define $\left(\frac{\alpha}{|\omega|}\right)_{y}$ as the class of $\left(\left(\frac{\omega^{\prime}}{\omega}\right)_{y}, g\right)$ in $|\tilde{\Omega}|_{+}\left(S_{y}\right)$, for every point $y$ in $S^{\prime}$ outside a definable subassignment of $K$-dimension $<s^{\prime}$. The same construction may be similarly done for $|\tilde{\Omega}|$ instead of $|\tilde{\Omega}|_{+}$.

The following proposition shows that considering $f_{\text {! }}$ is essentially the same as taking fiber integrals of Leray residues of canonical volume forms. More precisely:

15.4.1. Proposition. - Let $f: S \rightarrow S^{\prime}$ be a morphism in $\operatorname{Def}_{k}$. Assume $S$ is of $K$-dimension $s, S^{\prime}$ if of $K$-dimension $s^{\prime}$ and $f$ is equidimensional of dimension $d=s-s^{\prime}$. A Function $\varphi$ in $C_{+}^{s}(S)$ is $f$-integrable if and only if $\varphi_{\mid S_{y}}\left(\frac{\left|\omega_{0}\right|_{S}}{\left|\omega_{0}\right|_{S^{\prime}}}\right)_{y}$ is integrable for every point $y$ in $S^{\prime}$ outside a definable subassignment of $K$-dimension $<s^{\prime}$. Then, for every point $y$ in $S^{\prime}$ outside a definable subassignment of $K$-dimension $<s^{\prime}$, we have

$$
i_{y}^{*}\left(f_{!}(\varphi)\right)=\int_{S_{y}} \varphi_{\mid S_{y}}\left(\frac{\left|\omega_{0}\right|_{S}}{\left|\omega_{0}\right|_{S^{\prime}}}\right)_{y} .
$$

Proof. - Let $\varphi$ be in $C_{+}^{s}(S)$. Assume $f=g \circ h$, with $g$ and $h$ satisfying the hypotheses of the proposition. Then, if the statement holds for $g$ and $h$, it also holds for $f$. Hence, using the embedding of $S$ into the graph of $f$, it is enough to prove the statement when $f$ is an isomorphism or when $f: S \subset S^{\prime}[m, n, r] \rightarrow S^{\prime}$ is induced by the projection. In the first case the statement follows from Theorem 12.1.1. For the second case one reduces similarly to proving the result when $m=0$, which is clear by 5.8.1, and when $(m, n, r)=(1,0,0)$. In this last case, by using a cell decomposition adapted to $\varphi$, one reduces to the case where $S$ is a cell and $\varphi=\left[\mathbf{1}_{S}\right]$. One also may assume $S$ is equal to its presentation. When $S$ is a 0-cell, 
the result follows from the case when $f$ is an isomorphism. When $S$ is a 1-cell, $\left(\frac{\left|\omega_{0}\right|_{S}}{\left|\omega_{0}\right|_{S^{\prime}}}\right)_{y}$ is nothing else than the restriction of the canonical volume form on $h[0,0,1]$ and the result follows from A7.

Proposition 15.4.1 should be compared with the following one, which should give a clear explanation of the difference between $f_{!}$and $\mu_{S^{\prime}}$.

15.4.2. Proposition. - Let $f: S \rightarrow S^{\prime}$ be a morphism in Def $_{k}$. Assume $S$ is of $K$-dimension $s, S^{\prime}$ if of $K$-dimension $s^{\prime}$ and that the fibers $S_{y}$ of $f$ are all of dimension $d=s-s^{\prime}$. Let $\varphi$ be a Function in $\mathrm{I}_{S^{\prime}} C_{+}^{d}\left(S \rightarrow S^{\prime}\right)$ or in $\mathrm{I}_{S^{\prime}} C^{d}\left(S \rightarrow S^{\prime}\right)$. Then, for every point $y$ in $S^{\prime}$, we have

$$
i_{y}^{*}\left(\mu_{S^{\prime}}(\varphi)\right)=\int_{S_{y}} \varphi_{\mid S_{y}}\left|\omega_{0}\right|_{S_{y}} .
$$

Proof. - It is enough to consider the positive case, which follows directly from Proposition 14.2.2.

15.4.3. Remark. - It is possible to generalize this section 15 to a relative setting, cf. remark 8.5.1. We will not give more details here.

\section{Comparison with the previous constructions of motivic integration}

16.1. Remarks about changing theories. - Let $T$ be a theory as in 2.7. Let $Z$ be a definable $T$-subassignment over $k$ (meaning that one allows coefficients from $k$ in the residue field sort and from $k((t))$ in the valued field sort). We may consider the subcategory $\operatorname{RDef}_{Z}\left(\mathcal{L}_{\mathrm{DP}, \mathrm{P}}, T\right)$ of $\operatorname{GDef}_{Z}\left(\mathcal{L}_{\mathrm{DP}, \mathrm{P}}, T\right)$, whose objects are definable $T$-subassignments $Y$ of $Z \times h_{\mathbb{A}_{k}^{n}}$, for some $n$, the morphism $Y \rightarrow Z$ being induced by projection on the $Z$ factor. One defines then similarly as in 5.1 the Grothendieck semiring and ring $S K_{0}\left(\operatorname{RDef}_{Z}\left(\mathcal{L}_{\text {DP }, \mathrm{P}}, T\right)\right)$ and $K_{0}\left(\operatorname{RDef}_{Z}\left(\mathcal{L}_{\text {DP }, \mathrm{P}}, T\right)\right)$, which we shall from now on write $S K_{0}\left(\operatorname{RDef}_{Z}\right)$ and $K_{0}\left(\operatorname{RDef}_{Z}\right)$ to make short. One also defines the semiring $\mathcal{P}_{+}\left(Z,\left(\mathcal{L}_{\mathrm{DP}, \mathrm{P}}, T\right)\right)$ and the ring $\mathcal{P}\left(Z,\left(\mathcal{L}_{\mathrm{DP}, \mathrm{P}}, T\right)\right)$ similarly as in 4.6 and also $\mathscr{C}_{+}\left(Z,\left(\mathcal{L}_{\mathrm{DP}, \mathrm{P}}, T\right)\right), \mathscr{C}\left(Z,\left(\mathcal{L}_{\mathrm{DP}, \mathrm{P}}, T\right)\right), C_{+}\left(Z,\left(\mathcal{L}_{\mathrm{DP}, \mathrm{P}}, T\right)\right), C\left(Z,\left(\mathcal{L}_{\mathrm{DP}, \mathrm{P}}, T\right)\right)$, $C_{+}\left(Z \rightarrow S,\left(\mathcal{L}_{\mathrm{DP}, \mathrm{P}}, T\right)\right)$ and $C\left(Z \rightarrow S,\left(\mathcal{L}_{\mathrm{DP}, \mathrm{P}}, T\right)\right)$ as in 5.3 and $\xi 6$. Here again, to make short we shall sometimes write $\mathcal{P}_{+}(Z)$ for $\mathcal{P}_{+}\left(Z,\left(\mathcal{L}_{\mathrm{DP}, \mathrm{P}}, T\right)\right)$, and so on. Everything we did in sections 5 to 15 extends mutatis mutandis to this more general framework.

Furthermore, all these constructions are functorial with respect to the theories in the following sense. Let $i: T_{1} \rightarrow T_{2}$ be an inclusion of theories and let $Z$ be a definable $T_{1}$-subassignment over $k$. Since $\operatorname{Field}_{k}\left(T_{2}\right)$ is a subcategory of Field $\operatorname{Si}_{k}\left(T_{1}\right)$, by restriction from Field ${ }_{k}\left(T_{1}\right)$ to Field $_{k}\left(T_{2}\right)$ we get a definable $T_{2}$-subassignment over $k$ we shall denote by $i_{*}(Z)$. In this way we get natural functors $i_{*}: \operatorname{GDef}_{k}\left(\mathcal{L}_{\mathrm{DP}, \mathrm{P}}, T_{1}\right) \rightarrow$ $\operatorname{GDef}_{k}\left(\mathcal{L}_{\mathrm{DP}, \mathrm{P}}, T_{2}\right)$ and $i_{*}: \operatorname{Def}_{k}\left(\mathcal{L}_{\mathrm{DP}, \mathrm{P}}, T_{1}\right) \rightarrow \operatorname{Def}_{k}\left(\mathcal{L}_{\mathrm{DP}, \mathrm{P}}, T_{2}\right)$. Note also that $i_{*}$ induces a functor $i_{*}: \operatorname{Def}_{Z}\left(\mathcal{L}_{\mathrm{DP}, \mathrm{P}}, T_{1}\right) \rightarrow \operatorname{Def}_{i_{*} Z}\left(\mathcal{L}_{\mathrm{DP}, \mathrm{P}}, T_{2}\right)$, hence a morphism $i_{*}: S K_{0}\left(\operatorname{RDef}_{Z}\right) \rightarrow S K_{0}\left(\operatorname{RDef}_{i_{*}}\right)$. Also, by restriction from $Z$ to $i_{*} Z$, one gets a morphism $i_{*}: \mathcal{P}_{+}(Z) \rightarrow \mathcal{P}_{+}\left(i_{*} Z\right)$ sending $\mathcal{P} 0_{+}(Z)$ on $\mathcal{P} 0_{+}\left(i_{*} Z\right)$, hence we have 
a natural morphism $i_{*}: \mathscr{C}_{+}(Z) \rightarrow \mathscr{C}_{+}\left(i_{*} Z\right)$, and similarly for $\mathscr{C}(Z), C_{+}(Z), C(Z)$, $C_{+}(Z \rightarrow S), C(Z \rightarrow S)$, etc.

The following statement, which follows directly from our constructions, is a typical example of what we mean by being functorial. Similar statements hold in the relative and global settings.

16.1.1. Proposition. - Let $i: T_{1} \rightarrow T_{2}$ be an inclusion of theories and let $S$ be in $\operatorname{Def}_{k}\left(\mathcal{L}_{\mathrm{DP}, \mathrm{P}}, T_{1}\right)$. Let $f: X \rightarrow Y$ be a morphism in $\operatorname{Def}_{k}\left(\mathcal{L}_{\mathrm{DP}, \mathrm{P}}, T_{1}\right)$. The morphism $i_{*}: C(X) \rightarrow C\left(i_{*} X\right)$ sends $S$-integrable Functions to $i_{*} S$-integrable Functions and

$$
i_{*} \circ f_{!}=\left(i_{*}(f)\right) ! \circ i_{*}
$$

For $S$ a subring of $k((t))$, if one restricts the coefficients in the valued field sort to $S$ and in the residue field sort to $k$, one can use the categories $\operatorname{Def}_{k}\left(\mathcal{L}_{\mathrm{DP}, \mathrm{P}}(S), T\right)$ and $\operatorname{GDef}_{k}\left(\mathcal{L}_{\mathrm{DP}, \mathrm{P}}(S), T\right)$ as defined in 2.7. For $Z$ in $\operatorname{Def}_{k}\left(\mathcal{L}_{\mathrm{DP}, \mathrm{P}}(S), T\right)$, one can then define correspondingly $\operatorname{RDef}_{Z}\left(\mathcal{L}_{\mathrm{DP}, \mathrm{P}}(S), T\right), S K_{0}\left(\operatorname{RDef}_{Z}\left(\mathcal{L}_{\mathrm{DP}, \mathrm{P}}(S), T\right)\right)$, $\mathcal{P}_{+}\left(Z,\left(\mathcal{L}_{\mathrm{DP}, \mathrm{P}}(S), T\right)\right), \mathscr{C}_{+}\left(Z,\left(\mathcal{L}_{\mathrm{DP}, \mathrm{P}}(S), T\right)\right), C_{+}\left(Z,\left(\mathcal{L}_{\mathrm{DP}, \mathrm{P}}(S), T\right)\right)$, and so on. In sections 16.3 and 16.4, we will take $S=k$ as coefficients in the valued field sort, in order to be able to compare with the previous constructions of motivic integration.

16.2. Restriction to $T_{\mathrm{acl}}$ : the geometric case. - Let us spell out the case when $T_{1}=T_{\emptyset}$ is the empty theory and $T_{2}$ is the theory $T_{\text {acl }}$ of algebraically closed fields containing $k$.

Of course, $\operatorname{Def}_{k}\left(\mathcal{L}_{\mathrm{DP}, \mathrm{P}}, T_{\emptyset}\right)=\operatorname{Def}_{k}$, so let us describe $\operatorname{Def}_{k}\left(\mathcal{L}_{\mathrm{DP}, \mathrm{P}}, T_{\text {acl }}\right)$.

By abuse of notation we shall still write $h[m, n, r]$ for $i_{*} h[m, n, r]$. By DenefPas quantifier elimination 2.1.1, Presburger quantifier quantifier elimination, and quantifier elimination for $T_{\mathrm{acl}}$ (= Chevalley constructibility), every object of $\operatorname{Def}_{k}\left(\mathcal{L}_{\mathrm{DP}, \mathrm{P}}, T_{\mathrm{acl}}\right)$ is defined by a $\mathcal{L}_{\mathrm{DP}, \mathrm{P}}$-formula without quantifiers. In particular, for $Z$ in $\operatorname{Def}_{k}\left(\mathcal{L}_{\mathrm{DP}, \mathrm{P}}, T_{\mathrm{acl}}\right)$, objects of $\operatorname{RDef}_{Z}$ can be seen as constructible sets (in the sense of algebraic geometry) parameterized by $Z$. For example, if $Z$ is a subassignment of $h[0, n, 0]$ defined by the vanishing of a familly of polynomials $f_{i}$, then $K_{0}\left(\operatorname{RDef}_{Z}\right)=K_{0}\left(\operatorname{Var}_{Z}\right)$, where we still write $Z$ for the affine algebraic variety defined by the vanishing of the polynomials $f_{i}$, and $\operatorname{Var}_{Z}$ denotes the category of algebraic varieties with a morphism to $Z$.

To have a neat description of $\mathscr{C}_{+}\left(Z,\left(\mathcal{L}_{\mathrm{DP}, \mathrm{P}}, T_{\mathrm{acl}}\right)\right)$, for $Z$ in $\operatorname{Def}_{k}\left(\mathcal{L}_{\mathrm{DP}, \mathrm{P}}, T_{\text {acl }}\right)$, it is enough to describe morphisms $Z \rightarrow h[0,0, r]$ and in fact to describe morphisms $h[m, n, r] \rightarrow h[0,0,1]$ in $\operatorname{Def}_{k}\left(\mathcal{L}_{\mathrm{DP}, \mathrm{P}}, T_{\mathrm{acl}}\right)$.

16.2.1. Proposition. - Let $f: h[m, n, r] \rightarrow h[0,0,1]$ be a morphism in $\operatorname{Def}_{k}\left(\mathcal{L}_{\mathrm{DP}, \mathrm{P}}, T_{\mathrm{acl}}\right)$. There exist polynomials $f_{1}, \ldots, f_{s}$ in $k((t))\left[x_{1}, \ldots, x_{m}\right]$, polynomials $g_{1}, \ldots, g_{s^{\prime}}, h_{1}, \ldots, h_{s^{\prime \prime}}$ in $k\left[t_{1}, \ldots, t_{n+s}\right]$, and a Presburger function $F: \mathbb{Z}^{N} \rightarrow \mathbb{Z}$, with $N=r+s+s^{\prime}+s^{\prime \prime}$, such that

$$
f(x, \xi, \alpha)=F\left(\operatorname{ord}_{0} f_{i}(x), \mathbf{1}_{g_{j}=0}\left(\xi, \overline{\mathrm{ac}} f_{i}(x)\right), \mathbf{1}_{h_{\ell} \neq 0}\left(\xi, \overline{\mathrm{ac}} f_{i}(x)\right), \alpha\right),
$$


where ord $\mathrm{d}_{0}$ is the map ord expanded by ord $\mathrm{o}_{0}(0)=0$, and where $\mathbf{1}_{g_{j}=0}$, resp. $\mathbf{1}_{h_{\ell} \neq 0}$, is the characteristic function of $g_{j}=0$, resp. of $h_{\ell} \neq 0$, on $h[0, n+s, 0]$ for each $j$ and $\ell$.

Proof. - Since, for $f_{i}$ polynomials in $k((t))\left[x_{1}, \ldots, x_{m}\right]$, conditions of the form $f_{i}=0$ or of the form $f_{i} \neq 0$ are equivalent to $\overline{\mathrm{ac}} f_{i}=0$ or $\overline{\mathrm{ac}} f_{i} \neq 0$, one may assume that the graph of $f$ is given by a formula where the Val-variables only occur in the forms $\overline{\mathrm{ac}} f_{i}$ and ord $f_{i}$ with $f_{i}$ polynomials in $k((t))\left[x_{1}, \ldots, x_{m}\right]$. Now the result follows from quantifier elimination.

16.3. Comparison with the original construction of motivic integration. - We restrict from now on the coefficients in the valued field sort to take values in $k$. If one considers the theory $T_{\text {acl }}$ of algebraically closed fields containing $k$, then $K_{0}\left(\operatorname{RDef}_{k}\left(\mathcal{L}_{\mathrm{DP}, \mathrm{P}}(k), T_{\mathrm{acl}}\right)\right)$ is nothing else but the ring $K_{0}\left(\operatorname{Var}_{k}\right)$ of [14], so we get a canonical morphism

$$
\gamma: S K_{0}\left(\mathrm{RDef}_{k}\right) \otimes_{\mathbb{N}[\mathbb{L}-1]} \mathbb{A}_{+} \longrightarrow K_{0}\left(\operatorname{Var}_{k}\right) \otimes_{\mathbb{Z}[\mathbb{L}]} \mathbb{A} .
$$

Here, $\mathbb{A}$ and $\mathbb{A}_{+}$are as defined as in 4.2 . Also, if we denote by $\widehat{\mathcal{M}}$ the completion of $K_{0}\left(\operatorname{Var}_{k}\right)\left[\mathbb{L}^{-1}\right]$ considered in [14], expanding the series $1-\mathbb{L}^{-i}$ yields a canonical morphism $\delta: K_{0}\left(\operatorname{Var}_{k}\right) \otimes_{\mathbb{Z}[\mathbb{L}]} \mathbb{A} \rightarrow \widehat{\mathcal{M}}$.

Let $X$ be an algebraic variety of dimension $d$ over $k$. Set $\mathcal{X}_{0}:=X \otimes_{\operatorname{Spec} k} \operatorname{Spec} k \llbracket t \rrbracket$ and $\mathcal{X}:=\mathcal{X}_{0} \otimes_{\text {Spec } k \llbracket t \rrbracket}$ Spec $k((t))$. Consider a definable subassignment $W$ of $h_{\mathcal{X}}$ in the language $\mathcal{L}_{\mathrm{DP}, \mathrm{P}}(k)$. If $\mathcal{X}$ is affine and embedded as a closed subscheme in $\mathbb{A}_{k((t))}^{n}$, such that the embedding is defined over $k \llbracket t \rrbracket$, we call $W$ small if $W \subset Y$ with $Y$ the subassignment of $h[n, 0,0]$ given by ord $x_{i} \geq 0$ where $x_{i}$ are coordinates on $h[n, 0,0]$. In general we call $W$ small if there is an open affine cover $U_{i}$ of $\mathcal{X}$, defined over $k \llbracket t \rrbracket$, such that the intersections $W \cap h_{U_{i}}$ are small. Assume that $W$ is small. Then, formulas defining $W$ (in affine open charts defined over $k$ ) define a semi-algebraic subset of the arc space $\mathcal{L}(X)$ (in the corresponding chart, with the notations of [14]), by quantifier elimination for algebraically closed fields and for $\mathbb{Z}$ in the Presburger language. In this way one may assign canonically to every small $W$ a semi-algebraic subset $\tilde{W}$ of $\mathcal{L}(X)$. Similarly, every $\mathbb{Z}$-valued function $\alpha$ on $W$ which is definable in the language $\mathcal{L}_{\mathrm{DP}, \mathrm{P}}(k)$ gives rise to a semi-algebraic function $\tilde{\alpha}$ on $\tilde{W}$.

16.3.1. Theorem. - Under the previous assumptions, if $\left|\omega_{0}\right|$ denotes the canonical volume form on $h_{\mathcal{X}}$ defined in 8.6. for any bounded below $\mathbb{Z}$-valued definable function $\alpha$ on $W, \mathbf{1}_{W} \mathbb{L}^{-\alpha}\left|\omega_{0}\right|$ is integrable on $h_{\mathcal{X}}$ and we have

$$
(\delta \circ \gamma)\left(\int_{h_{\mathcal{X}}} \mathbf{1}_{W} \mathbb{L}^{-\alpha}\left|\omega_{0}\right|\right)=\int_{\tilde{W}} \mathbb{L}^{-\tilde{\alpha}} d \mu^{\prime},
$$

with $\mu^{\prime}$ denoting the motivic measure defined in [14].

16.3.2. Remark. - The above result shows that for semi-algebraic sets and functions the motivic volume of [14] already exists at the level of $K_{0}\left(\operatorname{Var}_{k}\right) \otimes_{\mathbb{Z}[\mathbb{L}]} \mathbb{A}$, and even at the level of $S K_{0}\left(\operatorname{RDef}_{k}\right) \otimes_{\mathbb{N}[\mathbb{L}-1]} \mathbb{A}_{+}$, that is, before any completion process. 
Proof. - The statement concerning integrability follows directly from Proposition 12.2.2. Similarly as what is performed in the proof of Theorem $5.1^{\prime}$ in [14], we may reduce to the case where $X$ is affine and, using resolution of singularities and the change of variable formula Theorem 15.3.1, we may assume that all the functions $f_{i}$ and $h$ occurring in the semi-algebraic description [14 (2.1) (i)-(iii) of $\tilde{W}$ and $\tilde{\alpha}$ are monomials. The integrals we have to compare are then products of similar integrals in one variable which are equal by direct computation.

16.4. Comparison with arithmetic integration. - Recall that we restrict the coefficients in the valued field sort to $k$. Now consider the theory PFF of pseudofinite fields containing $k$. Then $K_{0}\left(\operatorname{RDef}_{k}\left(\mathcal{L}_{\mathrm{DP}, \mathrm{P}}(k), \mathrm{PFF}\right)\right)$ is nothing else but the ring denoted by $K_{0}\left(\mathrm{PFF}_{k}\right)$ in [16] and [17. In [15], arithmetic integration was defined as taking values in the completion $\hat{K}_{0}^{v}\left(\operatorname{Mot}_{k, \overline{\mathbb{Q}}}\right)_{\mathbb{Q}}$ of a ring $K_{0}^{v}\left(\operatorname{Mot}_{k, \overline{\mathbb{Q}}}\right)_{\mathbb{Q}}$. It was somewhat later remarked in [16] and [17] that one can consider a smaller ring denoted by $K_{0}^{\operatorname{mot}}\left(\operatorname{Var}_{k}\right) \otimes \mathbb{Q}$, whose definition we shall now recall. For $k$ a field of characteristic zero, there exists by Gillet and Soulé [21], Guillen and NavarroAznar [22], a unique ring morphism $K_{0}\left(\operatorname{Var}_{k}\right) \rightarrow K_{0}\left(\mathrm{CHMot}_{k}\right)$, which assigns to the class of a smooth projective variety $X$ over $k$ the class of its Chow motive, where $K_{0}\left(\mathrm{CHMot}_{k}\right)$ denotes the Grothendieck ring of the category of Chow motives over $k$ (with rational coefficients). By definition $K_{0}^{\operatorname{mot}}\left(\operatorname{Var}_{k}\right)$ is the image of $K_{0}\left(\operatorname{Var}_{k}\right)$ in $K_{0}\left(\mathrm{CHMot}_{k}\right)$ under this morphism. [Note that the definition of $K_{0}^{\operatorname{mot}}\left(\operatorname{Var}_{k}\right)$ given in [16] is not clearly equivalent and should be replaced by the one given above.] In [16] and [17, building on the work in [15], a canonical morphism

$$
\chi_{c}: K_{0}\left(\mathrm{PFF}_{k}\right) \longrightarrow K_{0}^{\mathrm{mot}}\left(\operatorname{Var}_{k}\right) \otimes \mathbb{Q}
$$

was constructed. Recently, J. Nicaise has extended that construction to the relative setting [29].

The arithmetic motivic measure takes values in a certain completion $\hat{K}_{0}^{\text {mot }}\left(\operatorname{Var}_{k}\right) \otimes$ $\mathbb{Q}$ of the localization of $K_{0}^{\operatorname{mot}}\left(\operatorname{Var}_{k}\right) \otimes \mathbb{Q}$ with respect to the class of (the image of) the affine line. We have natural morphisms $\tilde{\gamma}: S K_{0}\left(\operatorname{RDef}_{k}\right) \otimes \mathbb{N}[\mathbb{L}-1] \mathbb{A}_{+} \rightarrow$ $K_{0}\left(\mathrm{PFF}_{k}\right) \otimes_{\mathbb{Z}[\mathbb{L}]} \mathbb{A}$. The morphism $\chi_{c}$ induces, after taking series expansions of $\left(1-\mathbb{L}^{-i}\right)^{-1}$, a canonical morphism $\tilde{\delta}: K_{0}\left(\mathrm{PFF}_{k}\right) \otimes_{\mathbb{Z}[\mathbb{L}]} \mathbb{A} \rightarrow \hat{K}_{0}^{\operatorname{mot}}\left(\operatorname{Var}_{k}\right) \otimes \mathbb{Q}$.

Let $X$ be an algebraic variety of dimension $d$ over $k$. Set $\mathcal{X} 0:=X \otimes_{\text {Spec } k} \operatorname{Spec} k \llbracket t \rrbracket$ and $\mathcal{X}:=\mathcal{X} 0 \otimes_{\text {Spec } k \llbracket t \rrbracket}$ Spec $k((t))$. Consider a definable subassignment $W$ of $h_{\mathcal{X}}$ in the language $\mathcal{L}_{\mathrm{DP}, \mathrm{P}}(k)$ which is small in the sense of 16.3. Clearly the formulas defining $W$ (in affine open charts defined over $k$ ) define a definable subassignment $\tilde{W}$ of $h_{\mathcal{L}(X)}$, with the notations of [15], by quantifier elimination for $\mathbb{Z}$ in the Presburger language.

16.4.1. Theorem. - Under the previous assumptions, if $\left|\omega_{0}\right|$ denotes the canonical volume form on $h_{\mathcal{X}}$ defined in 8.6, $\mathbf{1}_{W}\left|\omega_{0}\right|$ is integrable on $h_{\mathcal{X}}$ and we have

$$
(\tilde{\delta} \circ \tilde{\gamma})\left(\int_{h_{\mathcal{X}}} \mathbf{1}_{W}\left|\omega_{0}\right|\right)=\nu(\tilde{W})
$$


with $\nu$ denoting the arithmetic motivic measure defined in [15].

Proof. - Similar to the proof of Theorem 16.3.1.

16.4.2. Remark. - By Theorem 16.4.1 and by specialization properties of arithmetic motivic integrals to $p$-adic integrals for $p$ big enough [17], one sees that the present formalism of motivic integration is suited to interpolate $p$-adic integrals for $p$ big enough. For more detailed results than what follows from 16.4.1 and [17] and for a link with $\mathbb{F}_{q}((t))$-integrals, we refer to $[\mathbf{8}$.

16.4.3. Remark. - A (partial) comparison with the construction of motivic integration for formal schemes, as developed by J. Sebag [33], can also be made.

\section{References}

[1] N. Bourbaki, Variétés différentielles et analytiques. Fascicule de résultats, Hermann 1967.

[2] N. Bourbaki, Topologie générale, Chapitres 5 à 10, Hermann 1974.

[3] Y. F. Çelikler Dimension theory and parameterized normalization for D-semianalytic sets over non-Archimedean fields, J. Symbolic Logic, 70 (2005), 593-618.

[4] R. Cluckers, Presburger sets and p-minimal fields, J. Symbolic Logic, 68 (2003), 153162.

[5] R. Cluckers, L. Lipshitz, Z. Robinson, Analytic cell decomposition and analytic motivic integration, Ann. Sci. École Norm. Sup., 39 (2006), 535-568.

[6] R. Cluckers, F. Loeser, Fonctions constructibles et intégration motivique I, C. R. Math. Acad. Sci. Paris 339 (2004), 411-416.

[7] R. Cluckers, F. Loeser, Fonctions constructibles et intégration motivique II, C. R. Math. Acad. Sci. Paris 339 (2004), 487-492.

[8] R. Cluckers, F. Loeser, Ax-Kochen-Eršov Theorems for p-adic integrals and motivic integration, in Geometric methods in algebra and number theory, edited by F. Bogomolov and Y. Tschinkel, Progress in Mathematics 235, 109-137 (2005), Birkhäuser.

[9] R. Cluckers, F. Loeser, Fonctions constructibles exponentielles, transformation de Fourier motivique et principe de transfert, C. R. Acad. Sci. Paris Sér. I Math. 341 (2005), 741-746.

[10] R. Cluckers, F. Loeser, Constructible exponential functions, motivic Fourier transform and transfer principle, math.AG/0512022, to appear in Ann. of Math.

[11] J. Denef, The rationality of the Poincaré series associated to the p-adic points on a variety, Invent. Math., 77 (1984), 1-23.

[12] J. Denef, On the evaluation of certain p-adic integrals, Séminaire de théorie des nombres, Paris 1983-84, 25-47, Progr. Math., 59, Birkhäuser Boston, Boston, MA, 1985

[13] J. Denef, L. van den Dries, p-adic and real subanalytic sets, Ann. of Math., 128, (1988), 79-138.

[14] J. Denef, F. Loeser, Germs of arcs on singular algebraic varieties and motivic integration, Invent. Math. 135 (1999), 201-232.

[15] J. Denef, F. Loeser, Definable sets, motives and p-adic integrals, J. Amer. Math. Soc., 14 (2001), 429-469. 
[16] J. Denef, F. Loeser, Motivic integration and the Grothendieck group of pseudo-finite fields, Proceedings of the International Congress of Mathematicians, Vol. II (Beijing, 2002), 2002, 13-23, Higher Ed. Press, Beijing.

[17] J. Denef, F. Loeser, On some rational generating series occurring in arithmetic geometry, in Geometric aspects of Dwork theory. Vol. I, 509-526, Walter de Gruyter GmbH \& Co. KG, Berlin, 2004.

[18] L. van den Dries, Dimension of definable sets, algebraic boundedness and Henselian fields, Ann. Pure Appl. Logic, 45 (1989), 189-209.

[19] L. van den Dries, Analytic Ax-Kochen-Ersov theorems, Proceedings of the International Conference on Algebra, Part 3 (Novosibirsk, 1989), 379-398, Contemp. Math., 131, Part 3, Amer. Math. Soc., Providence, RI, 1992.

[20] L. van den Dries, Tame topology and o-minimal structures, London Mathematical Society Lecture Note Series 248, Cambridge Univ. Press (1998).

[21] H. Gillet, C. Soulé, Descent, motives and K-theory, J. Reine Angew. Math. 478 (1996), 127-176.

[22] F. Guillén, V. Navarro Aznar, Un critère d'extension d'un foncteur défini sur les schémas lisses, Inst. Hautes Études Sci. Publ. Math. 95 (2002), 1-91.

[23] R. Hartshorne, Algebraic geometry, Graduate Texts in Mathematics 52, SpringerVerlag, New York-Heidelberg, 1977.

[24] M. Kontsevich, Lecture at Orsay (December 7, 1995).

[25] E. Hrushovski, D. Kazhdan, Integration in valued fields, in Algebraic geometry and number theory, Progress in Mathematics 253, 261-405 (2006), Birkhäuser.

[26] J. Igusa, An introduction to the theory of local zeta functions, AMS/IP Studies in Advanced Mathematics, 14. International Press, Cambridge, MA, 2000.

[27] R. MacPherson, Chern classes for singular algebraic varieties, Ann. of Math. 100 (1974), 423-432.

[28] D. Marker, Model theory: An introduction, Graduate Texts in Mathematics 217, Springer-Verlag, New York, 2002.

[29] J. Nicaise, Relative motives and the theory of pseudo-finite fields, Int. Math. Res. Pap. IMRP 1 (2007), 69 pp.

[30] J. Pas, Uniform p-adic cell decomposition and local zeta functions, J. Reine Angew. Math., 399 (1989), 137-172.

[31] M. Presburger, Uber die Vollständigkeit eines gewissen Systems des Arithmetik ..., Comptes-rendus du I Congrès des Mathématiciens des Pays Slaves, Warsaw (1929), 92101.

[32] P. Schapira, Operations on constructible functions, J. Pure Appl. Algebra, 72 (1991), 83-93.

[33] J. Sebag, Intégration motivique sur les schémas formels, Bull. Soc. Math. France, 132 (2004), 1-54.

[34] J. Sebag, Rationalité des séries de Poincaré et des fonctions zêta motiviques, Manuscripta Math., 115 (2004), 125-162.

[35] J.-P. Serre, Quelques applications du théorème de densité de Chebotarev, Inst. Hautes Études Sci. Publ. Math., 54 (1981), 323-401.

[36] J.-P. Serre, Lie algebras and Lie groups, 1964 lectures given at Harvard University, Mathematics Lecture Notes Series (Benjamin, New York). 
[37] O. Viro, Some integral calculus based on Euler characteristic, Lecture Notes in Math, Springer-Verlag, 1346 (1988), 127-138.

Raf Cluckers, Katholieke Universiteit Leuven, Departement wiskunde, Celestijnenlaan 200B, B-3001 Leuven, Belgium. Current address: École Normale Supérieure, Département de mathématiques et applications, 45 rue d'Ulm, 75230 Paris Cedex 05, France E-mail : cluckers@ens.fr • Url : www.dma.ens.fr/ cluckers/

François Loeser, École Normale Supérieure, Département de mathématiques et applications, 45 rue d'Ulm, 75230 Paris Cedex 05, France (UMR 8553 du CNRS)

E-mail : Francois.Loeser@ens.fr • Url : www.dma.ens.fr/ loeser/ 